\title{
Phenolic Glycosides from the Roots of Ficus hirta Vahl. and Their Antineuroinflammatory Activities
}

\author{
Xiansheng Ye, ${ }^{\dagger, \| l}$ Wenjing Tian, ${ }^{\dagger, \| l}$ Guanghui Wang, ${ }^{\dagger, \| l}$ Xian Zhang, ${ }^{\ddagger}$ Mi \\ Zhou, ${ }^{\dagger}$ Dequan Zeng, ${ }^{\dagger}$ Xiangzhong Liu, ${ }^{\dagger}$ Xinsheng Yao, ${ }^{*}, \dagger, \S$ Yunwu \\ Zhang, $*,+$ and Haifeng Chen*,†
}

†Fujian Provincial Key Laboratory of Innovative Drug Target, School of Pharmaceutical Sciences, Xiamen University, Xiamen 361102, People's Republic of China

${ }^{\ddagger}$ Fujian Provincial Key Laboratory of Neurodegenerative Disease and Aging Research, Institute of Neuroscience, School of Medicine, Xiamen University, Xiamen 361102, People's Republic of China

\$nstitute of Traditional Chinese Medicine \& Natural Products, Jinan University, Guangzhou 510632, People's Republic of China

Corresponding Authors

*E-mail (H. Chen): haifeng@xmu.edu.cn.

*E-mail (Y. Zhang): yunzhang@xmu.edu.cn.

*E-mail (X. Yao): tyaoxs@jnu.edu.cn. 


\section{Supporting information}

\begin{tabular}{|c|c|c|}
\hline No. & Contents & Page \\
\hline 1 & Figure S1. ${ }^{1} \mathrm{H}-\mathrm{NMR}$ Spectrum of Compound 1 in Methanol- $d_{4}$ & 4 \\
\hline 2 & Figure S2. ${ }^{13}$ C-NMR Spectrum of Compound 1 in Methanol- $d_{4}$ & 4 \\
\hline 3 & Figure S3. ${ }^{1} \mathrm{H}-{ }^{1} \mathrm{H}$ COSY Spectrum of Compound $\mathbf{1}$ in Methanol- $d_{4}$ & 5 \\
\hline 4 & Figure S4. HSQC Spectrum of Compound $\mathbf{1}$ in Methanol- $d_{4}$ & 5 \\
\hline 5 & Figure S5. HMBC Spectrum of Compound 1 in Methanol- $d_{4}$ & 6 \\
\hline 6 & Figure S6. UV Spectrum of Compound 1 & 6 \\
\hline 7 & Figure S7. HRESIMS Spectrum of Compound 1 & 7 \\
\hline 8 & Figure S8. IR Spectrum of Compound 1 & 7 \\
\hline 9 & Figure S9. ${ }^{1} \mathrm{H}-\mathrm{NMR}$ Spectrum of Compound 2 in Methanol- $d_{4}$ & 8 \\
\hline 10 & Figure S10. ${ }^{13} \mathrm{C}-\mathrm{NMR}$ Spectrum of Compound 2 in Methanol- $d_{4}$ & 8 \\
\hline 11 & Figure S11. ${ }^{1} \mathrm{H}-{ }^{1} \mathrm{H}$ COSY Spectrum of Compound 2 in Methanol- $d_{4}$ & 9 \\
\hline 12 & Figure S12. HSQC Spectrum of Compound 2 in Methanol- $d_{4}$ & 9 \\
\hline 13 & Figure S13. HMBC Spectrum of Compound 2 in Methanol- $d_{4}$ & 10 \\
\hline 14 & Figure S14. UV Spectrum of Compound 2 & 10 \\
\hline 15 & Figure S15. HRESIMS Spectrum of Compound 2 & 11 \\
\hline 16 & Figure S16. IR Spectrum of Compound 2 & 11 \\
\hline 17 & Figure S17. ${ }^{1} \mathrm{H}-\mathrm{NMR}$ Spectrum of Compound 3 in Methanol- $d_{4}$ & 12 \\
\hline 18 & Figure S18. ${ }^{13}$ C-NMR Spectrum of Compound 3 in Methanol- $d_{4}$ & 12 \\
\hline 19 & Figure S19. ${ }^{1} \mathrm{H}-{ }^{1} \mathrm{H}$ COSY Spectrum of Compound 3 in Methanol- $d_{4}$ & 13 \\
\hline 20 & Figure S20. HSQC Spectrum of Compound $\mathbf{3}$ in Methanol- $d_{4}$ & 13 \\
\hline 21 & Figure S21. HMBC Spectrum of Compound 3 in Methanol- $d_{4}$ & 14 \\
\hline 22 & Figure S22. UV Spectrum of Compound 3 & 14 \\
\hline 23 & Figure S23. HRESIMS Spectrum of Compound 3 & 15 \\
\hline 24 & Figure S24. IR Spectrum of Compound 3 & 15 \\
\hline 25 & Figure S25. ${ }^{1} \mathrm{H}-\mathrm{NMR}$ Spectrum of Compound 4 in Methanol- $d_{4}$ & 16 \\
\hline 26 & Figure S26. ${ }^{13} \mathrm{C}-\mathrm{NMR}$ Spectrum of Compound 4 in Methanol- $d_{4}$ & 16 \\
\hline 27 & Figure S27. ${ }^{1} \mathrm{H}^{-1} \mathrm{H}$ COSY Spectrum of Compound 4 in Methanol- $d_{4}$ & 17 \\
\hline 28 & Figure S28. HSQC Spectrum of Compound $\mathbf{4}$ in Methanol- $d_{4}$ & 17 \\
\hline 29 & Figure S29. HMBC Spectrum of Compound 4 in Methanol- $d_{4}$ & 18 \\
\hline
\end{tabular}


Figure S33. ${ }^{1} \mathrm{H}-\mathrm{NMR}$ Spectrum of Compound $\mathbf{5}$ in Methanol- $d_{4} \quad 20$

Figure S34. ${ }^{13} \mathrm{C}$-NMR Spectrum of Compound $\mathbf{5}$ in Methanol- $d_{4} \quad 20$

Figure S35. ${ }^{1} \mathrm{H}-{ }^{1} \mathrm{H}$ COSY Spectrum of Compound 5 in Methanol- $d_{4} \quad 21$

Figure S36. HSQC Spectrum of Compound 5 in Methanol- $d_{4}$

Figure S37. HMBC Spectrum of Compound 5 in Methanol- $d_{4}$

Figure S38. UV Spectrum of Compound 5

Figure S39. HRESIMS Spectrum of Compound 5

Figure S40. IR Spectrum of Compound 5

Figure S41. ${ }^{1} \mathrm{H}-\mathrm{NMR}$ Spectrum of Compound 6 in Methanol- $d_{4} \quad 24$

Figure S42. ${ }^{13} \mathrm{C}$-NMR Spectrum of Compound 6 in Methanol- $d_{4}$

Figure S43. ${ }^{1} \mathrm{H}-{ }^{1} \mathrm{H}$ COSY Spectrum of Compound 6 in Methanol- $d_{4} \quad 25$

Figure S44. HSQC Spectrum of Compound 6 in Methanol- $d_{4}$

Figure S45. HMBC Spectrum of Compound 6 in Methanol- $d_{4} \quad 26$

Figure S46. UV Spectrum of Compound 6

Figure S47. HRESIMS Spectrum of Compound $6 \quad 27$

Figure S48. IR Spectrum of Compound 6

Figure S49. ${ }^{1} \mathrm{H}-\mathrm{NMR}$ Spectrum of Compound 7 in Methanol- $d_{4} \quad 28$

Figure S50. ${ }^{13} \mathrm{C}-\mathrm{NMR}$ Spectrum of Compound 7 in Methanol- $d_{4} \quad 28$

Figure S51. ${ }^{1} \mathrm{H}-{ }^{1} \mathrm{H}$ COSY Spectrum of Compound 7 in Methanol- $d_{4} \quad 29$

Figure S52. HSQC Spectrum of Compound 7 in Methanol- $d_{4}$

Figure S53. HMBC Spectrum of Compound 7 in Methanol- $d_{4}$

Figure S54. UV Spectrum of Compound $7 \quad 30$

Figure S55. HRESIMS Spectrum of Compound $7 \quad 31$

Figure S56. IR Spectrum of Compound 7

Figure S57. HPLC analysis for derivatives of D-glucose, L-glucose, 32

D-apiose and hydrolysates of Compound 1

Figure S58. HPLC analysis for derivatives of D-glucose, L-glucose,

D-rhamnose, L-rhamnose and hydrolysates of Compound 6

Figure S59. The origin Western blot picture of the p-Akt and p-ERK 70

Figure S60. The origin Western blot picture of the p-p65, p-Akt, p-ERK 70 and $\mathrm{p}-\mathrm{JNK}$ 


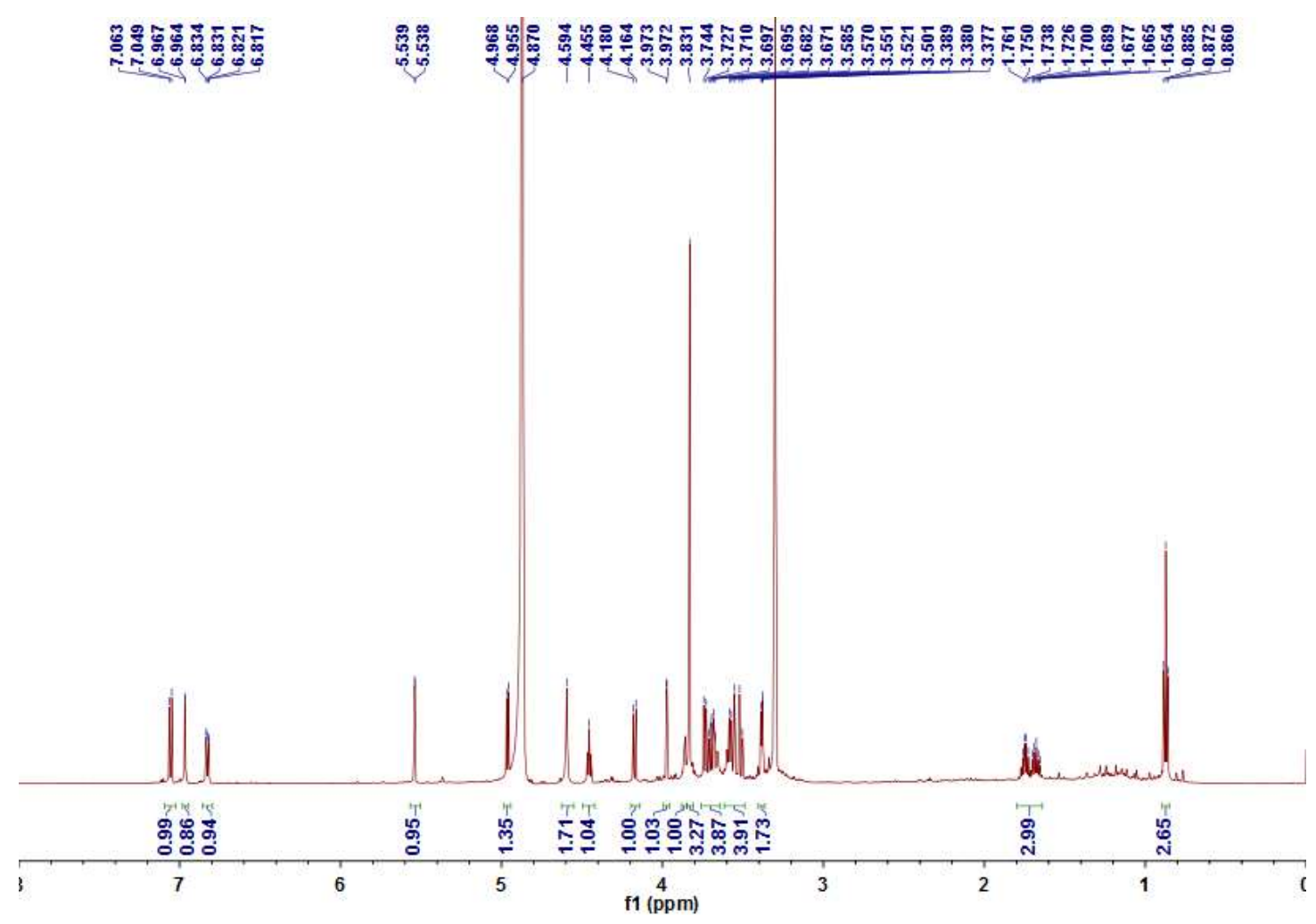

Figure S1. ${ }^{1}$ H-NMR Spectrum of Compound 1 in Methanol- $d_{4}$

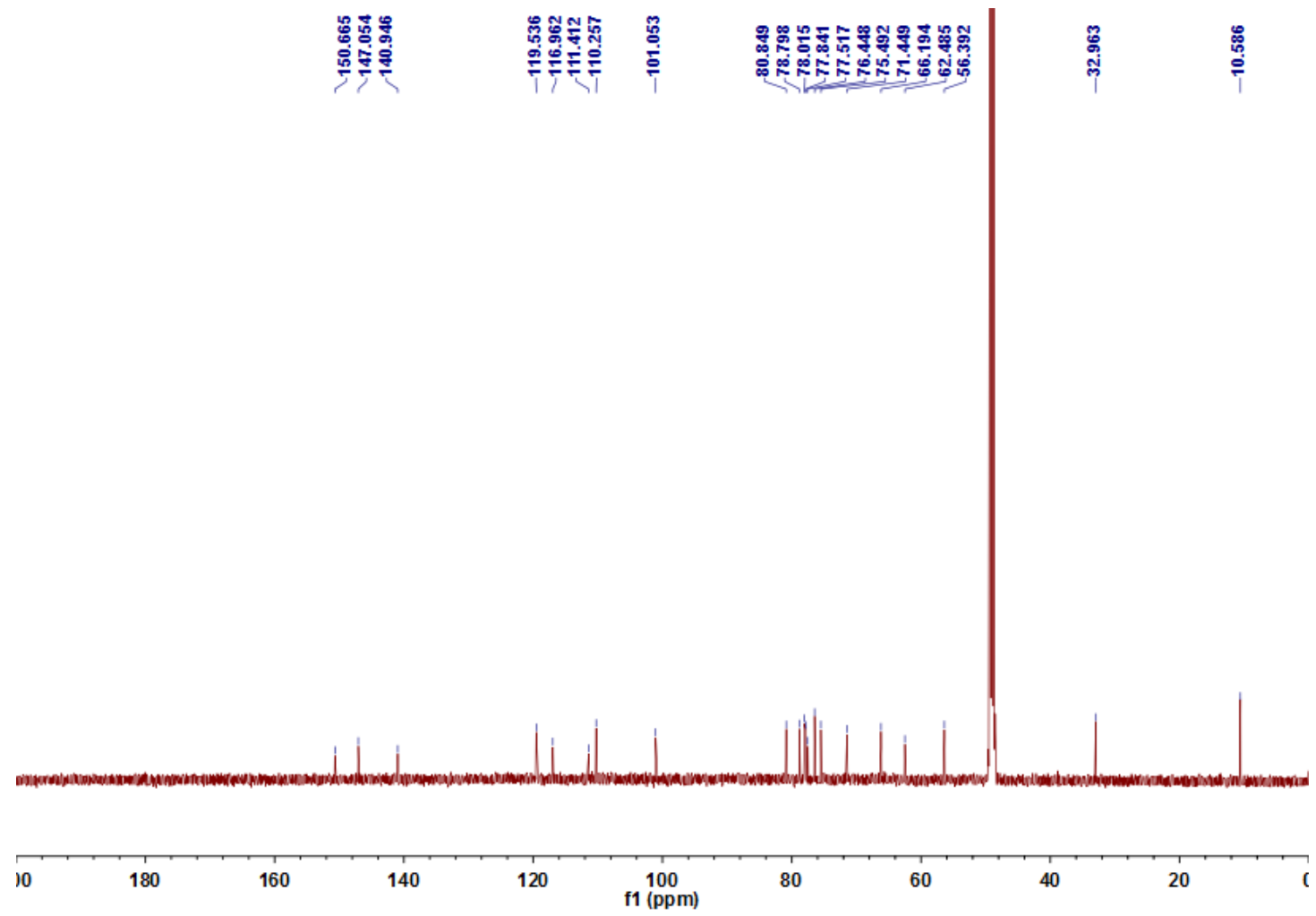

Figure S2. ${ }^{13}$ C-NMR Spectrum of Compound 1 in Methanol- $d_{4}$ 


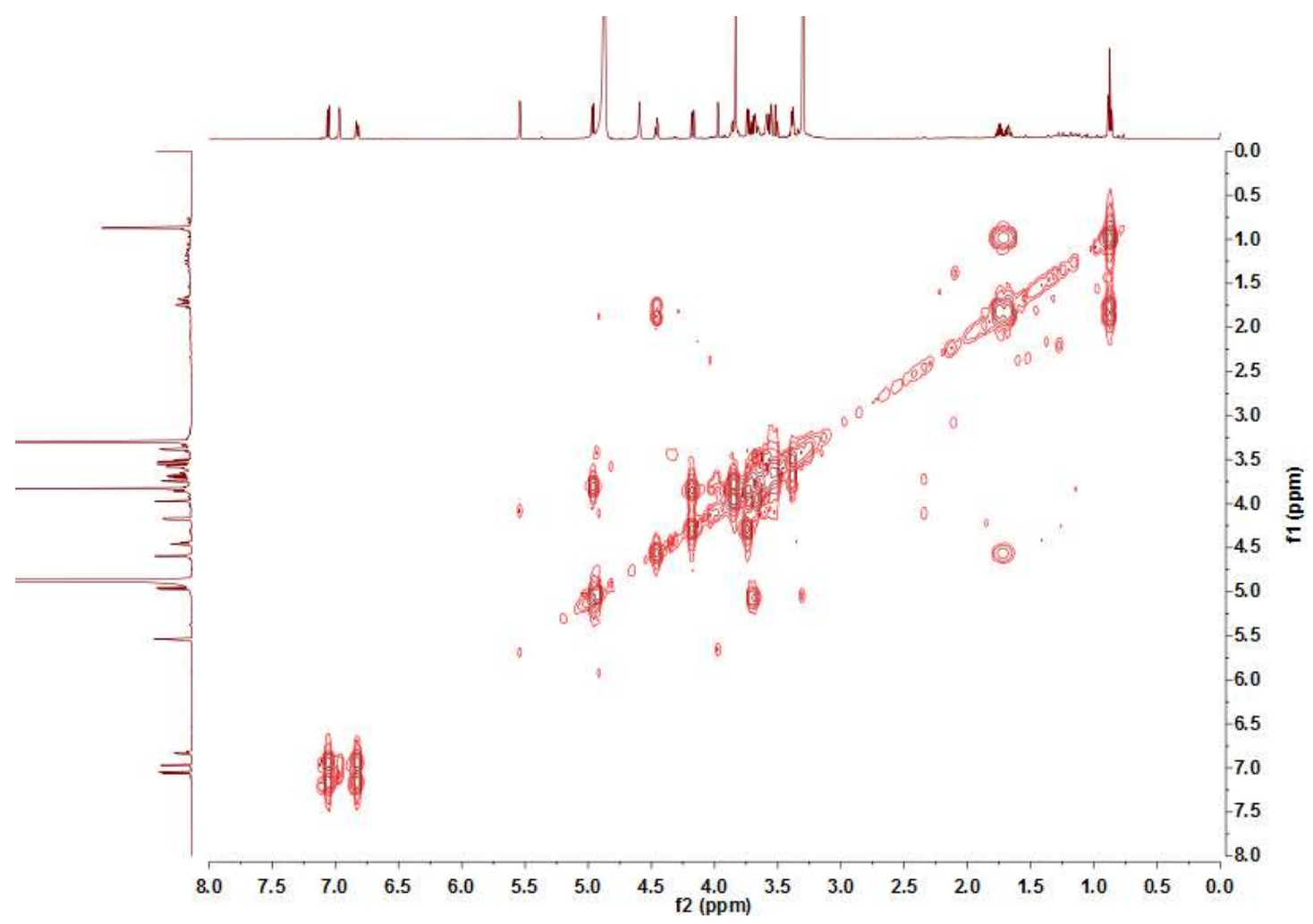

Figure S3. ${ }^{1} \mathrm{H}-{ }^{1} \mathrm{H}$ COSY Spectrum of Compound $\mathbf{1}$ in Methanol- $d_{4}$

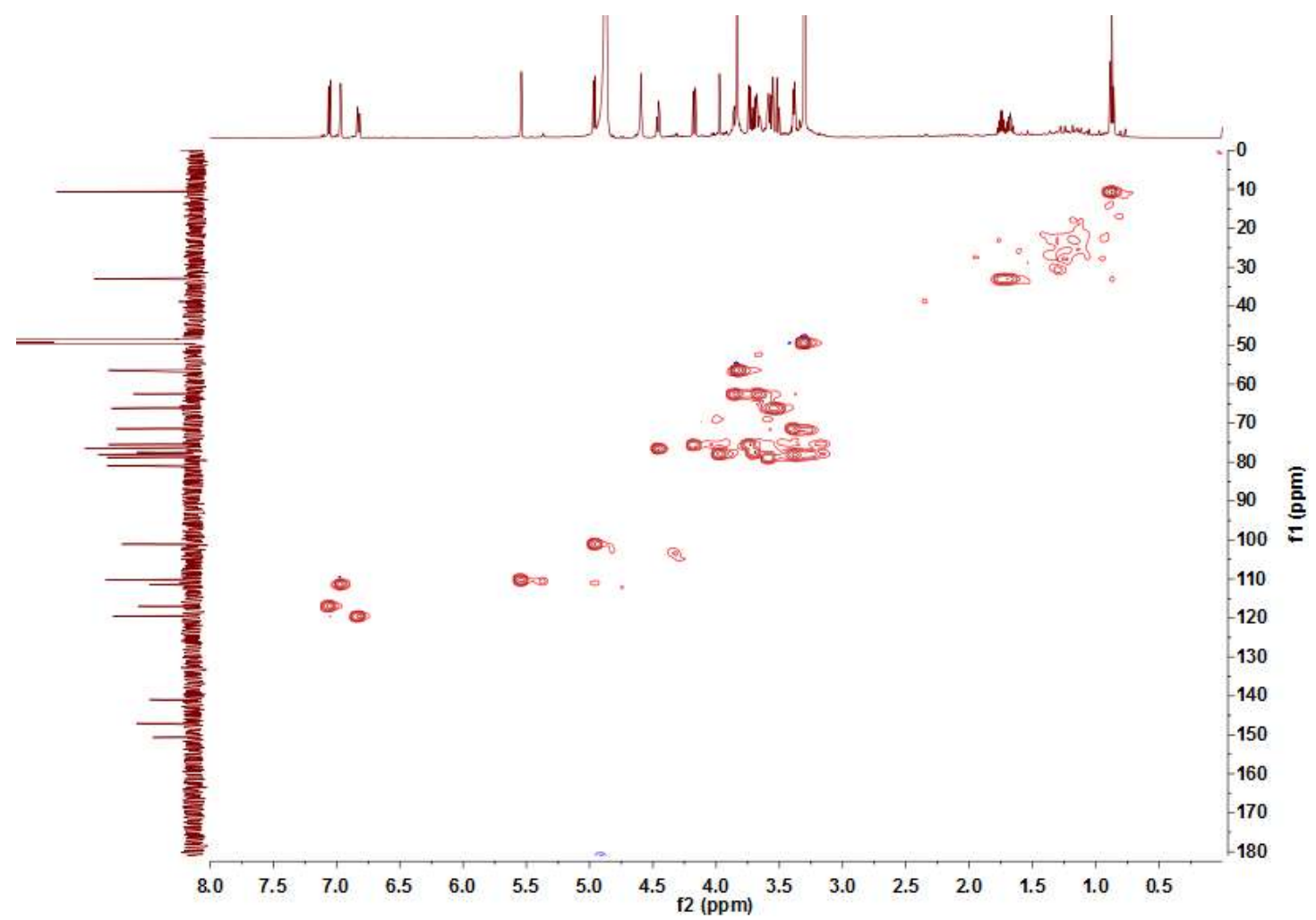

Figure S4. HSQC Spectrum of Compound 1 in Methanol- $d_{4}$ 


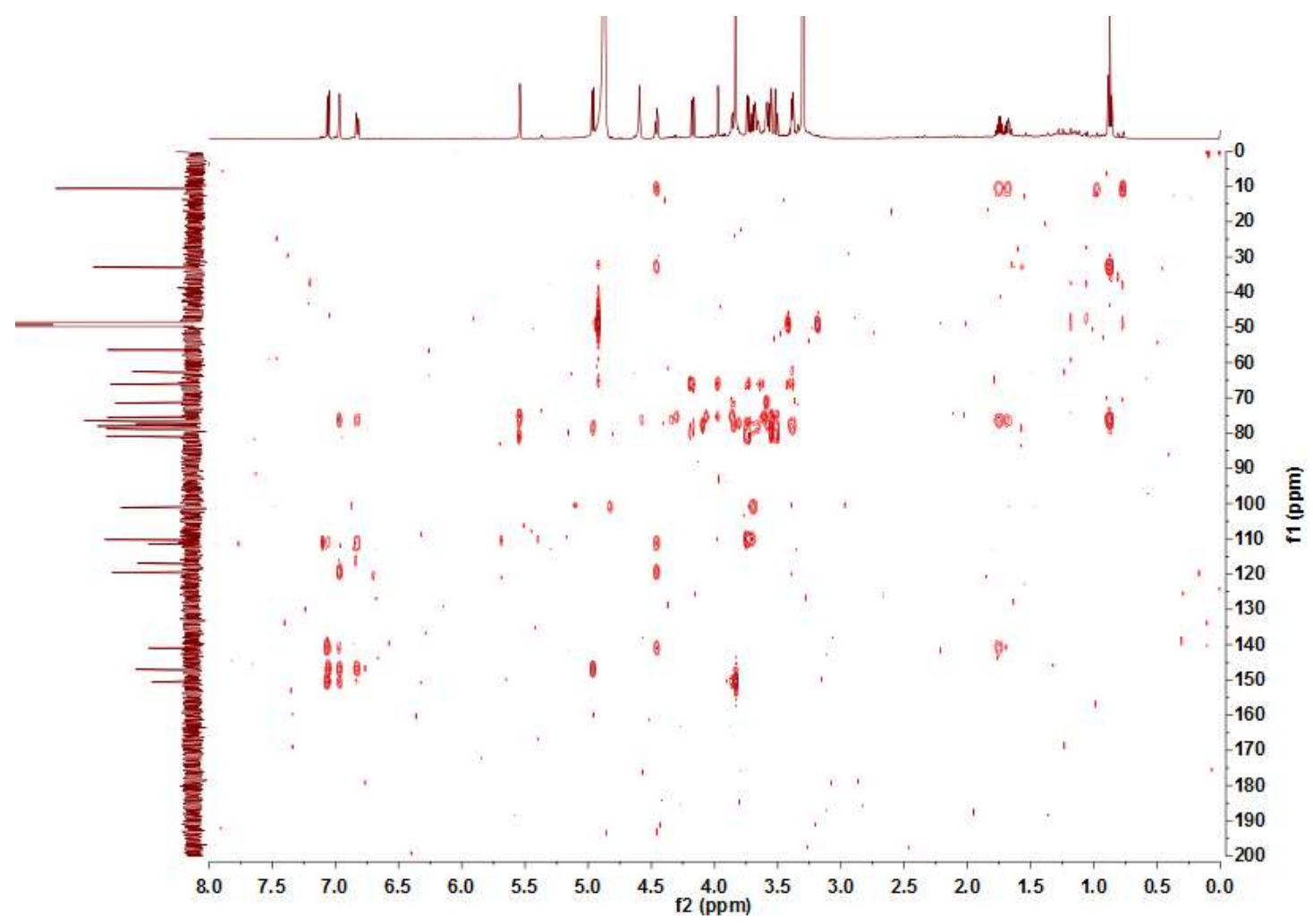

Figure S5. HMBC Spectrum of Compound 1 in Methanol- $d_{4}$

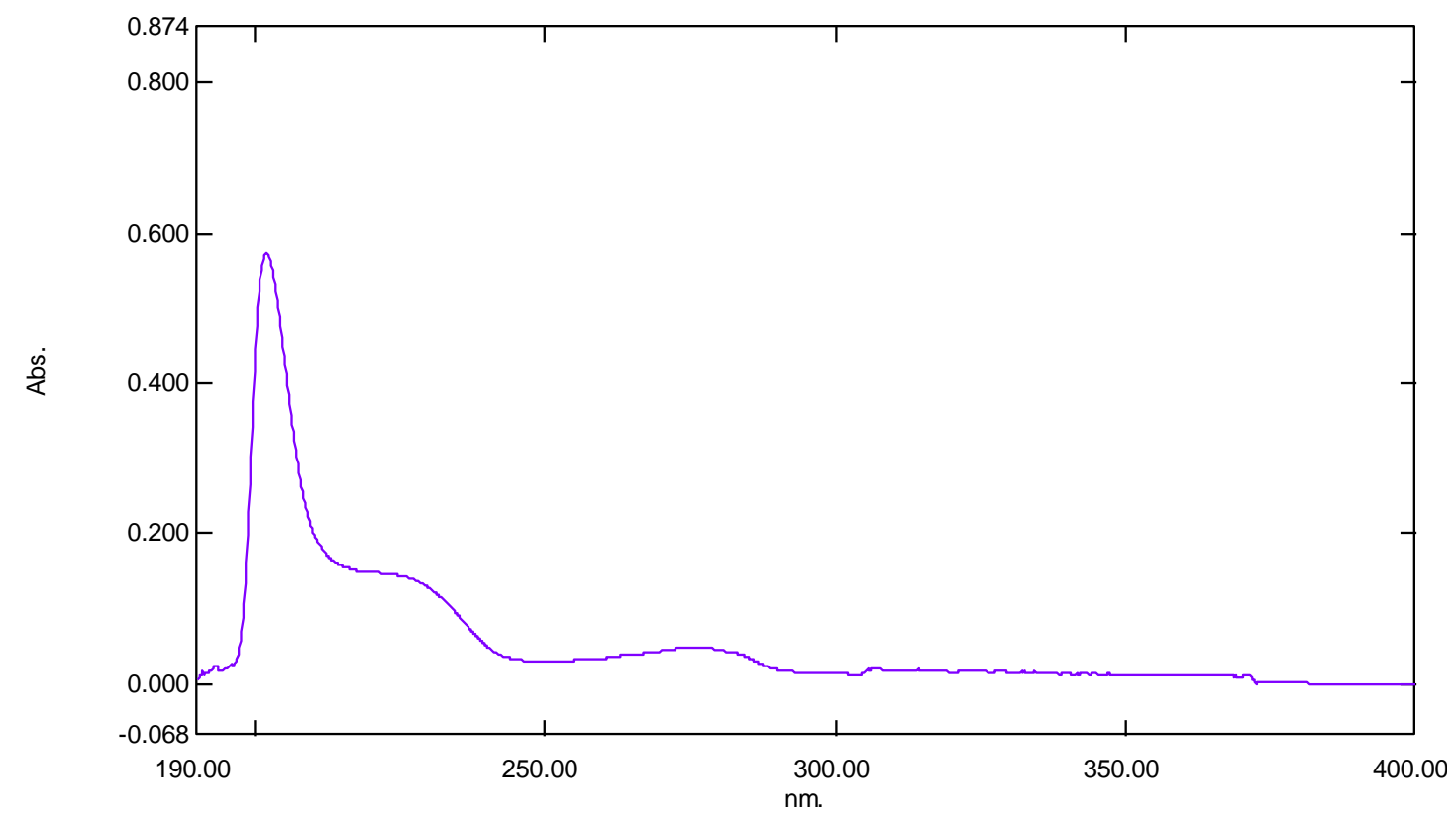

Figure S6. UV Spectrum of Compound 1 


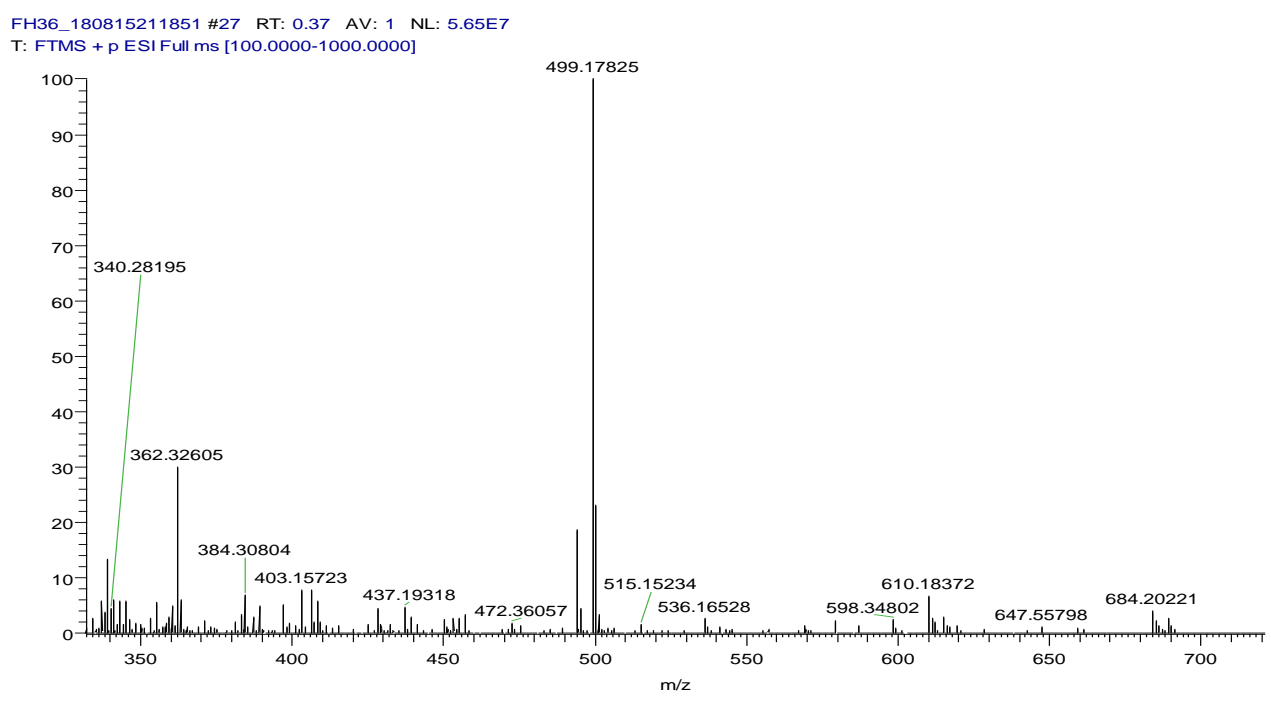

Elemental composition search on mass 499.17913

\begin{tabular}{c|r|r|r|l}
$\mathrm{m} / \mathrm{z}=494.18000-504.17825$ \\
$\mathrm{~m} / \mathrm{z}$ & Theo. Mass & $\begin{array}{c}\text { Delta } \\
(\mathrm{mmu})\end{array}$ & $\begin{array}{r}\text { RDB } \\
\text { equiv. }\end{array}$ & \multicolumn{2}{c}{ Composition } \\
499.17825 & 499.17860 & -0.35 & 5.5 & $\mathrm{C}_{21} \mathrm{H}_{32} \mathrm{O}_{12} \mathrm{Na}$ \\
& 499.18100 & -2.75 & 8.5 & $\mathrm{C}_{23} \mathrm{H}_{31} \mathrm{O}_{12}$ \\
& 499.17513 & 3.12 & 17.5 & $\mathrm{C}_{30} \mathrm{H}_{27} \mathrm{O}_{7}$ \\
& 499.17272 & 5.53 & 14.5 & $\mathrm{C}_{28} \mathrm{H}_{28} \mathrm{O}_{7} \mathrm{Na}$ \\
& 499.16575 & 12.50 & 4.5 & $\mathrm{C}_{19} \mathrm{H}_{31} \mathrm{O}_{15}$ \\
& 499.16334 & 14.91 & 1.5 & $\mathrm{C}_{17} \mathrm{H}_{32} \mathrm{O}_{15} \mathrm{Na}$ \\
& 499.19385 & -15.60 & 9.5 & $\mathrm{C}_{25} \mathrm{H}_{32} \mathrm{O}_{9} \mathrm{Na}$ \\
& 499.19626 & -18.01 & 12.5 & $\mathrm{C}_{27} \mathrm{H}_{31} \mathrm{O}_{9}$ \\
& 499.15987 & 18.38 & 13.5 & $\mathrm{C}_{26} \mathrm{H}_{27} \mathrm{O}_{10}$ \\
& 499.15747 & 20.78 & 10.5 & $\mathrm{C}_{24} \mathrm{H}_{28} \mathrm{O}_{10} \mathrm{Na}$
\end{tabular}

Figure 7. HRESIMS Spectrum of Compound 1

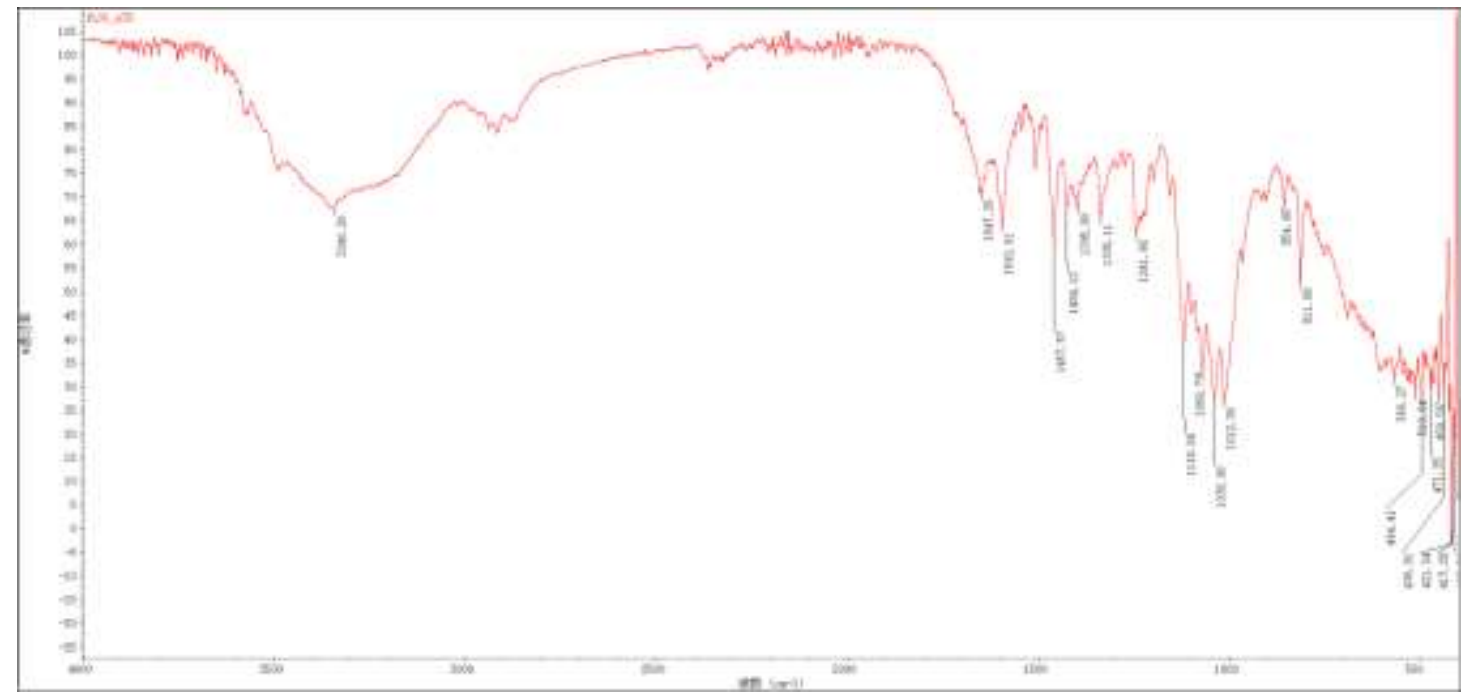

Figure 8. IR Spectrum of Compound 1 


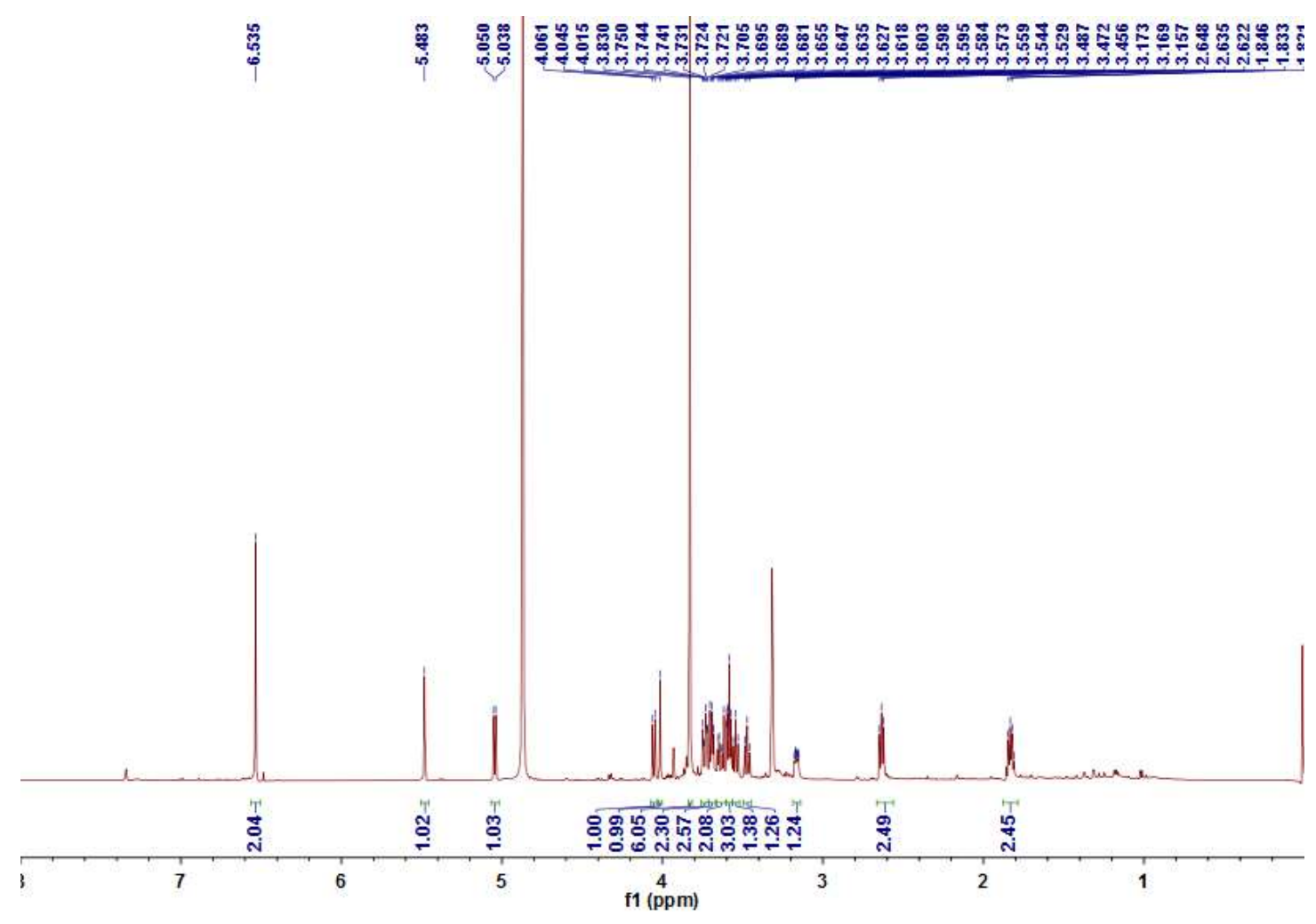

Figure S9. ${ }^{1} \mathrm{H}-\mathrm{NMR}$ Spectrum of Compound 2 in Methanol- $d_{4}$

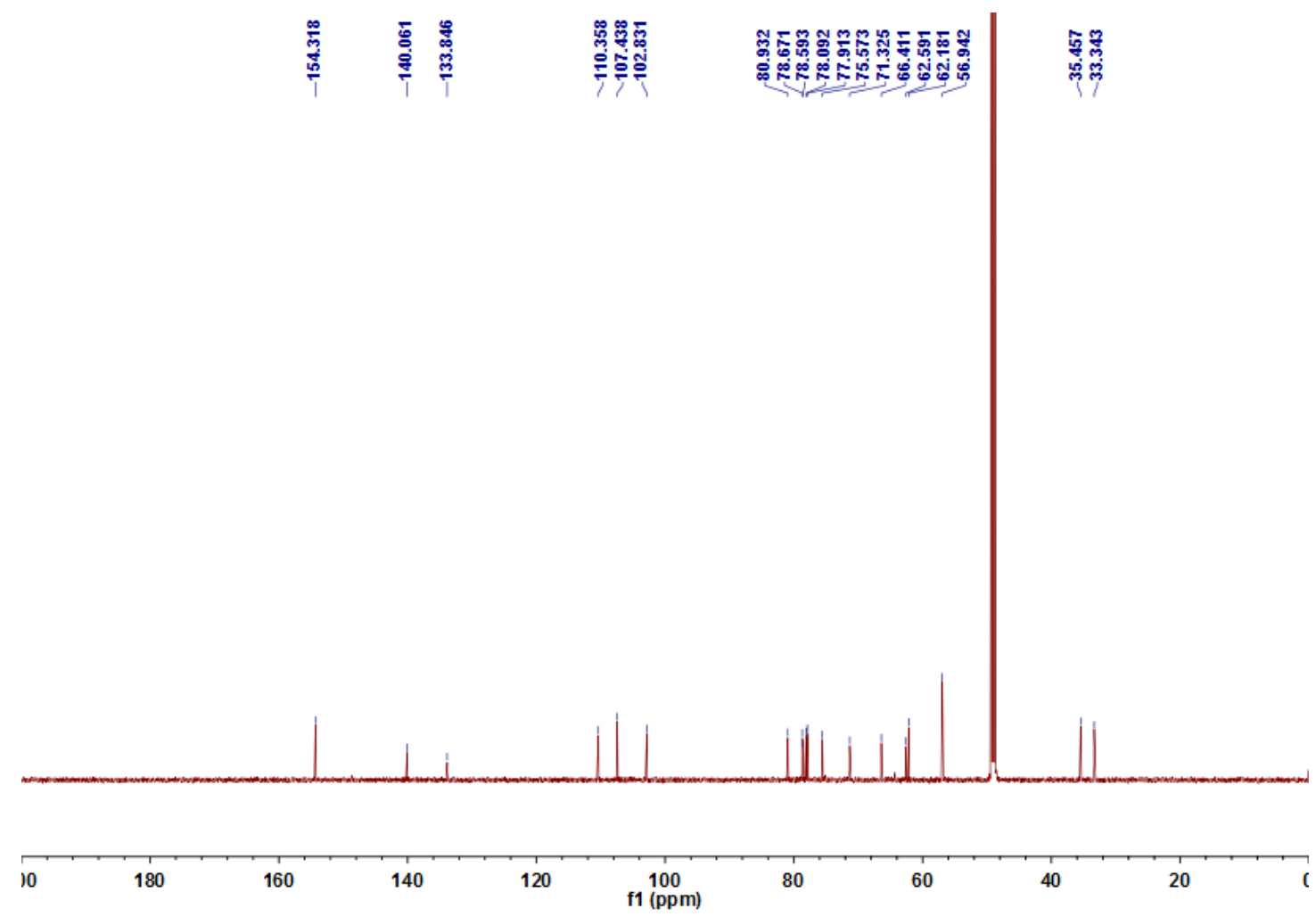

Figure S10. ${ }^{13}$ C-NMR Spectrum of Compound 2 in Methanol- $d_{4}$ 


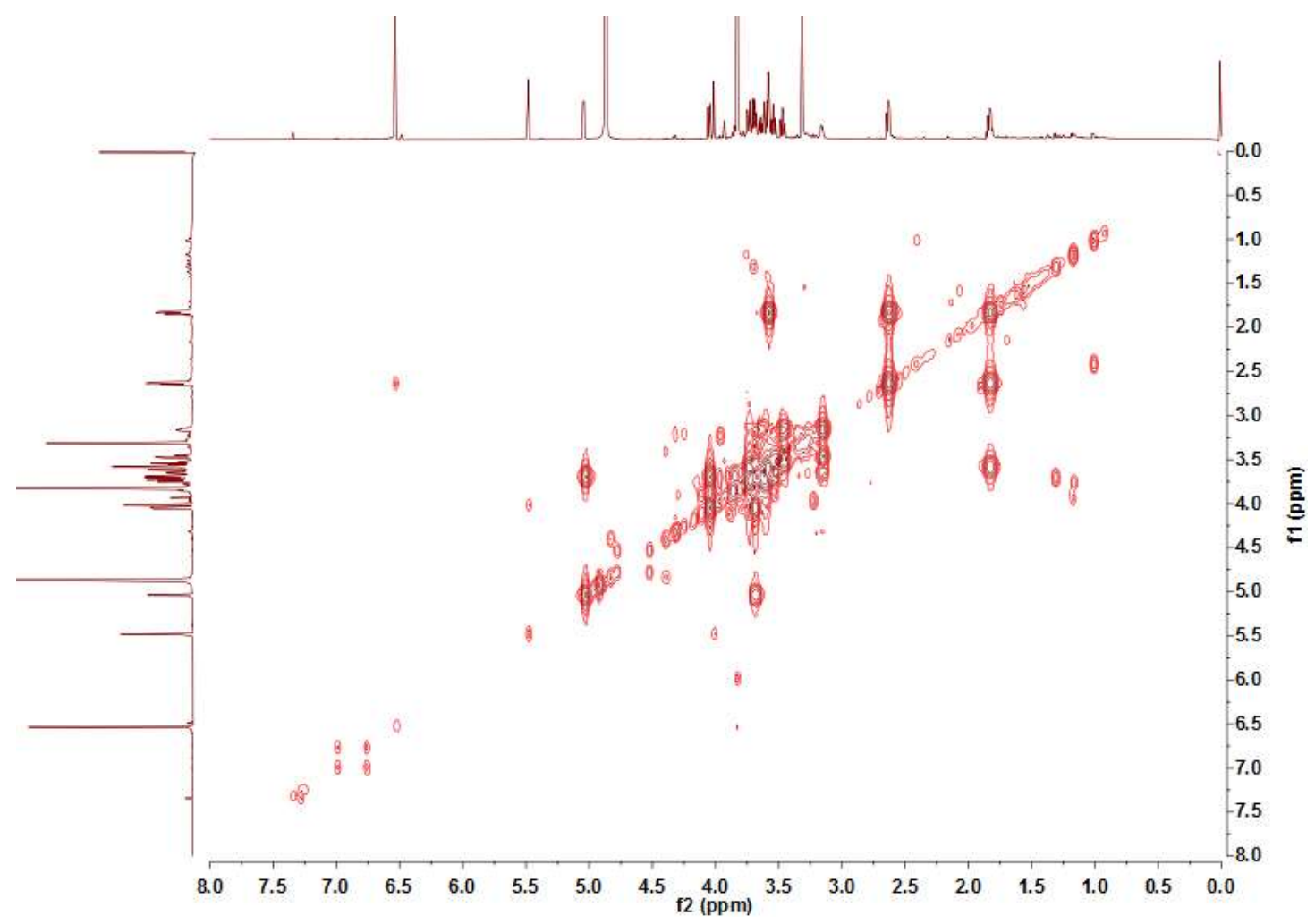

Figure S11. ${ }^{1} \mathrm{H}-{ }^{1} \mathrm{H}$ COSY Spectrum of Compound 2 in Methanol- $d_{4}$

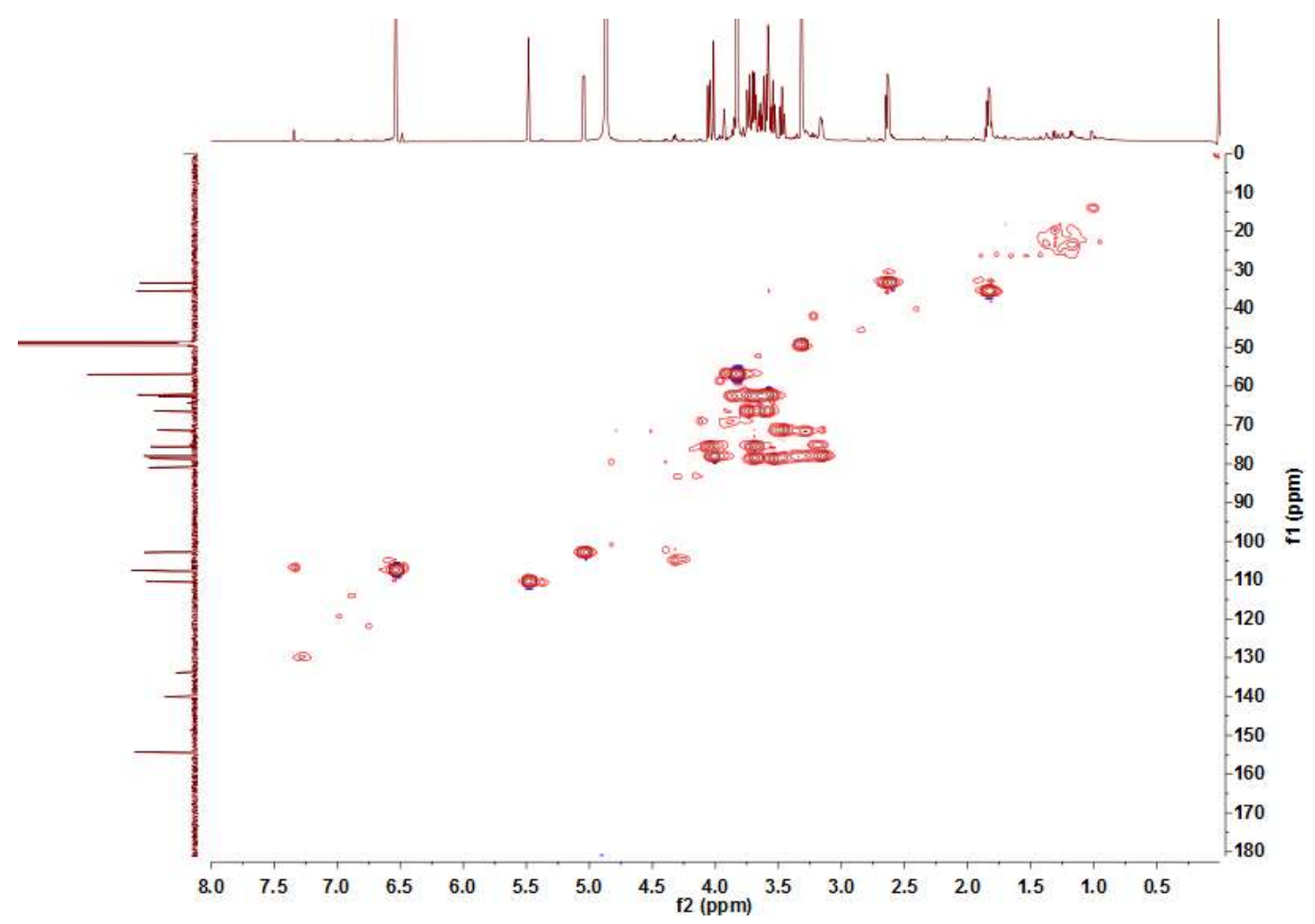

Figure S12. HSQC Spectrum of Compound 2 in Methanol- $d_{4}$ 


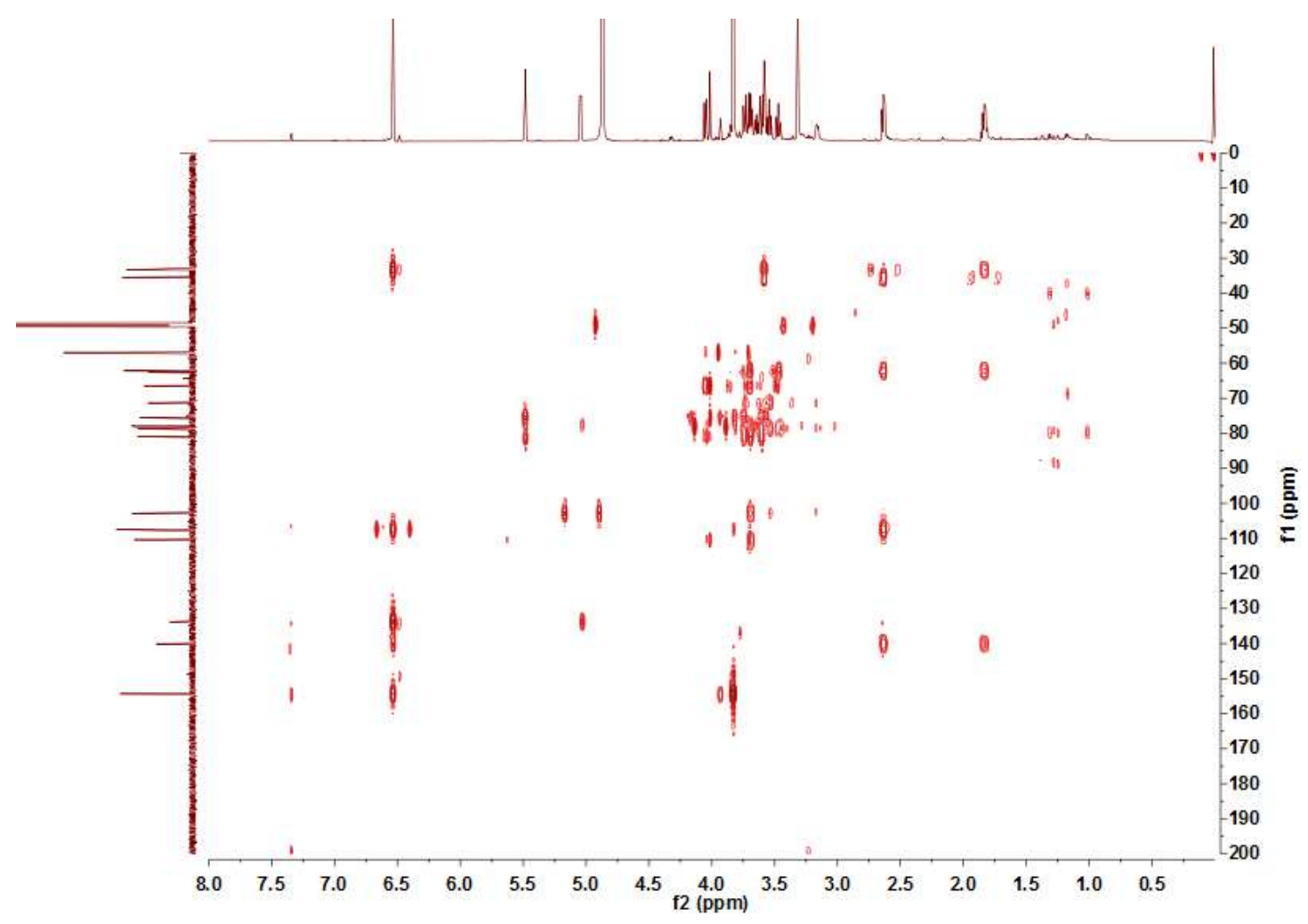

Figure S13. HMBC Spectrum of Compound 2 in Methanol- $d_{4}$

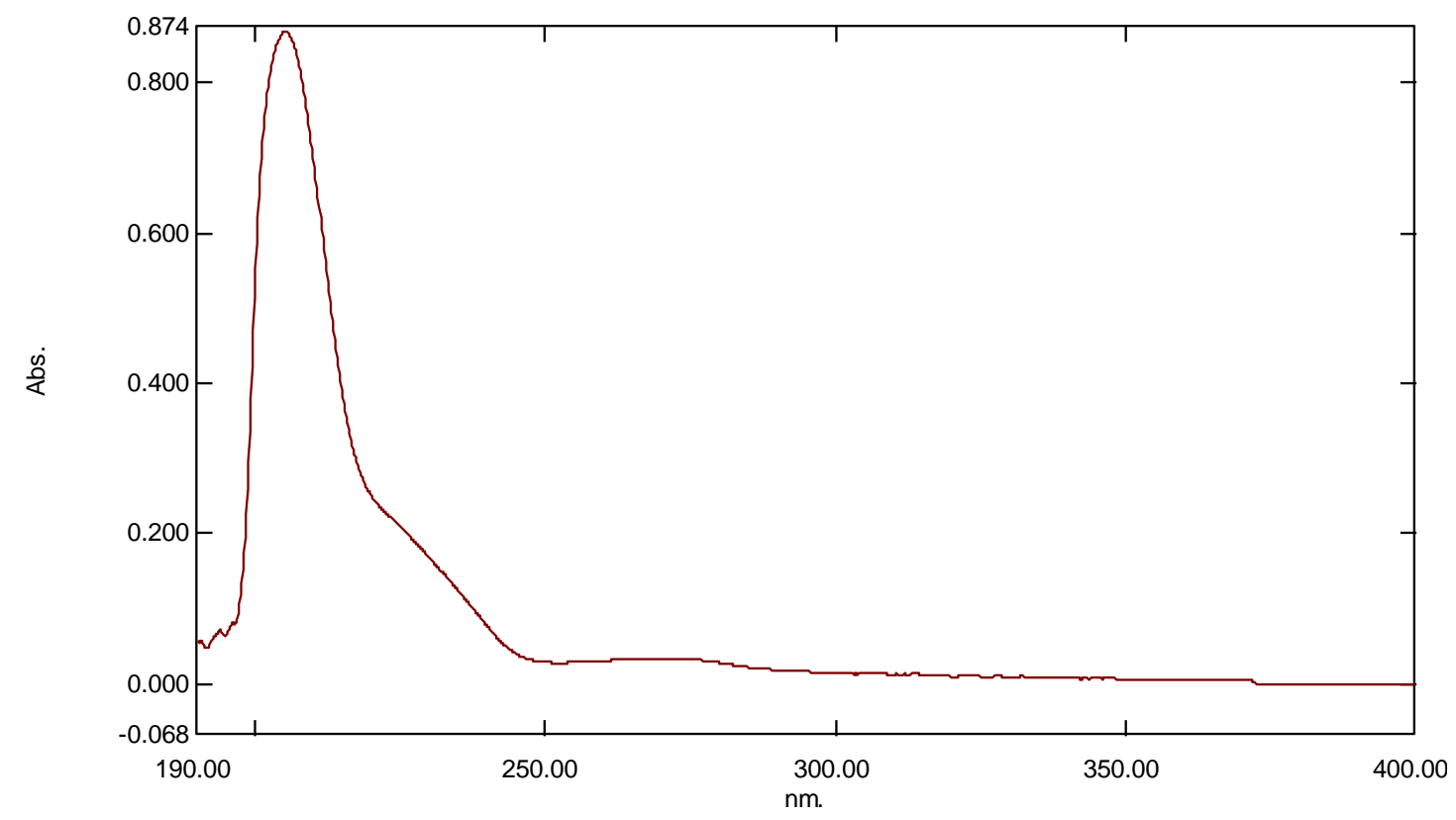

Figure S14. UV Spectrum of Compound 2 


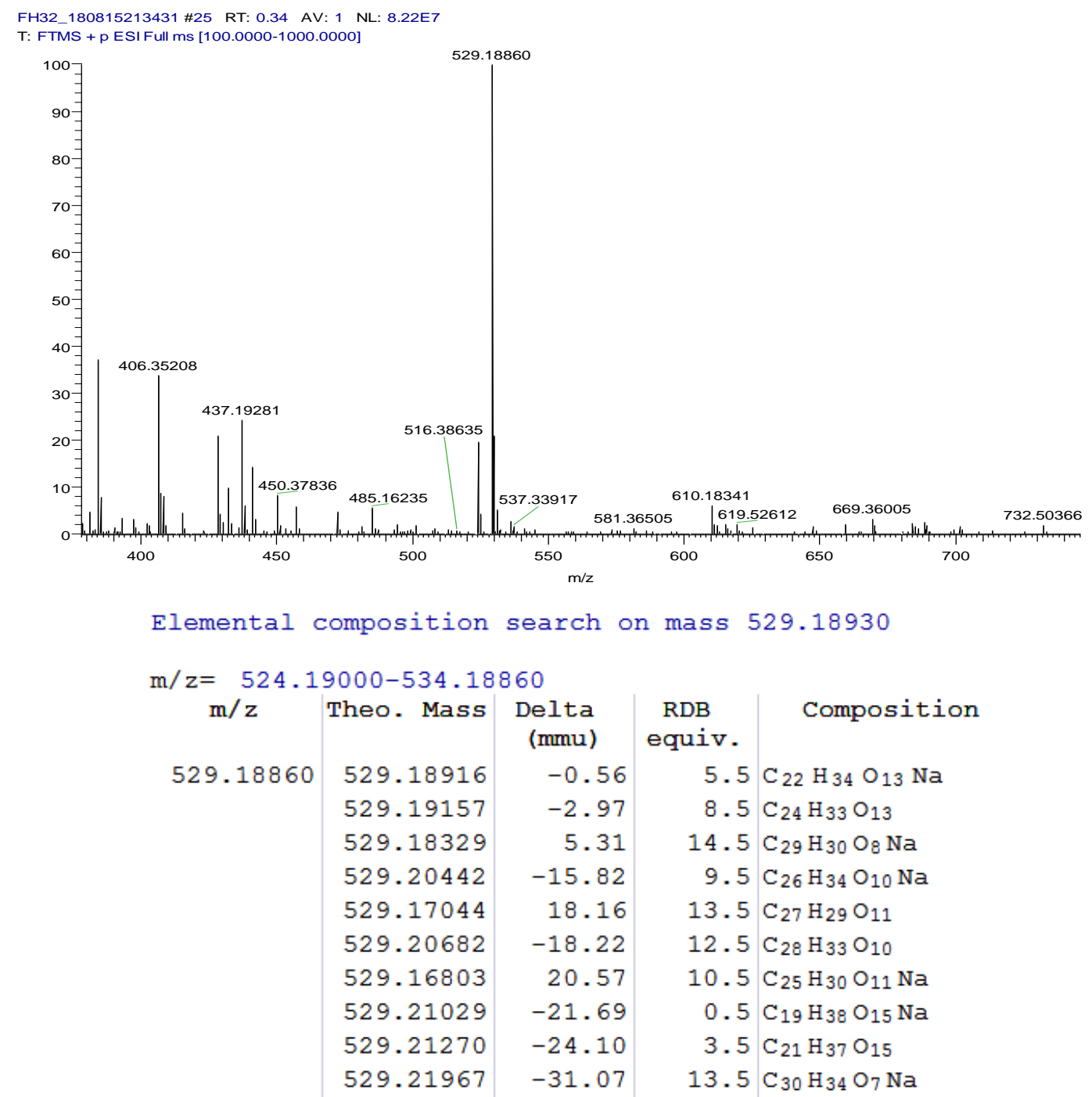

Figure 15. HRESIMS Spectrum of Compound 2

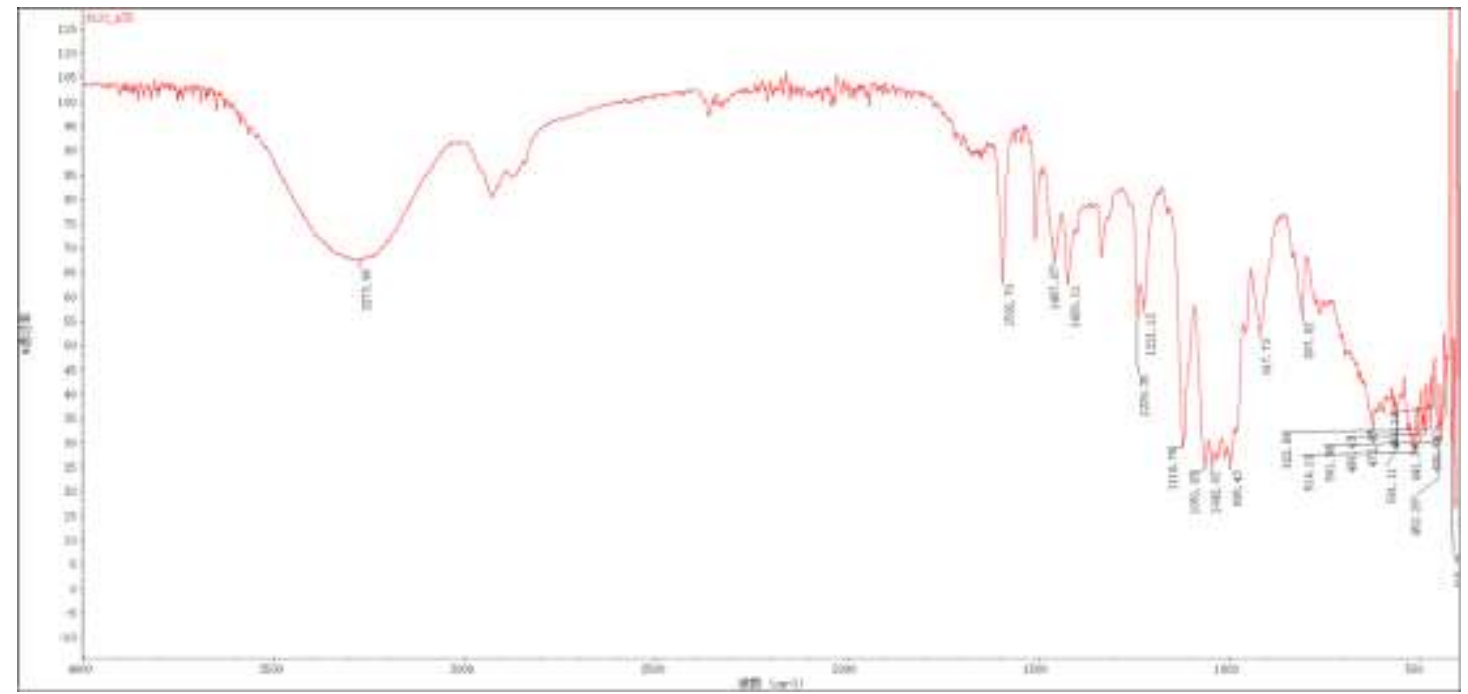

Figure 16. IR Spectrum of Compound 2 


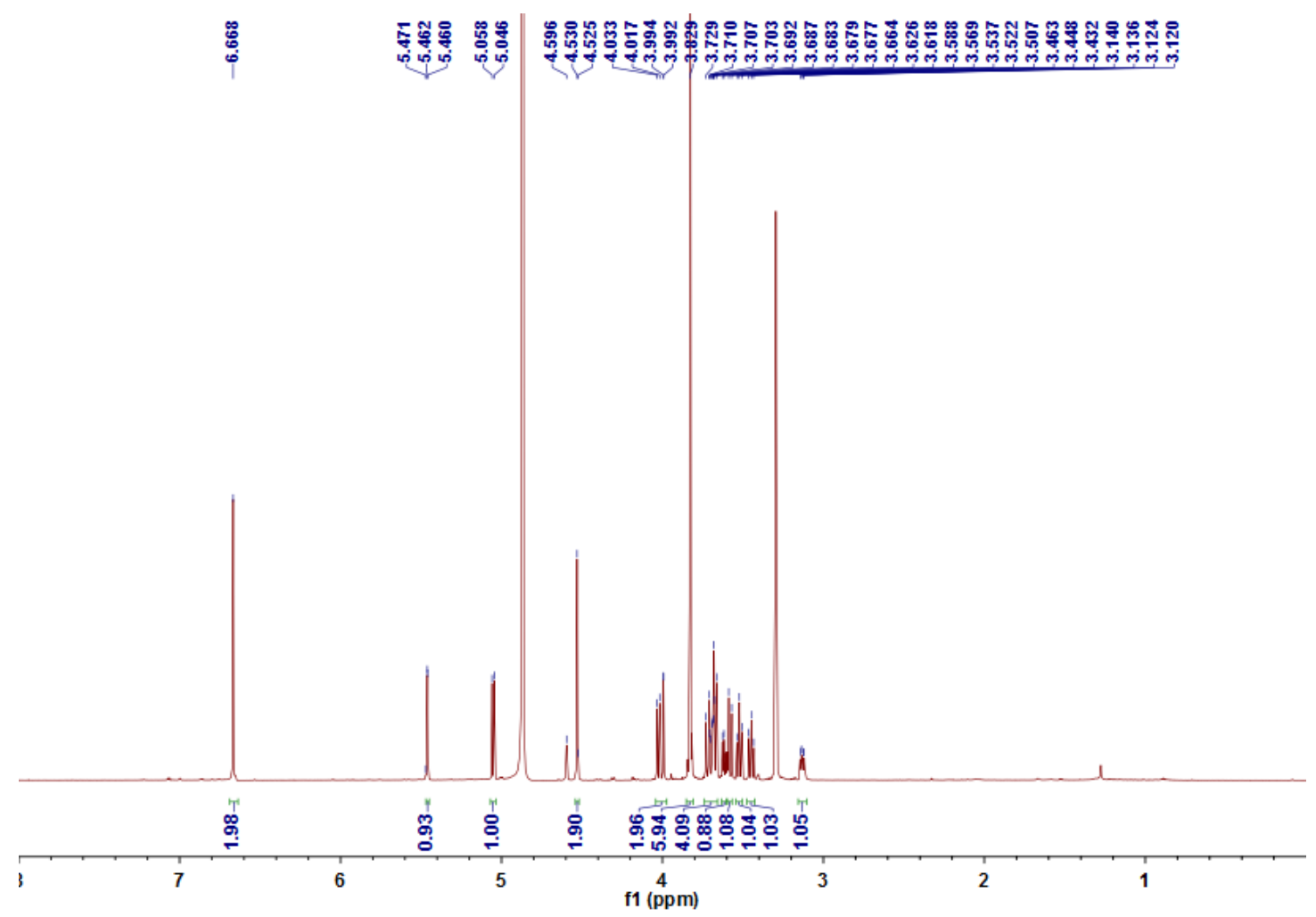

Figure S17. ${ }^{1} \mathrm{H}-\mathrm{NMR}$ Spectrum of Compound 3 in Methanol- $d_{4}$

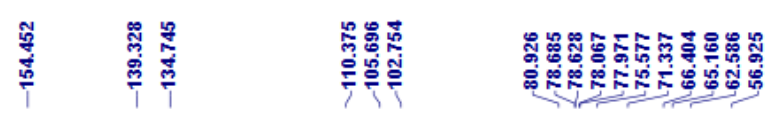

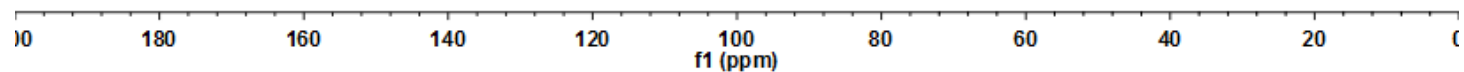

Figure S18. ${ }^{13} \mathrm{C}-\mathrm{NMR}$ Spectrum of Compound 3 in Methanol- $d_{4}$ 


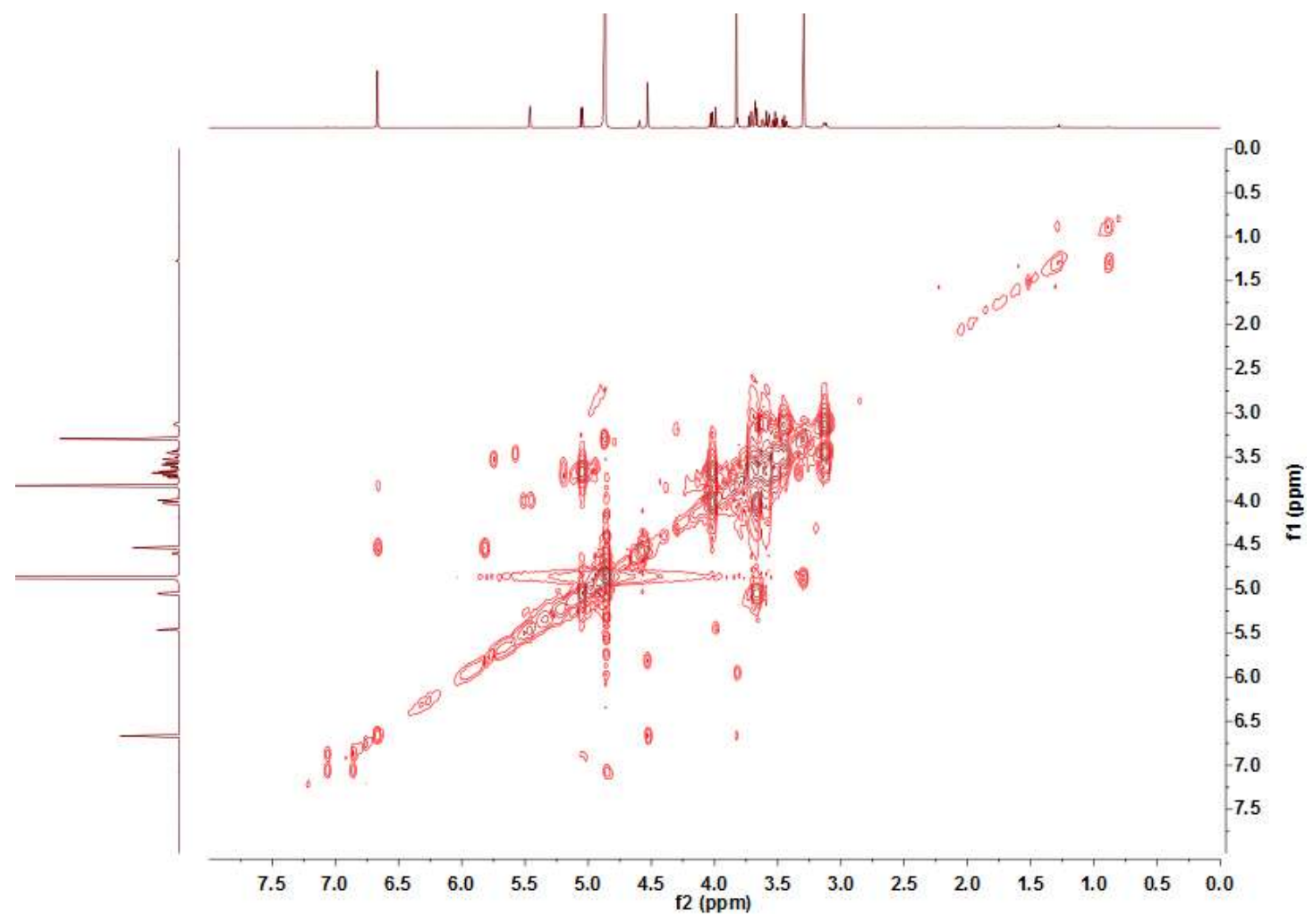

Figure S19. ${ }^{1} \mathrm{H}-{ }^{1} \mathrm{H}$ COSY Spectrum of Compound 3 in Methanol- $d_{4}$

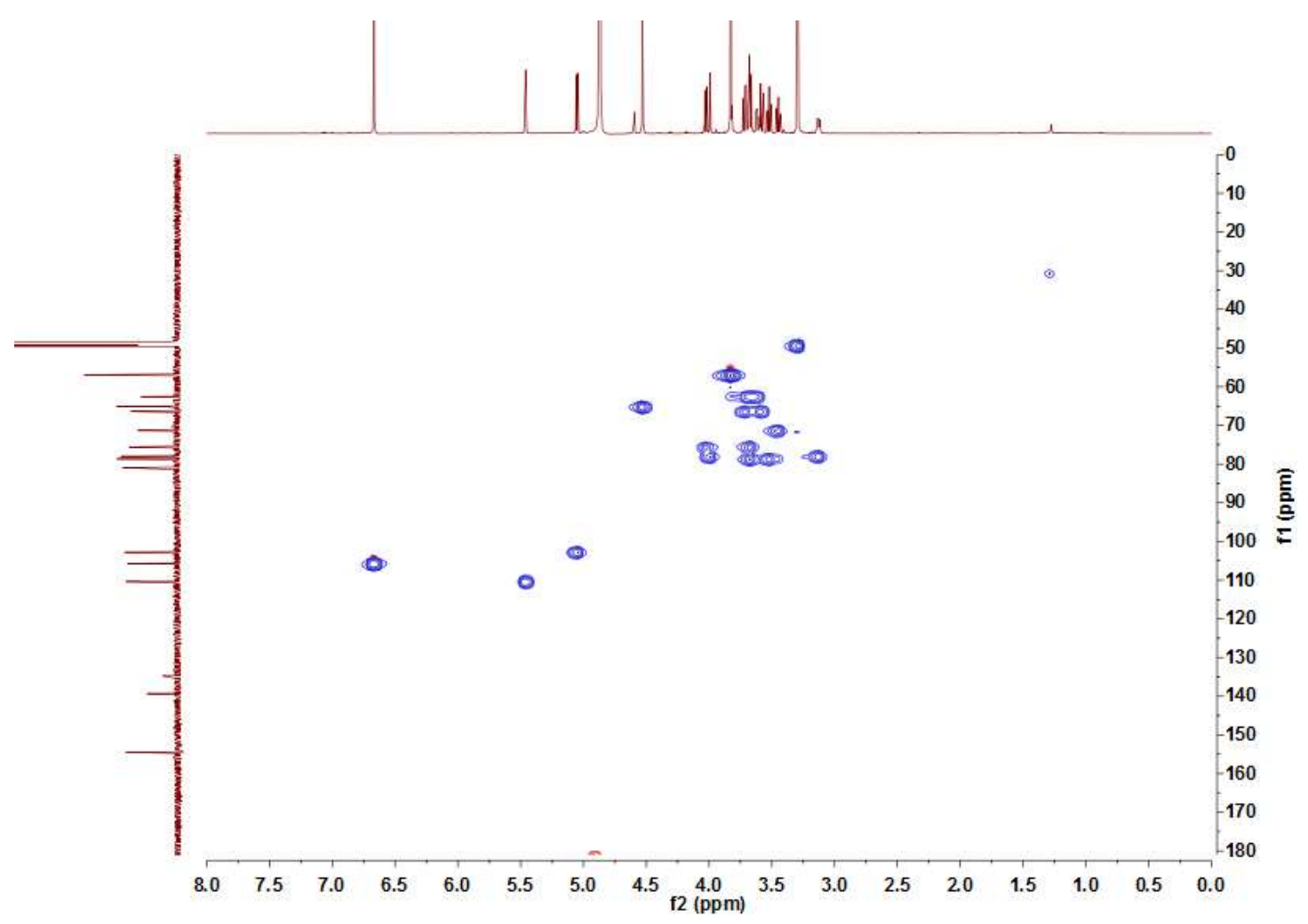

Figure S20. HSQC Spectrum of Compound 3 in Methanol- $d_{4}$ 


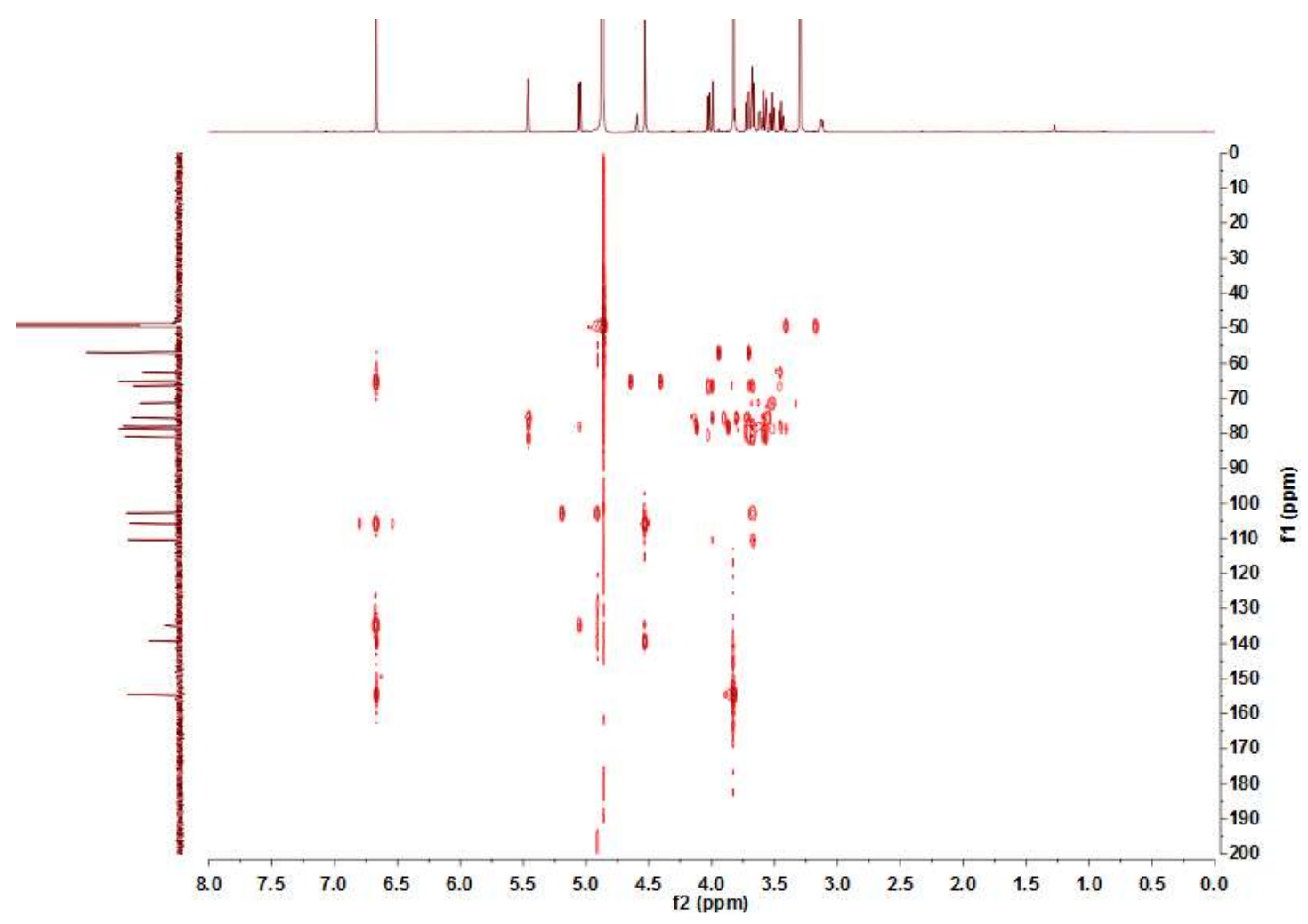

Figure S21. HMBC Spectrum of Compound 3 in Methanol- $d_{4}$

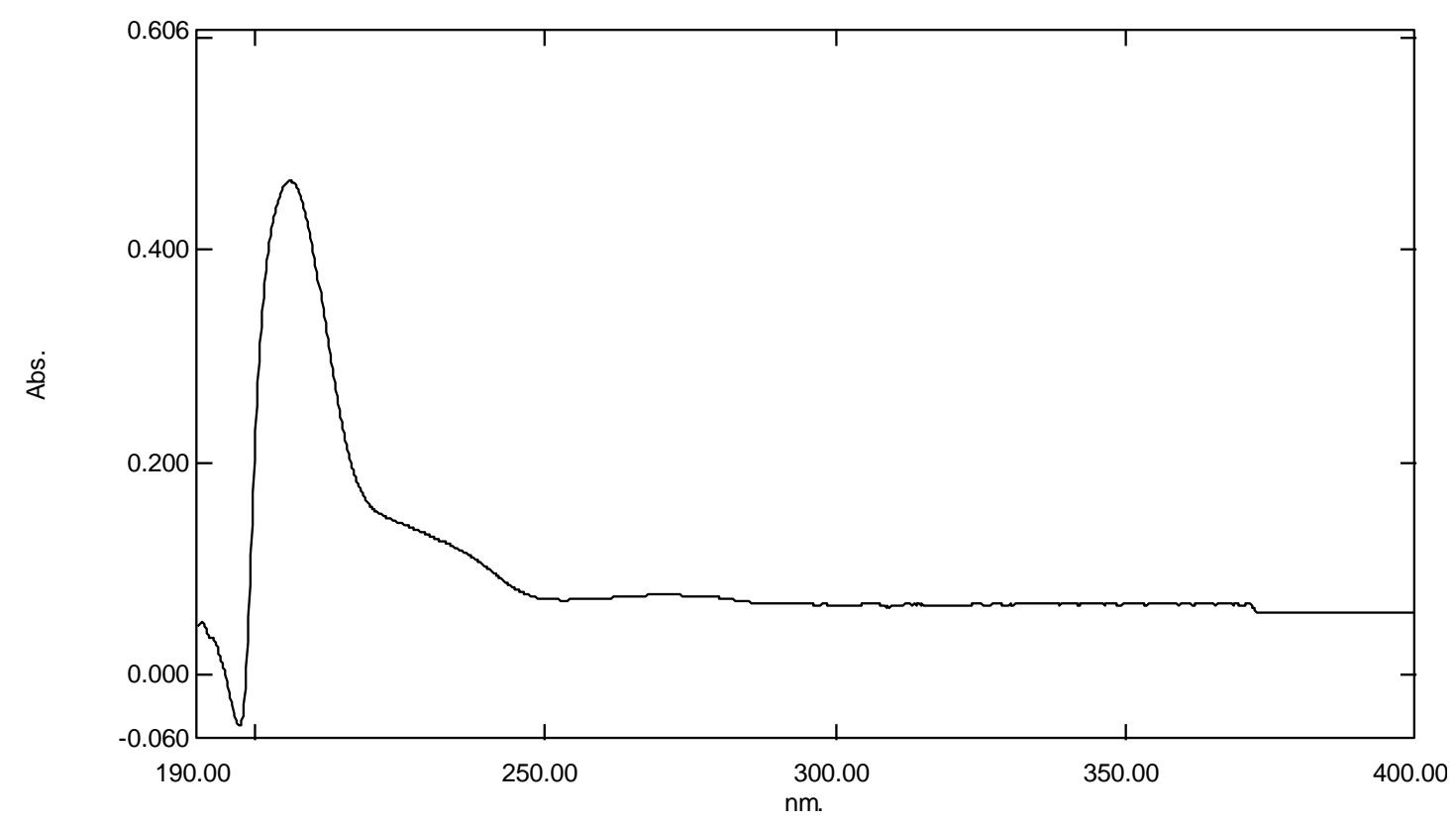

Figure S22. UV Spectrum of Compound 3 


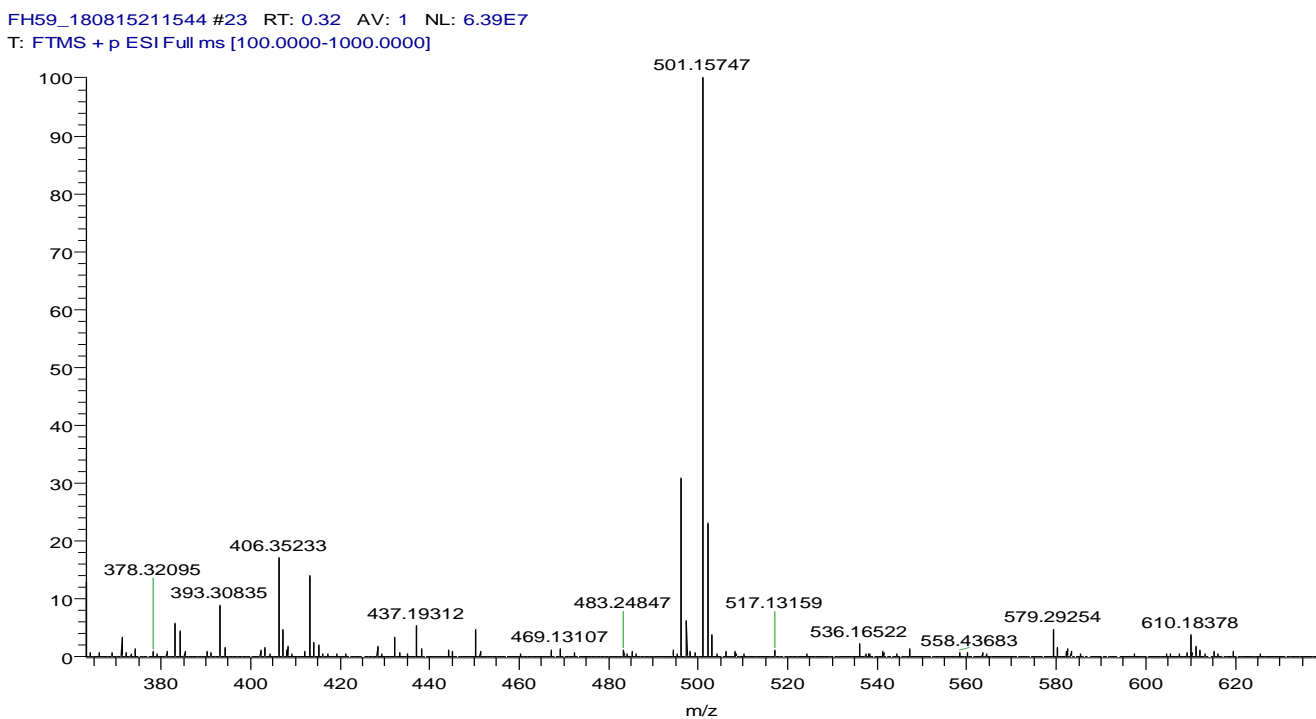

Elemental composition search on mass 501.15873

\begin{tabular}{c|l|r|r|l}
$\mathrm{m} / \mathrm{z}=496.16000-506.15747$ \\
$\mathrm{~m} / \mathrm{z}$ & Theo. Mass & $\begin{array}{c}\text { Delta } \\
\text { (mmu) }\end{array}$ & $\begin{array}{r}\text { RDB } \\
\text { equiv. }\end{array}$ & \multicolumn{1}{c}{ Composition } \\
501.15747 & 501.15786 & -0.39 & 5.5 & $\mathrm{C}_{20} \mathrm{H}_{30} \mathrm{O}_{13} \mathrm{Na}$ \\
& 501.16027 & -2.80 & 8.5 & $\mathrm{C}_{22} \mathrm{H}_{29} \mathrm{O}_{13}$ \\
& 501.15439 & 3.08 & 17.5 & $\mathrm{C}_{29} \mathrm{H}_{25} \mathrm{O}_{8}$ \\
& 501.15199 & 5.48 & 14.5 & $\mathrm{C}_{27} \mathrm{H}_{26} \mathrm{O}_{8} \mathrm{Na}$ \\
& 501.17312 & -15.65 & 9.5 & $\mathrm{C}_{24} \mathrm{H}_{30} \mathrm{O}_{10} \mathrm{Na}$ \\
& 501.17552 & -18.05 & 12.5 & $\mathrm{C}_{26} \mathrm{H}_{29} \mathrm{O}_{10}$ \\
& 501.13914 & 18.33 & 13.5 & $\mathrm{C}_{25} \mathrm{H}_{25} \mathrm{O}_{11}$ \\
& 501.13673 & 20.74 & 10.5 & $\mathrm{C}_{23} \mathrm{H}_{26} \mathrm{O}_{11} \mathrm{Na}$ \\
& 501.17899 & -21.52 & 0.5 & $\mathrm{C}_{17} \mathrm{H}_{34} \mathrm{O}_{15} \mathrm{Na}$ \\
& 501.18140 & -23.93 & 3.5 & $\mathrm{C}_{19} \mathrm{H}_{33} \mathrm{O}_{15}$
\end{tabular}

Figure 23. HRESIMS Spectrum of Compound 3

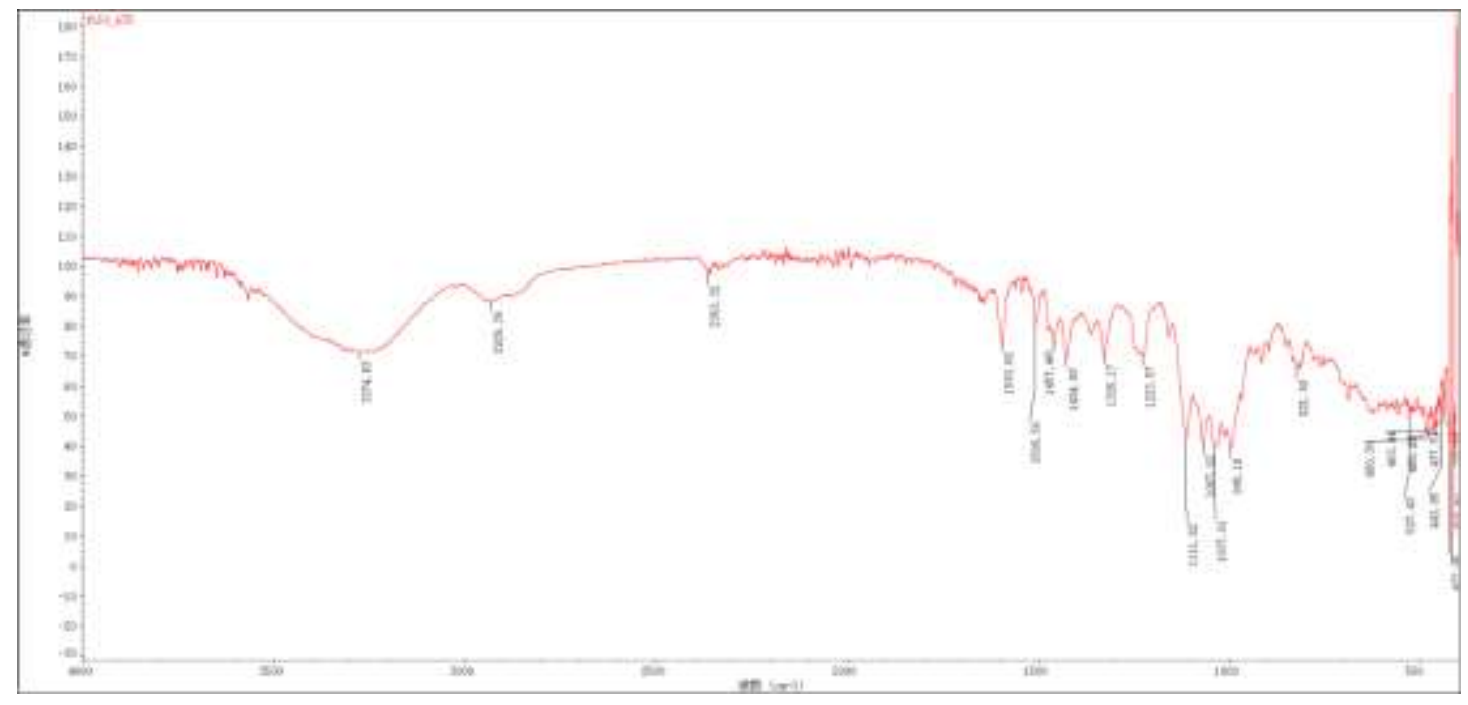

Figure 24. IR Spectrum of Compound 3 


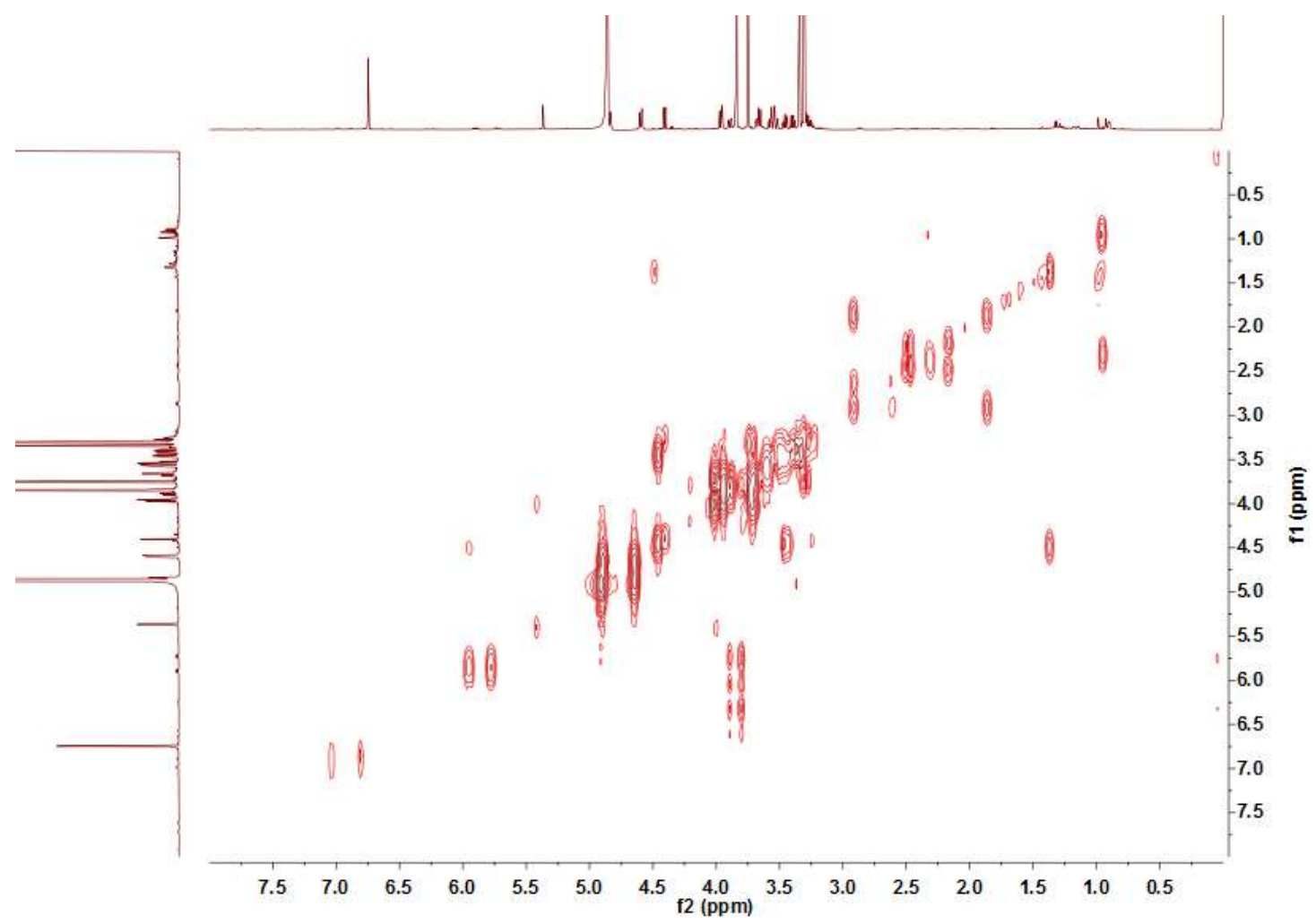

Figure S27. ${ }^{1} \mathrm{H}-{ }^{1} \mathrm{H}$ COSY Spectrum of Compound 4 in Methanol- $d_{4}$

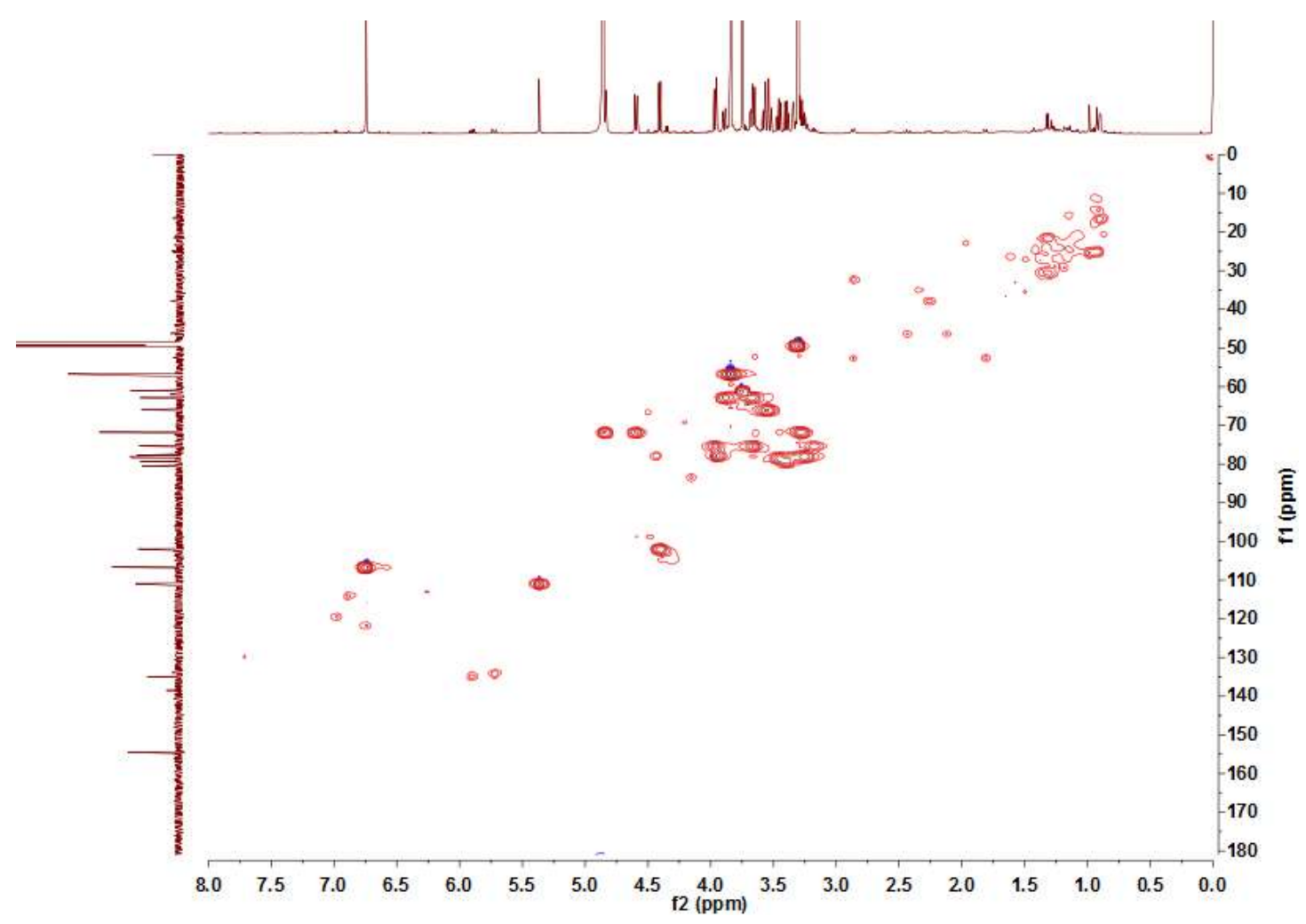

Figure S28. HSQC Spectrum of Compound 4 in Methanol- $d_{4}$ 


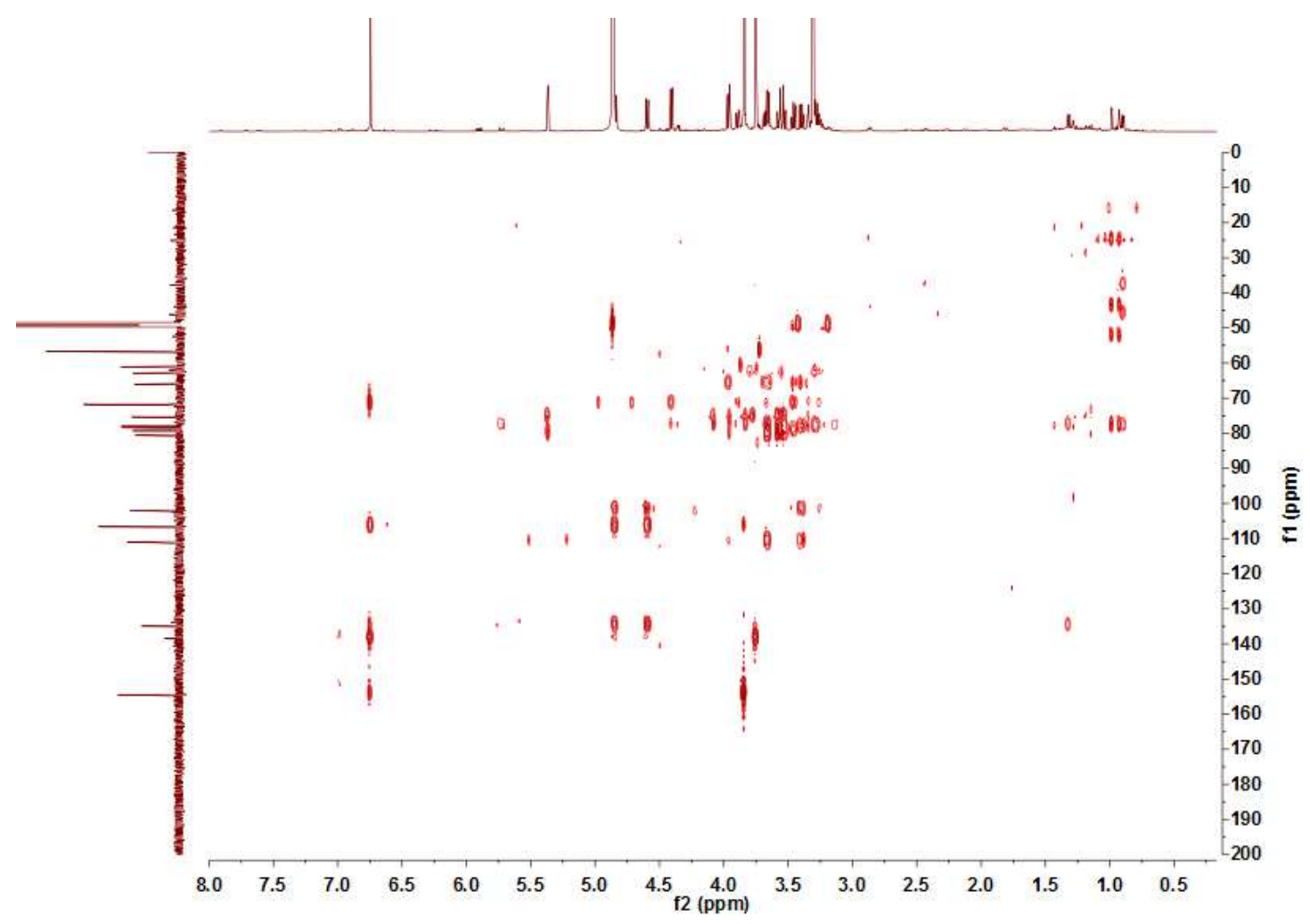

Figure S29. HMBC Spectrum of Compound 4 in Methanol- $d_{4}$

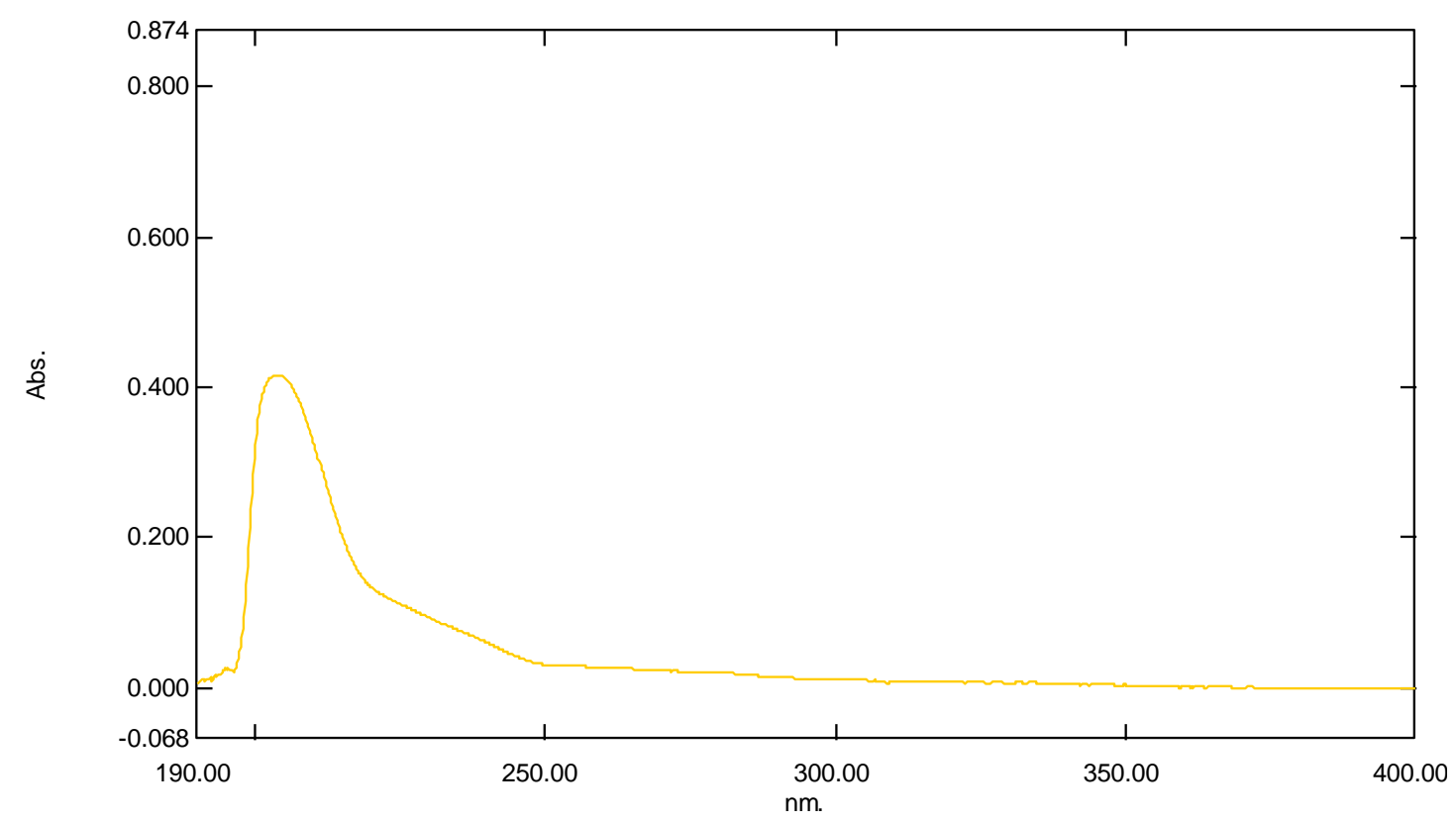

Figure S30. UV Spectrum of Compound 4 
FH10_180815210315 \#33 RT: 0.46 AV: 1 NL: 1.41E7

T: FTMS + p ESI Full ms [100.0000-1000.0000]

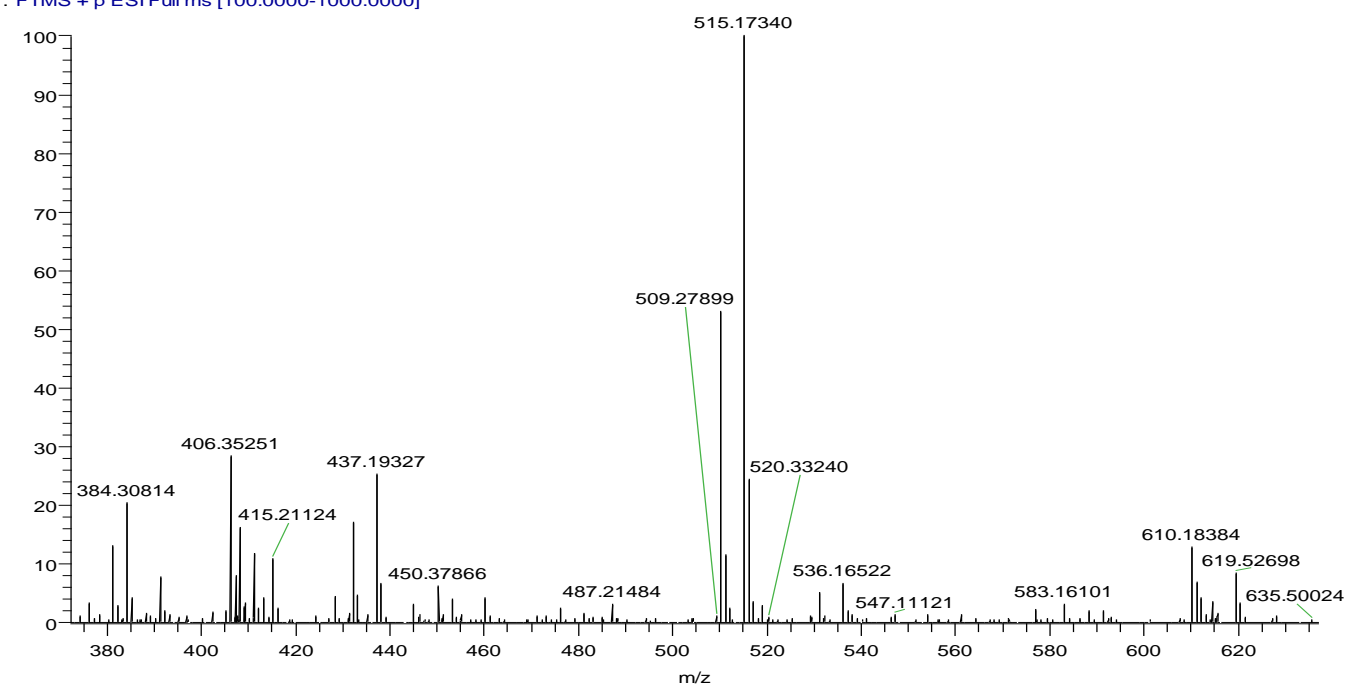

Elemental composition search on mass 515.17170

\begin{tabular}{c|r|r|r|l}
$\mathrm{m} / \mathrm{z}=\begin{array}{l}510.17340-520.17000 \\
\mathrm{~m} / \mathrm{z}\end{array}$ & Theo. Mass & $\begin{array}{c}\text { Delta } \\
(\mathrm{mmu})\end{array}$ & $\begin{array}{r}\text { RDB } \\
\text { equiv. }\end{array}$ & \multicolumn{1}{|l}{ Composition } \\
515.17340 & 515.17351 & -0.11 & 5.5 & $\mathrm{C}_{21} \mathrm{H}_{32} \mathrm{O}_{13} \mathrm{Na}$ \\
& 515.17327 & 0.13 & 6.5 & $\mathrm{C}_{24} \mathrm{H}_{32} \mathrm{O}_{5} \mathrm{Na}_{5}$ \\
& 515.17568 & -2.28 & 9.5 & $\mathrm{C}_{26} \mathrm{H}_{31} \mathrm{O}_{5} \mathrm{Na}_{4}$ \\
& 515.17111 & 2.29 & 2.5 & $\mathrm{C}_{19} \mathrm{H}_{33} \mathrm{O}_{13} \mathrm{Na}_{2}$ \\
& 515.17592 & -2.52 & 8.5 & $\mathrm{C}_{23} \mathrm{H}_{31} \mathrm{O}_{13}$ \\
& 515.17087 & 2.53 & 3.5 & $\mathrm{C}_{22} \mathrm{H}_{33} \mathrm{O}_{5} \mathrm{Na}_{6}$ \\
515.17004 & 3.36 & 17.5 & $\mathrm{C}_{30} \mathrm{H}_{27} \mathrm{O}_{8}$ \\
& 515.17808 & -4.68 & 12.5 & $\mathrm{C}_{28} \mathrm{H}_{30} \mathrm{O}_{5} \mathrm{Na}_{3}$ \\
& 515.16870 & 4.70 & -0.5 & $\mathrm{C}_{17} \mathrm{H}_{34} \mathrm{O}_{13} \mathrm{Na}_{3}$ \\
515.16846 & 4.94 & 0.5 & $\mathrm{C}_{20} \mathrm{H}_{34} \mathrm{O}_{5} \mathrm{Na}_{7}$
\end{tabular}

Figure 31. HRESIMS Spectrum of Compound 4

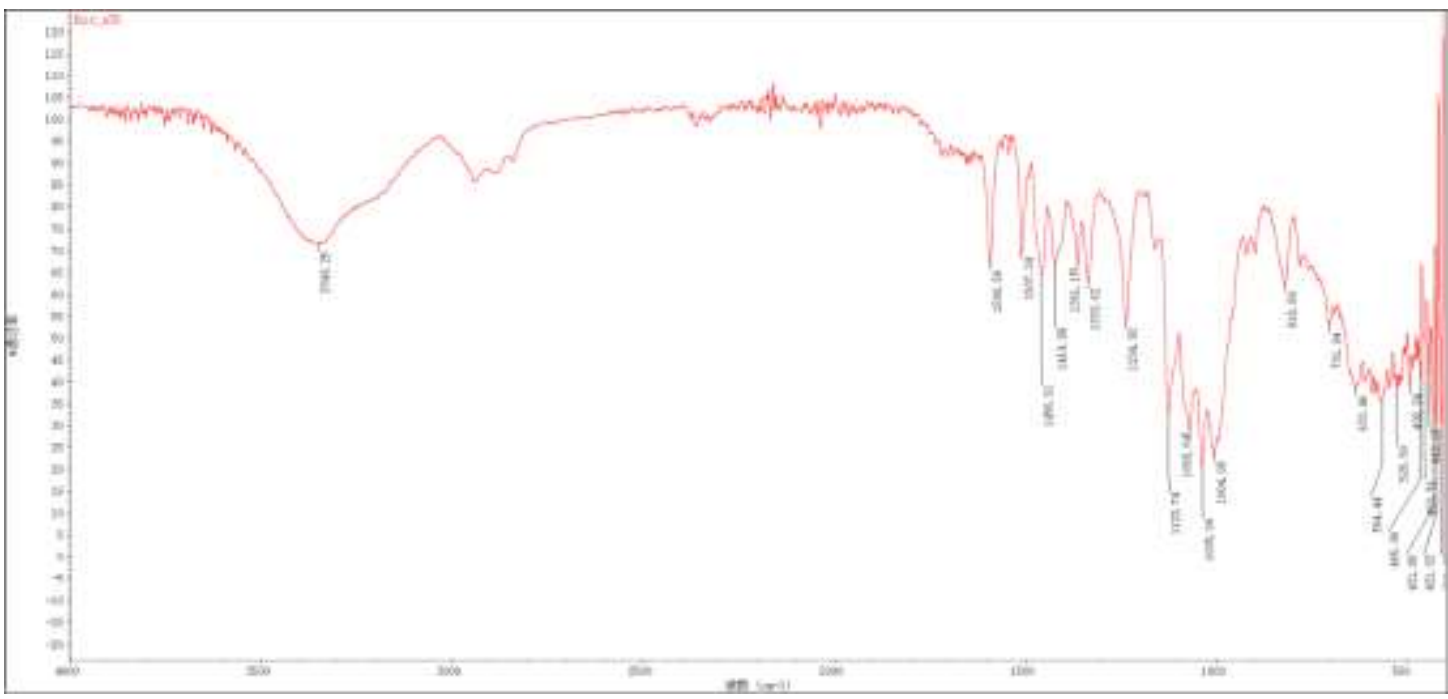

Figure 32. IR Spectrum of Compound 4 


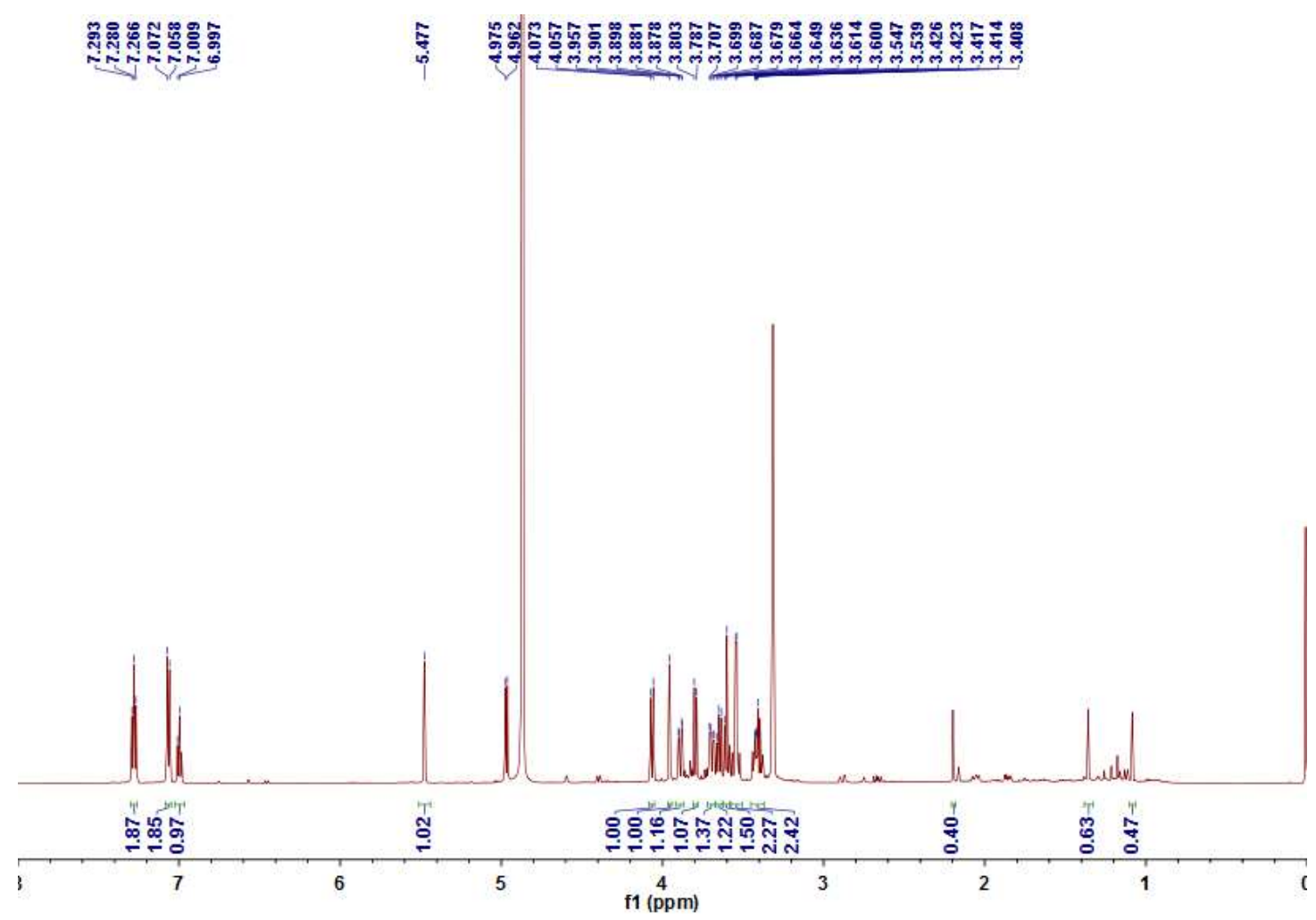

Figure S33. ${ }^{1} \mathrm{H}-\mathrm{NMR}$ Spectrum of Compound 5 in Methanol- $d_{4}$

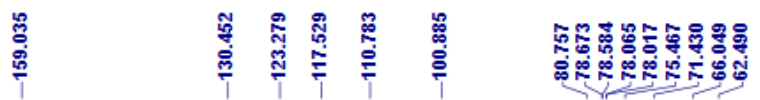

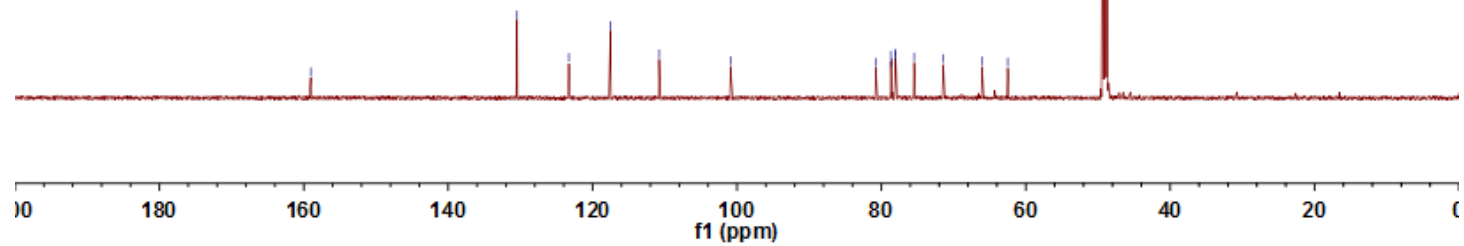

Figure S34. ${ }^{13} \mathrm{C}-\mathrm{NMR}$ Spectrum of Compound 5 in Methanol- $d_{4}$ 


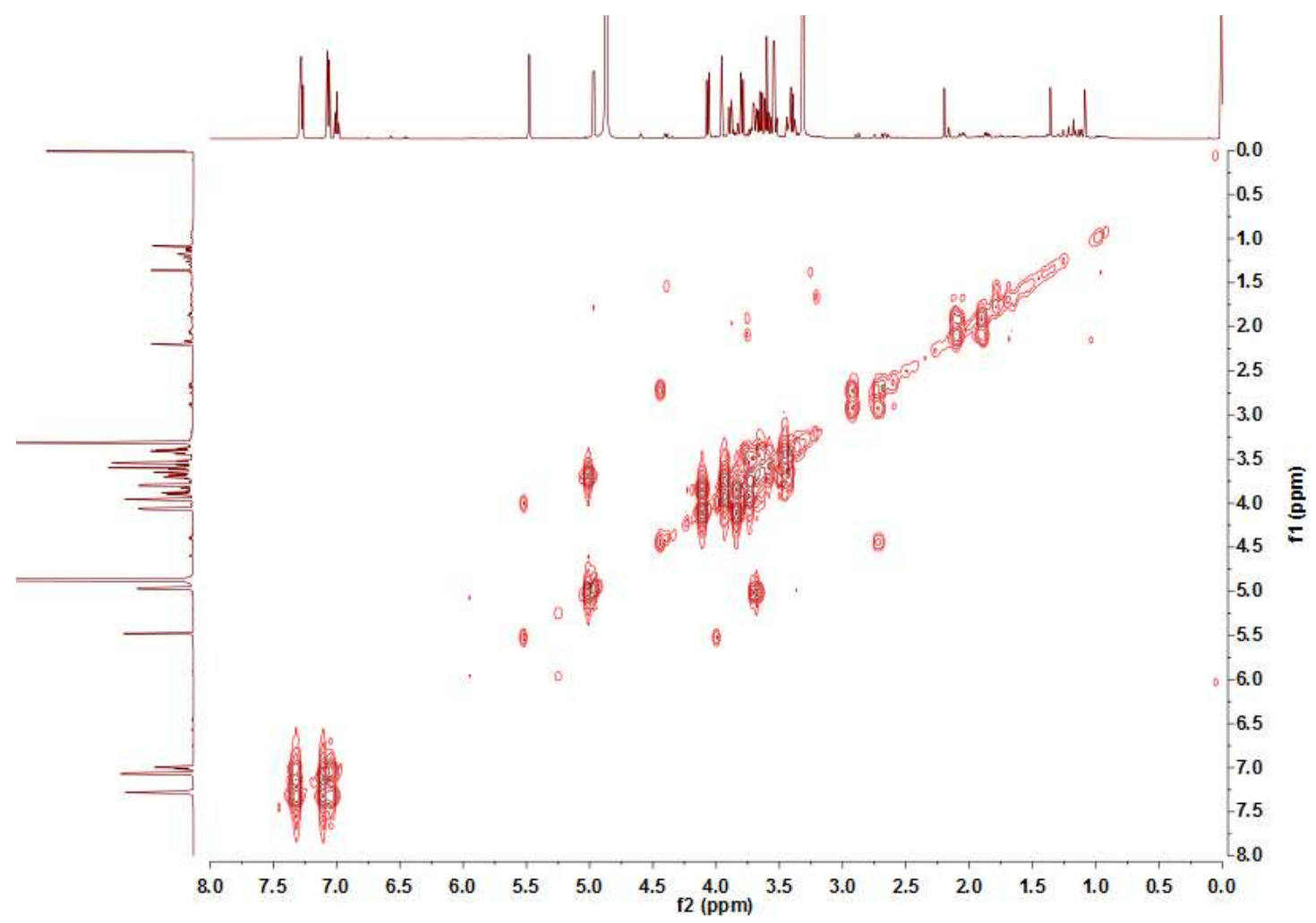

Figure S35. ${ }^{1} \mathrm{H}-{ }^{1} \mathrm{H}$ COSY Spectrum of Compound 5 in Methanol- $d_{4}$

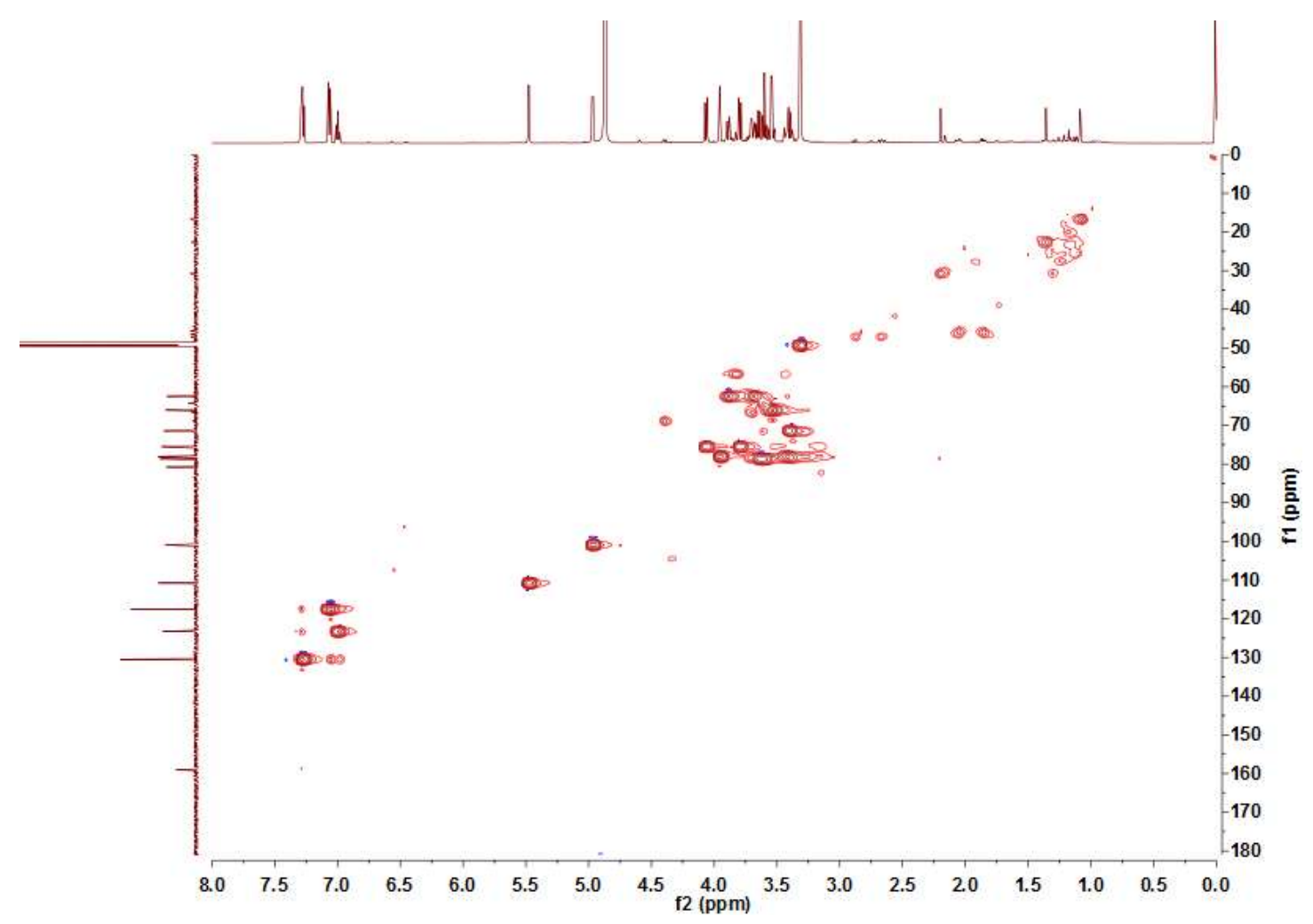

Figure S36. HSQC Spectrum of Compound 5 in Methanol- $d_{4}$ 


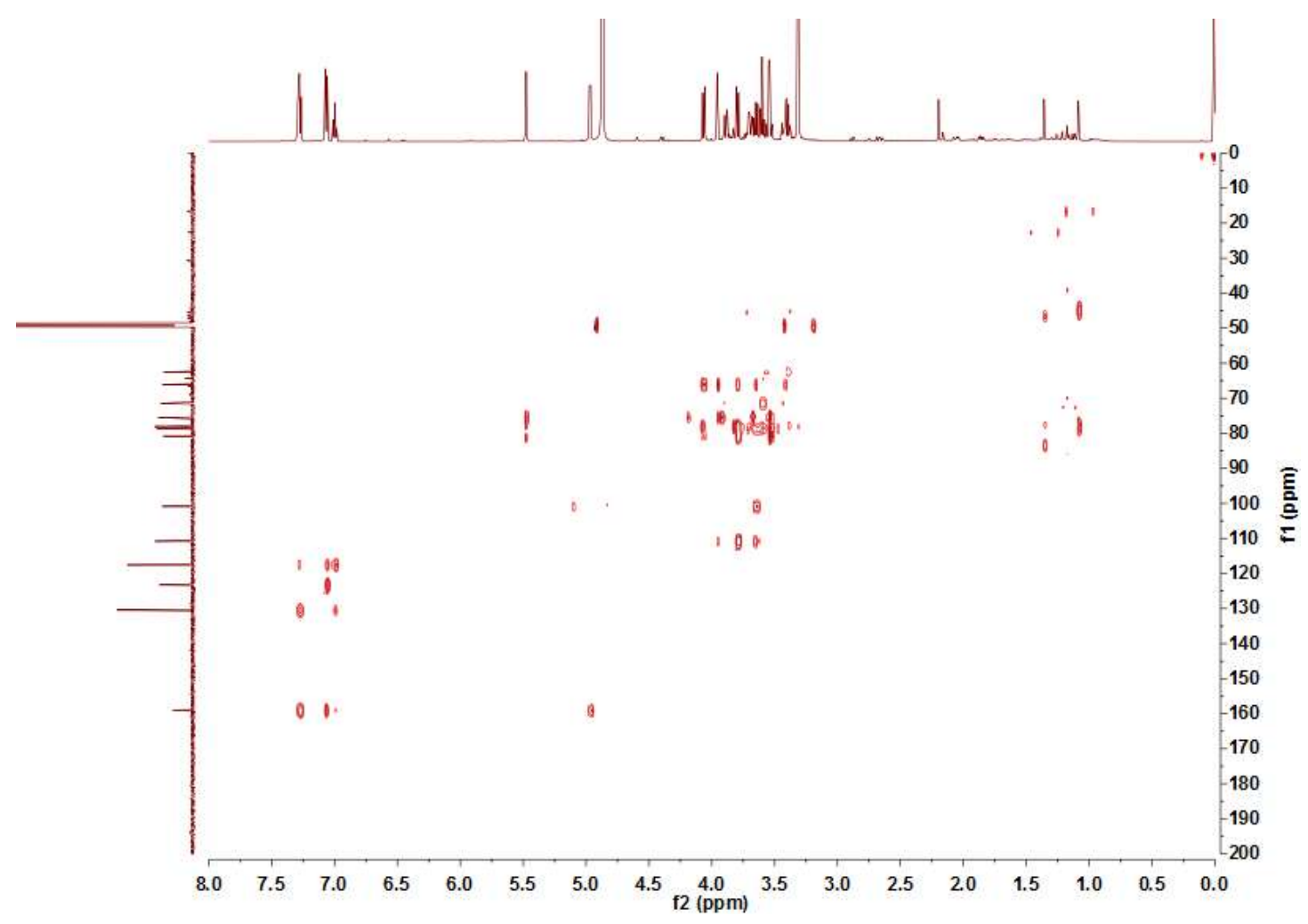

Figure S37. HMBC Spectrum of Compound 5 in Methanol- $d_{4}$

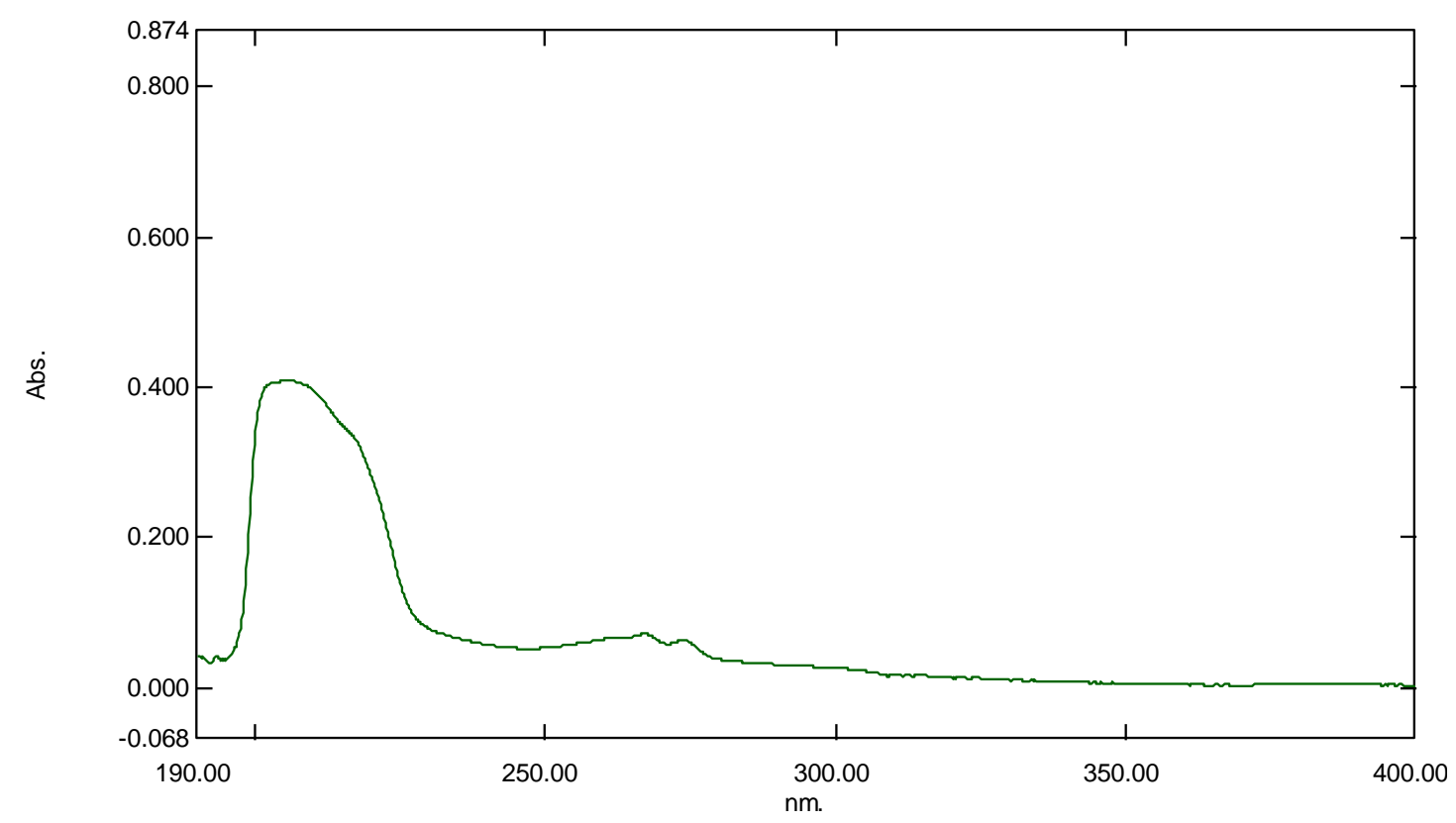

Figure S38. UV Spectrum of Compound 5 


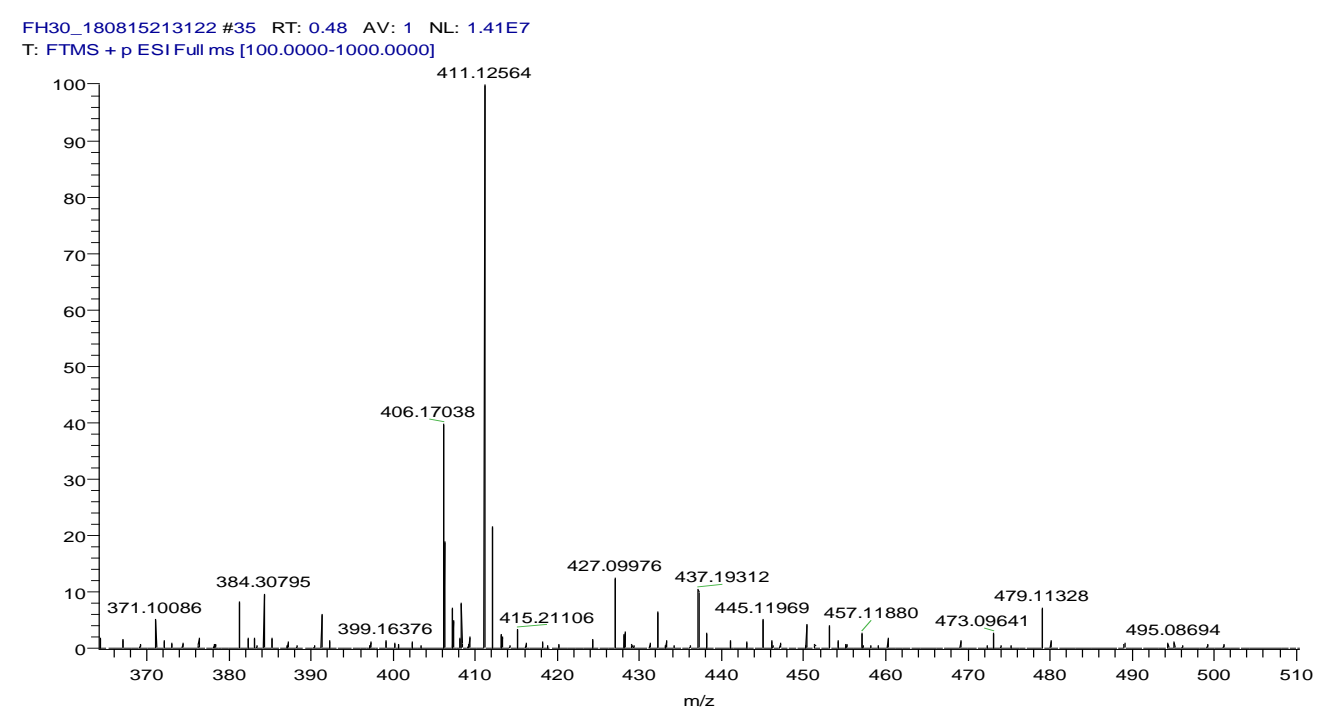

Elemental composition search on mass 411.12782

\begin{tabular}{|c|c|c|c|c|}
\hline $\mathrm{m} / \mathrm{z}$ & Theo. Mass & $\begin{array}{r}\text { Delta } \\
\text { (mmu) }\end{array}$ & $\begin{array}{c}\text { RDB } \\
\text { equiv. }\end{array}$ & Composition \\
\hline \multirow[t]{10}{*}{411.12564} & 411.12617 & -0.53 & 5.5 & $\mathrm{C}_{17} \mathrm{H}_{24} \mathrm{O}_{10} \mathrm{Na}$ \\
\hline & 411.12857 & -2.93 & 8.5 & $\mathrm{C}_{19} \mathrm{H}_{23} \mathrm{O}_{10}$ \\
\hline & 411.12270 & 2.94 & 17.5 & $\mathrm{C}_{26} \mathrm{H}_{19} \mathrm{O}_{5}$ \\
\hline & 411.12029 & 5.35 & 14.5 & $\mathrm{C}_{24} \mathrm{H}_{20} \mathrm{O}_{5} \mathrm{Na}$ \\
\hline & 411.13445 & -8.81 & -0.5 & $\mathrm{C}_{12} \mathrm{H}_{27} \mathrm{O}_{15}$ \\
\hline & 411.13555 & -9.91 & 18.5 & $\mathrm{C}_{28} \mathrm{H}_{20} \mathrm{O}_{2} \mathrm{Na}$ \\
\hline & 411.13796 & $-12 \cdot 32$ & 21.5 & $\mathrm{C}_{30} \mathrm{H}_{19} \mathrm{O}_{2}$ \\
\hline & 411.11332 & 12.32 & 4.5 & $\mathrm{C}_{15} \mathrm{H}_{23} \mathrm{O}_{13}$ \\
\hline & 411.11091 & 14.73 & 1.5 & $\mathrm{C}_{13} \mathrm{H}_{24} \mathrm{O}_{13} \mathrm{Na}$ \\
\hline & 411.14142 & -15.78 & 9.5 & $\mathrm{C}_{21} \mathrm{H}_{24} \mathrm{O}_{7} \mathrm{Na}$ \\
\hline
\end{tabular}

Figure39. HRESIMS Spectrum of Compound 5

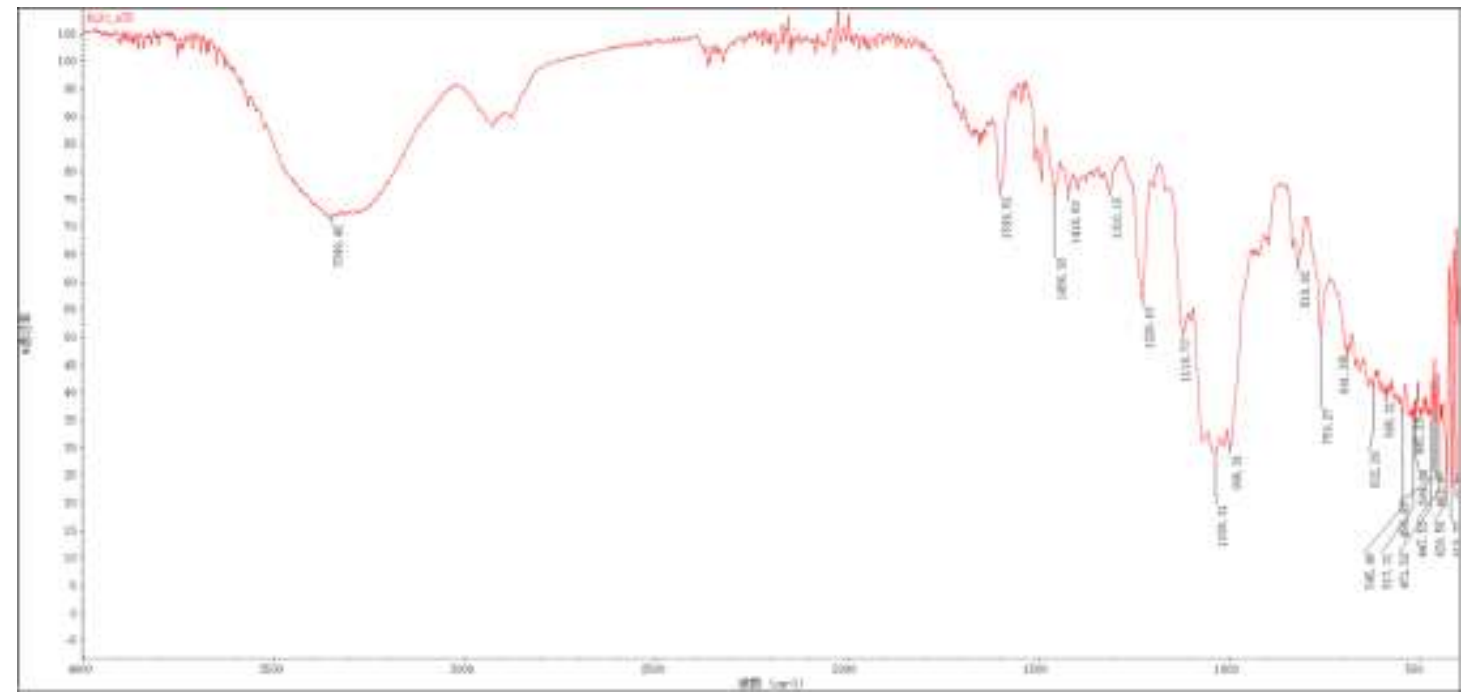

Figure 40. IR Spectrum of Compound 5 


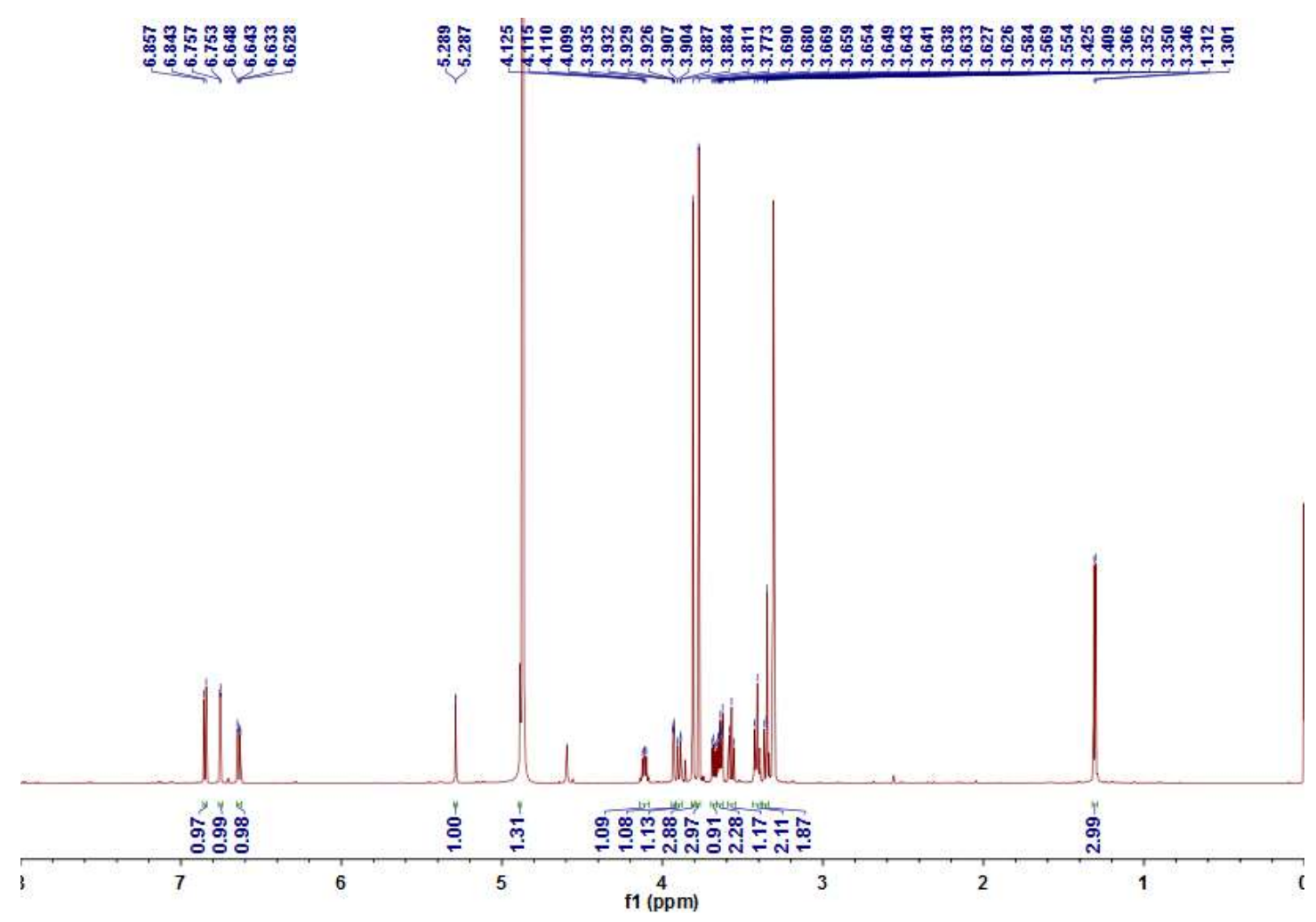

Figure S41. ${ }^{1} \mathrm{H}-\mathrm{NMR}$ Spectrum of Compound 6 in Methanol- $d_{4}$

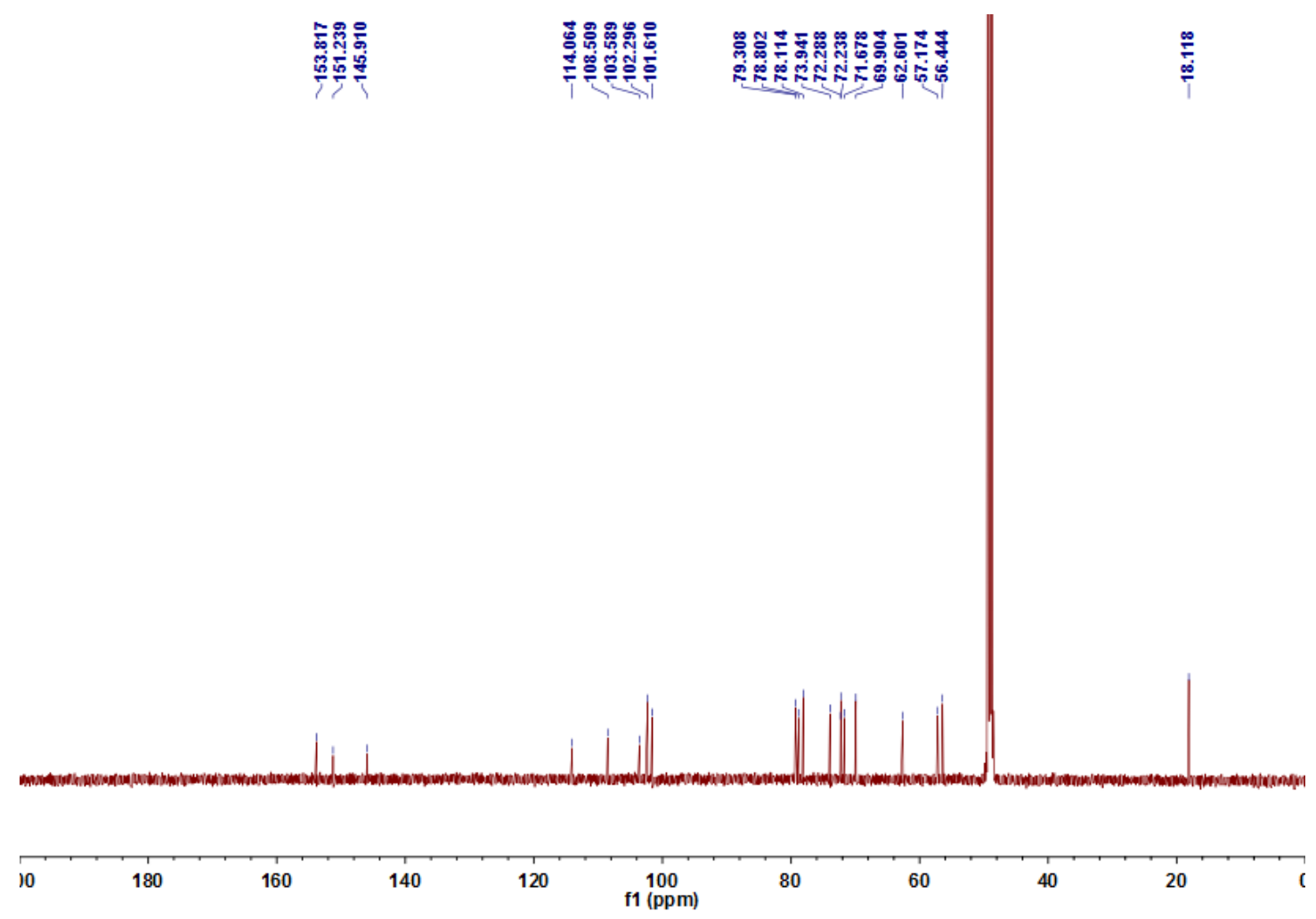

Figure S42. ${ }^{13}$ C-NMR Spectrum of Compound 6 in Methanol- $d_{4}$ 


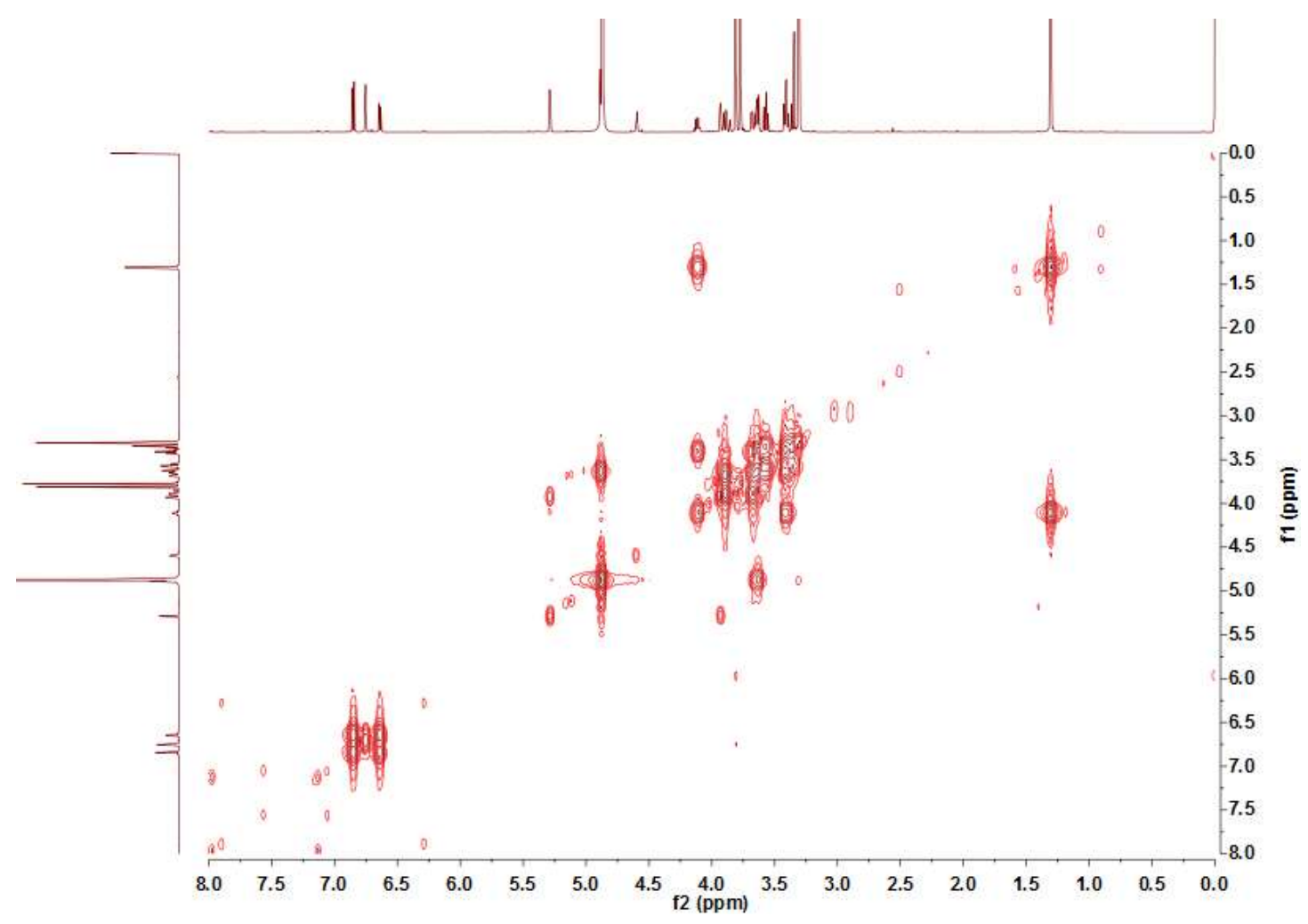

Figure S43. ${ }^{1} \mathrm{H}-{ }^{1} \mathrm{H}$ COSY Spectrum of Compound 6 in Methanol- $d_{4}$

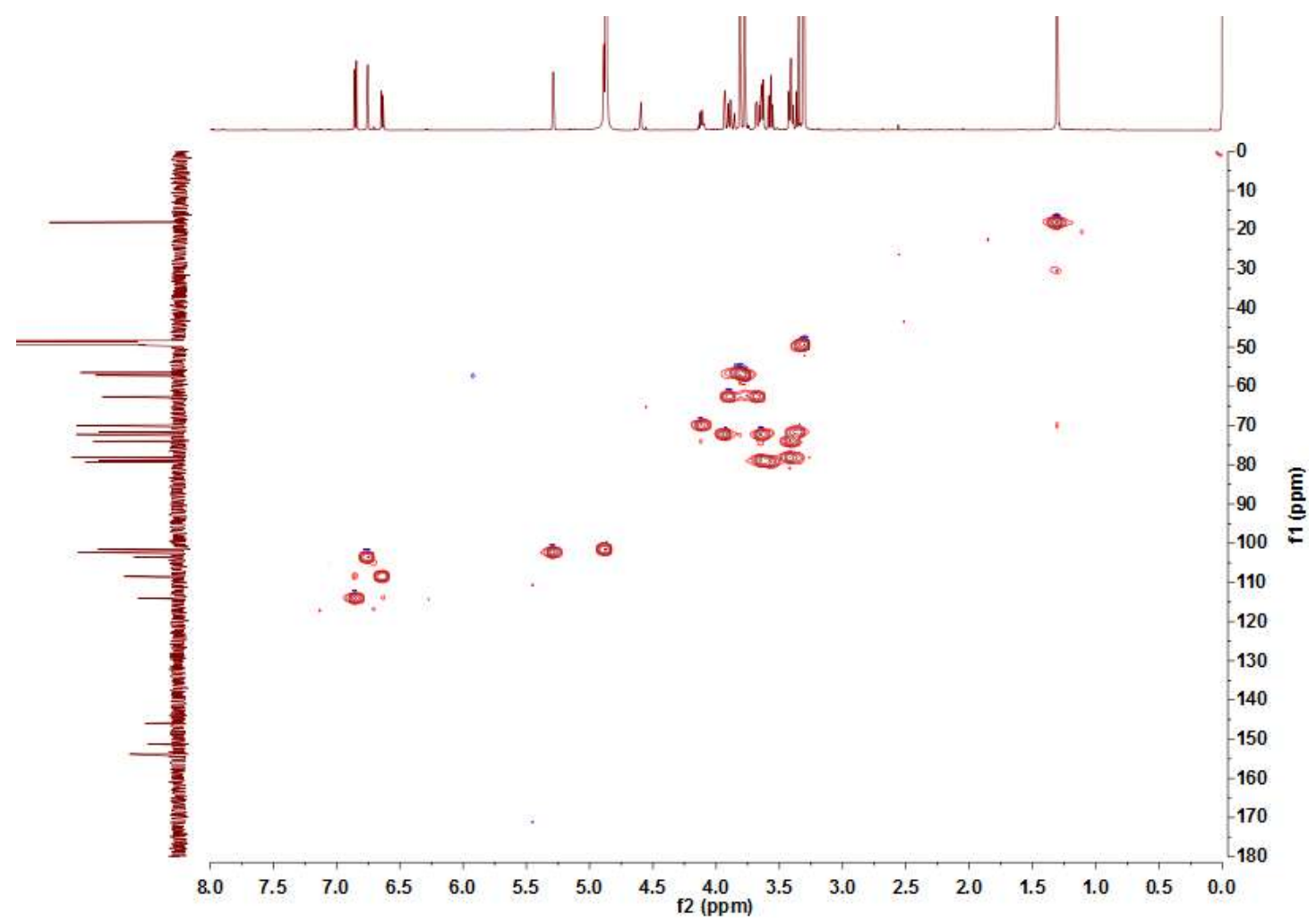

Figure S44. HSQC Spectrum of Compound 6 in Methanol- $d_{4}$ 


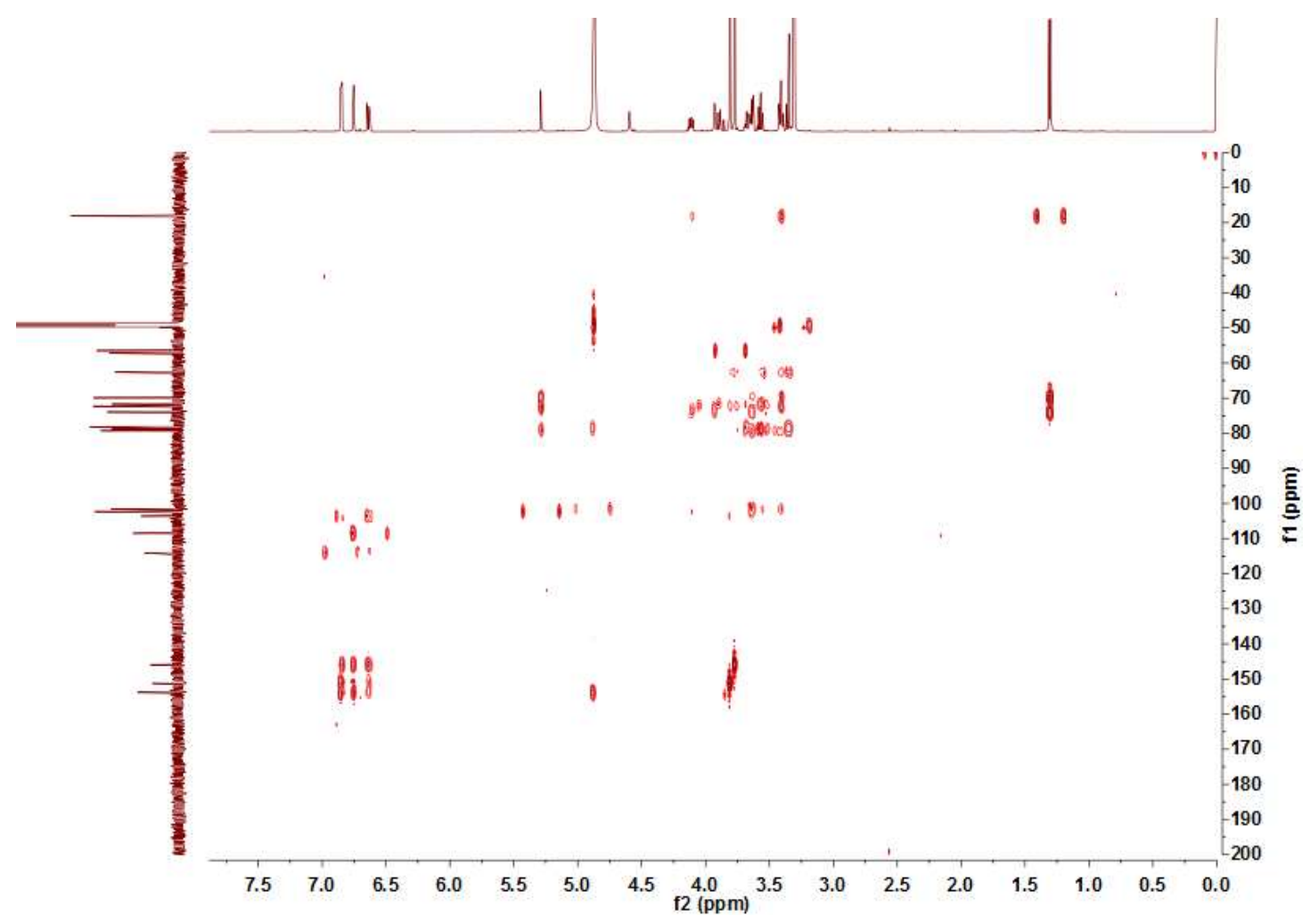

Figure S45. HMBC Spectrum of Compound 6 in Methanol- $d_{4}$

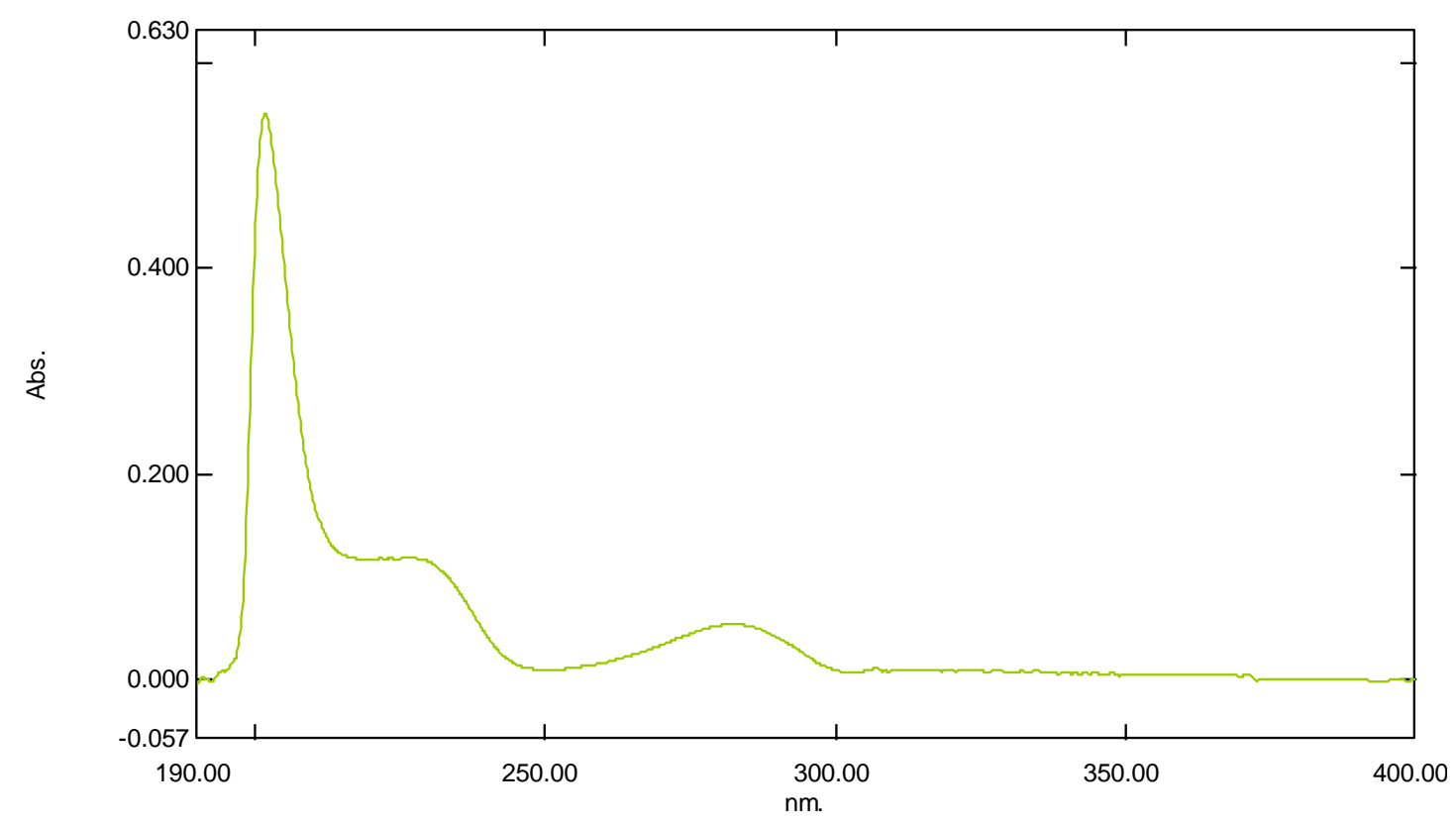

Figure S46. UV Spectrum of Compound 6 


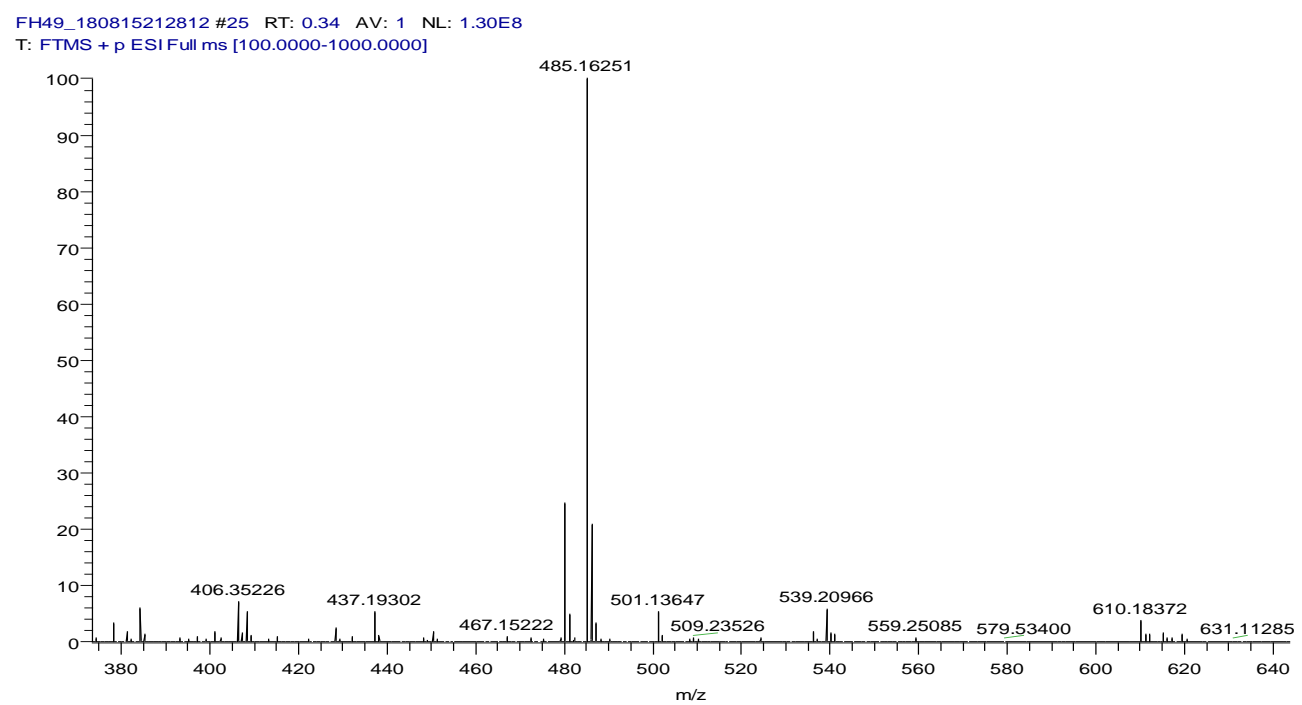

Elemental composition search on mass 485.16125

$\mathrm{m} / \mathrm{z}=480.16251-490.16000$

\begin{tabular}{c|r|r|r|l}
$\mathrm{m} / \mathrm{z}$ & Theo. Mass & \multicolumn{1}{|c|}{$\begin{array}{c}\text { Delta } \\
(\mathrm{mmu})\end{array}$} & $\begin{array}{r}\text { RDB } \\
\text { equiv. }\end{array}$ & \multicolumn{1}{c}{ Composition } \\
\hline 485.16251 & 485.16295 & -0.44 & 5.5 & $\mathrm{C}_{20} \mathrm{H}_{30} \mathrm{O}_{12} \mathrm{Na}$ \\
485.16535 & -2.84 & 8.5 & $\mathrm{C}_{22} \mathrm{H}_{29} \mathrm{O}_{12}$ \\
485.15948 & 3.03 & 17.5 & $\mathrm{C}_{29} \mathrm{H}_{25} \mathrm{O}_{7}$ \\
& 485.15707 & 5.44 & 14.5 & $\mathrm{C}_{27} \mathrm{H}_{26} \mathrm{O}_{7} \mathrm{Na}$ \\
485.15010 & 12.41 & 4.5 & $\mathrm{C}_{18} \mathrm{H}_{29} \mathrm{O}_{15}$ \\
& 485.14769 & 14.82 & 1.5 & $\mathrm{C}_{16} \mathrm{H}_{30} \mathrm{O}_{15} \mathrm{Na}$ \\
485.17820 & -15.69 & 9.5 & $\mathrm{C}_{24} \mathrm{H}_{30} \mathrm{O}_{9} \mathrm{Na}$ \\
485.18061 & -18.10 & 12.5 & $\mathrm{C}_{26} \mathrm{H}_{29} \mathrm{O}_{9}$ \\
& 485.14422 & 18.29 & 13.5 & $\mathrm{C}_{25} \mathrm{H}_{25} \mathrm{O}_{10}$ \\
& 485.14182 & 20.69 & 10.5 & $\mathrm{C}_{23} \mathrm{H}_{26} \mathrm{O}_{10} \mathrm{Na}$
\end{tabular}

Figure 47. HRESIMS Spectrum of Compound 6

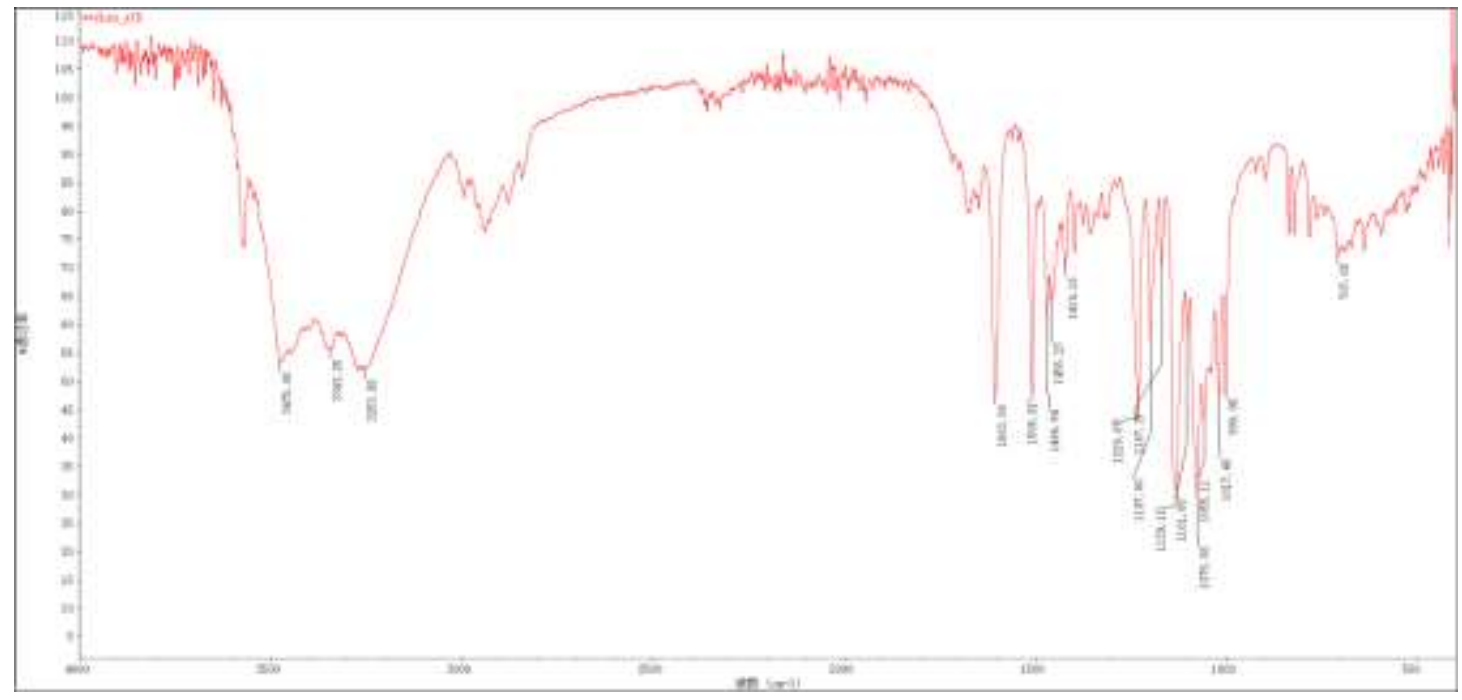

Figure 48. IR Spectrum of Compound 6 


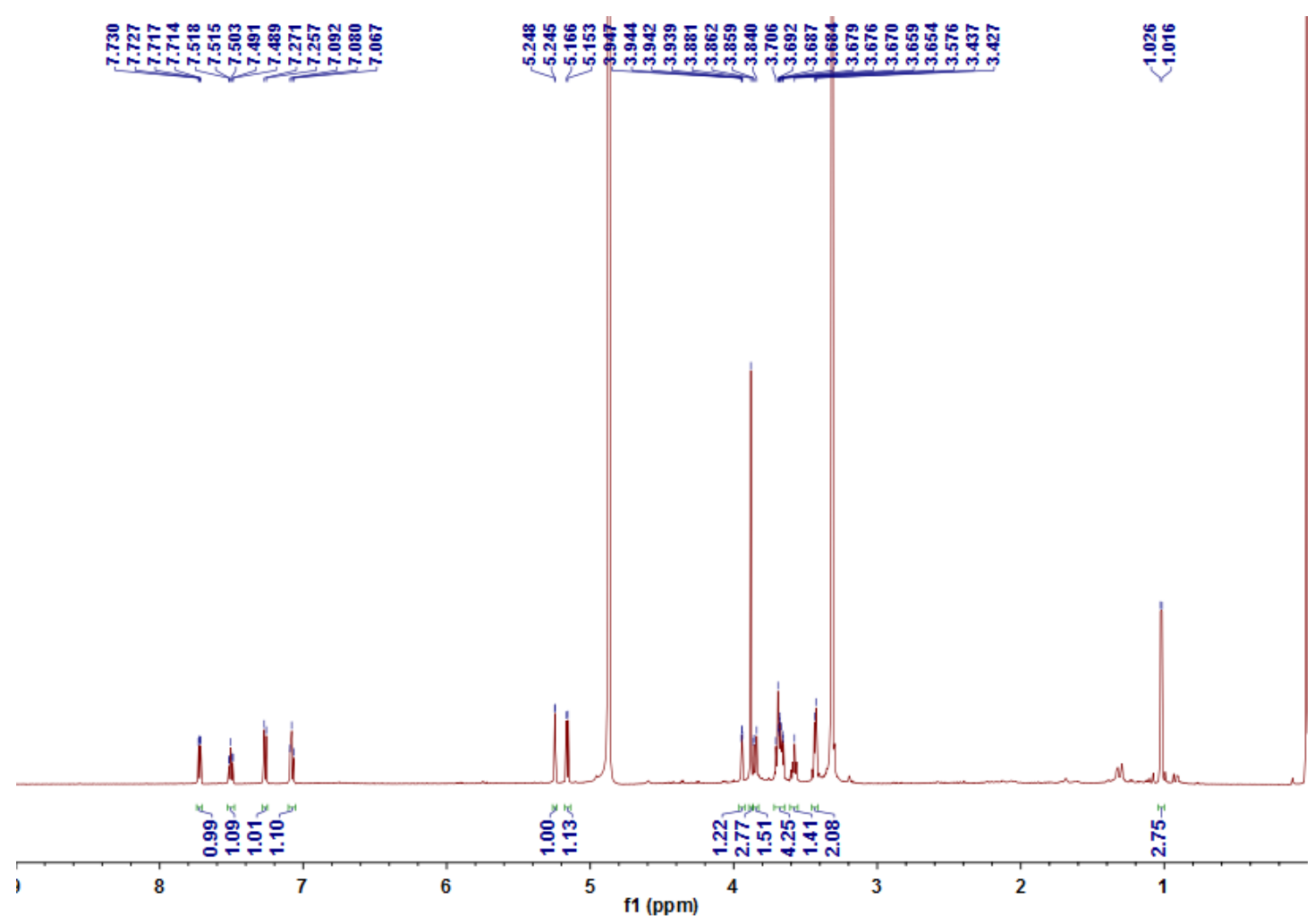

Figure S49. ${ }^{1} \mathrm{H}-\mathrm{NMR}$ Spectrum of Compound 7 in Methanol- $d_{4}$

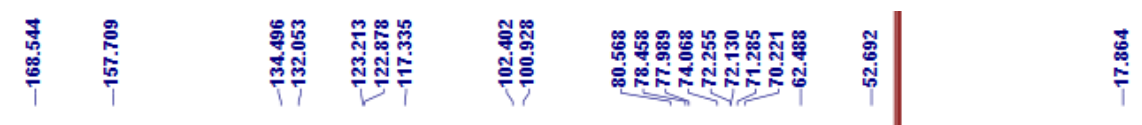

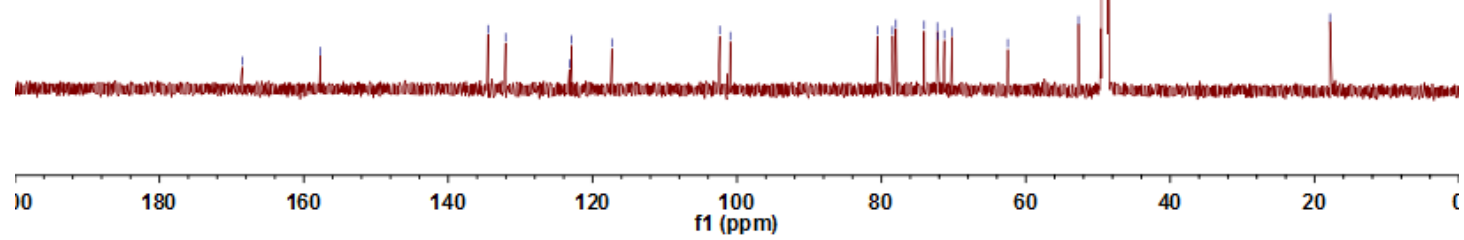

Figure S50. ${ }^{13} \mathrm{C}-\mathrm{NMR}$ Spectrum of Compound 7 in Methanol- $d_{4}$ 


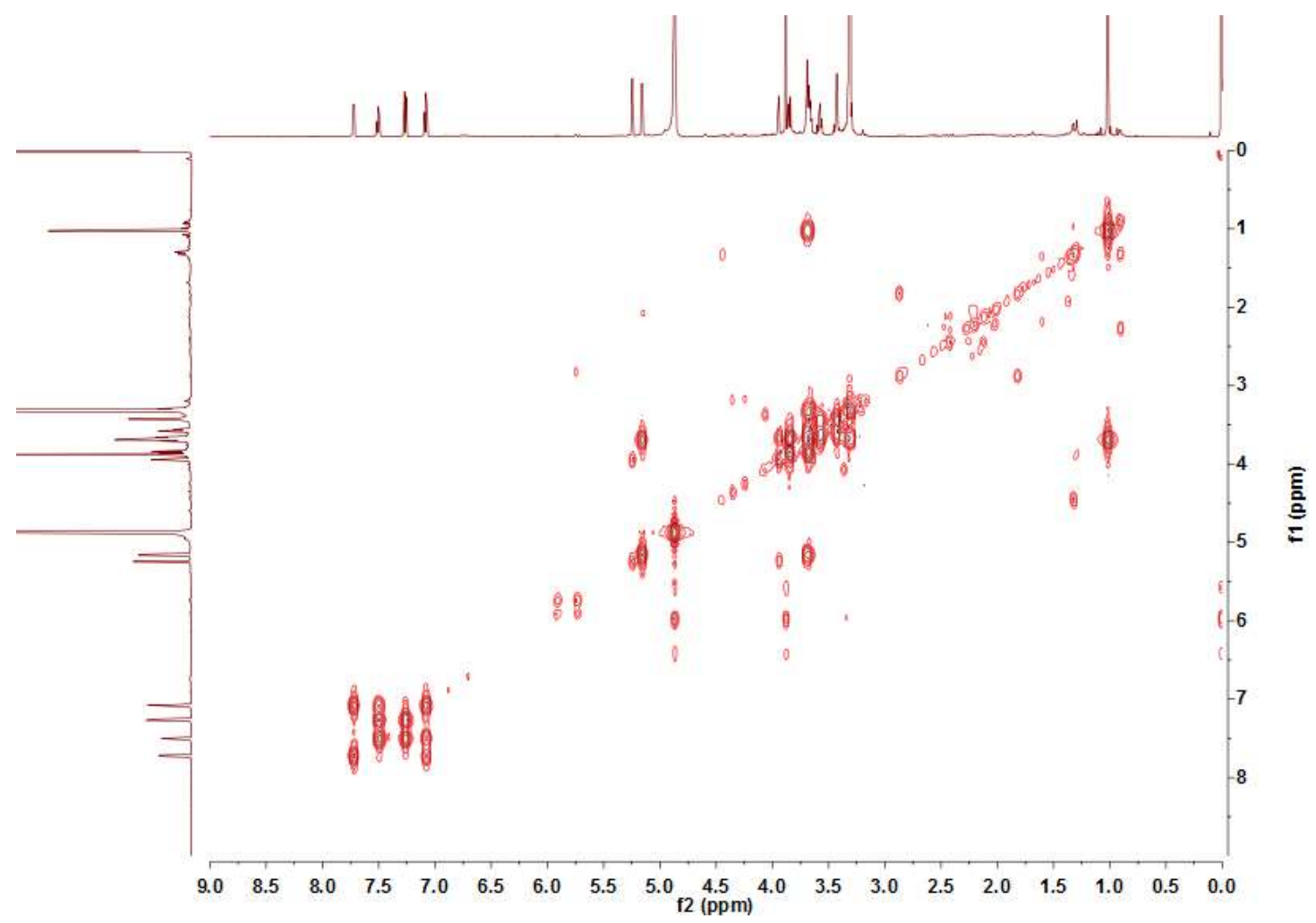

Figure S51. ${ }^{1} \mathrm{H}-{ }^{1} \mathrm{H}$ COSY Spectrum of Compound 7 in Methanol- $d_{4}$

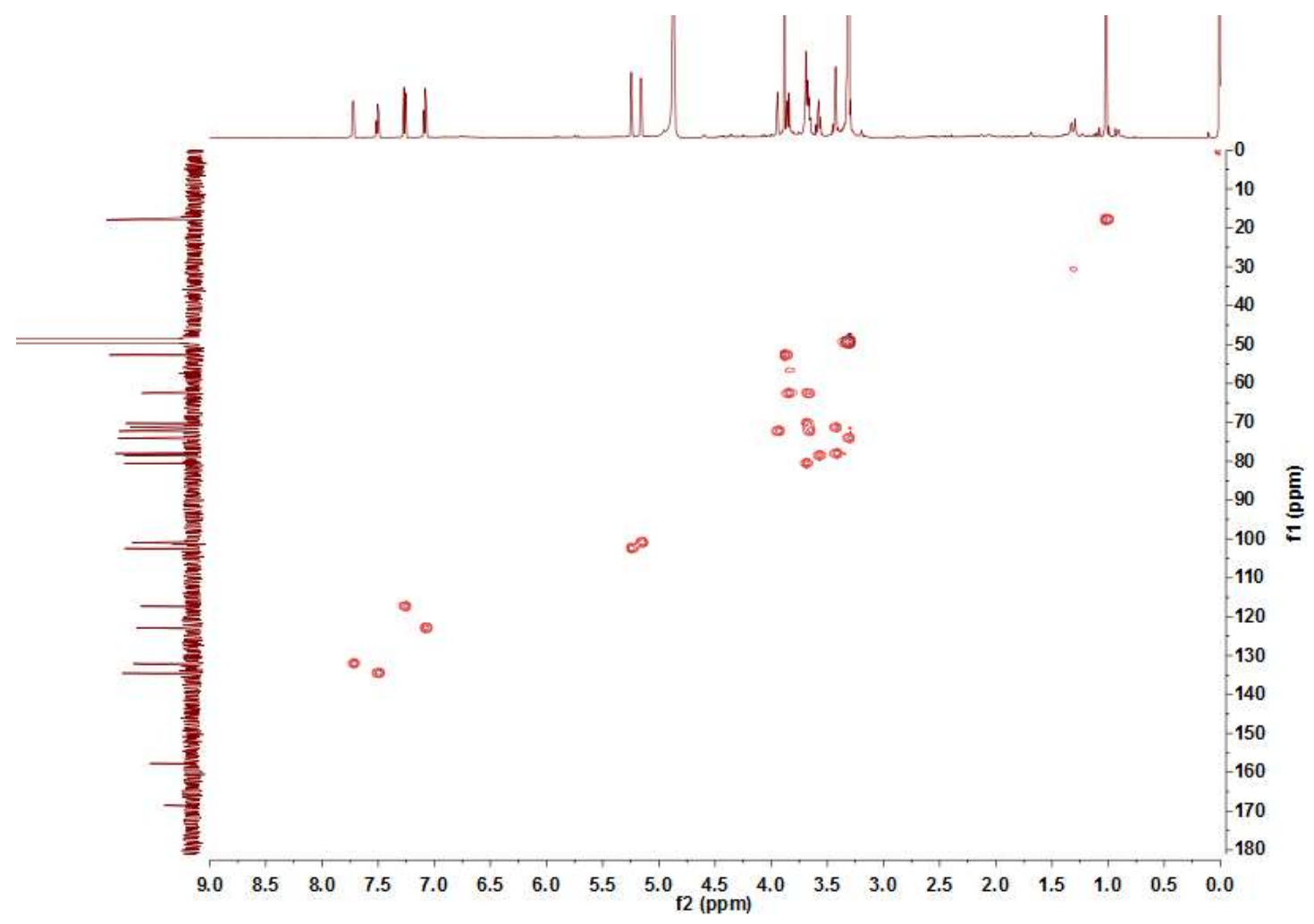

Figure S52. HSQC Spectrum of Compound 7 in Methanol- $d_{4}$ 


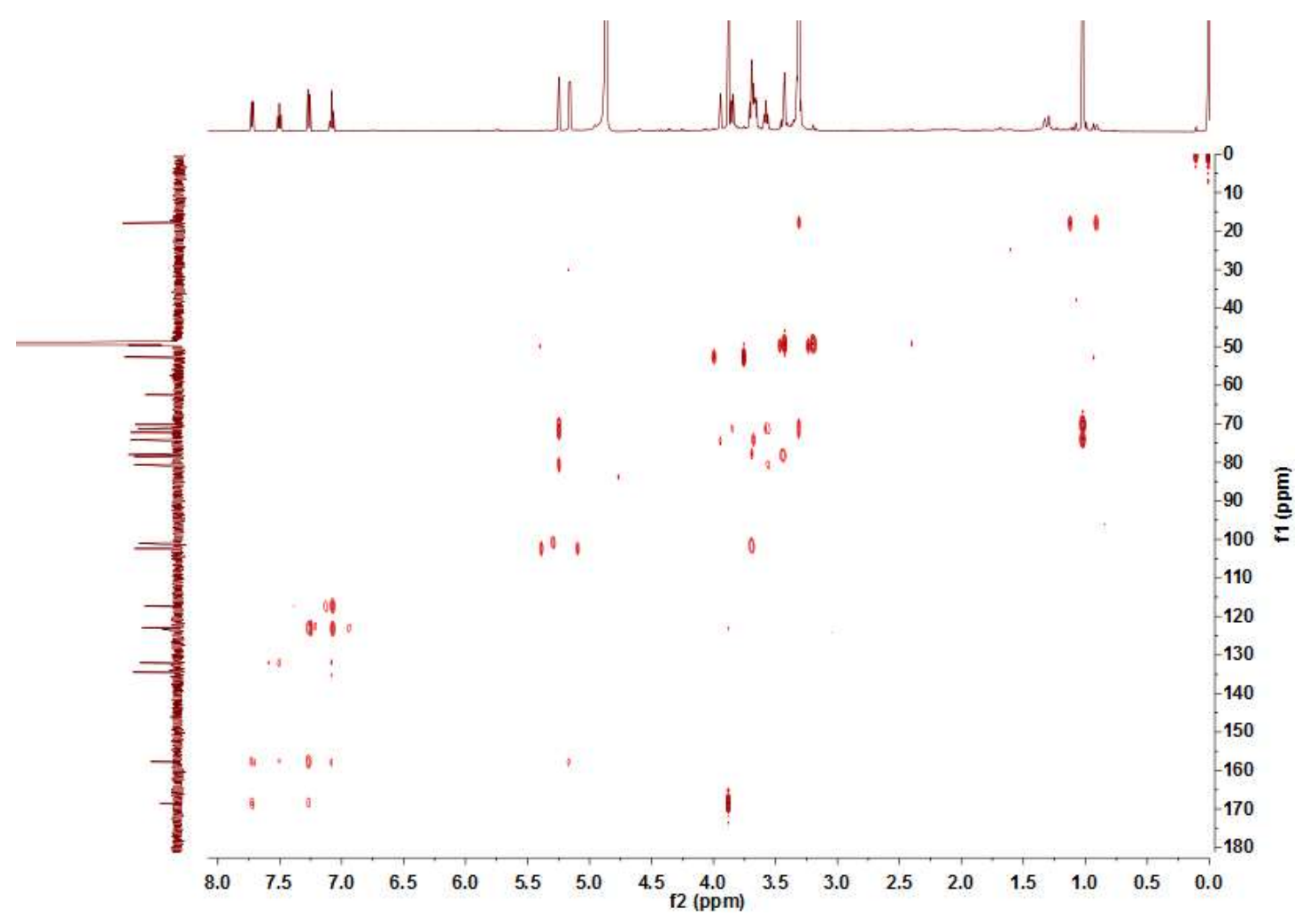

Figure S53. HMBC Spectrum of Compound 7 in Methanol- $d_{4}$

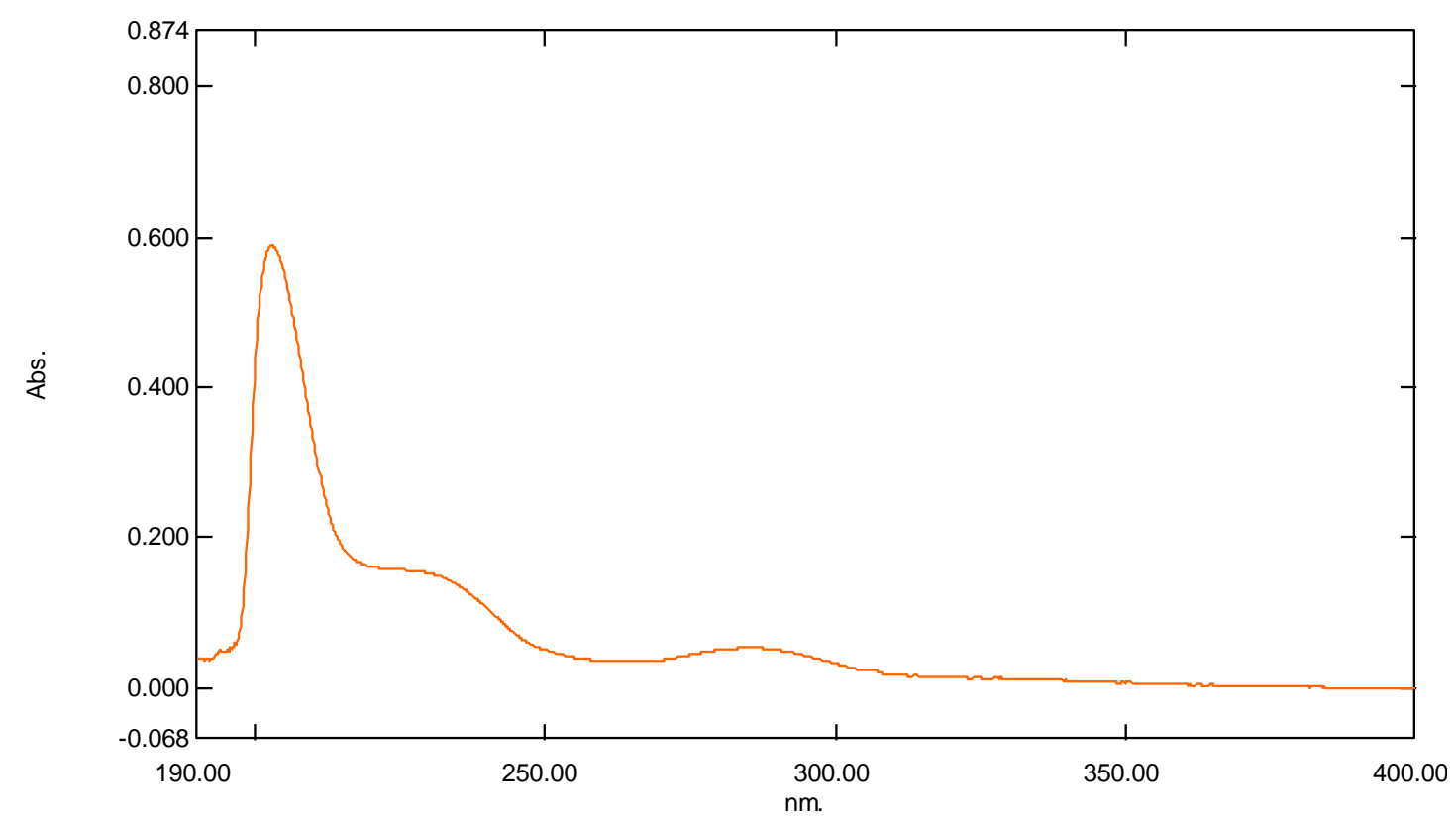

Figure S54. UV Spectrum of Compound 7 


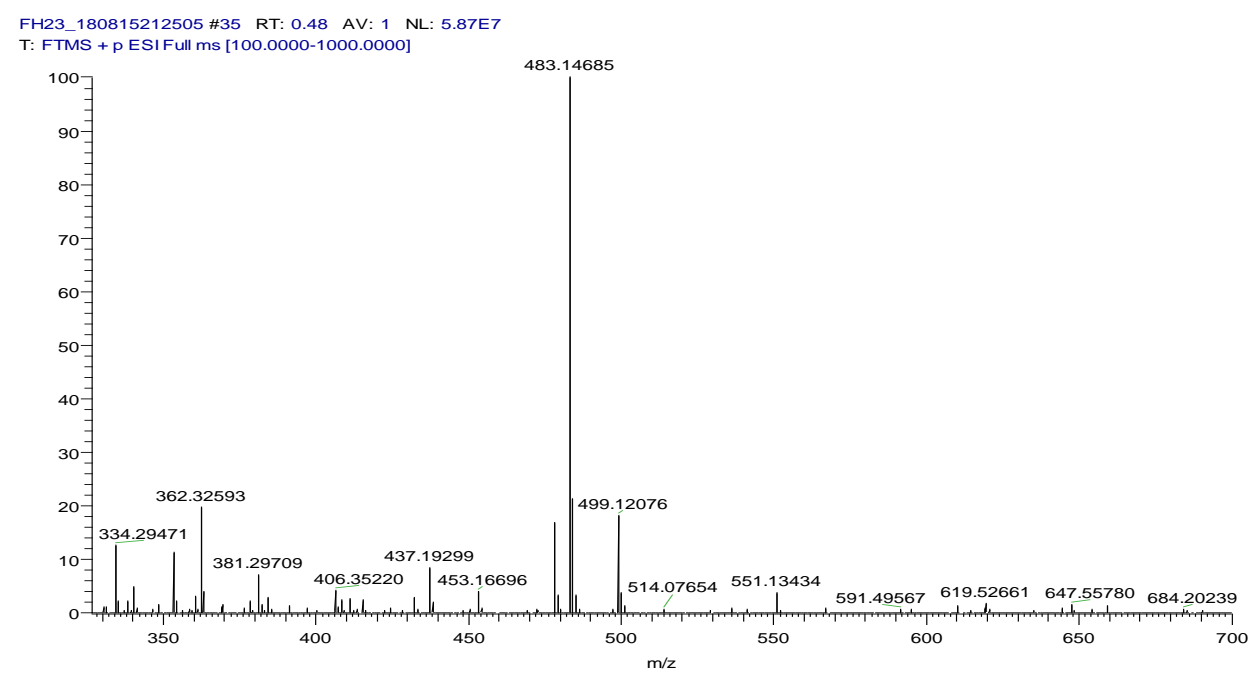

Elemental composition search on mass 483.14842

$\mathrm{m} / \mathrm{z}=478.15000-488.14685$

\begin{tabular}{|c|c|c|c|c|}
\hline $\mathrm{m} / \mathrm{z}$ & Theo. Mass & $\begin{array}{r}\text { Delta } \\
\text { (mmu) }\end{array}$ & $\begin{array}{c}\text { RDB } \\
\text { equiv. }\end{array}$ & Composition \\
\hline \multirow[t]{10}{*}{483.14685} & 483.14730 & -0.45 & 6.5 & $\mathrm{C}_{20} \mathrm{H}_{28} \mathrm{O}_{12} \mathrm{Na}$ \\
\hline & 483.14970 & -2.85 & 9.5 & $\mathrm{C}_{22} \mathrm{H}_{27} \mathrm{O}_{12}$ \\
\hline & 483.14383 & 3.02 & 18.5 & $\mathrm{C}_{29} \mathrm{H}_{23} \mathrm{O}_{7}$ \\
\hline & 483.14142 & 5.43 & 15.5 & $\mathrm{C}_{27} \mathrm{H}_{24} \mathrm{O}_{7} \mathrm{Na}$ \\
\hline & 483.13445 & 12.40 & 5.5 & $\mathrm{C}_{18} \mathrm{H}_{27} \mathrm{O}_{15}$ \\
\hline & 483.13204 & 14.81 & 2.5 & $\mathrm{C}_{16} \mathrm{H}_{28} \mathrm{O}_{15} \mathrm{Na}$ \\
\hline & 483.16255 & -15.70 & 10.5 & $\mathrm{C}_{24} \mathrm{H}_{28} \mathrm{O}_{9} \mathrm{Na}$ \\
\hline & 483.16496 & -18.11 & 13.5 & $\mathrm{C}_{26} \mathrm{H}_{27} \mathrm{O}_{9}$ \\
\hline & 483.12857 & 18.28 & 14.5 & $\mathrm{C}_{25} \mathrm{H}_{23} \mathrm{O}_{10}$ \\
\hline & 483.12617 & 20.68 & 11.5 & $\mathrm{C}_{23} \mathrm{H}_{24} \mathrm{O}_{10} \mathrm{Na}$ \\
\hline
\end{tabular}

Figure 55. HRESIMS Spectrum of Compound 7

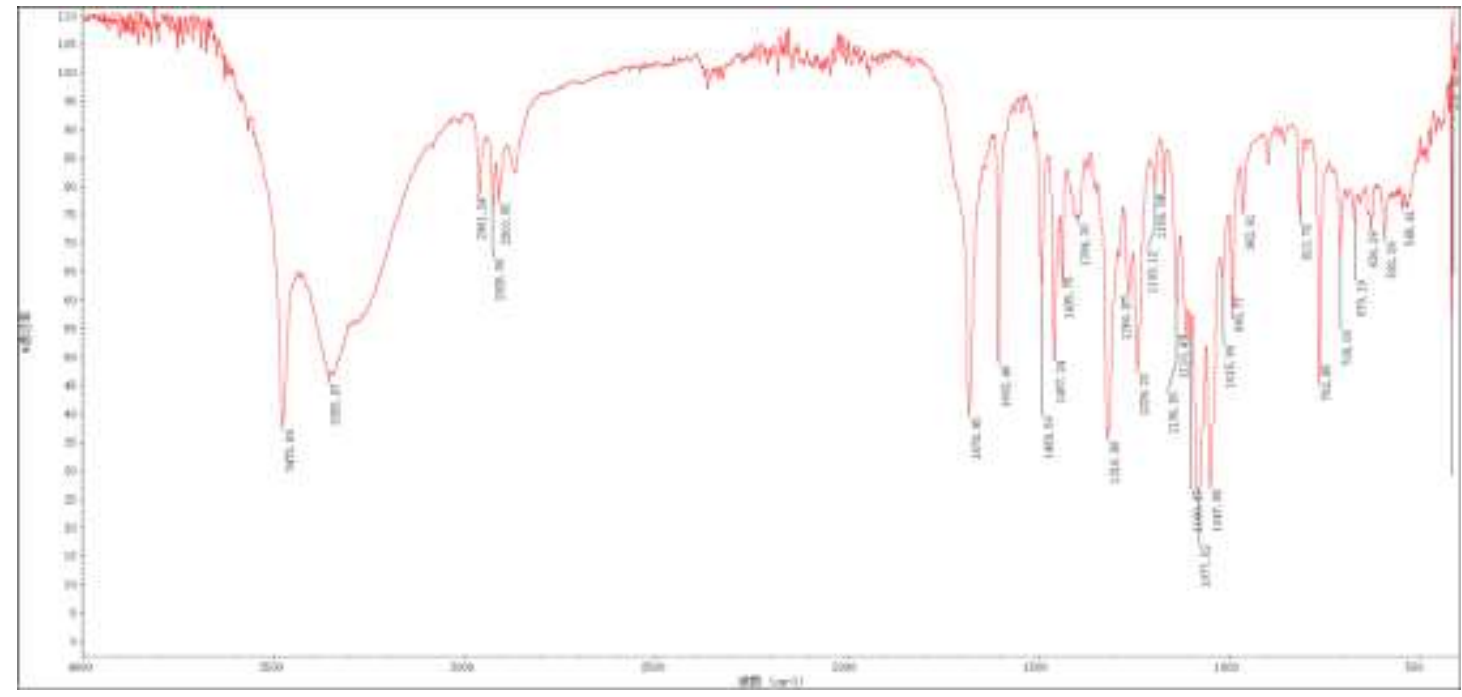

Figure 56. IR Spectrum of Compound 7 
Determination of the Absolute Configuration of Sugars. Compounds 1 (1.5 mg) and $6(1.5 \mathrm{mg})$ in $1 \mathrm{~mL}$ of $1.0 \mathrm{~N} \mathrm{HCl}$ were heated at $80^{\circ} \mathrm{C}$ for $4 \mathrm{~h}$, respectively. The reaction solution was evaporated and then dissolved by anhydrous pyridine $(0.3 \mathrm{~mL})$. The mixture was heated with L-cysteine methyl ester hydrochloride $(2.5 \mathrm{mg})$ and successively with $o$-tolyl isothiocyanate $(150 \mu \mathrm{L})$ at $60^{\circ} \mathrm{C}$ for each $1 \mathrm{~h}$. The retention times of the monosaccharide derivatives were detected at 16.85 (L-glucose), 18.31 (D-glucose), 29.39 (D-apiose), 16.69 (D-rhamnose) and 30.81 (L-rhamnose) min by HPLC, respectively.

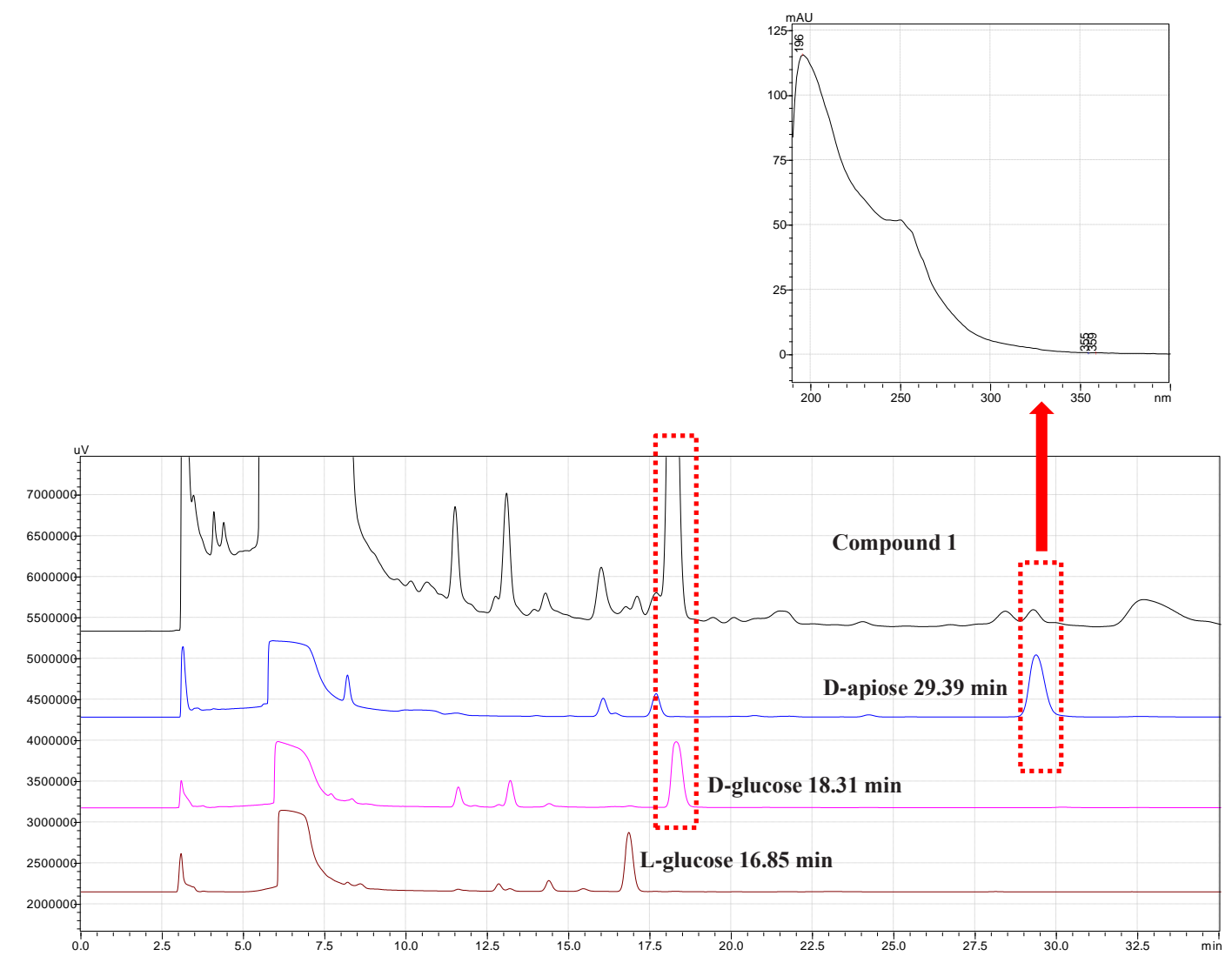

Figure S57. HPLC analysis for derivatives of D-glucose, L-glucose, D-apiose and hydrolysates of compound 1 . 


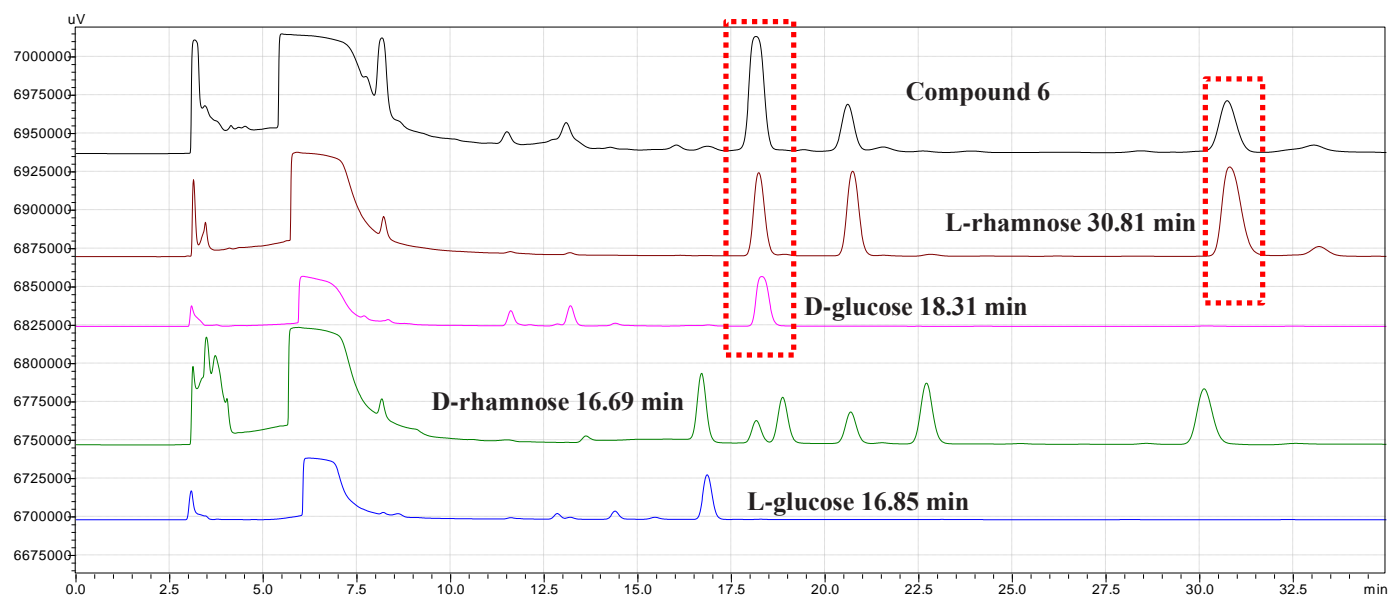

Figure S58. HPLC analysis for derivatives of D-glucose, L-glucose, D-rhamnose, L-rhamnose and hydrolysates of compound $\mathbf{6}$.

\section{ECD calculation details of 1 in $\mathrm{MeOH}$}

Monte Carlo conformational searches were carried out by means of the Spartan's 10 software using Merck Molecular Force Field (MMFF). The conformers with Boltzmann-population of over 5\% were chosen for ECD calculations, and then the conformers were initially optimized at B3LYP/6-31g (d, p) level in $\mathrm{MeOH}$ using the CPCM polarizable conductor calculation model. The theoretical calculation of ECD was conducted in $\mathrm{MeOH}$ using Time-dependent Density functional theory (TD-DFT) at the B3LYP/6-31+g (d, p) level for all conformers of compound 1. Rotatory strengths for a total of 30 excited states were calculated. ECD spectra were generated using the program SpecDis 1.6 (University of Würzburg, Würzburg, Germany) and GraphPad Prism 5 (University of California San Diego, USA) from dipole-length rotational strengths by applying Gaussian band shapes with sigma $=0.3 \mathrm{eV}$.

Table S1. Gibbs free energies ${ }^{a}$ and equilibrium populations ${ }^{b}$ of low-energy conformers of 1 .

\begin{tabular}{|c|c|c|}
\hline \multirow{2}{*}{ Conformers } & \multicolumn{2}{|c|}{ In $\mathrm{MeOH}$} \\
\cline { 2 - 3 } & $\Delta G$ & $P(\%) / 100$ \\
\hline $\mathbf{1 R - 1}$ & 0.00 & 0.415 \\
\hline $\mathbf{1 R - 2}$ & 0.52 & 0.174 \\
\hline $\mathbf{1 R - 3}$ & 0.62 & 0.145 \\
\hline $\mathbf{1 R - 4}$ & 1.09 & 0.066 \\
\hline
\end{tabular}




\begin{tabular}{|c|c|c|}
\hline 1R-5 & 1.10 & 0.065 \\
\hline & & \\
\hline
\end{tabular}

${ }^{a} \mathrm{~B} 3 \mathrm{LYP} / 6-31+\mathrm{G}(\mathrm{d}, \mathrm{p})$, in $\mathrm{kcal} / \mathrm{mol} .{ }^{b}$ From $\Delta G$ values at $298.15 \mathrm{~K}$.

Table S2. Cartesian coordinates for the low-energy reoptimized MMFF conformers of 1 at

B3LYP/6-311+G(d,p) level of theory in $\mathrm{CH}_{3} \mathrm{OH}$.

\begin{tabular}{|c|c|c|c|c|c|}
\hline \multicolumn{2}{|c|}{ 1R-1 } & \multicolumn{4}{|c|}{$\begin{array}{c}\text { Standard Orientation } \\
\text { (Ångstroms) }\end{array}$} \\
\hline $\begin{array}{l}\text { Center } \\
\text { number }\end{array}$ & $\begin{array}{l}\text { Atomic } \\
\text { number }\end{array}$ & $\begin{array}{l}\text { Atomic } \\
\text { Type }\end{array}$ & $X$ & $\mathrm{Y}$ & $\mathrm{Z}$ \\
\hline 1. & 8. & 0. & -0.256499 & 0.104125 & 0.576128 \\
\hline 2. & 6. & 0. & -2.529670 & 0.322745 & 1.377780 \\
\hline 3. & 6. & 0. & -3.856565 & -0.114727 & 1.369452 \\
\hline 4. & 6. & 0. & -4.249273 & -1.159872 & 0.531967 \\
\hline 5. & 6. & 0. & -3.292684 & -1.783692 & -0.283707 \\
\hline 6. & 6. & 0. & -1.966988 & -1.352730 & -0.278676 \\
\hline 7. & 6. & 0. & -1.587930 & -0.278385 & 0.550911 \\
\hline 8. & 8. & 0. & -0.966984 & -1.915019 & -1.032390 \\
\hline 9. & 6. & 0. & -1.313407 & -2.943671 & -1.959413 \\
\hline 10. & 6. & 0. & -5.694845 & -1.632479 & 0.478691 \\
\hline 11. & 6. & 0. & -6.478578 & -1.056457 & -0.710793 \\
\hline 12. & 6. & 0. & -6.677805 & 0.459357 & -0.660605 \\
\hline 13. & 8. & 0. & -5.776635 & -3.052935 & 0.340528 \\
\hline 14. & 6. & 0. & 1.559189 & 3.759628 & -0.031469 \\
\hline 15. & 6. & 0. & 2.108482 & 2.604722 & -0.869268 \\
\hline 16. & 6. & 0. & 1.588396 & 1.253628 & -0.369465 \\
\hline 17. & 6. & 0. & 0.074456 & 1.273449 & -0.139461 \\
\hline 18. & 8. & 0. & -0.300713 & 2.392146 & 0.644525 \\
\hline 19. & 6. & 0. & 0.036542 & 3.658686 & 0.044575 \\
\hline 20. & 6. & 0. & -0.620458 & 4.723434 & 0.911093 \\
\hline 21. & 8. & 0. & -2.030181 & 4.583157 & 0.922898 \\
\hline 22. & 8. & 0. & 1.909758 & 5.007513 & -0.603947 \\
\hline 23. & 8. & 0. & 3.520704 & 2.690901 & -0.812471 \\
\hline 24. & 8. & 0. & 1.861907 & 0.262255 & -1.366017 \\
\hline 25. & 6. & 0. & 3.916101 & -2.193341 & 0.426074 \\
\hline 26. & 6. & 0. & 2.517639 & -1.638911 & 0.134258 \\
\hline 27. & 6. & 0. & 2.793083 & -0.735953 & -1.080671 \\
\hline 28. & 8. & 0. & 4.075152 & -0.158656 & -0.824892 \\
\hline 29. & 6. & 0. & 4.816773 & -0.992345 & 0.093785 \\
\hline 30. & 8. & 0. & 4.209465 & -3.234412 & -0.517303 \\
\hline 31. & 8. & 0. & 1.626438 & -2.696424 & -0.137941 \\
\hline 32. & 8. & 0. & 5.422391 & -3.285624 & 1.970170 \\
\hline
\end{tabular}




\begin{tabular}{|c|c|c|c|c|c|}
\hline 33. & 6. & 0. & 4.117082 & -2.754335 & 1.834371 \\
\hline 34. & 1. & 0. & -2.200066 & 1.119483 & 2.034437 \\
\hline 35. & 1. & 0. & -4.582643 & 0.358344 & 2.024256 \\
\hline 36. & 1. & 0. & -3.608771 & -2.615775 & -0.900959 \\
\hline 37. & 1. & 0. & -0.380969 & -3.216967 & -2.453542 \\
\hline 38. & 1. & 0. & -2.032215 & -2.577851 & -2.700248 \\
\hline 39. & 1. & 0. & -1.727163 & -3.820929 & -1.449471 \\
\hline 40. & 1. & 0. & -6.195135 & -1.311785 & 1.407479 \\
\hline 41. & 1. & 0. & -5.959588 & -1.343037 & -1.633430 \\
\hline 42. & 1. & 0. & -7.449136 & -1.565229 & -0.725130 \\
\hline 43. & 1. & 0. & -7.216349 & 0.762126 & 0.244651 \\
\hline 44. & 1. & 0. & -5.723560 & 0.994470 & -0.675099 \\
\hline 45. & 1. & 0. & -7.263373 & 0.798734 & -1.520109 \\
\hline 46. & 1. & 0. & -5.287731 & -3.447010 & 1.075787 \\
\hline 47. & 1. & 0. & 1.964466 & 3.665718 & 0.989532 \\
\hline 48. & 1. & 0. & 1.750935 & 2.738585 & -1.904260 \\
\hline 49. & 1. & 0. & 2.074974 & 1.023066 & 0.583613 \\
\hline 50. & 1. & 0. & -0.455740 & 1.291489 & -1.104876 \\
\hline 51. & 1. & 0. & -0.391895 & 3.714399 & -0.968324 \\
\hline 52. & 1. & 0. & -0.195914 & 4.666532 & 1.926567 \\
\hline 53. & 1. & 0. & -0.389465 & 5.709077 & 0.501171 \\
\hline 54. & 1. & 0. & -2.203529 & 3.658013 & 1.147119 \\
\hline 55. & 1. & 0. & 2.860099 & 4.960709 & -0.785114 \\
\hline 56. & 1. & 0. & 3.894925 & 1.824485 & -1.052508 \\
\hline 57. & 1. & 0. & 2.171370 & -1.035548 & 0.983532 \\
\hline 58. & 1. & 0. & 2.822006 & -1.350550 & -1.988277 \\
\hline 59. & 1. & 0. & 5.744926 & -1.322972 & -0.378259 \\
\hline 60. & 1. & 0. & 5.057026 & -0.403720 & 0.985463 \\
\hline 61. & 1. & 0. & 3.372470 & -3.712813 & -0.642287 \\
\hline 62. & 1. & 0. & 0.744773 & -2.316876 & -0.314782 \\
\hline 63. & 1. & 0. & 5.544416 & -3.851297 & 1.191669 \\
\hline 64. & 1. & 0. & 4.004796 & -1.968028 & 2.588628 \\
\hline 65. & 1. & 0. & 3.340409 & -3.513563 & 2.021909 \\
\hline
\end{tabular}

\begin{tabular}{|l|l|l|c|c|c|}
\hline \multicolumn{2}{|c|}{ 1R-2 } & \multicolumn{4}{c|}{$\begin{array}{c}\text { Standard Orientation } \\
\text { (Ångstroms) }\end{array}$} \\
\hline $\begin{array}{c}\text { Center } \\
\text { number }\end{array}$ & $\begin{array}{c}\text { Atom } \\
\text { number }\end{array}$ & Type & X & Y & $\mathrm{Z}$ \\
\hline 1. & 8. & 0. & -0.248233 & 0.088246 & 0.572224 \\
\hline 2. & 6. & 0. & -2.521554 & 0.269485 & 1.382095 \\
\hline 3. & 6. & 0. & -3.842635 & -0.183942 & 1.372739 \\
\hline 4. & 6. & 0. & -4.225817 & -1.221022 & 0.520935 \\
\hline 5. & 6. & 0. & -3.266689 & -1.822890 & -0.305723 \\
\hline
\end{tabular}




\begin{tabular}{|c|c|c|c|c|c|}
\hline 6. & 6. & 0. & -1.944632 & -1.380778 & -0.292858 \\
\hline 7. & 6. & 0. & -1.575625 & -0.311114 & 0.545745 \\
\hline 8. & 8. & 0. & -0.938427 & -1.929189 & -1.049949 \\
\hline 9. & 6. & 0. & -1.272996 & -2.968439 & -1.969200 \\
\hline 10. & 6. & 0. & -5.667872 & -1.688025 & 0.460013 \\
\hline 11. & 6. & 0. & -6.478231 & -0.989520 & -0.652315 \\
\hline 12. & 6. & 0. & -6.662260 & 0.518377 & -0.467674 \\
\hline 13. & 8. & 0. & -5.650863 & -3.100491 & 0.244203 \\
\hline 14. & 6. & 0. & 1.515247 & 3.767628 & -0.040092 \\
\hline 15. & 6. & 0. & 2.084064 & 2.618929 & -0.873262 \\
\hline 16. & 6. & 0. & 1.581490 & 1.261287 & -0.373674 \\
\hline 17. & 6. & 0. & 0.066982 & 1.258828 & -0.146657 \\
\hline 18. & 8. & 0. & -0.326269 & 2.375072 & 0.632873 \\
\hline 19. & 6. & 0. & -0.006000 & 3.644007 & 0.029979 \\
\hline 20. & 6. & 0. & -0.683649 & 4.700847 & 0.890368 \\
\hline 21. & 8. & 0. & -2.090521 & 4.533041 & 0.901033 \\
\hline 22. & 8. & 0. & 1.850171 & 5.019355 & -0.614098 \\
\hline 23. & 8. & 0. & 3.494996 & 2.724222 & -0.811873 \\
\hline 24. & 8. & 0. & 1.872797 & 0.274448 & -1.369654 \\
\hline 25. & 6. & 0. & 3.935413 & -2.159348 & 0.441729 \\
\hline 26. & 6. & 0. & 2.533675 & -1.622151 & 0.133847 \\
\hline 27. & 6. & 0. & 2.811503 & -0.714717 & -1.077229 \\
\hline 28. & 8. & 0. & 4.085091 & -0.123879 & -0.809153 \\
\hline 29. & 6. & 0. & 4.825081 & -0.947569 & 0.119676 \\
\hline 30. & 8. & 0. & 4.252017 & -3.197110 & -0.497910 \\
\hline 31. & 8. & 0. & 1.659484 & -2.690549 & -0.149916 \\
\hline 32. & 8. & 0. & 5.436925 & -3.233972 & 2.002798 \\
\hline 33. & 6. & 0. & 4.127004 & -2.717643 & 1.852399 \\
\hline 34. & 1. & 0. & -2.199816 & 1.061785 & 2.047948 \\
\hline 35. & 1. & 0. & -4.569873 & 0.268802 & 2.040034 \\
\hline 36. & 1. & 0. & -3.572954 & -2.653808 & -0.927508 \\
\hline 37. & 1. & 0. & -0.336469 & -3.238001 & -2.457817 \\
\hline 38. & 1. & 0. & -1.991604 & -2.614618 & -2.716280 \\
\hline 39. & 1. & 0. & -1.682225 & -3.843957 & -1.452949 \\
\hline 40. & 1. & 0. & -6.142236 & -1.458234 & 1.428575 \\
\hline 41. & 1. & 0. & -5.991997 & -1.201567 & -1.612138 \\
\hline 42. & 1. & 0. & -7.468137 & -1.468470 & -0.688965 \\
\hline 43. & 1. & 0. & -7.156456 & 0.747040 & 0.483436 \\
\hline 44. & 1. & 0. & -5.703652 & 1.044450 & -0.480418 \\
\hline 45. & 1. & 0. & -7.281864 & 0.932096 & -1.268748 \\
\hline 46. & 1. & 0. & -6.562505 & -3.382892 & 0.093361 \\
\hline 47. & 1. & 0. & 1.917512 & 3.682130 & 0.982794 \\
\hline 48. & 1. & 0. & 1.728666 & 2.746176 & -1.909845 \\
\hline
\end{tabular}




\begin{tabular}{|l|l|l|l|l|l|}
\hline 49. & 1. & 0. & 2.069397 & 1.038104 & 0.580478 \\
\hline 50. & 1. & 0. & -0.461368 & 1.266572 & -1.113257 \\
\hline 51. & 1. & 0. & -0.430788 & 3.689787 & -0.985039 \\
\hline 52. & 1. & 0. & -0.259714 & 4.656700 & 1.906713 \\
\hline 53. & 1. & 0. & -0.470978 & 5.688858 & 0.476212 \\
\hline 54. & 1. & 0. & -2.244839 & 3.604792 & 1.126871 \\
\hline 55. & 1. & 0. & 2.801672 & 4.985231 & -0.791922 \\
\hline 56. & 1. & 0. & 3.881038 & 1.861067 & -1.044833 \\
\hline 57. & 1. & 0. & 2.169464 & -1.024102 & 0.979239 \\
\hline 58. & 1. & 0. & 2.855357 & -1.327592 & -1.985354 \\
\hline 59. & 1. & 0. & 5.763489 & -1.266591 & -0.339970 \\
\hline 60. & 1. & 0. & 5.046179 & -0.355635 & 1.014162 \\
\hline 61. & 1. & 0. & 3.422142 & -3.685577 & -0.631493 \\
\hline 62. & 1. & 0. & 0.772154 & -2.323282 & -0.325183 \\
\hline 63. & 1. & 0. & 5.573614 & -3.798072 & 1.225582 \\
\hline 64. & 1. & 0. & 3.997319 & -1.932466 & 2.605031 \\
\hline 65. & 1. & 0. & 3.357139 & -3.485665 & 2.031595 \\
\hline
\end{tabular}

\begin{tabular}{|c|c|c|c|c|c|}
\hline \multicolumn{2}{|c|}{ 1R-3 } & \multicolumn{4}{|c|}{$\begin{array}{c}\text { Standard Orientation } \\
\text { (Ångstroms) }\end{array}$} \\
\hline $\begin{array}{l}\text { Center } \\
\text { number }\end{array}$ & $\begin{array}{l}\text { Atom } \\
\text { number }\end{array}$ & Type & $\mathrm{X}$ & $\mathrm{Y}$ & Z \\
\hline 1. & 8. & 0. & -0.244213 & 0.126806 & 0.625987 \\
\hline 2. & 6. & 0. & -2.502147 & 0.381276 & 1.463947 \\
\hline 3. & 6. & 0. & -3.832460 & -0.037253 & 1.480906 \\
\hline 4. & 6. & 0. & -4.253248 & -1.086985 & 0.659965 \\
\hline 5. & 6. & 0. & -3.322763 & -1.729901 & -0.164634 \\
\hline 6. & 6. & 0. & -1.989193 & -1.317129 & -0.181604 \\
\hline 7. & 6. & 0. & -1.581193 & -0.241363 & 0.627293 \\
\hline 8. & 8. & 0. & -1.011151 & -1.904008 & -0.946968 \\
\hline 9. & 6. & 0. & -1.387162 & -2.949153 & -1.843320 \\
\hline 10. & 6. & 0. & -5.711717 & -1.512839 & 0.665070 \\
\hline 11. & 6. & 0. & -6.634495 & -0.492243 & -0.037989 \\
\hline 12. & 6. & 0. & -6.347405 & -0.304842 & -1.529273 \\
\hline 13. & 8. & 0. & -5.802248 & -2.798644 & 0.052099 \\
\hline 14. & 6. & 0. & 1.606701 & 3.747607 & -0.074142 \\
\hline 15. & 6. & 0. & 2.121483 & 2.573633 & -0.907129 \\
\hline 16. & 6. & 0. & 1.594318 & 1.236945 & -0.377281 \\
\hline 17. & 6. & 0. & 0.086261 & 1.279712 & -0.113598 \\
\hline 18. & 8. & 0. & -0.255549 & 2.415736 & 0.662318 \\
\hline 19. & 6. & 0. & 0.085033 & 3.668235 & 0.036642 \\
\hline 20. & 6. & 0. & -0.539572 & 4.753644 & 0.901578 \\
\hline 21. & 8. & 0. & -1.950055 & 4.627723 & 0.948996 \\
\hline
\end{tabular}




\begin{tabular}{|c|c|c|c|c|c|}
\hline 22. & 8. & 0. & 1.961735 & 4.982541 & -0.671945 \\
\hline 23. & 8. & 0. & 3.535866 & 2.640790 & -0.883084 \\
\hline 24. & 8. & 0. & 1.833093 & 0.229380 & -1.366565 \\
\hline 25. & 6. & 0. & 3.888504 & -2.234106 & 0.413166 \\
\hline 26. & 6. & 0. & 2.492267 & -1.662002 & 0.145029 \\
\hline 27. & 6. & 0. & 2.754849 & -0.778807 & -1.087136 \\
\hline 28. & 8. & 0. & 4.050543 & -0.217020 & -0.865622 \\
\hline 29. & 6. & 0. & 4.800207 & -1.051999 & 0.045218 \\
\hline 30. & 8. & 0. & 4.144236 & -3.291718 & -0.522942 \\
\hline 31. & 8. & 0. & 1.580666 & -2.709487 & -0.094379 \\
\hline 32. & 8. & 0. & 5.410814 & -3.333081 & 1.936593 \\
\hline 33. & 6. & 0. & 4.111719 & -2.781060 & 1.823616 \\
\hline 34. & 1. & 0. & -2.151769 & 1.180232 & 2.106966 \\
\hline 35. & 1. & 0. & -4.538764 & 0.452212 & 2.145656 \\
\hline 36. & 1. & 0. & -3.660978 & -2.557755 & -0.772349 \\
\hline 37. & 1. & 0. & -0.466295 & -3.247757 & -2.344696 \\
\hline 38. & 1. & 0. & -2.111633 & -2.590495 & -2.582209 \\
\hline 39. & 1. & 0. & -1.806280 & -3.807233 & -1.306162 \\
\hline 40. & 1. & 0. & -6.033363 & -1.581328 & 1.717394 \\
\hline 41. & 1. & 0. & -7.672518 & -0.828606 & 0.102110 \\
\hline 42. & 1. & 0. & -6.556632 & 0.466598 & 0.488112 \\
\hline 43. & 1. & 0. & -5.347873 & 0.108853 & -1.691891 \\
\hline 44. & 1. & 0. & -6.403366 & -1.260002 & -2.059287 \\
\hline 45. & 1. & 0. & -7.072372 & 0.378608 & -1.981062 \\
\hline 46. & 1. & 0. & -6.736829 & -3.039341 & 0.016270 \\
\hline 47. & 1. & 0. & 2.033350 & 3.662368 & 0.938883 \\
\hline 48. & 1. & 0. & 1.743065 & 2.698208 & -1.935859 \\
\hline 49. & 1. & 0. & 2.099139 & 1.012108 & 0.567674 \\
\hline 50. & 1. & 0. & -0.465369 & 1.292399 & -1.066972 \\
\hline 51. & 1. & 0. & -0.364780 & 3.715239 & -0.967478 \\
\hline 52. & 1. & 0. & -0.091878 & 4.707625 & 1.907623 \\
\hline 53. & 1. & 0. & -0.308333 & 5.730524 & 0.471230 \\
\hline 54. & 1. & 0. & -2.125787 & 3.706048 & 1.185811 \\
\hline 55. & 1. & 0. & 2.906969 & 4.919120 & -0.873635 \\
\hline 56. & 1. & 0. & 3.892437 & 1.764847 & -1.115523 \\
\hline 57. & 1. & 0. & 2.173673 & -1.042604 & 0.993564 \\
\hline 58. & 1. & 0. & 2.755991 & -1.405504 & -1.986911 \\
\hline 59. & 1. & 0. & 5.711266 & -1.403731 & -0.444655 \\
\hline 60. & 1. & 0. & 5.071763 & -0.456847 & 0.923534 \\
\hline 61. & 1. & 0. & 3.295953 & -3.755690 & -0.625017 \\
\hline 62. & 1. & 0. & 0.699644 & -2.320396 & -0.255259 \\
\hline 63. & 1. & 0. & 5.505058 & -3.909501 & 1.162105 \\
\hline 64. & 1. & 0. & 4.029808 & -1.983985 & 2.570409 \\
\hline
\end{tabular}




\begin{tabular}{|l|l|l|l|l|l|}
\hline 65. & 1. & 0. & 3.326893 & -3.524584 & 2.037772 \\
\hline
\end{tabular}

\begin{tabular}{|c|c|c|c|c|c|}
\hline \multicolumn{2}{|c|}{ 1R-4 } & \multicolumn{4}{|c|}{$\begin{array}{l}\text { Standard Orientation } \\
\text { (Ångstroms) }\end{array}$} \\
\hline $\begin{array}{c}\text { Center } \\
\text { number }\end{array}$ & $\begin{array}{c}\text { Atom } \\
\text { number }\end{array}$ & Type & $\mathrm{X}$ & $\mathrm{Y}$ & Z \\
\hline 1. & 8. & 0. & -0.619989 & 0.348248 & 1.431561 \\
\hline 2. & 6. & 0. & -2.999674 & 0.250873 & 1.679477 \\
\hline 3. & 6. & 0. & -4.228066 & -0.356724 & 1.410627 \\
\hline 4. & 6. & 0. & -4.298678 & -1.437775 & 0.531068 \\
\hline 5. & 6. & 0. & -3.120638 & -1.919963 & -0.059745 \\
\hline 6. & 6. & 0. & -1.889356 & -1.325268 & 0.214337 \\
\hline 7. & 6. & 0. & -1.834327 & -0.220998 & 1.088507 \\
\hline 8. & 8. & 0. & -0.692971 & -1.750623 & -0.316994 \\
\hline 9. & 6. & 0. & -0.742909 & -2.741149 & -1.346328 \\
\hline 10. & 6. & 0. & -5.627278 & -2.096362 & 0.188555 \\
\hline 11. & 6. & 0. & -6.221753 & -1.611027 & -1.142327 \\
\hline 12. & 6. & 0. & -6.628403 & -0.136464 & -1.151077 \\
\hline 13. & 8. & 0. & -5.490756 & -3.513213 & 0.054323 \\
\hline 14. & 6. & 0. & 1.022183 & 3.833495 & -0.134466 \\
\hline 15. & 6. & 0. & 1.953570 & 2.626484 & 0.006571 \\
\hline 16. & 6. & 0. & 1.346676 & 1.608066 & 0.981314 \\
\hline 17. & 6. & 0. & -0.056916 & 1.224929 & 0.491693 \\
\hline 18. & 8. & 0. & -0.846841 & 2.403213 & 0.432407 \\
\hline 19. & 6. & 0. & -0.377850 & 3.359704 & -0.535160 \\
\hline 20. & 6. & 0. & -1.427298 & 4.462883 & -0.568238 \\
\hline 21. & 8. & 0. & -2.695036 & 3.952601 & -0.938395 \\
\hline 22. & 8. & 0. & 1.504719 & 4.721093 & -1.125568 \\
\hline 23. & 8. & 0. & 3.215298 & 3.107828 & 0.427885 \\
\hline 24. & 8. & 0. & 2.176157 & 0.473036 & 1.235601 \\
\hline 25. & 6. & 0. & 4.359028 & -1.943416 & -0.193983 \\
\hline 26. & 6. & 0. & 3.072050 & -1.625390 & 0.576510 \\
\hline 27. & 6. & 0. & 2.758343 & -0.186563 & 0.150448 \\
\hline 28. & 8. & 0. & 4.007043 & 0.408093 & -0.215013 \\
\hline 29. & 6. & 0. & 5.057255 & -0.580018 & -0.195999 \\
\hline 30. & 8. & 0. & 4.027122 & -2.267947 & -1.549101 \\
\hline 31. & 8. & 0. & 2.059525 & -2.554974 & 0.240368 \\
\hline 32. & 8. & 0. & 6.336302 & -3.314804 & -0.432689 \\
\hline 33. & 6. & 0. & 5.203636 & -3.080822 & 0.383453 \\
\hline 34. & 1. & 0. & -2.919065 & 1.091993 & 2.359361 \\
\hline 35. & 1. & 0. & -5.129939 & 0.015756 & 1.887726 \\
\hline 36. & 1. & 0. & -3.193684 & -2.779468 & -0.714606 \\
\hline 37. & 1. & 0. & 0.288804 & -2.881893 & -1.665352 \\
\hline
\end{tabular}




\begin{tabular}{|c|c|c|c|c|c|}
\hline 38. & 1. & 0. & -1.354549 & -2.401133 & -2.188881 \\
\hline 39. & 1. & 0. & -1.137488 & -3.690963 & -0.968664 \\
\hline 40. & 1. & 0. & -6.344355 & -1.859083 & 0.991975 \\
\hline 41. & 1. & 0. & -5.491932 & -1.810485 & -1.936245 \\
\hline 42. & 1. & 0. & -7.092000 & -2.244377 & -1.349186 \\
\hline 43. & 1. & 0. & -7.374274 & 0.076972 & -0.376819 \\
\hline 44. & 1. & 0. & -5.771443 & 0.521674 & -0.978888 \\
\hline 45. & 1. & 0. & -7.068292 & 0.135513 & -2.115053 \\
\hline 46. & 1. & 0. & -5.107195 & -3.848548 & 0.876134 \\
\hline 47. & 1. & 0. & 0.959344 & 4.332409 & 0.847123 \\
\hline 48. & 1. & 0. & 2.039309 & 2.167362 & -0.992173 \\
\hline 49. & 1. & 0. & 1.222587 & 2.075820 & 1.962278 \\
\hline 50. & 1. & 0. & -0.033894 & 0.754756 & -0.504601 \\
\hline 51. & 1. & 0. & -0.329040 & 2.887660 & -1.529178 \\
\hline 52. & 1. & 0. & -1.457153 & 4.953881 & 0.418190 \\
\hline 53. & 1. & 0. & -1.139104 & 5.210190 & -1.311159 \\
\hline 54. & 1. & 0. & -2.859094 & 3.201585 & -0.349739 \\
\hline 55. & 1. & 0. & 2.449656 & 4.829695 & -0.938582 \\
\hline 56. & 1. & 0. & 3.835385 & 2.365397 & 0.322364 \\
\hline 57. & 1. & 0. & 3.267117 & -1.617468 & 1.658499 \\
\hline 58. & 1. & 0. & 2.101526 & -0.177472 & -0.729029 \\
\hline 59. & 1. & 0. & 5.681022 & -0.449998 & -1.082006 \\
\hline 60. & 1. & 0. & 5.674131 & -0.450369 & 0.701746 \\
\hline 61. & 1. & 0. & 3.203425 & -2.780319 & -1.493204 \\
\hline 62. & 1. & 0. & 1.191682 & -2.177699 & 0.468293 \\
\hline 63. & 1. & 0. & 5.987886 & -3.372879 & -1.336078 \\
\hline 64. & 1. & 0. & 5.569065 & -2.828067 & 1.384439 \\
\hline 65. & 1. & 0. & 4.569409 & -3.977814 & 0.474817 \\
\hline
\end{tabular}

\begin{tabular}{|l|l|l|l|l|l|}
\hline \multicolumn{2}{|c|}{1 1R-5 } & \multicolumn{4}{c|}{$\begin{array}{c}\text { Standard Orientation } \\
\text { (Angstroms) }\end{array}$} \\
\hline $\begin{array}{c}\text { Center } \\
\text { number }\end{array}$ & $\begin{array}{c}\text { Atom } \\
\text { number }\end{array}$ & Type & X & Y & Z \\
\hline 1. & 8. & 0. & -0.255819 & 0.169524 & 0.639321 \\
\hline 2. & 6. & 0. & -2.508520 & 0.502030 & 1.464756 \\
\hline 3. & 6. & 0. & -3.850058 & 0.119057 & 1.485981 \\
\hline 4. & 6. & 0. & -4.297094 & -0.934772 & 0.685238 \\
\hline 5. & 6. & 0. & -3.379352 & -1.617723 & -0.124045 \\
\hline 6. & 6. & 0. & -2.036055 & -1.238957 & -0.149817 \\
\hline 7. & 6. & 0. & -1.601899 & -0.160126 & 0.642739 \\
\hline 8. & 8. & 0. & -1.073333 & -1.860513 & -0.905844 \\
\hline 9. & 6. & 0. & -1.476264 & -2.893079 & -1.804794 \\
\hline 10. & 6. & 0. & -5.768772 & -1.333359 & 0.687076 \\
\hline
\end{tabular}




\begin{tabular}{|c|c|c|c|c|c|}
\hline 11. & 6. & 0. & -6.635278 & -0.474029 & -0.252049 \\
\hline 12. & 6. & 0. & -6.291868 & -0.598702 & -1.738379 \\
\hline 13. & 8. & 0. & -5.961457 & -2.692310 & 0.295893 \\
\hline 14. & 6. & 0. & 1.709356 & 3.725579 & -0.084143 \\
\hline 15. & 6. & 0. & 2.182141 & 2.531808 & -0.914007 \\
\hline 16. & 6. & 0. & 1.614518 & 1.215517 & -0.374593 \\
\hline 17. & 6. & 0. & 0.109572 & 1.309471 & -0.105618 \\
\hline 18. & 8. & 0. & -0.192290 & 2.458159 & 0.667079 \\
\hline 19. & 6. & 0. & 0.186592 & 3.696818 & 0.034634 \\
\hline 20. & 6. & 0. & -0.396254 & 4.806222 & 0.898210 \\
\hline 21. & 8. & 0. & -1.810090 & 4.731963 & 0.950219 \\
\hline 22. & 8. & 0. & 2.100998 & 4.945402 & -0.689556 \\
\hline 23. & 8. & 0. & 3.597803 & 2.554809 & -0.896156 \\
\hline 24. & 8. & 0. & 1.813914 & 0.193947 & -1.358607 \\
\hline 25. & 6. & 0. & 3.829400 & -2.315033 & 0.403820 \\
\hline 26. & 6. & 0. & 2.445809 & -1.703147 & 0.157819 \\
\hline 27. & 6. & 0. & 2.712785 & -0.835858 & -1.084598 \\
\hline 28. & 8. & 0. & 4.026353 & -0.308229 & -0.885846 \\
\hline 29. & 6. & 0. & 4.768133 & -1.161117 & 0.015046 \\
\hline 30. & 8. & 0. & 4.037224 & -3.382337 & -0.532996 \\
\hline 31. & 8. & 0. & 1.499167 & -2.724570 & -0.058330 \\
\hline 32. & 8. & 0. & 5.346579 & -3.451886 & 1.904202 \\
\hline 33. & 6. & 0. & 4.061915 & -2.863862 & 1.812056 \\
\hline 34. & 1. & 0. & -2.137587 & 1.301946 & 2.094956 \\
\hline 35. & 1. & 0. & -4.546878 & 0.639828 & 2.137120 \\
\hline 36. & 1. & 0. & -3.739053 & -2.444950 & -0.722762 \\
\hline 37. & 1. & 0. & -0.564141 & -3.212290 & -2.309225 \\
\hline 38. & 1. & 0. & -2.194090 & -2.515016 & -2.540345 \\
\hline 39. & 1. & 0. & -1.913460 & -3.743796 & -1.269859 \\
\hline 40. & 1. & 0. & -6.151497 & -1.181208 & 1.709199 \\
\hline 41. & 1. & 0. & -7.677242 & -0.773597 & -0.089262 \\
\hline 42. & 1. & 0. & -6.549481 & 0.570154 & 0.070907 \\
\hline 43. & 1. & 0. & -5.270949 & -0.263665 & -1.945816 \\
\hline 44. & 1. & 0. & -6.385500 & -1.635347 & -2.072429 \\
\hline 45. & 1. & 0. & -6.971835 & 0.013479 & -2.338709 \\
\hline 46. & 1. & 0. & -5.488712 & -3.249702 & 0.928520 \\
\hline 47. & 1. & 0. & 2.138359 & 3.631047 & 0.927112 \\
\hline 48. & 1. & 0. & 1.802943 & 2.663215 & -1.941582 \\
\hline 49. & 1. & 0. & 2.115413 & 0.979440 & 0.569707 \\
\hline 50. & 1. & 0. & -0.444752 & 1.336304 & -1.057102 \\
\hline 51. & 1. & 0. & -0.266893 & 3.754744 & -0.967164 \\
\hline 52. & 1. & 0. & 0.052845 & 4.747296 & 1.902962 \\
\hline 53. & 1. & 0. & -0.131246 & 5.772670 & 0.463851 \\
\hline
\end{tabular}




\begin{tabular}{|l|l|l|l|l|l|}
\hline 54. & 1. & 0. & -2.019268 & 3.819110 & 1.193441 \\
\hline 55. & 1. & 0. & 3.042626 & 4.850629 & -0.895837 \\
\hline 56. & 1. & 0. & 3.926261 & 1.668804 & -1.132115 \\
\hline 57. & 1. & 0. & 2.161579 & -1.069238 & 1.007954 \\
\hline 58. & 1. & 0. & 2.684123 & -1.468932 & -1.979492 \\
\hline 59. & 1. & 0. & 5.659650 & -1.540854 & -0.489585 \\
\hline 60. & 1. & 0. & 5.072524 & -0.570561 & 0.885615 \\
\hline 61. & 1. & 0. & 3.173934 & -3.821159 & -0.618811 \\
\hline 62. & 1. & 0. & 0.630183 & -2.309578 & -0.218920 \\
\hline 63. & 1. & 0. & 5.410979 & -4.033834 & 1.130806 \\
\hline 64. & 1. & 0. & 4.015583 & -2.062648 & 2.557463 \\
\hline 65. & 1. & 0. & 3.260342 & -3.584388 & 2.042389 \\
\hline
\end{tabular}

The fragmentation patterns acquired from the $\mathrm{MS}^{2}$ spectra were summarized. In the negative mode, the neutral loss of $162 \mathrm{u}, 146 \mathrm{u}$ and $132 \mathrm{u}$ were observed in $\mathrm{MS}^{2}$ spectra of these isolated phenolic glycosides, indicating the presence of glucose, rhamnose and apiose, respectively (figure 7). Proposed fragmentations of compound 1-27 were as follows:

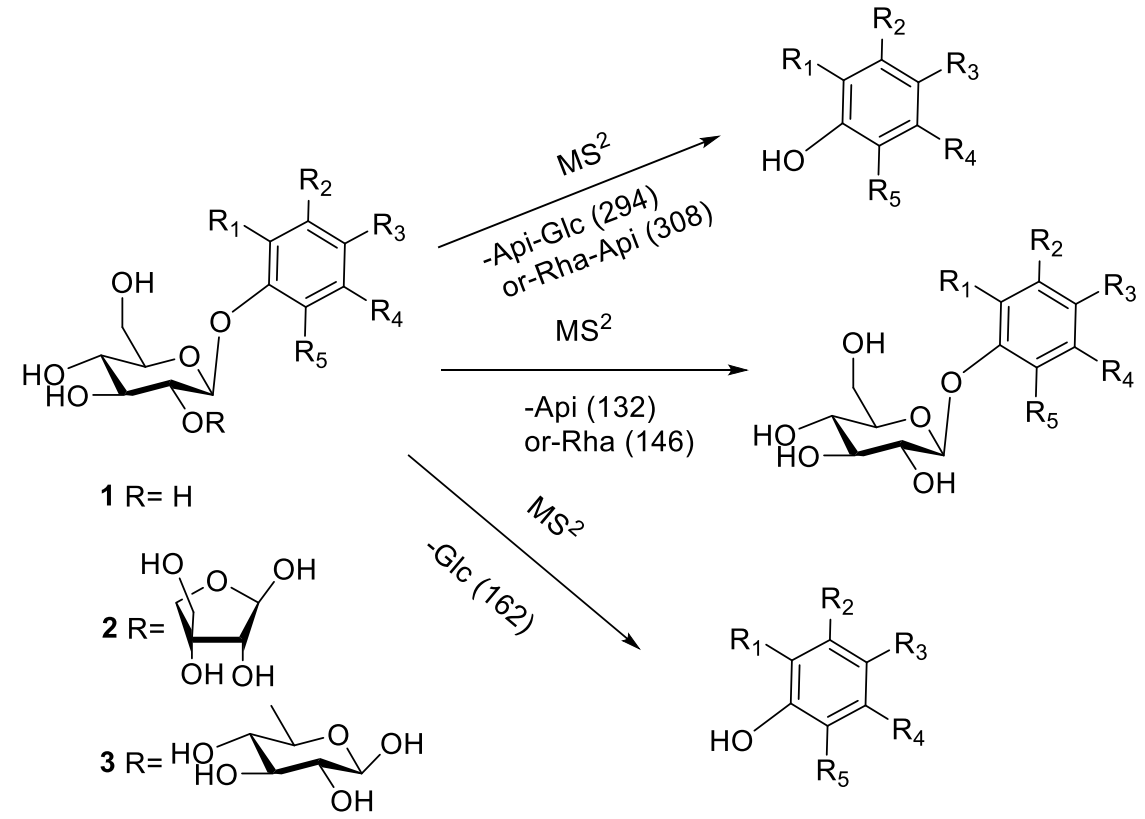

The fragmentation patterns of phenolic glycosides 
FH36 \#121 RT: 0.35 AV: 1 NL: 1.25E8

T: FTMS - p ESI Full ms [100.0000-1000.0000]

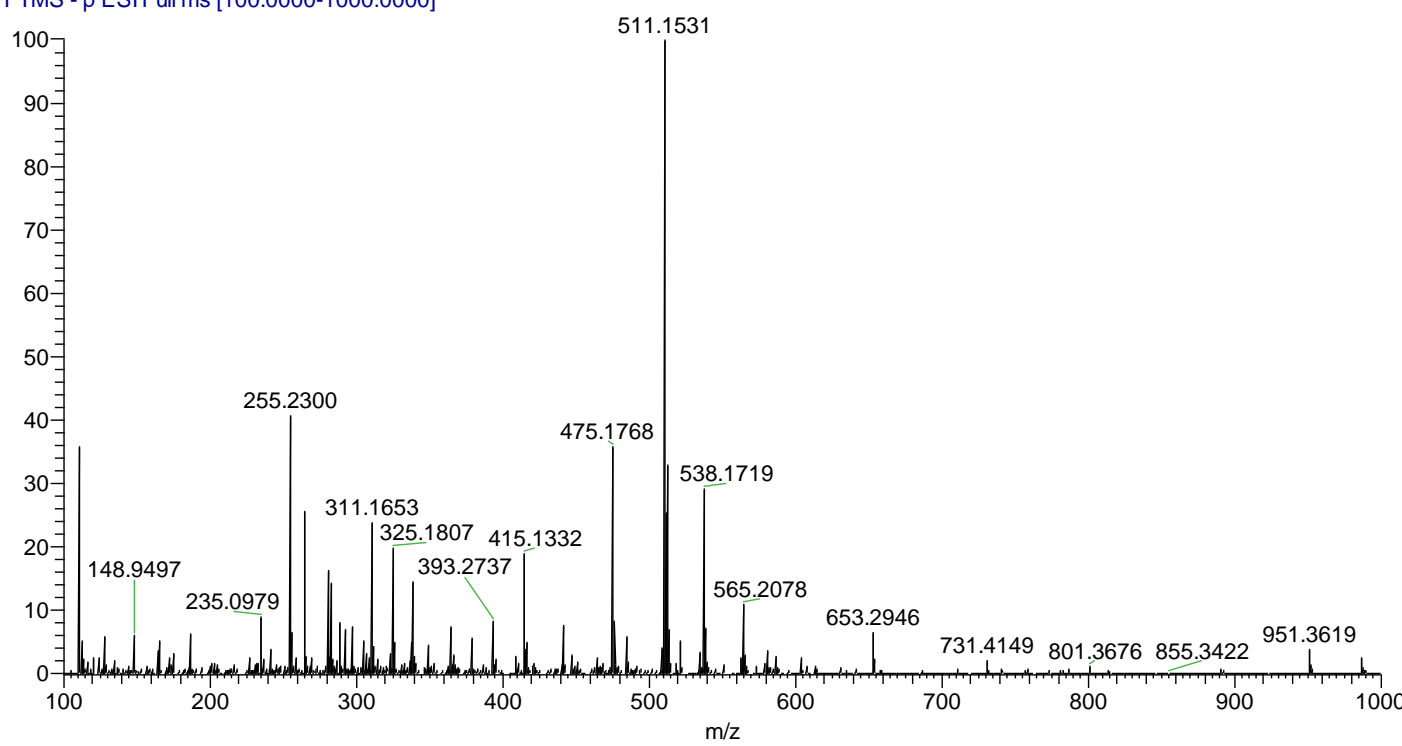

HRESIMS in the negative mode of compound 1

FH36 \#134 RT: 0.39 AV: 1 NL: 1.12E7

T: FTMS - p ESI Full ms2 475.1810@hcd25.00 [!

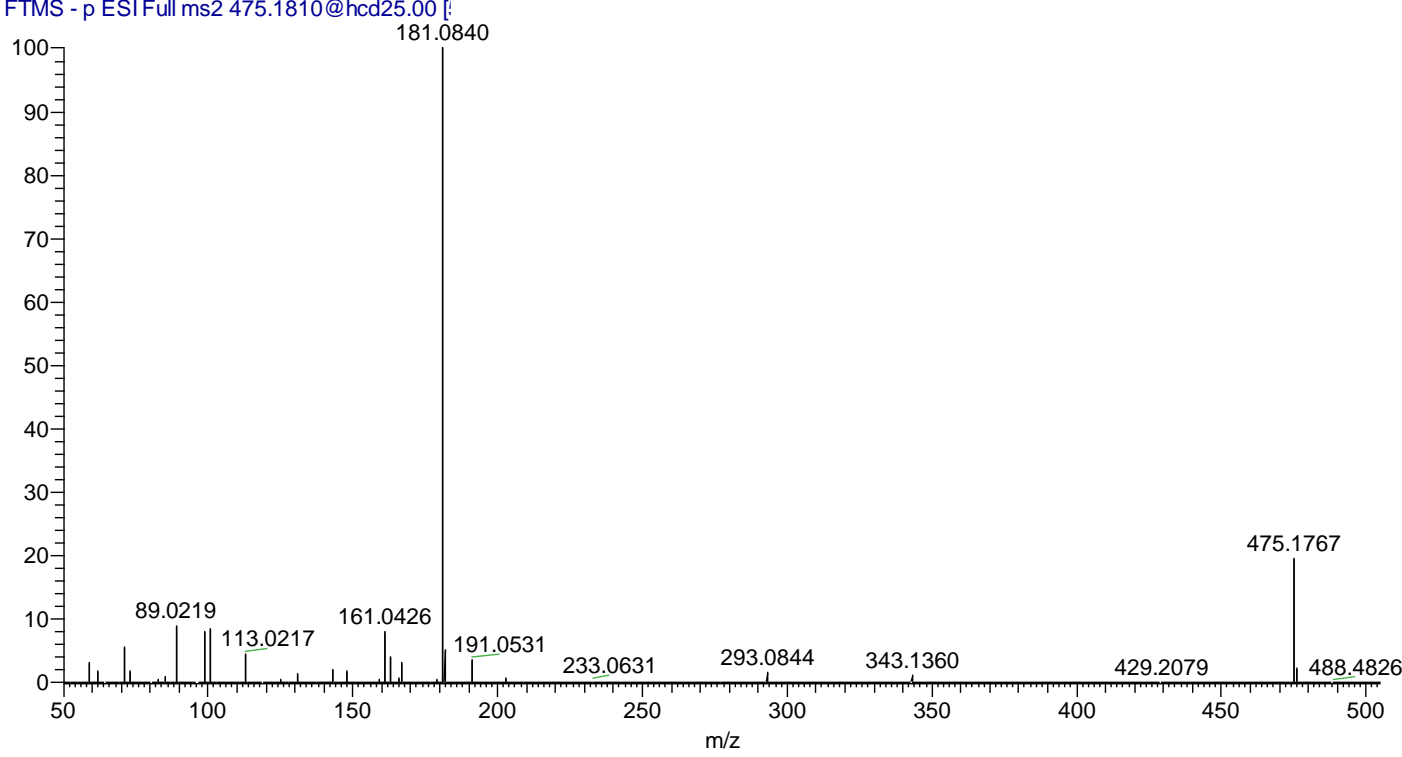

MS/MS in the negative mode of compound $\mathbf{1}$

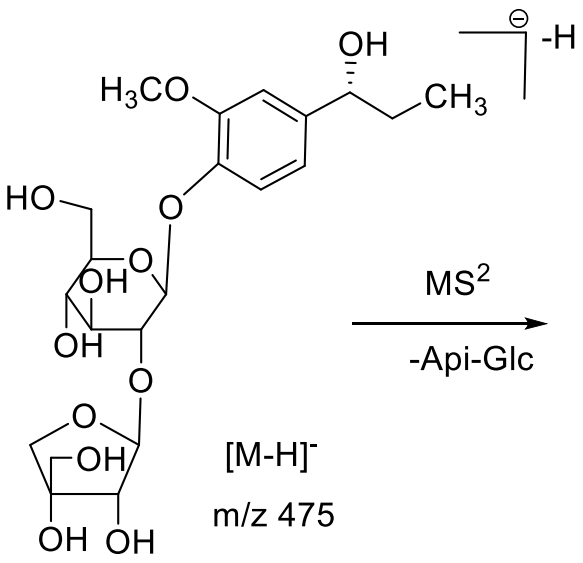<smiles>CC[C@H](O)c1ccc(O)c(OC)c1</smiles>

$\mathrm{m} / \mathrm{z} 181$

Proposed fragmentation of compound $\mathbf{1}$ 
FH32 \#123 RT: 0.36 AV: 1 NL: 1.25E8

T: FTMS - p ESI Full ms [100.0000-1000.0000]

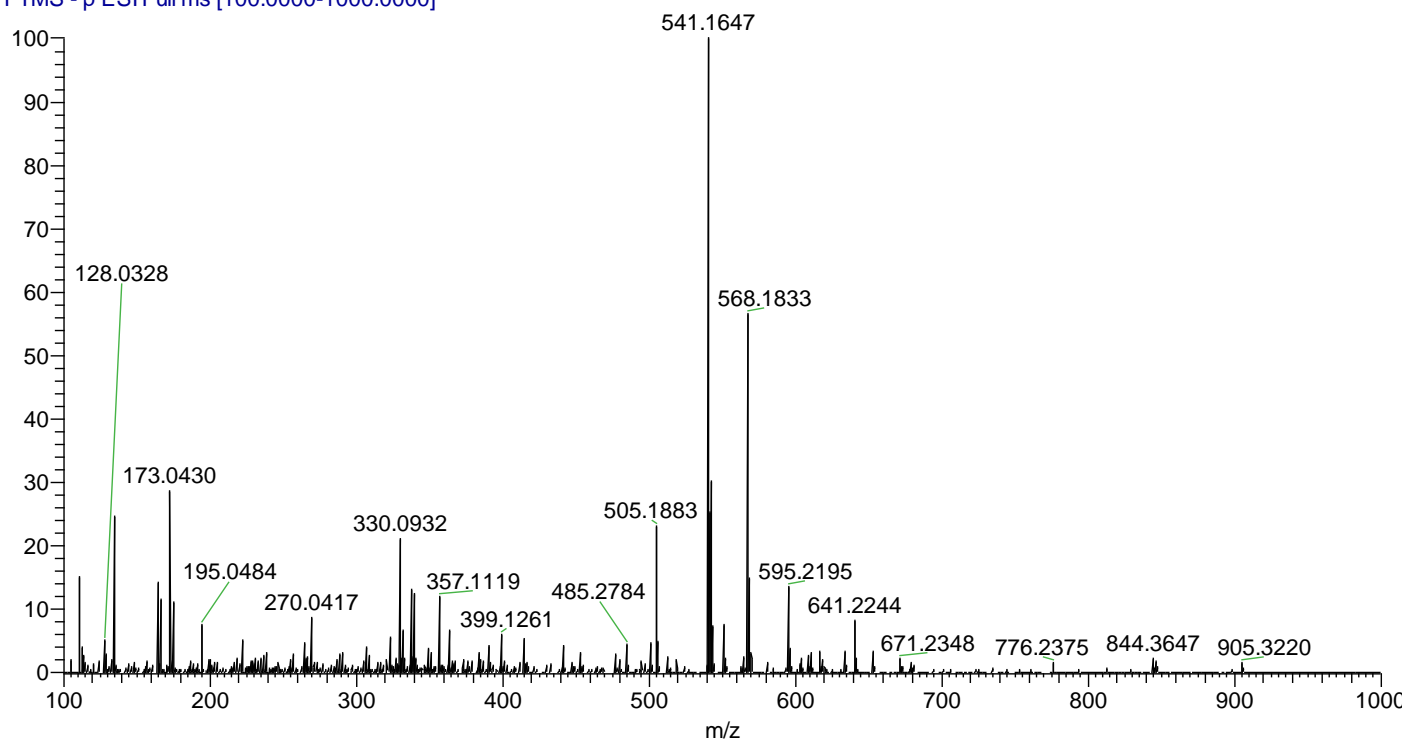

HRESIMS in the negative mode of compound 2

FH32 \#122 RT: 0.35 AV: 1 NL: 7.46E6

T: FTMS - p ESI Full ms2 505.1916@hcd25.00 [:

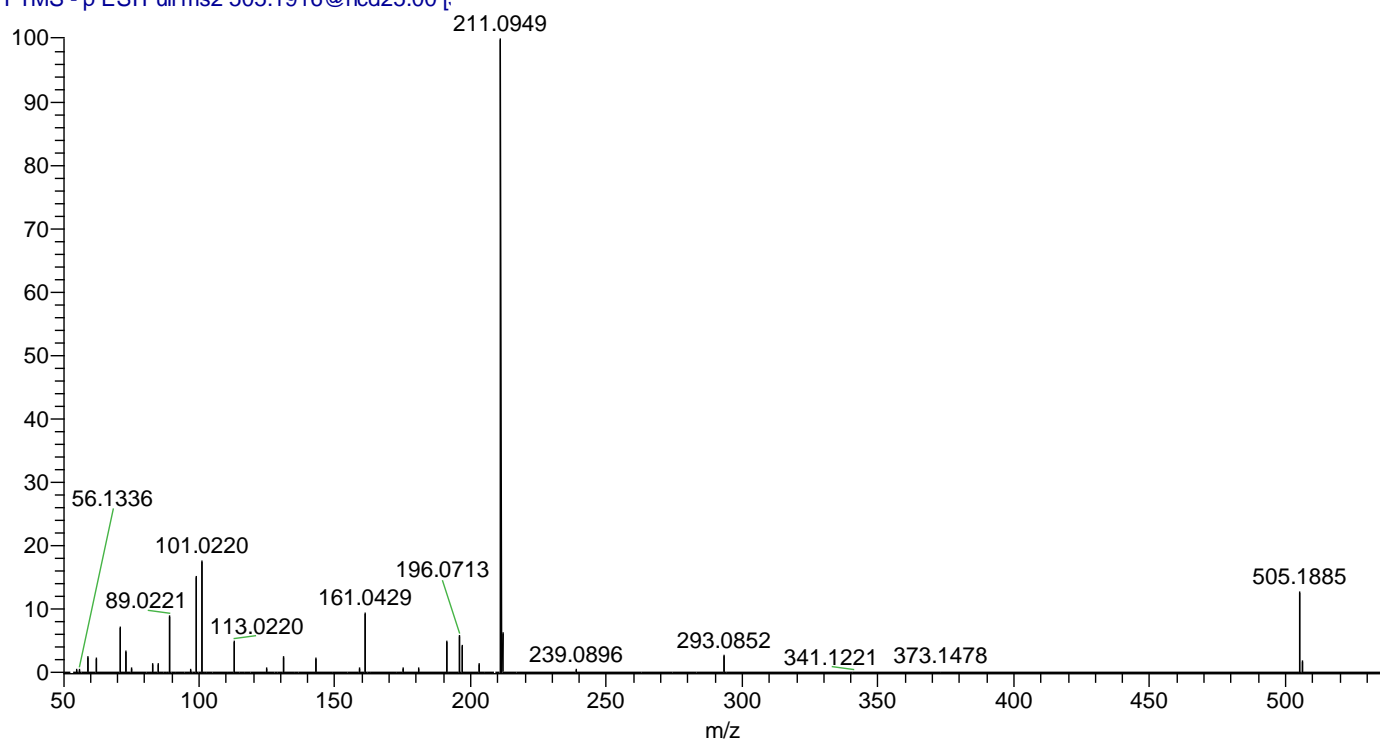

MS/MS in the negative mode of compound 2

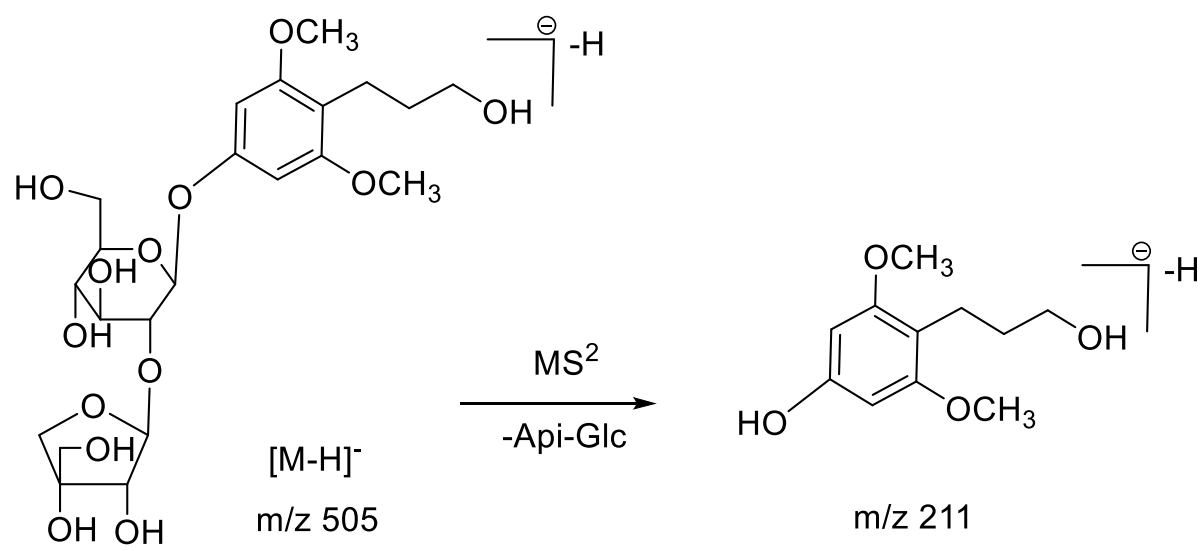

Proposed fragmentation of compound $\mathbf{2}$ 
FH59 \#119 RT: 0.34 AV: 1 NL: $1.86 E 8$

T: FTMS - p ESI Full ms [100.0000-1000.0000]

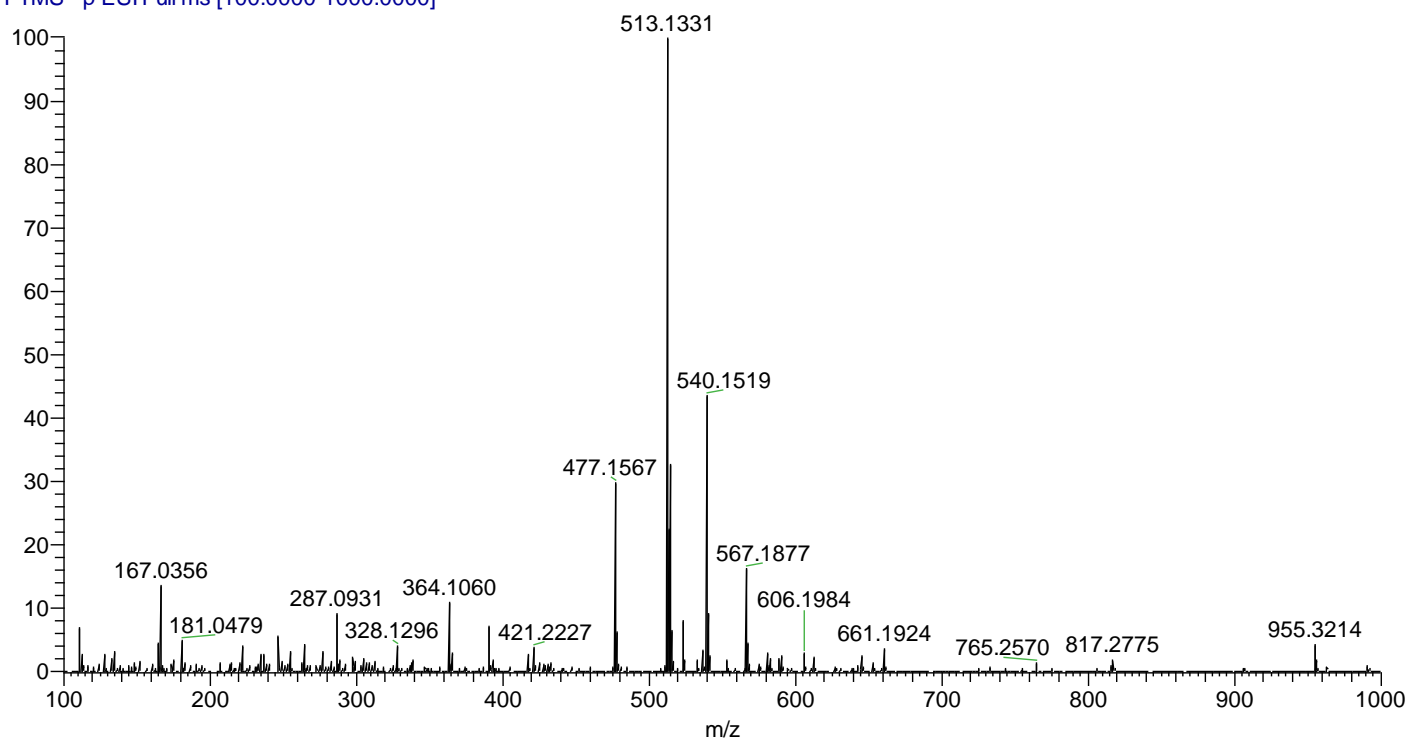

HRESIMS in the negative mode of compound 3

FH59 \#120 RT: 0.35 AV: 1 NL: $2.42 E 7$

T: FTMS - p ESI Full ms2 477.1603@hcd25.00 [?

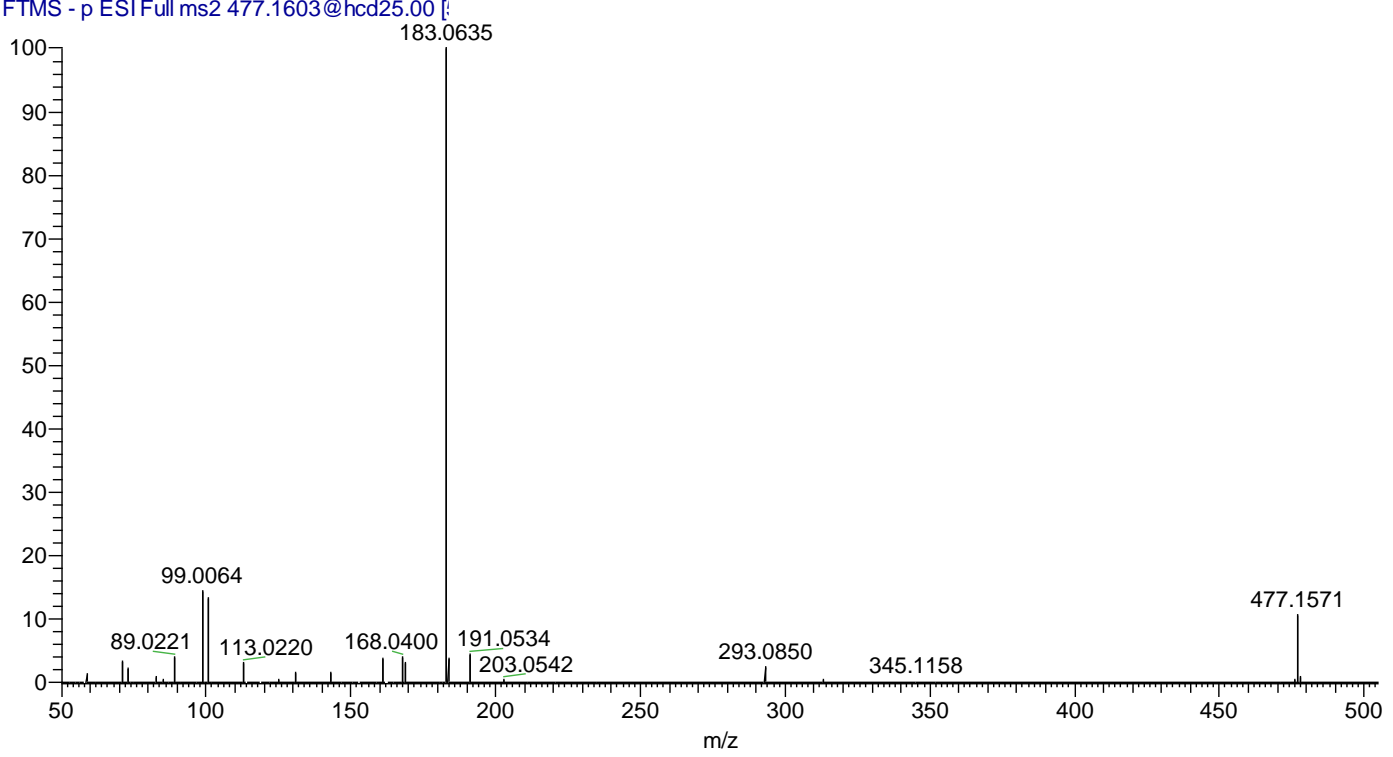

MS/MS in the negative mode of compound $\mathbf{3}$<smiles>COc1cc(CO)cc(OC)c1O</smiles>

Proposed fragmentation of compound $\mathbf{3}$ 


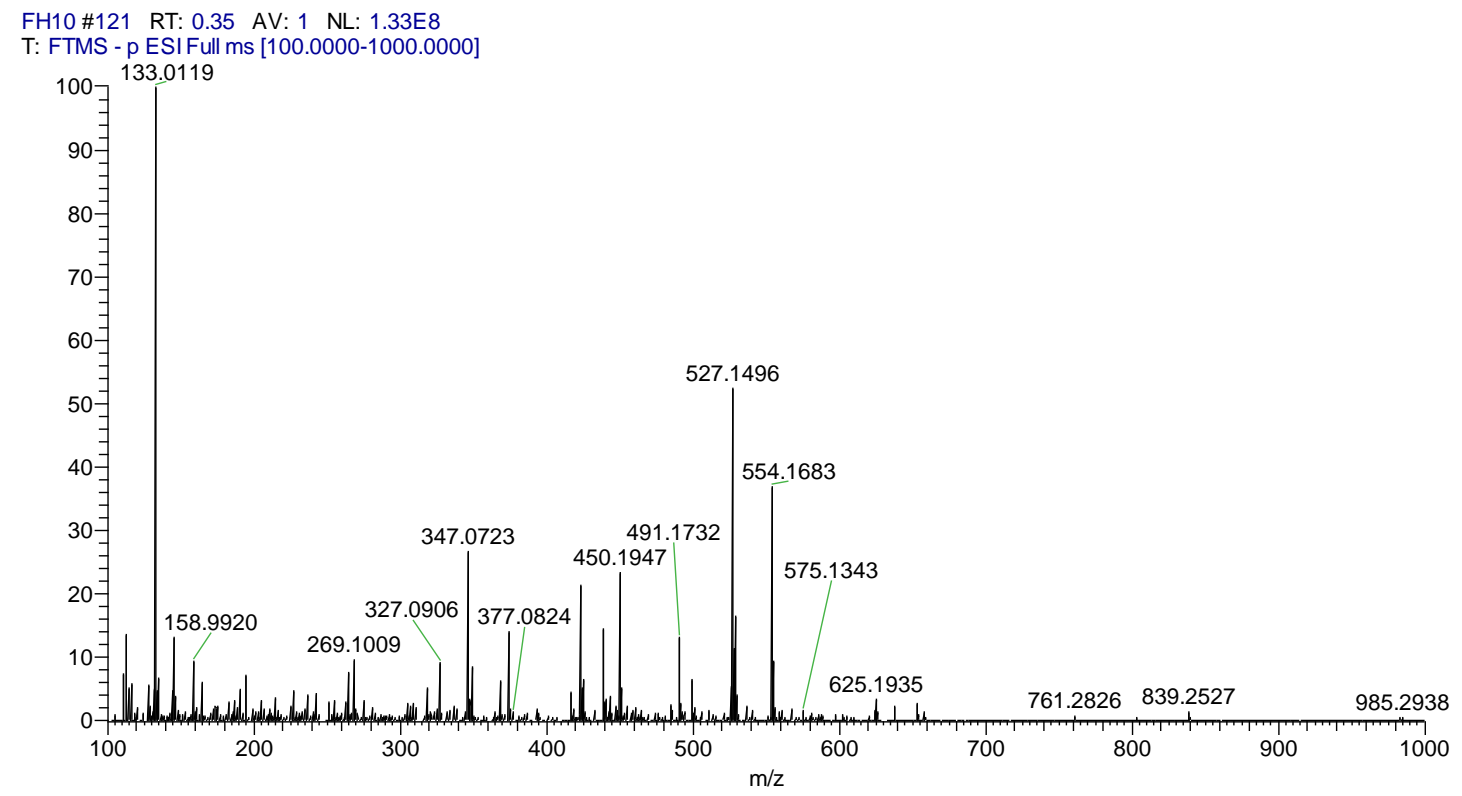

HRESIMS in the negative mode of compound 4

FH10 \#130 RT: 0.38 AV: 1 NL: 2.10E6

T: FTMS - p ESI Full ms2 491.1759@hcd25.00 [:

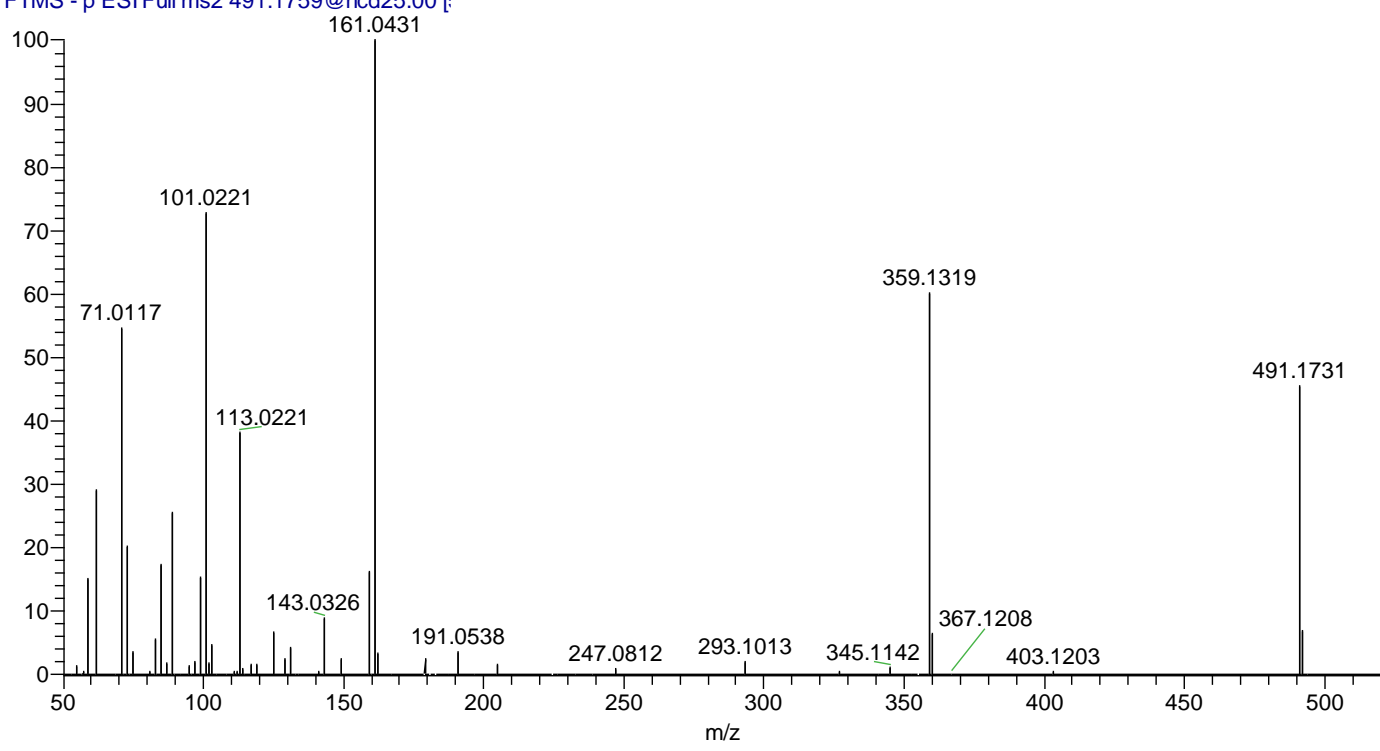

MS/MS in the negative mode of compound 4

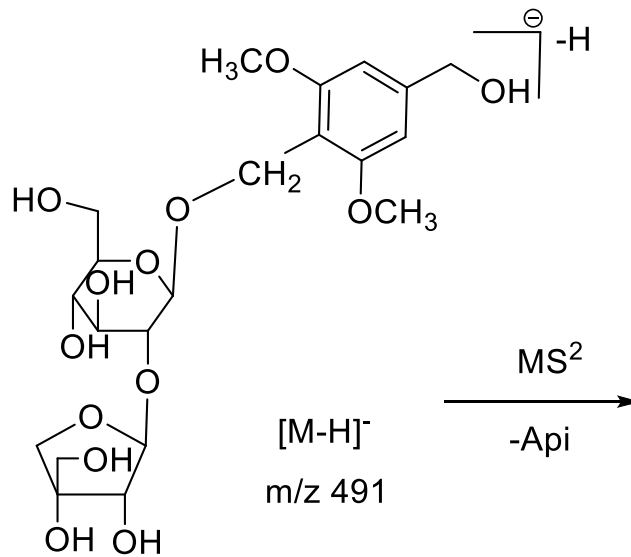<smiles>COc1cc(CO)cc(OC)c1COC1OC2(CO)OC(O)C(O2)C1O</smiles>

$\mathrm{m} / \mathrm{z} 359$

Proposed fragmentation of compound 4 


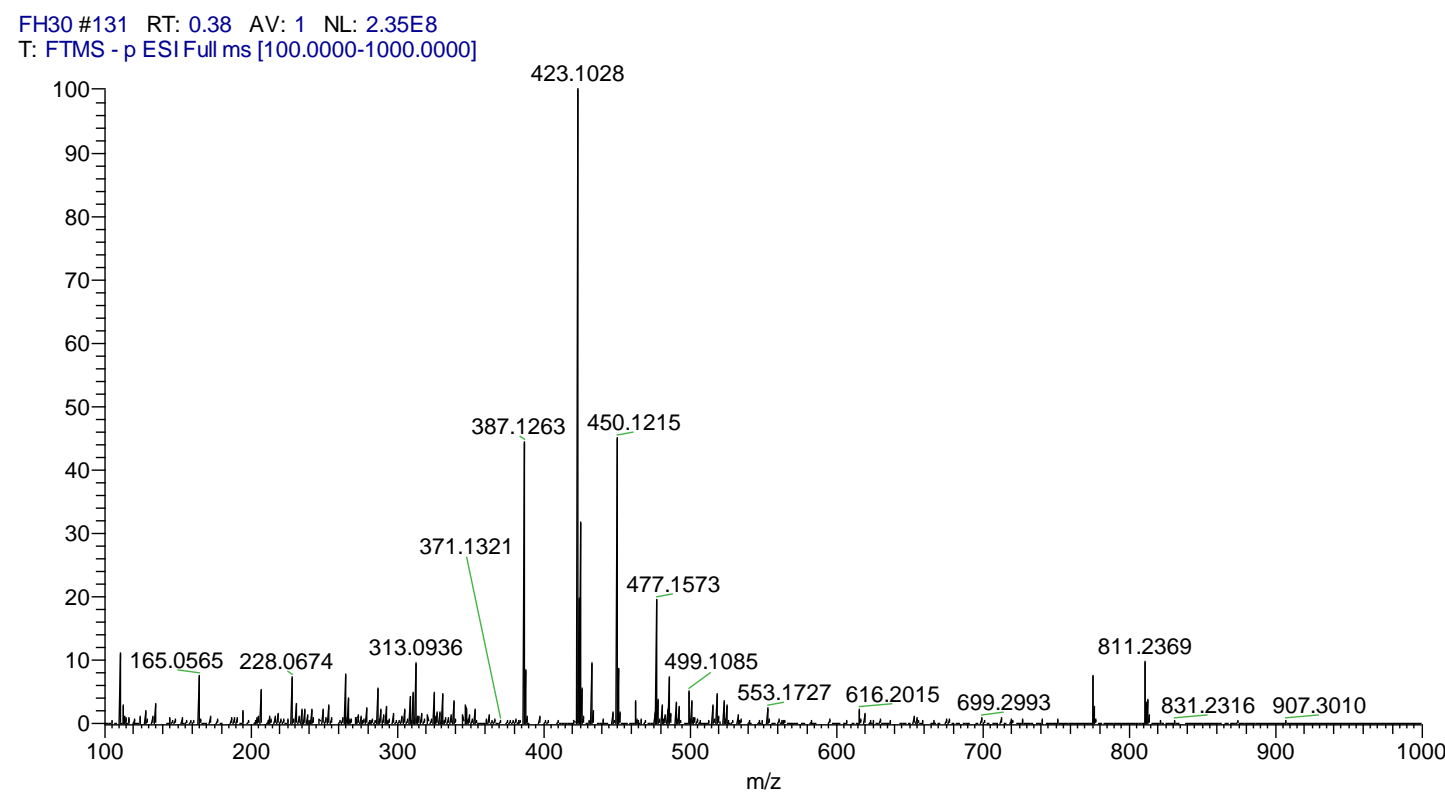

HRESIMS in the negative mode of compound $\mathbf{5}$

FH30 \#100 RT: 0.29 AV: 1 NL: 1.16E7

T: FTMS - p ESI Full ms2 387.1286@hcd25.00 [?

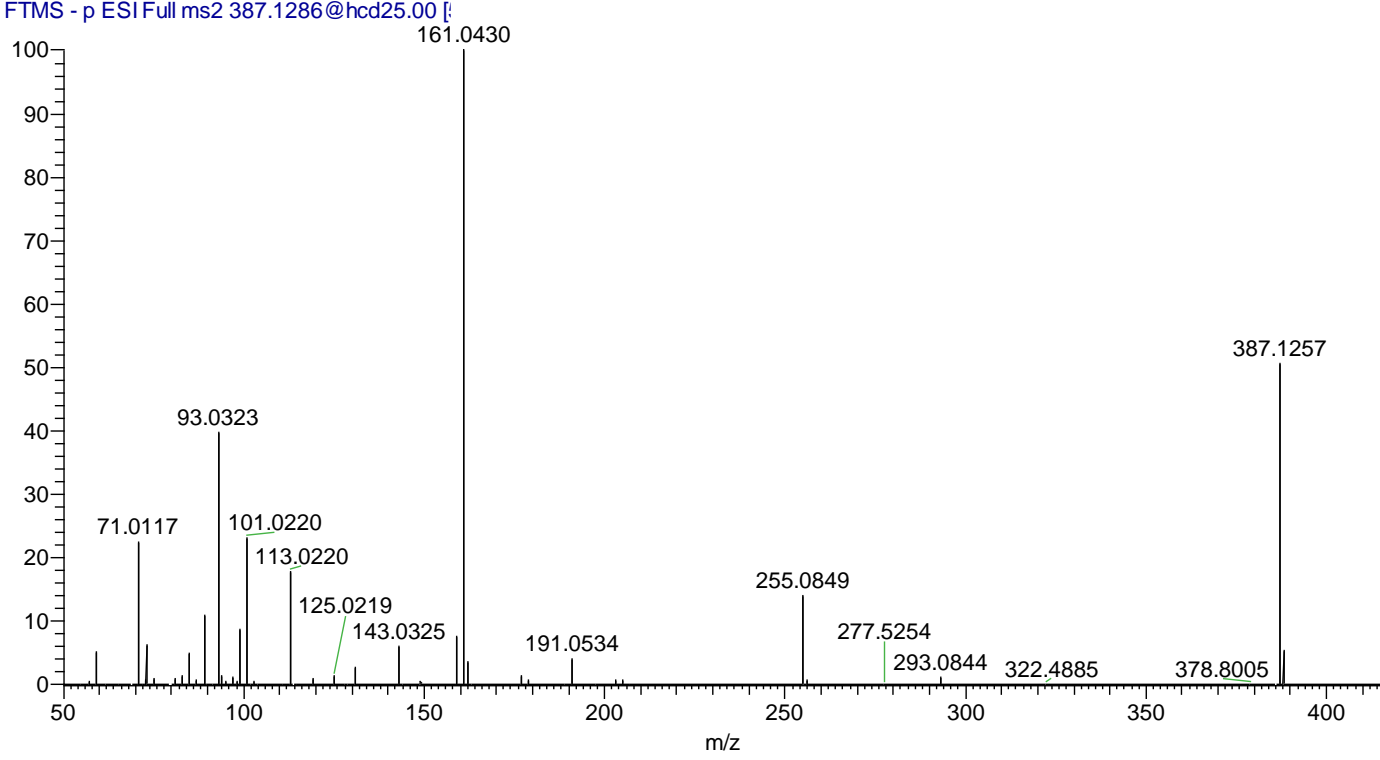

MS/MS in the negative mode of compound $\mathbf{5}$

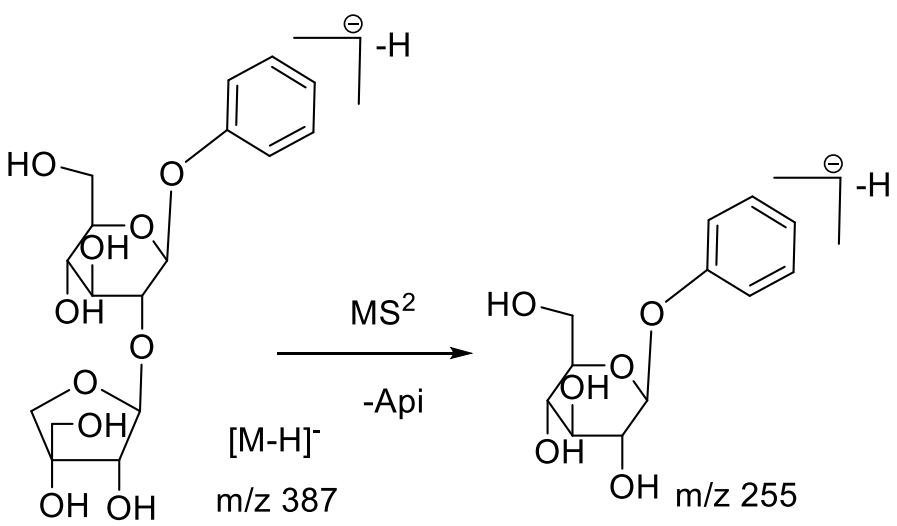

Proposed fragmentation of compound $\mathbf{5}$ 
FH49 \#113 RT: 0.33 AV: 1 NL: 2.34E8

T: FTMS - p ESI Full ms [100.0000-1000.0000]

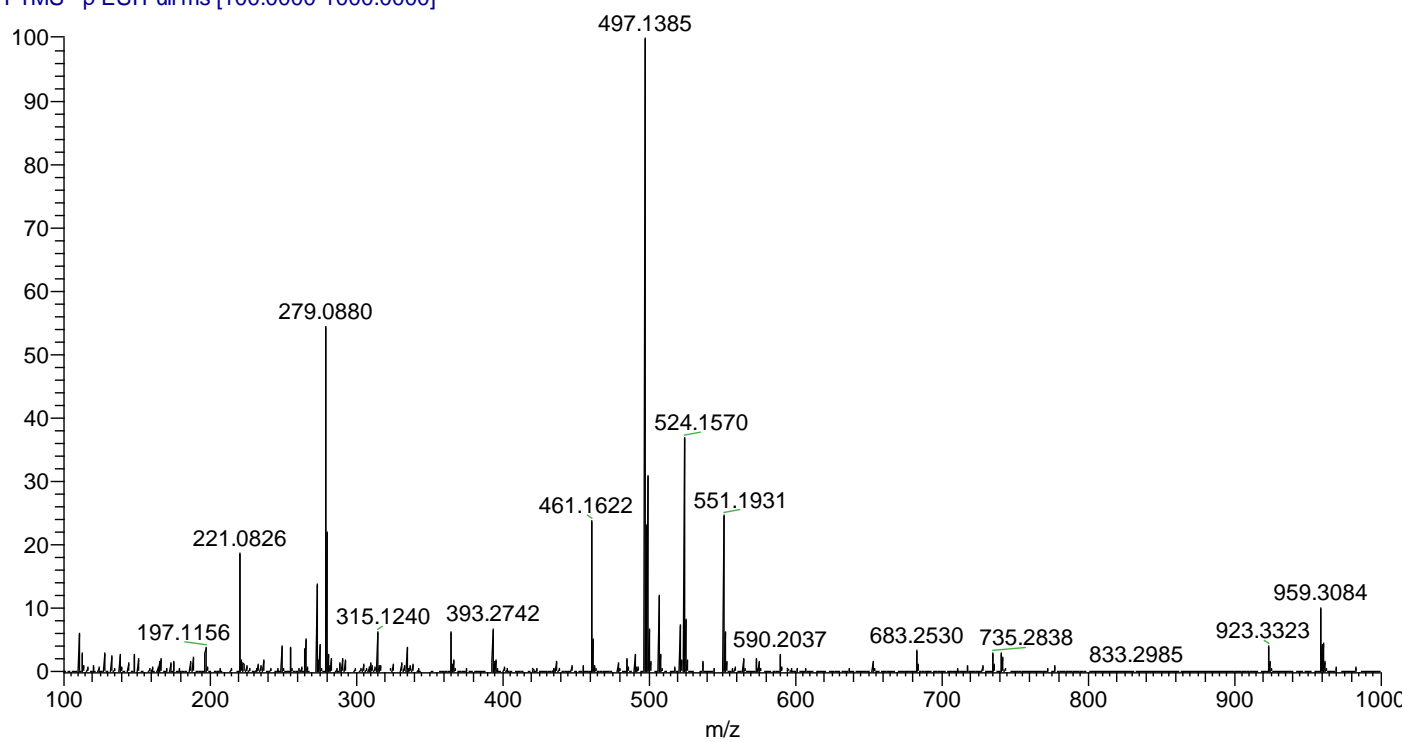

HRESIMS in the negative mode of compound 6

FH49 \#114 RT: 0.33 AV: 1 NL: 4.37E6

T: FTMS - p ESIFull ms2 461.1654@hcd25.00 [!

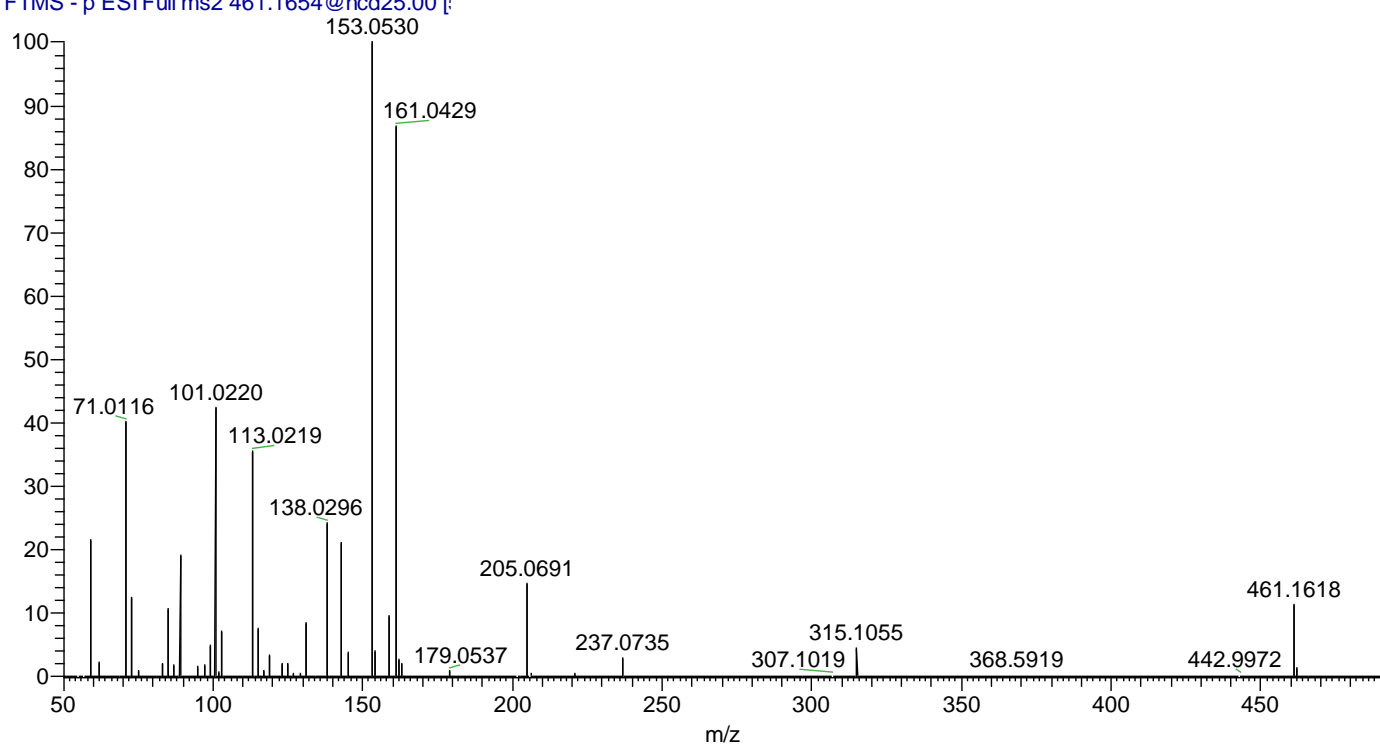

MS/MS in the negative mode of compound $\mathbf{6}$

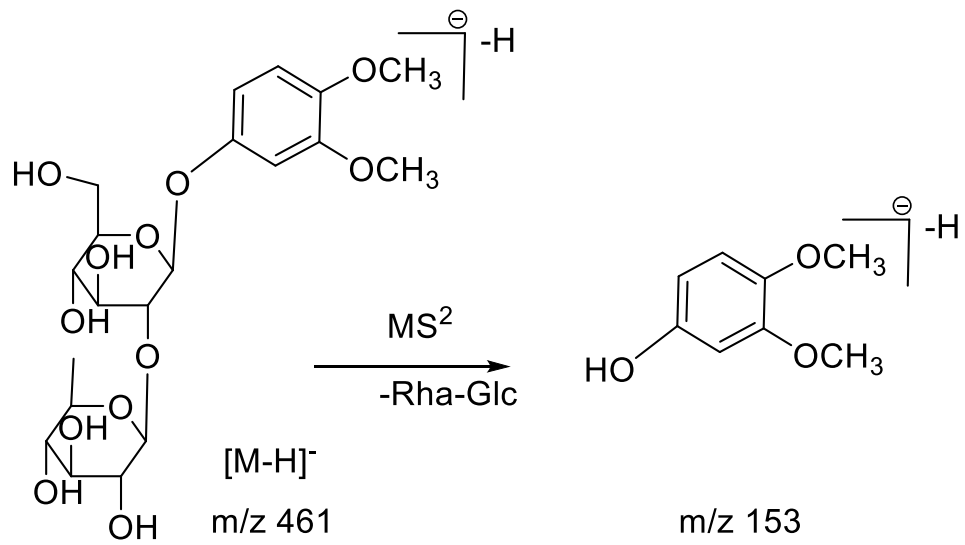

Proposed fragmentation of compound $\mathbf{6}$ 


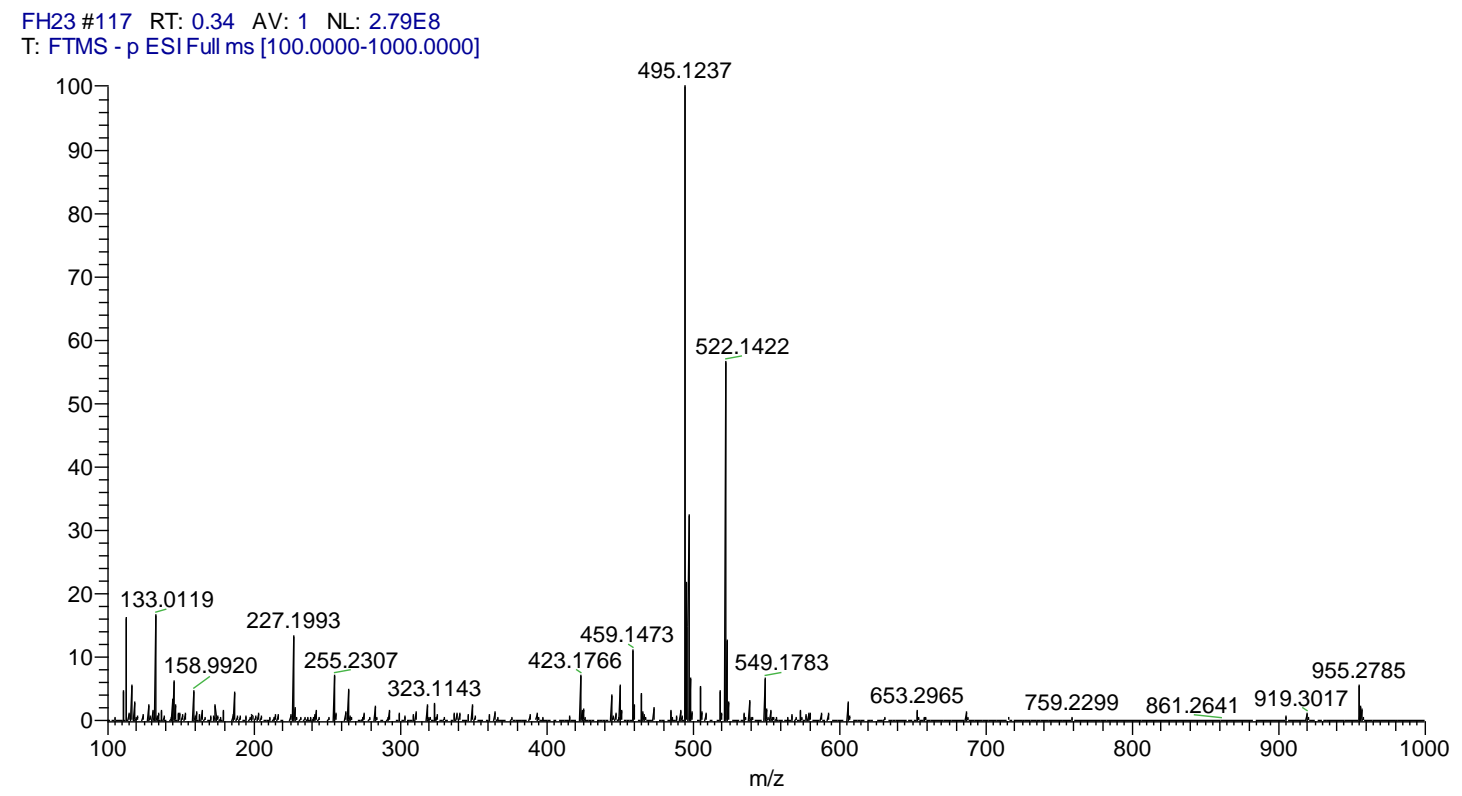

HRESIMS in the negative mode of compound 7

FH23 \#118 RT: 0.34 AV: 1 NL: $1.08 E 7$

T: FTMS - p ESI Full ms2 459.1497@hcd25.00 [:

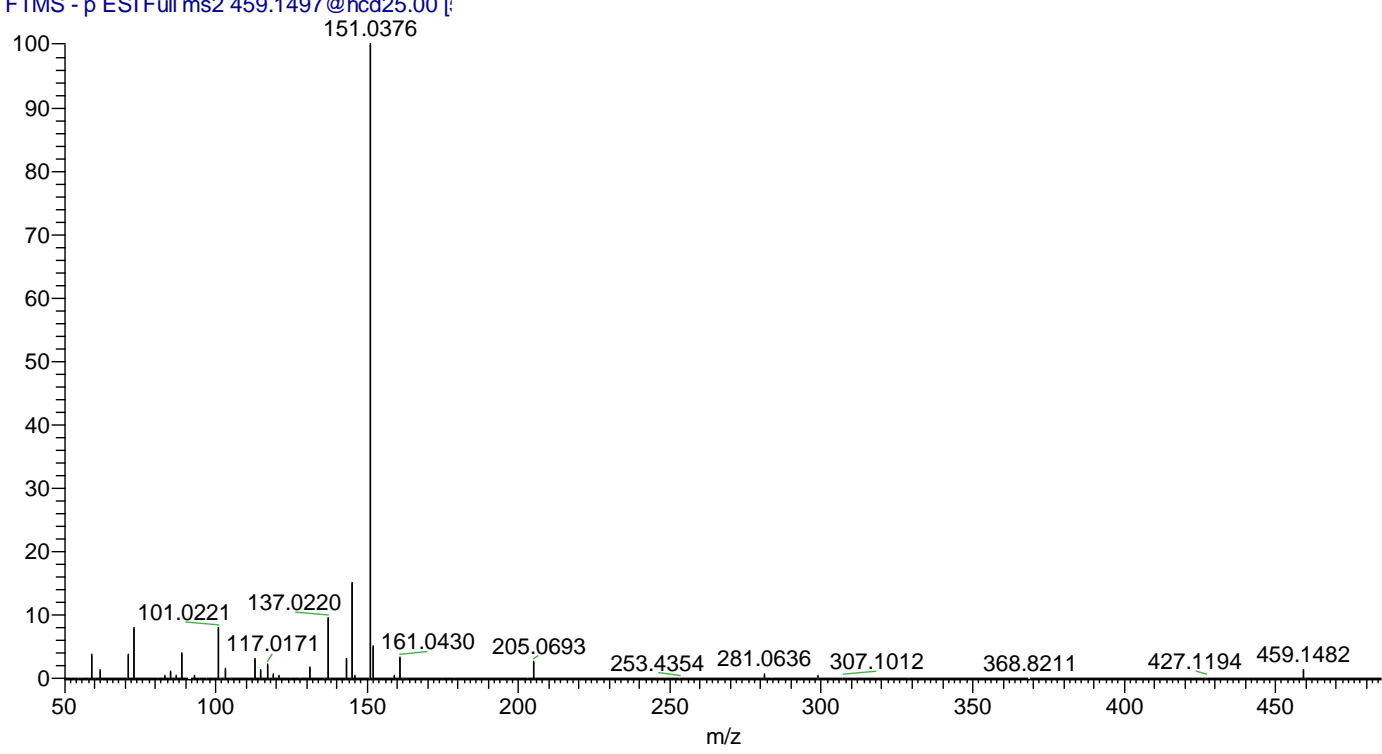

MS/MS in the negative mode of compound 7

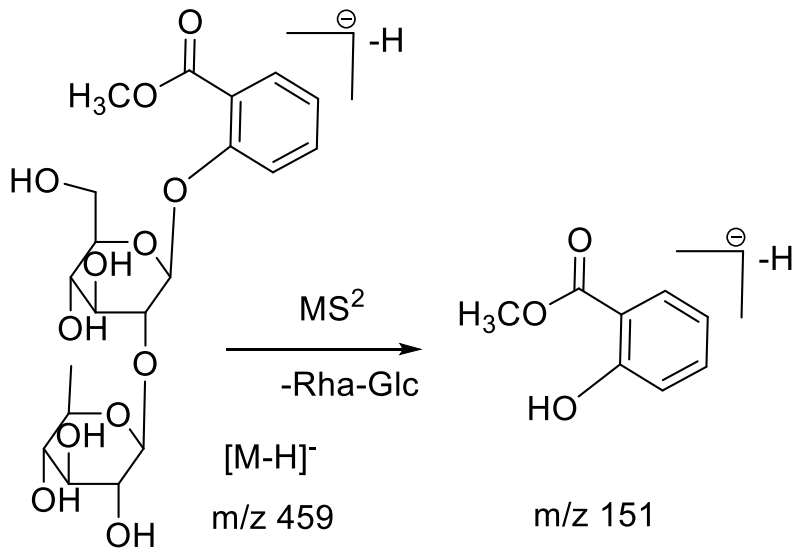

Proposed fragmentation of compound 7 
FH24 \#117 RT: 0.34 AV: 1 NL: 2.56E8

T: FTMS - p ESIFull ms [100.0000-1000.0000]

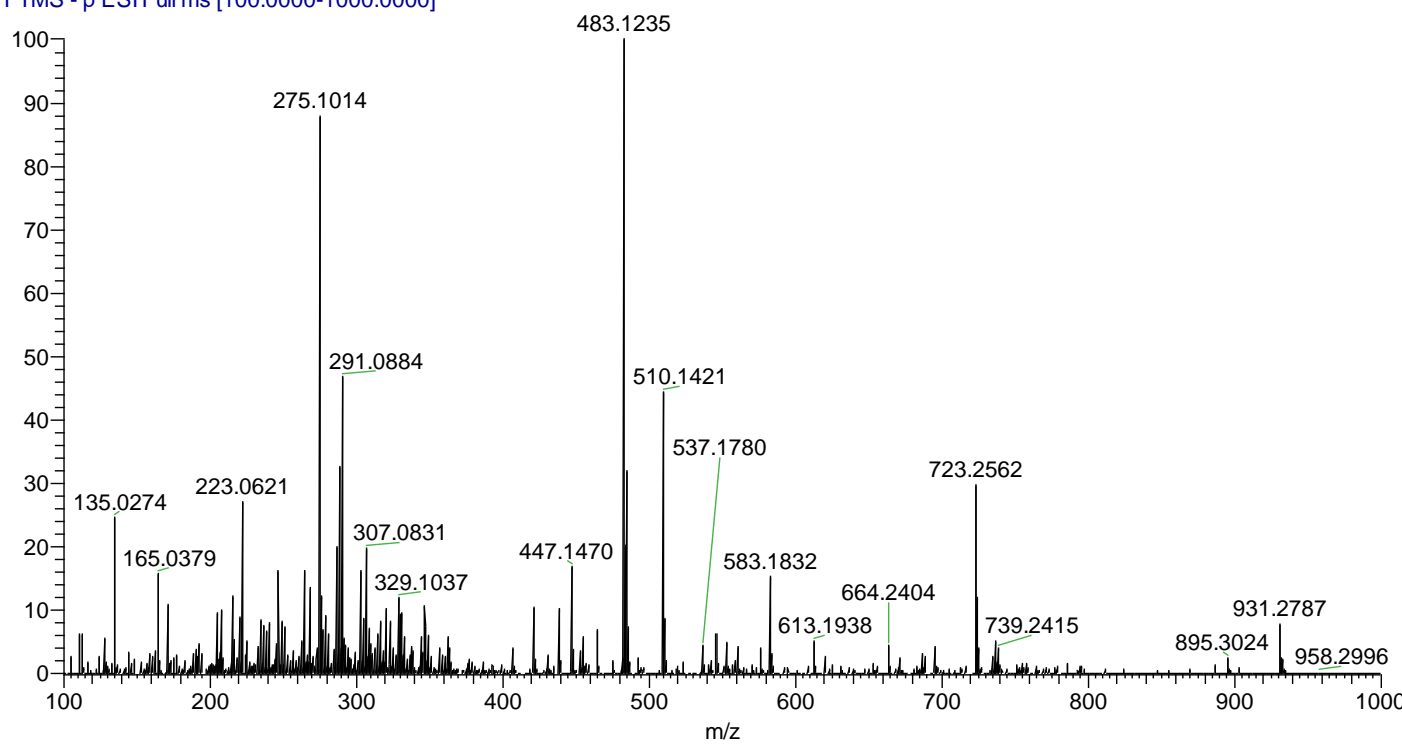

HRESIMS in the negative mode of compound 8

FH24 \#118 RT: 0.34 AV: 1 NL: 4.78E6

T: FTMS - p ESI Full ms2 447.1497@hcd25.00 [:

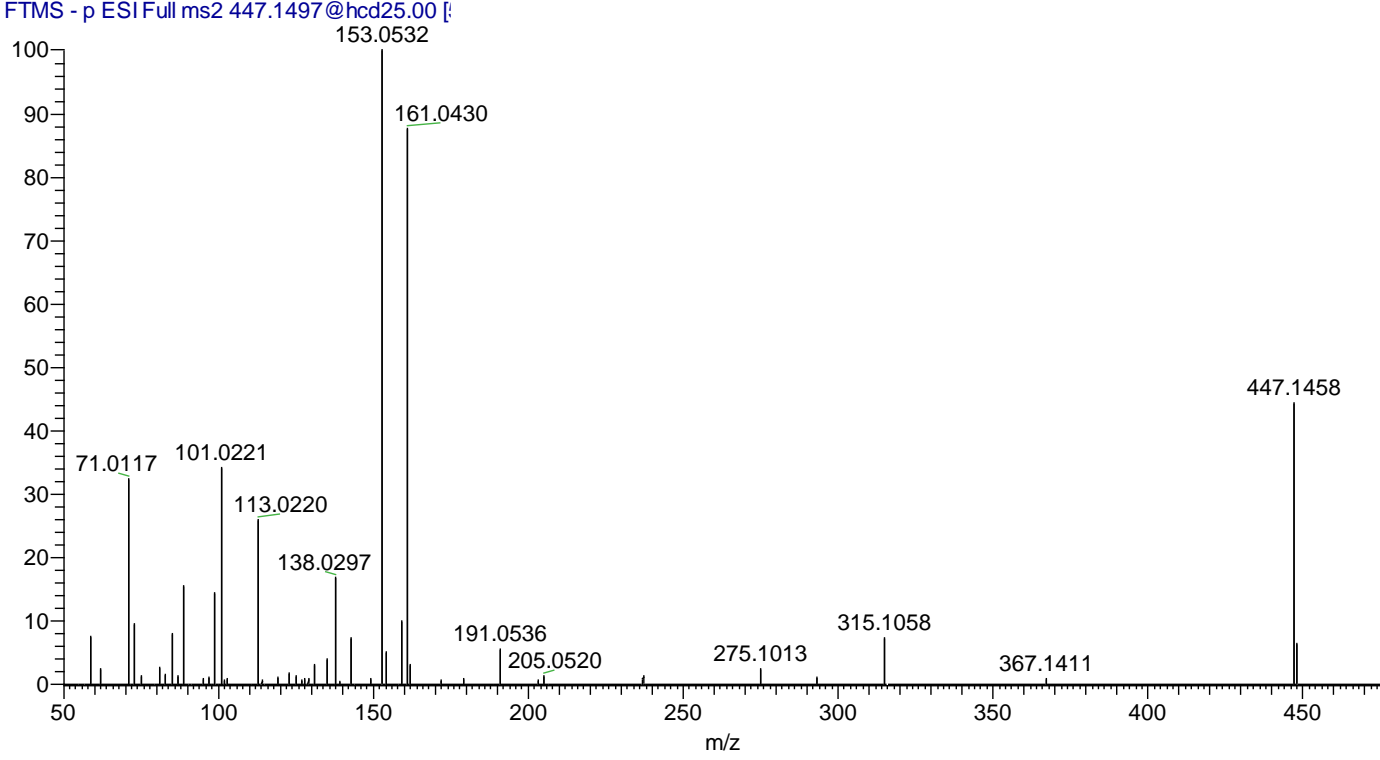

MS/MS in the negative mode of compound $\mathbf{8}$

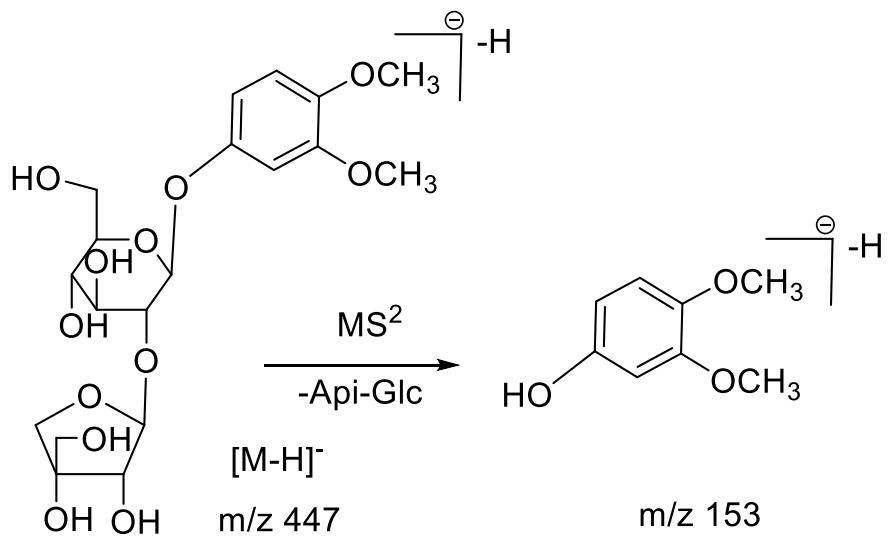

Proposed fragmentation of compound $\mathbf{8}$ 
FH26 \#121 RT: 0.35 AV: 1 NL: 1.35E8

T: FTMS - p ESI Full ms [100.0000-1000.0000]

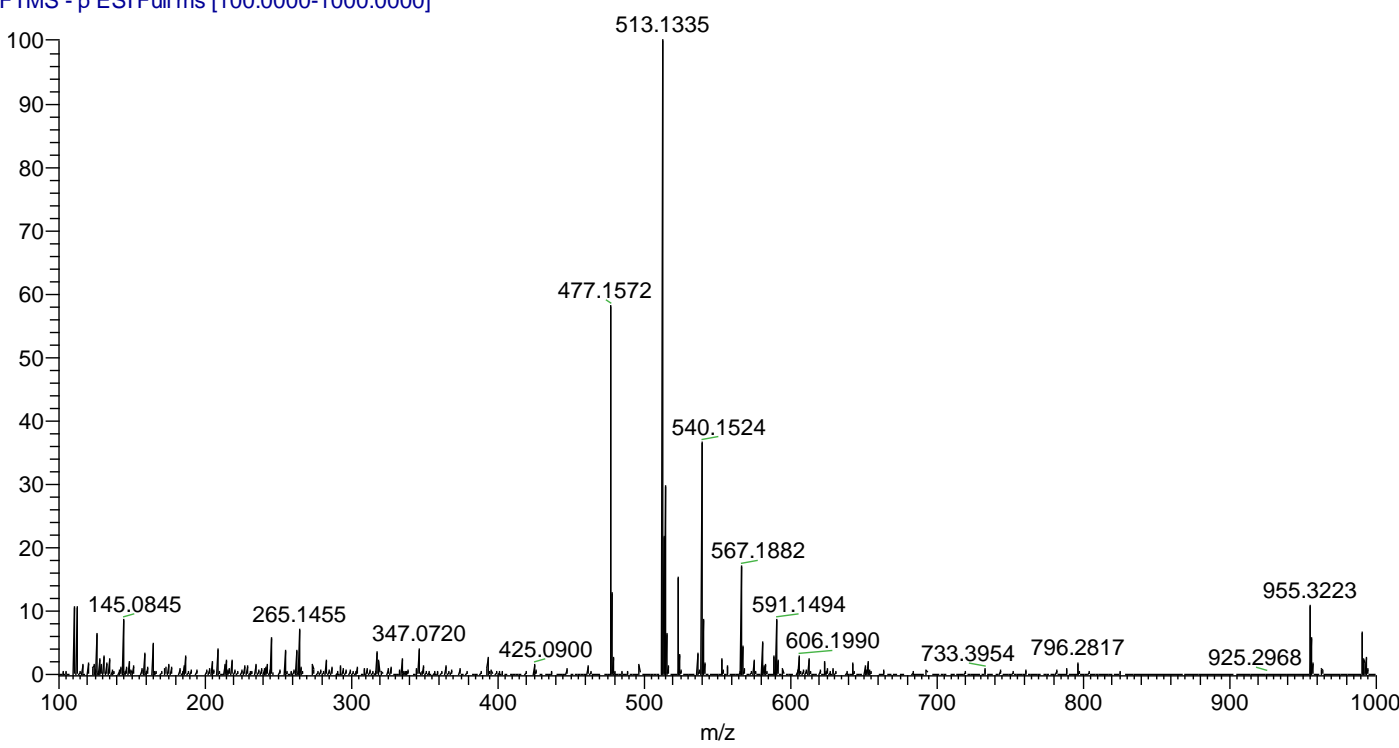

HRESIMS in the negative mode of compound 9

FH26 \#120 RT: 0.35 AV: 1 NL: $2.11 \mathrm{E} 7$

T: FTMS - p ESI Full ms2 477.1603@hcd25.00 [!

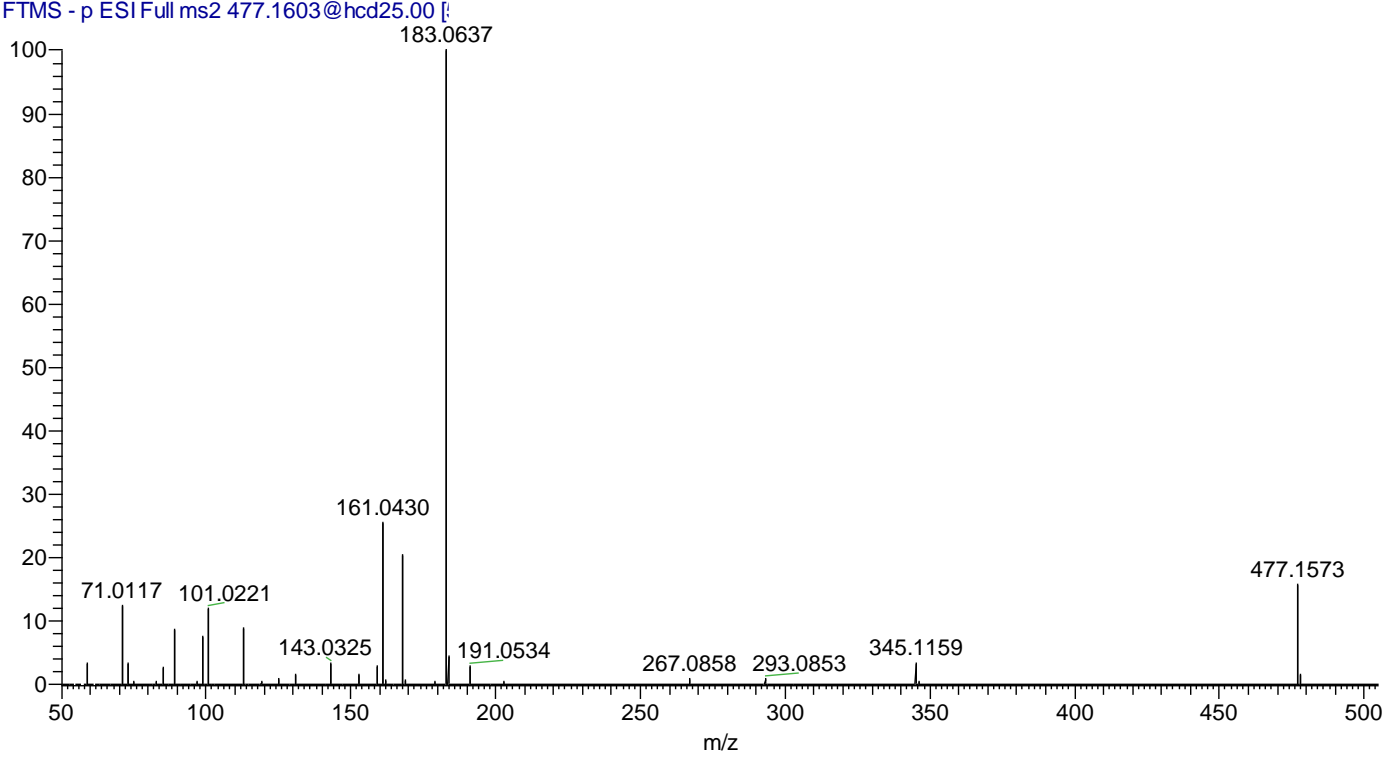

MS/MS in the negative mode of compound 9

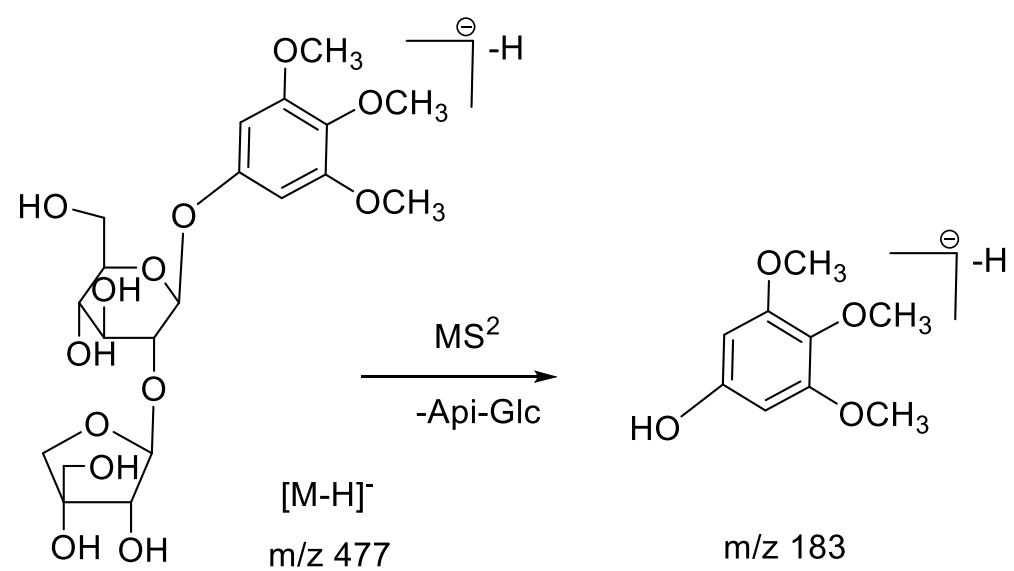

Proposed fragmentation of compound 9 
FH27 \#133 RT: 0.38 AV: 1 NL: $3.52 E 8$

T: FTMS - p ESI Full ms [100.0000-1000.0000]

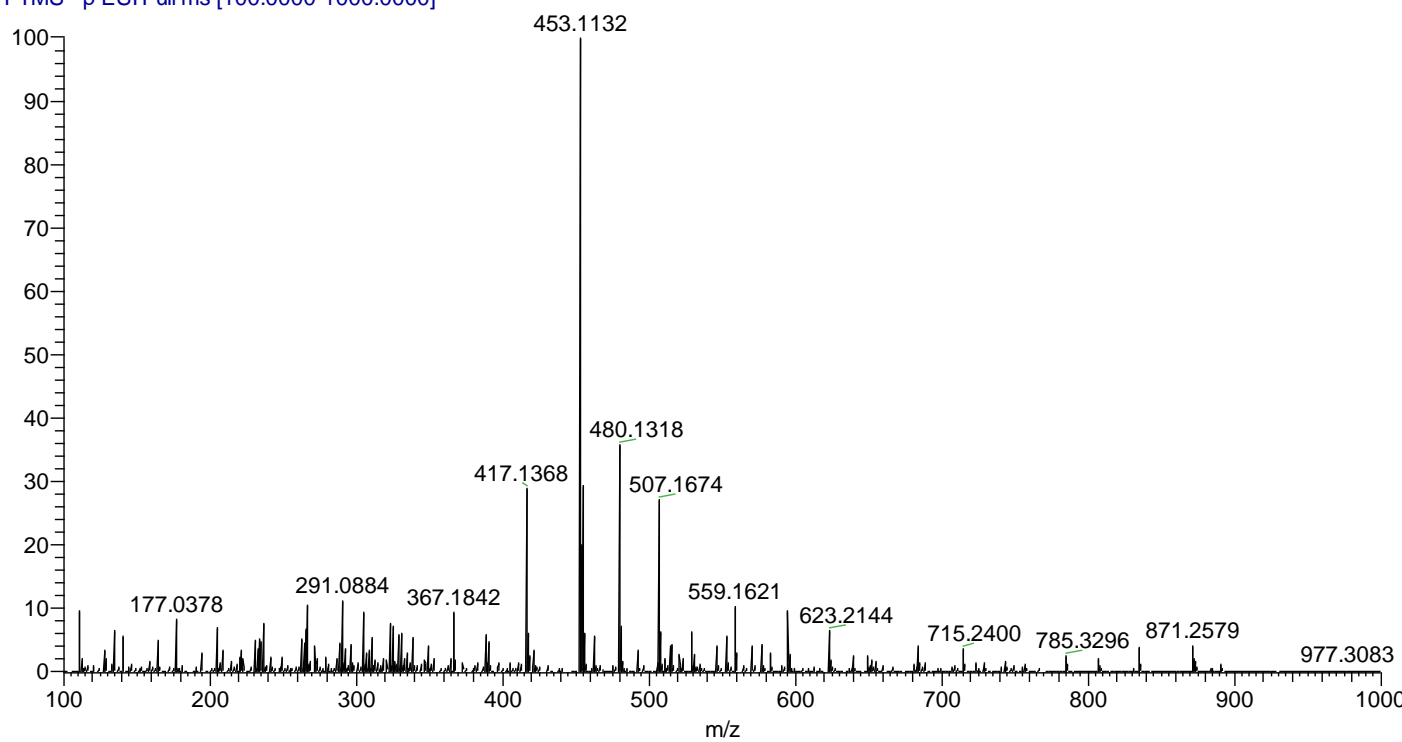

HRESIMS in the negative mode of compound $\mathbf{1 0}$

FH27 \#122 RT: 0.35 AV: 1 NL: 9.07E6

T: FTMS - p ESIFull ms2 417.1391@hcd25.00 [!

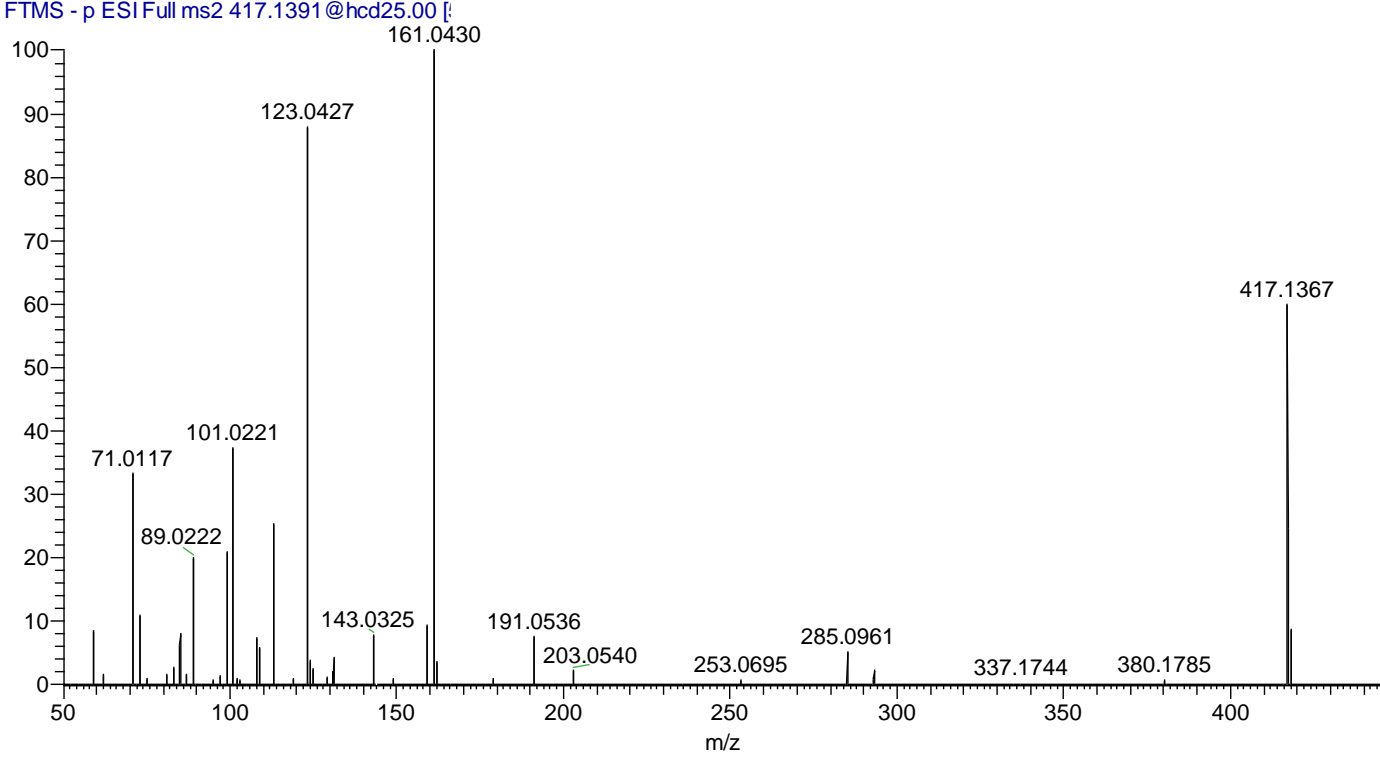

MS/MS in the negative mode of compound $\mathbf{1 0}$

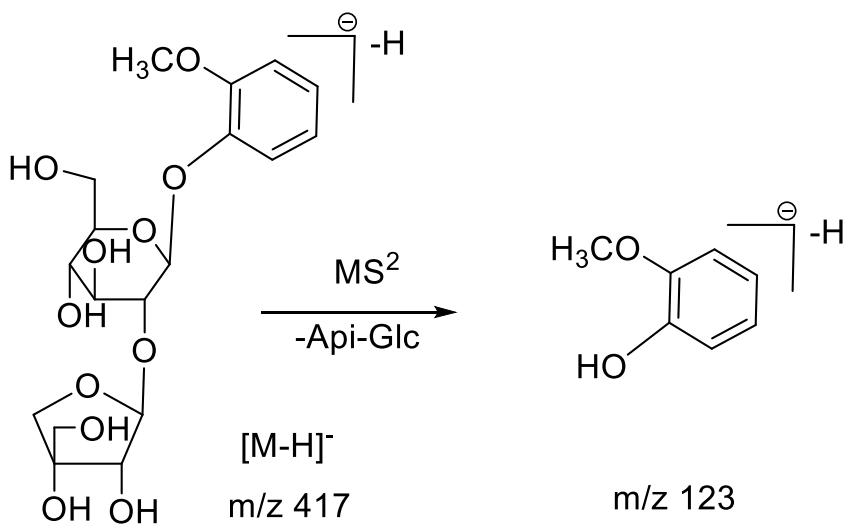

Proposed fragmentation of compound $\mathbf{1 0}$ 
FH37 \#133 RT: 0.38 AV: 1 NL: 2.66E8

T: FTMS - p ESI Full ms [100.0000-1000.0000]

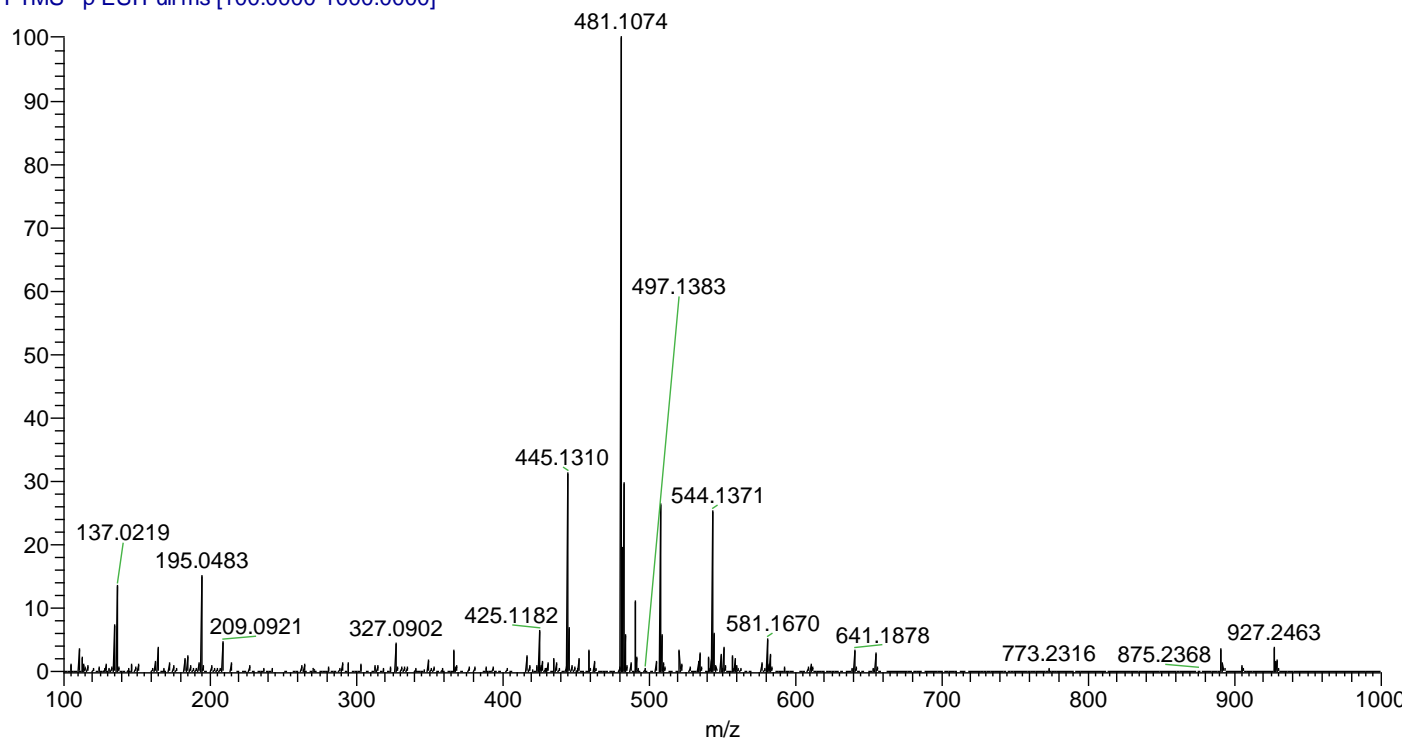

HRESIMS in the negative mode of compound $\mathbf{1 1}$

FH37 \#120 RT: 0.35 AV: 1 NL: $2.98 E 7$

T: FTMS - p ESI Full ms2 445.1341@hcd25.00 [:

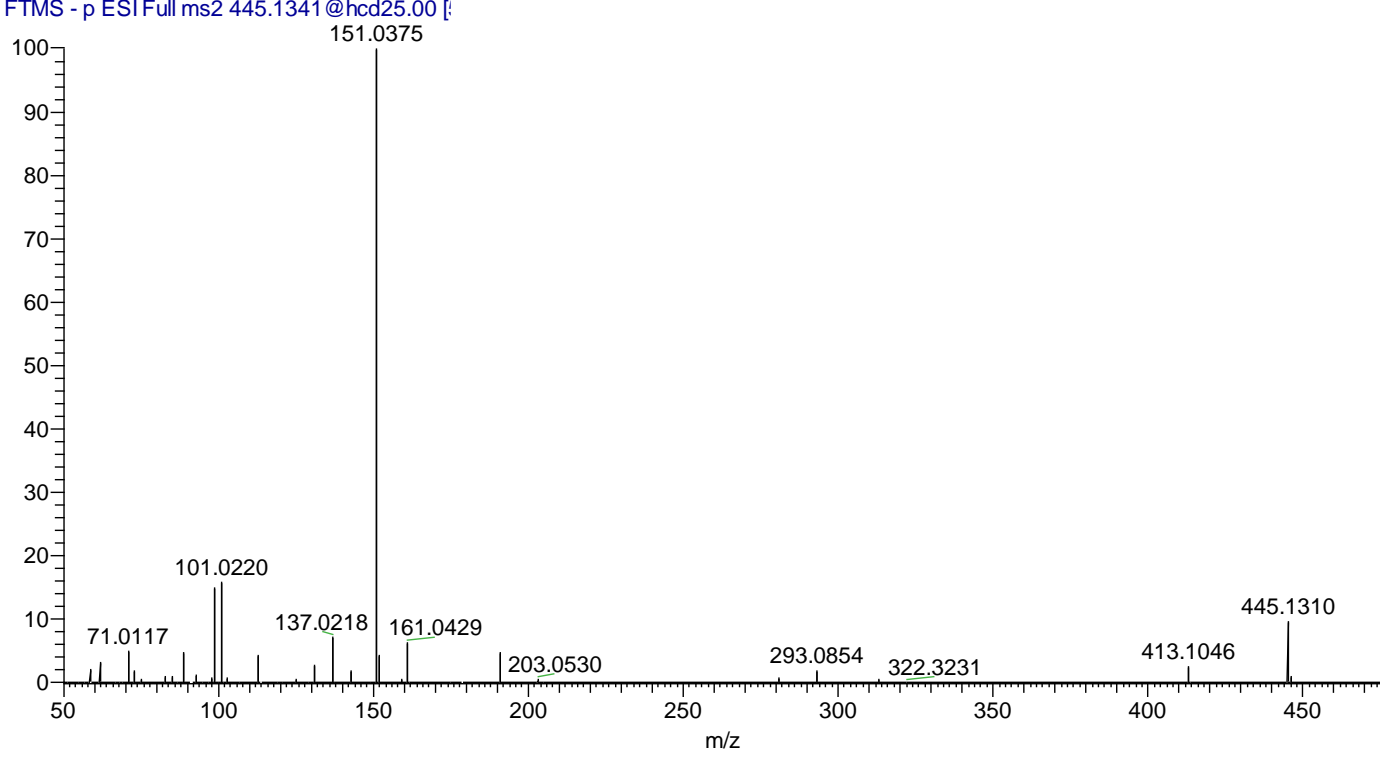

MS/MS in the negative mode of compound $\mathbf{1 1}$

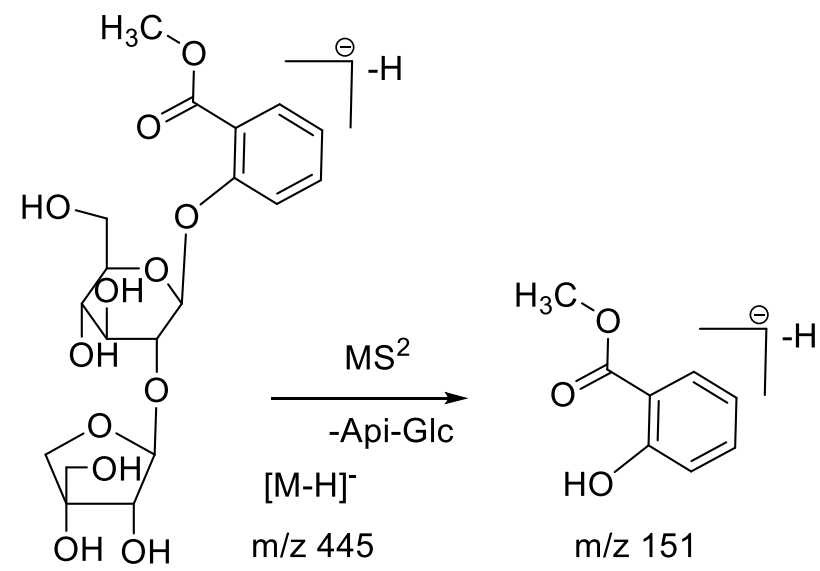

Proposed fragmentation of compound $\mathbf{1 1}$ 
FH65 \#111 RT: 0.32 AV: 1 NL: 2.44E9

T: FTMS - p ESI Full ms [100.0000-1000.0000]

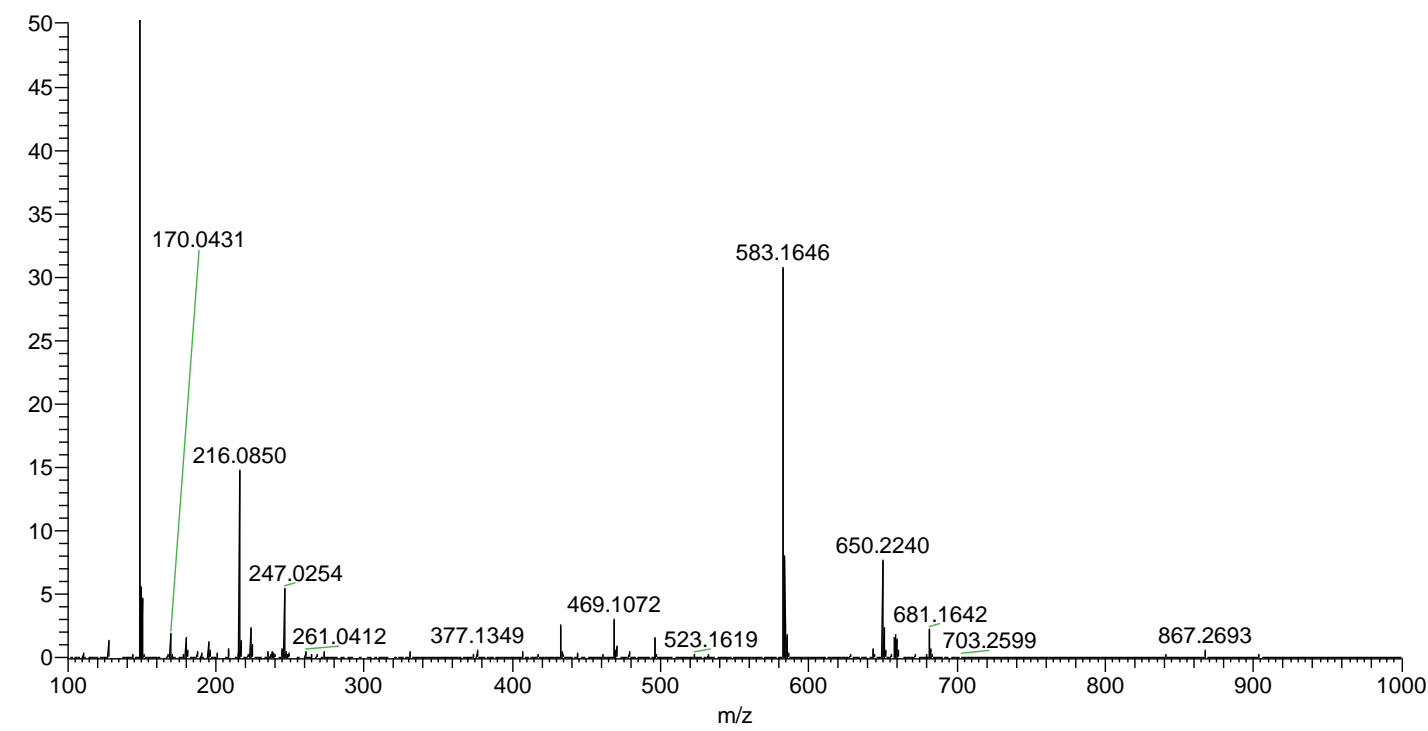

HRESIMS in the negative mode of compound $\mathbf{1 2}$

FH65 \#112 RT: 0.33 AV: 1 NL: 4.69E6

T: FTMS - p ESI Full ms2 433.1341@hcd25.00 [!

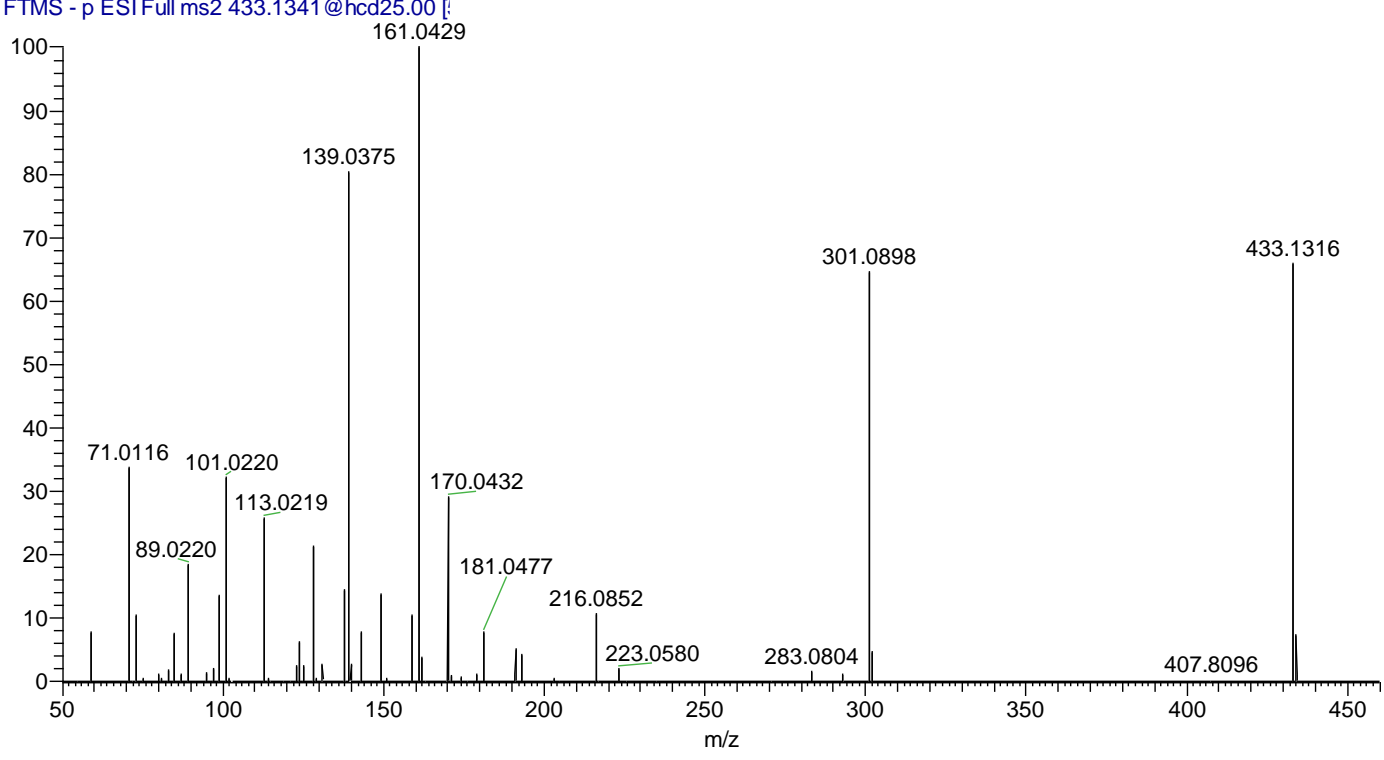

MS/MS in the negative mode of compound $\mathbf{1 2}$

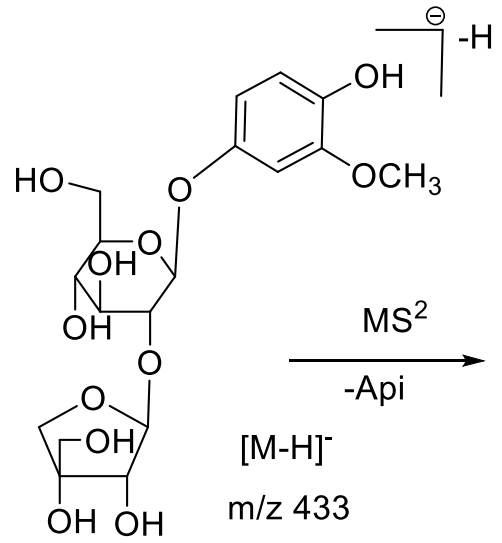<smiles>COc1cc(OC2OC3(CO)OC(O)C(O)C2O3)ccc1O</smiles>

Proposed fragmentation of compound $\mathbf{1 2}$ 


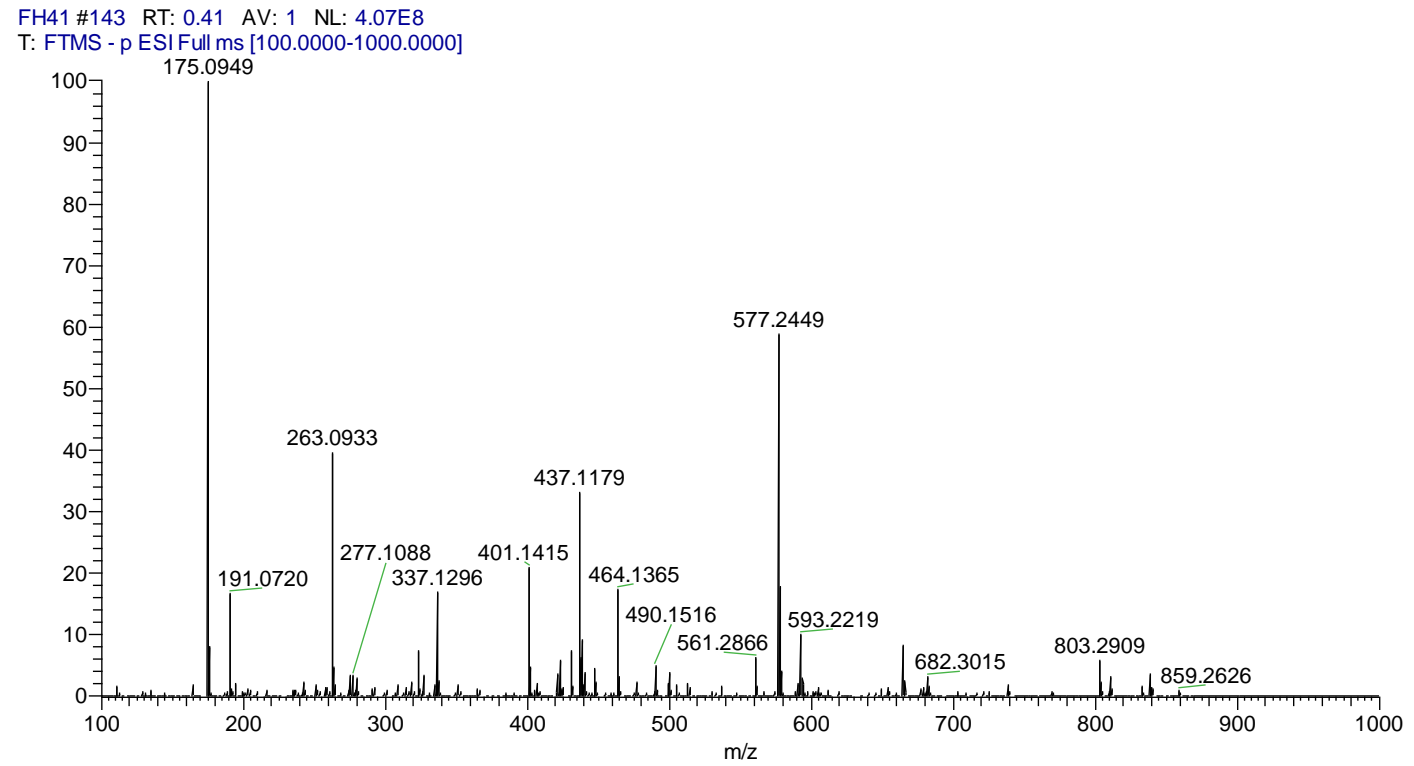

HRESIMS in the negative mode of compound $\mathbf{1 3}$

FH41 \#142 RT: 0.41 AV: 1 NL: 1.01E7

T: FTMS - p ESI Full ms2 401.1442@hcd25.00 [

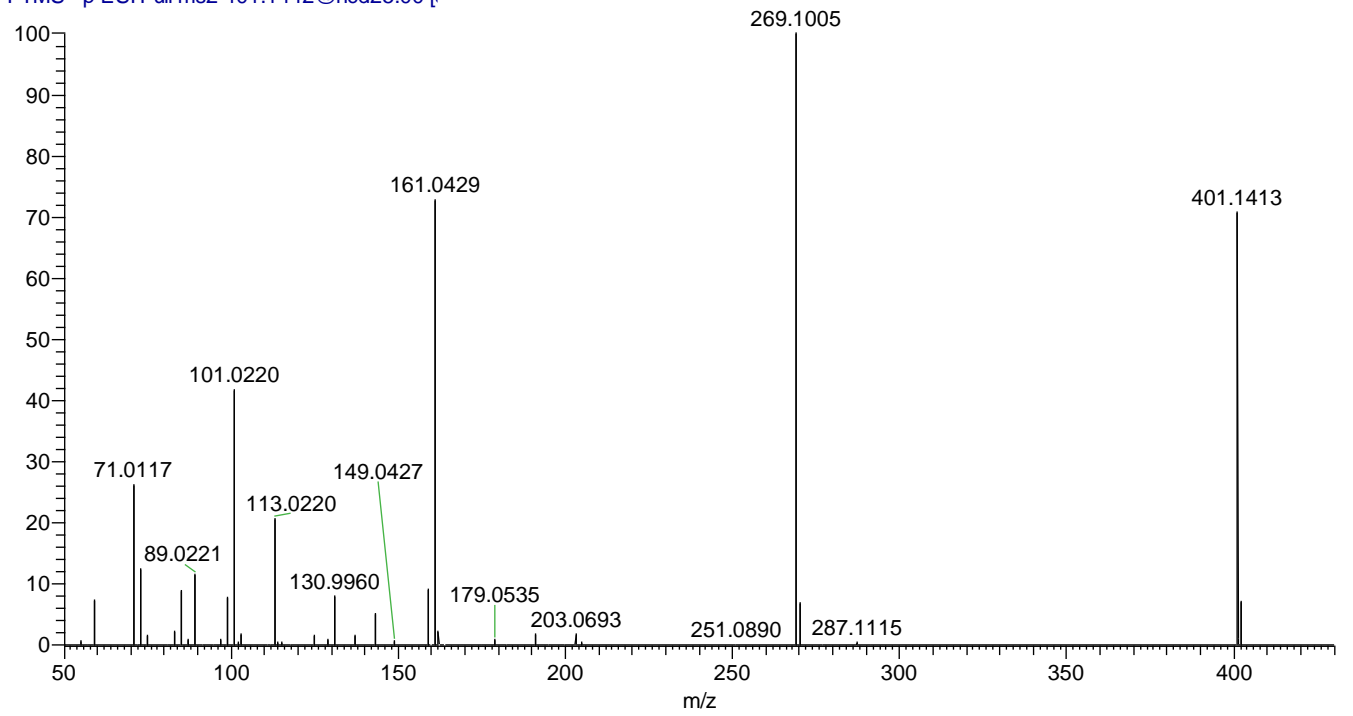

MS/MS in the negative mode of compound $\mathbf{1 3}$

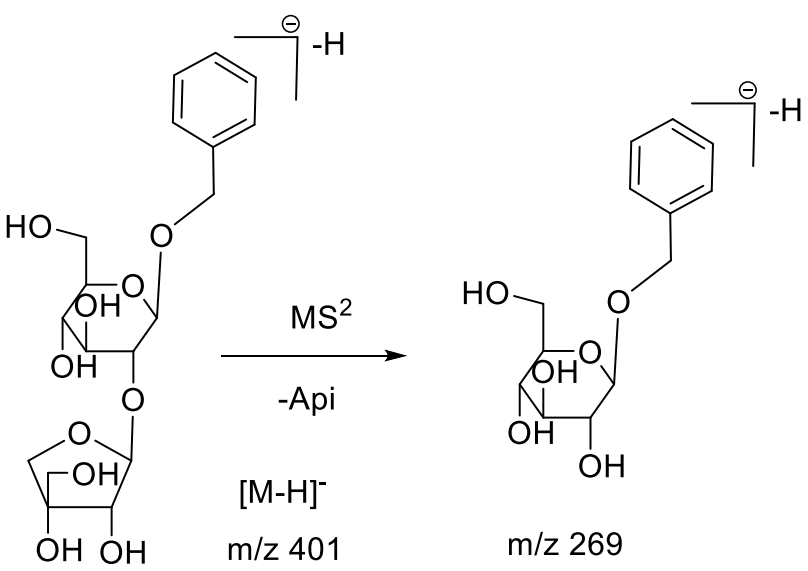

Proposed fragmentation of compound $\mathbf{1 3}$ 
FH31 \#121 RT: 0.35 AV: 1 NL: 5.88E8

T: FTMS - p ESI Full ms [100.0000-1000.0000

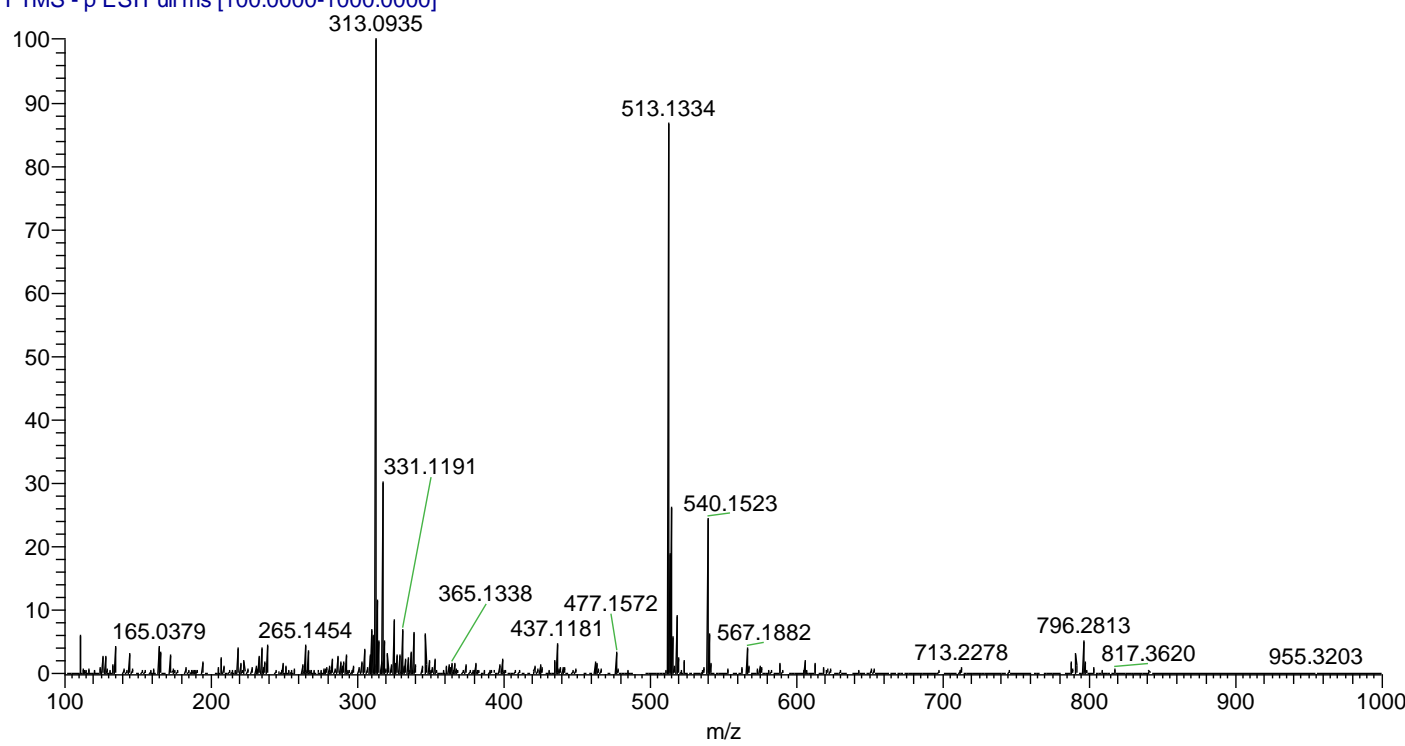

HRESIMS in the negative mode of compound 14

FH31 \#124 RT: 0.36 AV: 1 NL: 4.03E6

T: FTMS - p ESIFull ms2 477.1603@hcd25.00 [!

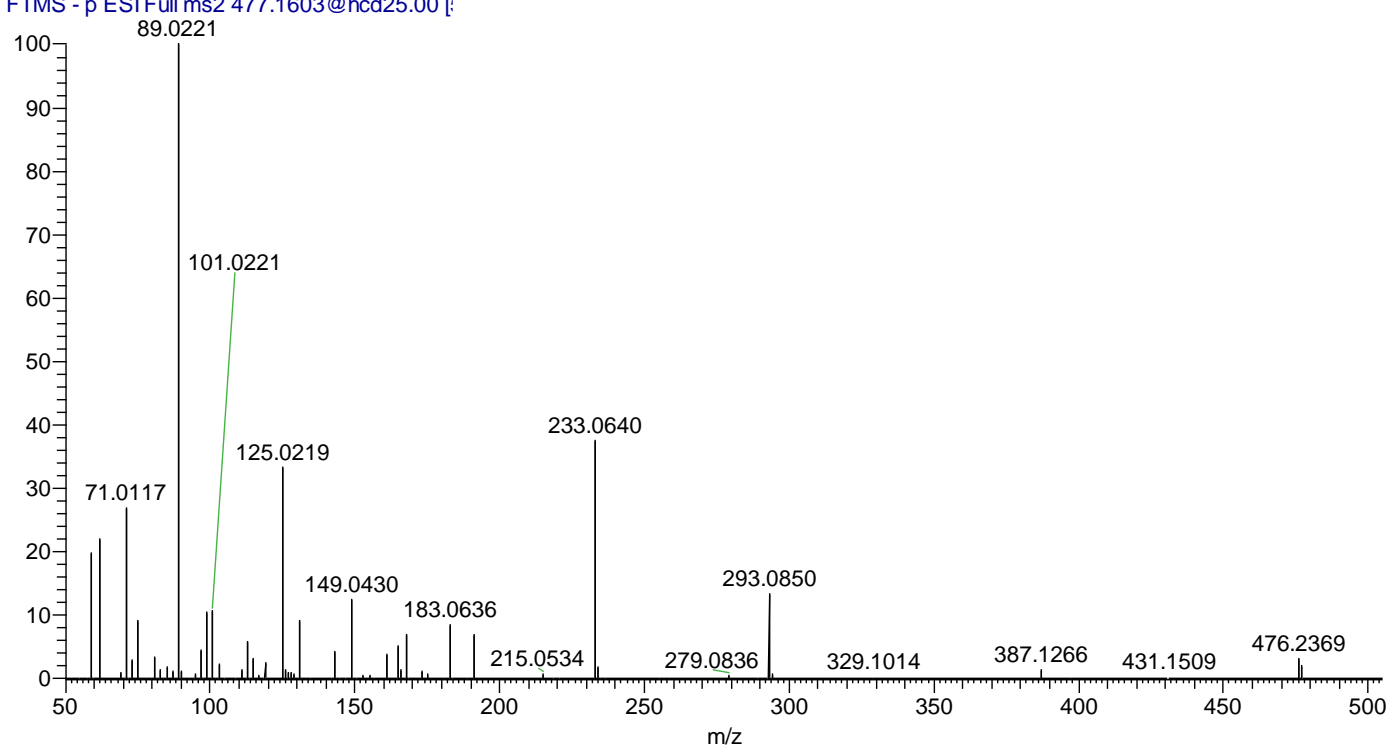

MS/MS in the negative mode of compound 14<smiles>COc1cc(OC2C3OC(OCC(O)C(O)C(O)C(O)COc4c(OC)cc(O)cc4OC)C(O3)C2O)cc(OC)c1OC</smiles>

Proposed fragmentation of compound $\mathbf{1 4}$ 


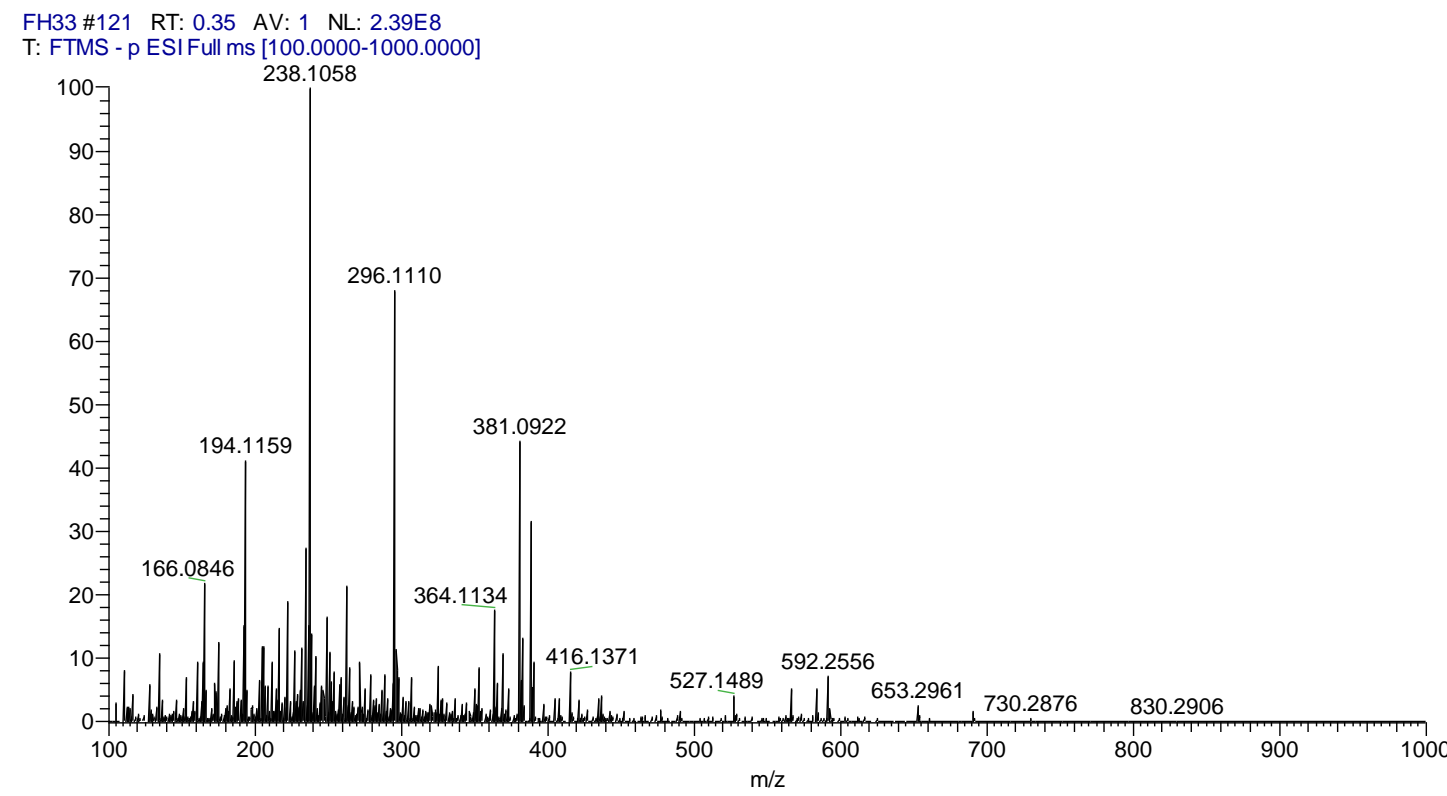

HRESIMS in the negative mode of compound $\mathbf{1 5}$

FH33 \#120 RT: 0.35 AV: 1 NL: $1.82 E 6$

T: FTMS - p ESI Full ms2 345.1180@hcd25.00 [!

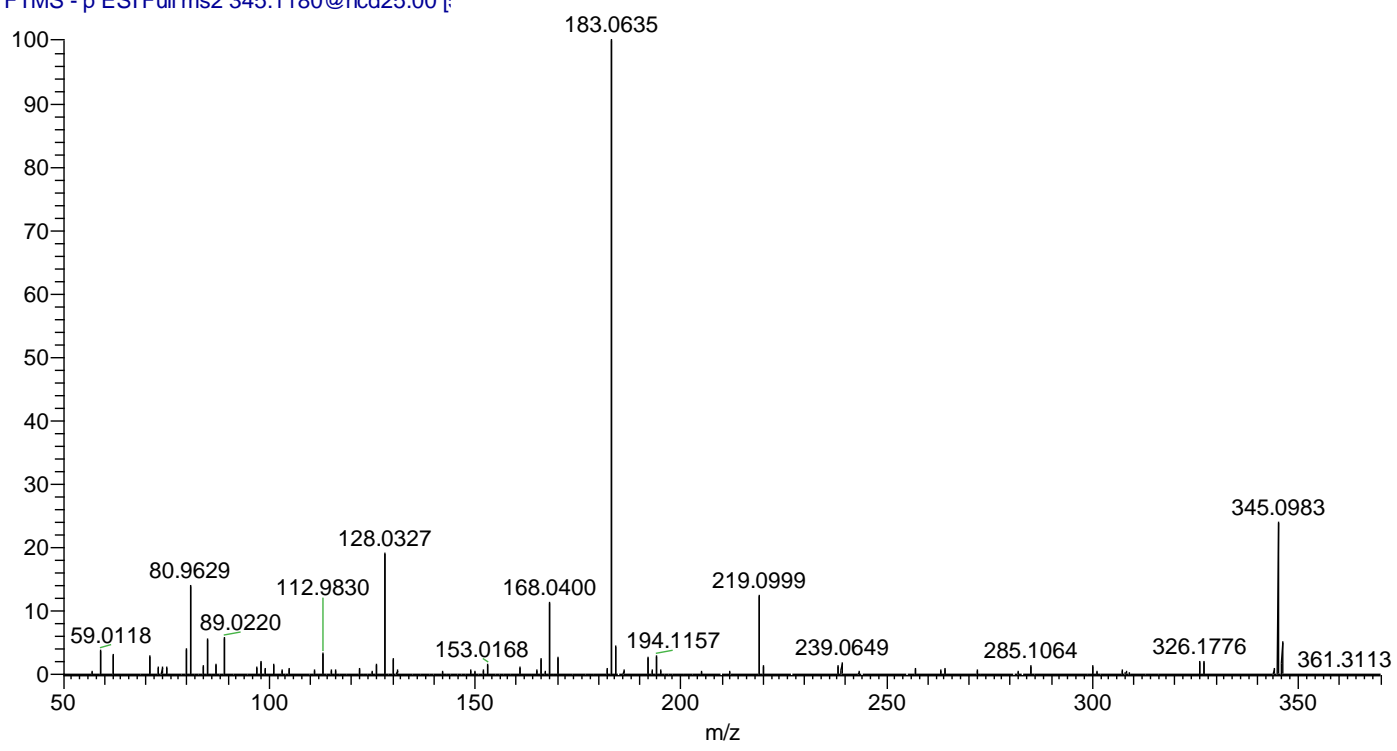

MS/MS in the negative mode of compound $\mathbf{1 5}$<smiles>COc1cc(CO)cc(OC)c1OCC(C)O</smiles>

Proposed fragmentation of compound $\mathbf{1 5}$ 


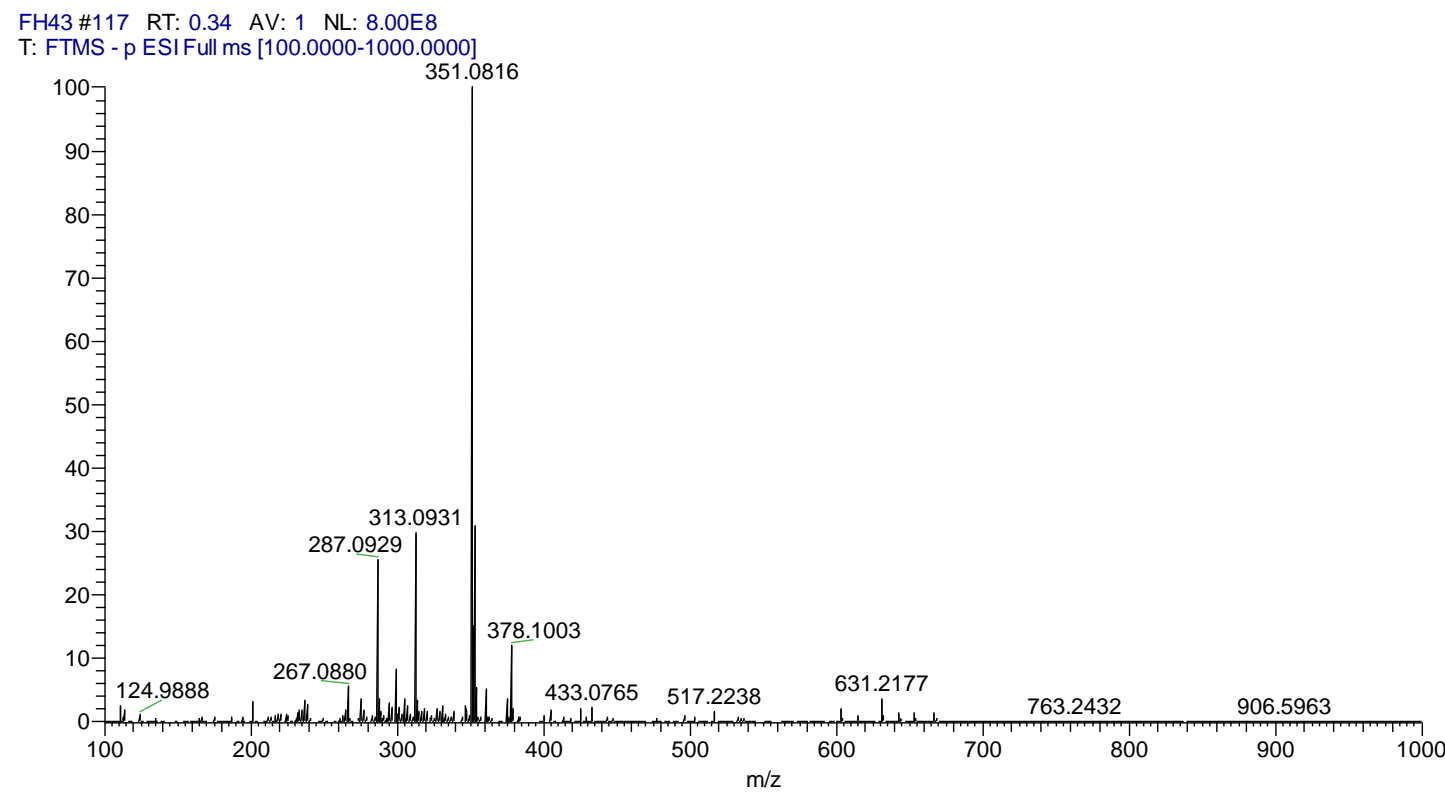

HRESIMS in the negative mode of compound $\mathbf{1 6}$

FH43 \#118 RT: 0.34 AV: 1 NL: 4.57E6

T: FTMS - p ESI Full ms2 315.1074@hcd25.00 [!

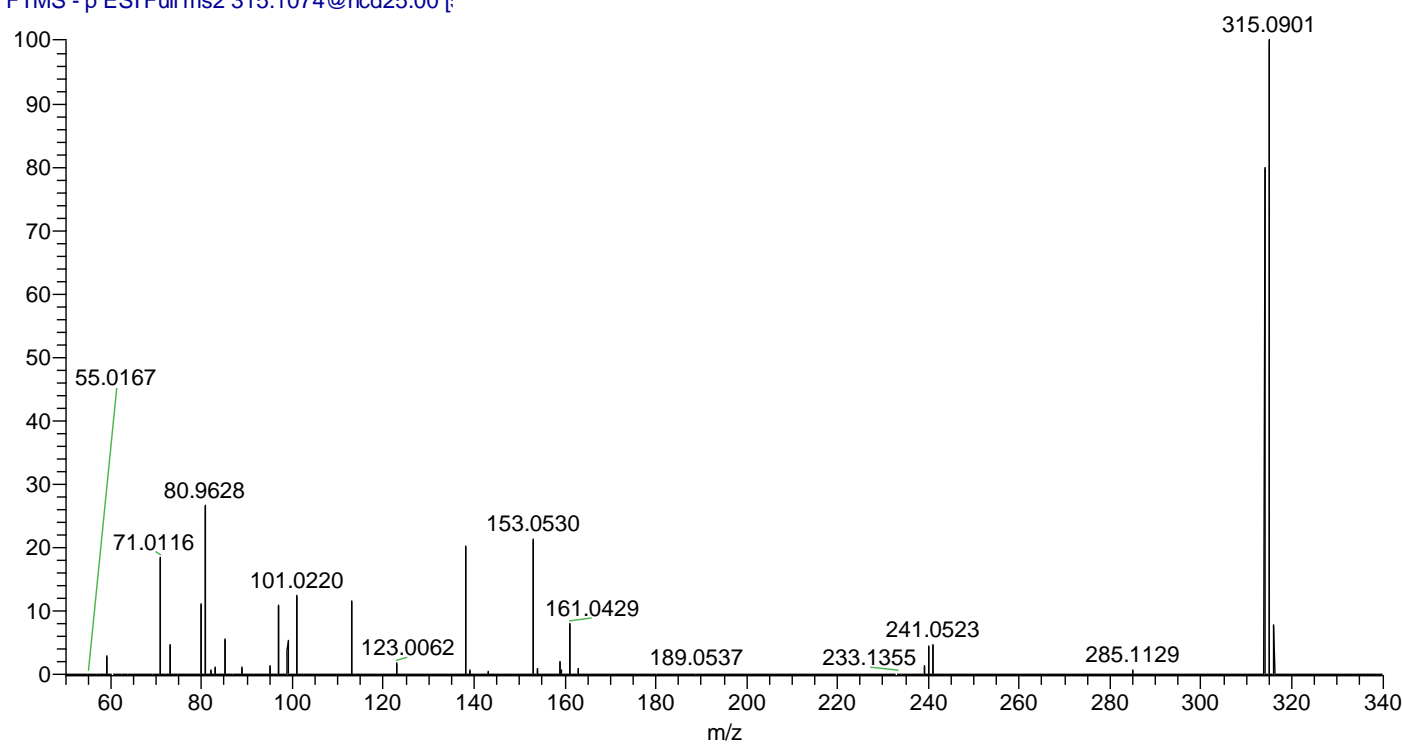

HRESIMS in the negative mode of compound $\mathbf{1 6}$

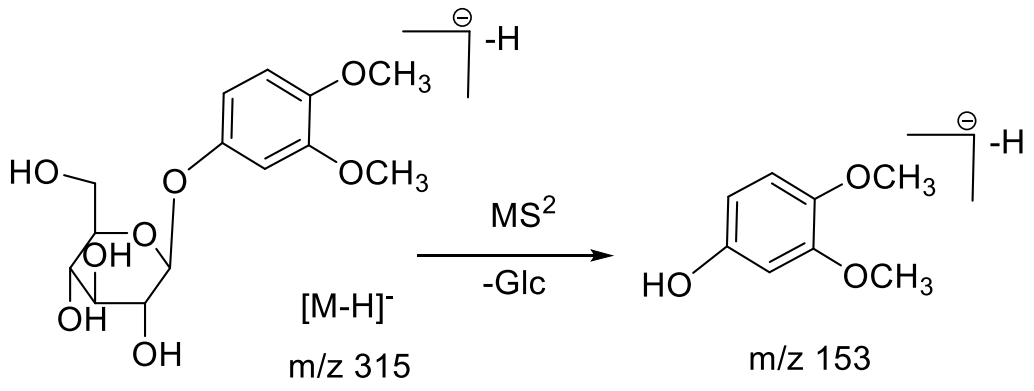

Proposed fragmentation of compound $\mathbf{1 6}$ 
FH45 \#121 RT: 0.35 AV: 1 NL: 1.24E9

T: FTMS - p ESI Full ms [100.0000-1000.0000]

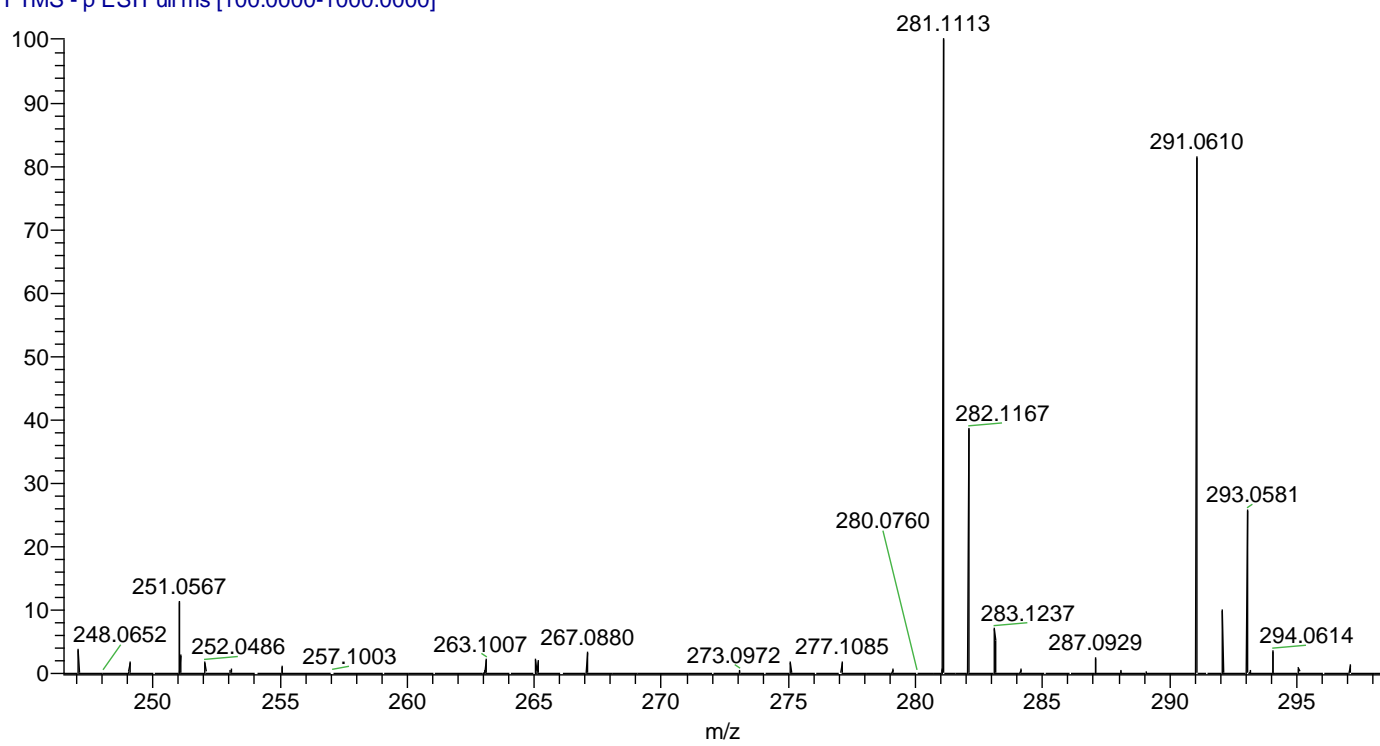

HRESIMS in the negative mode of compound $\mathbf{1 7}$

FH45 \#108 RT: 0.31 AV: 1 NL: $8.38 E 5$

T: FTMS - p ESI Full ms2 255.0863@hcd25.00 [!

$255.0863 @$ hcd
93.0322

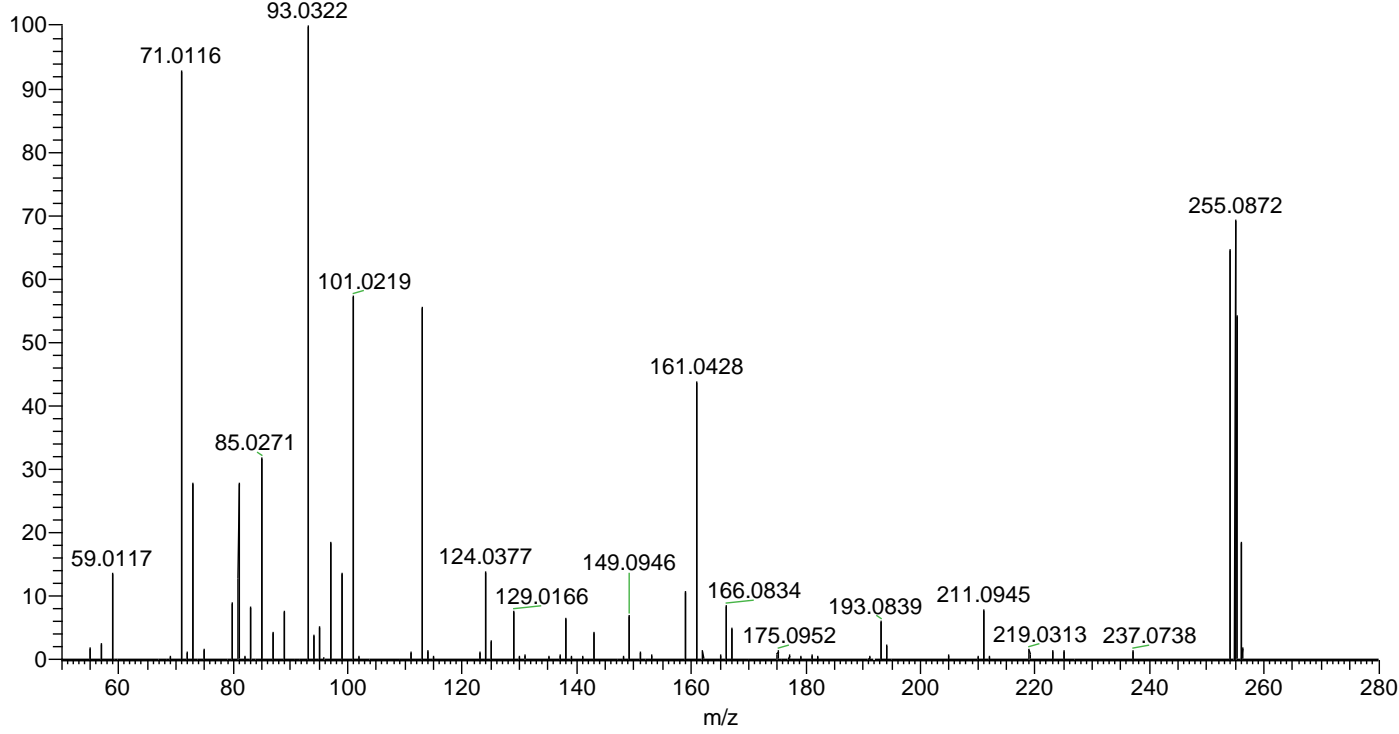

MS/MS in the negative mode of compound $\mathbf{1 7}$

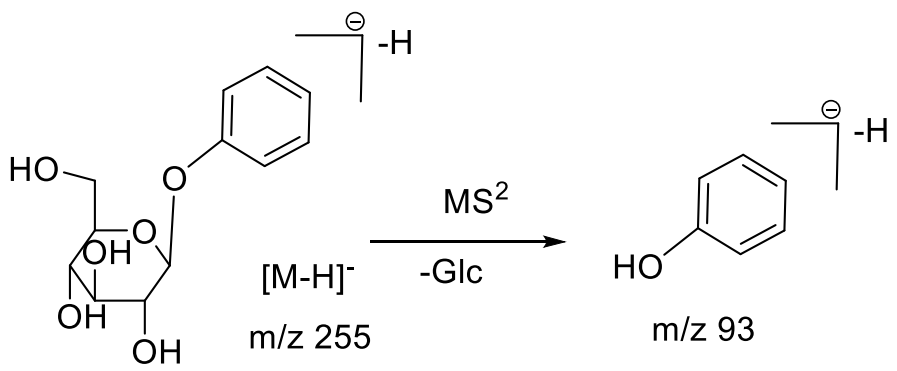

Proposed fragmentation of compound $\mathbf{1 7}$ 
FH46 \#125 RT: 0.36 AV: 1 NL: 4.11E8

T: FTMS - p ESI Full ms [100.0000-1000.0000]

100 每

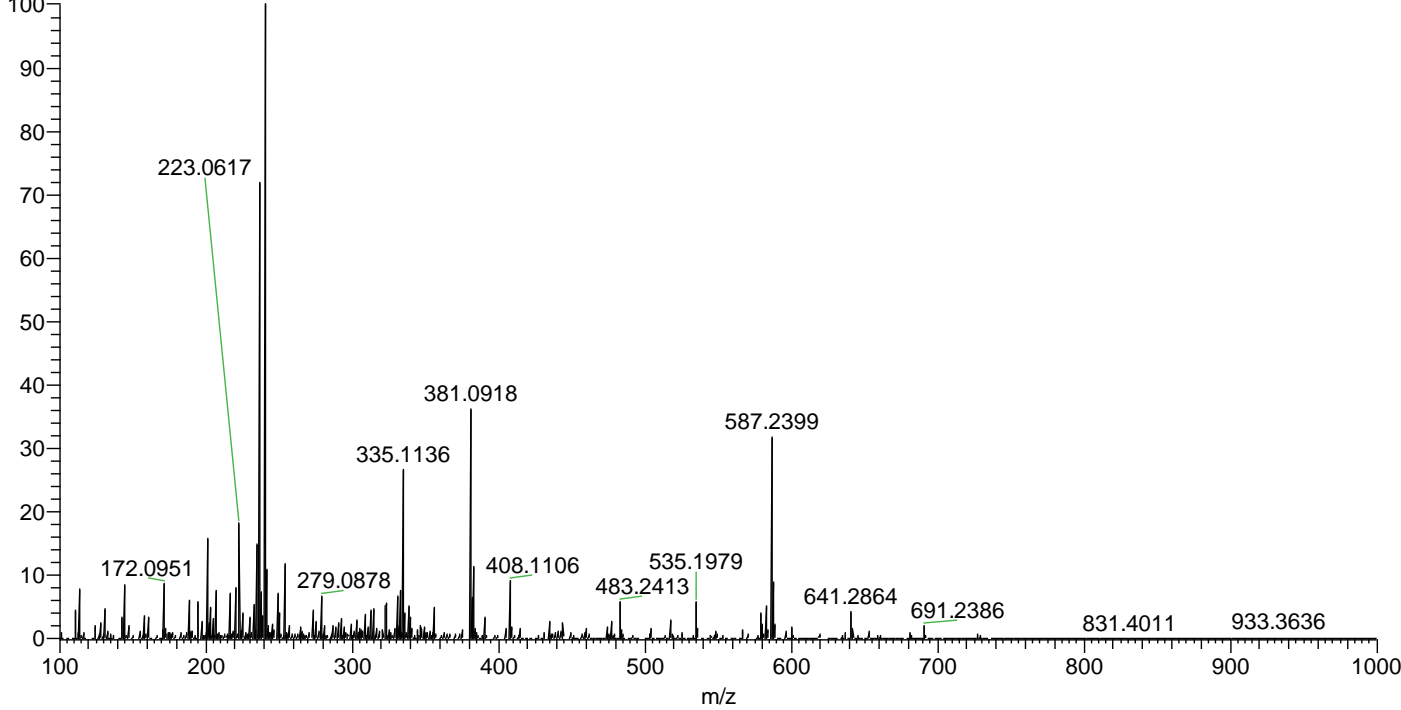

HRESIMS in the negative mode of compound $\mathbf{1 8}$

FH46 \#114 RT: 0.33 AV: 1 NL: 1.15E6

T: FTMS - p ESI Full ms2 345.1180@hcd25.00 [!

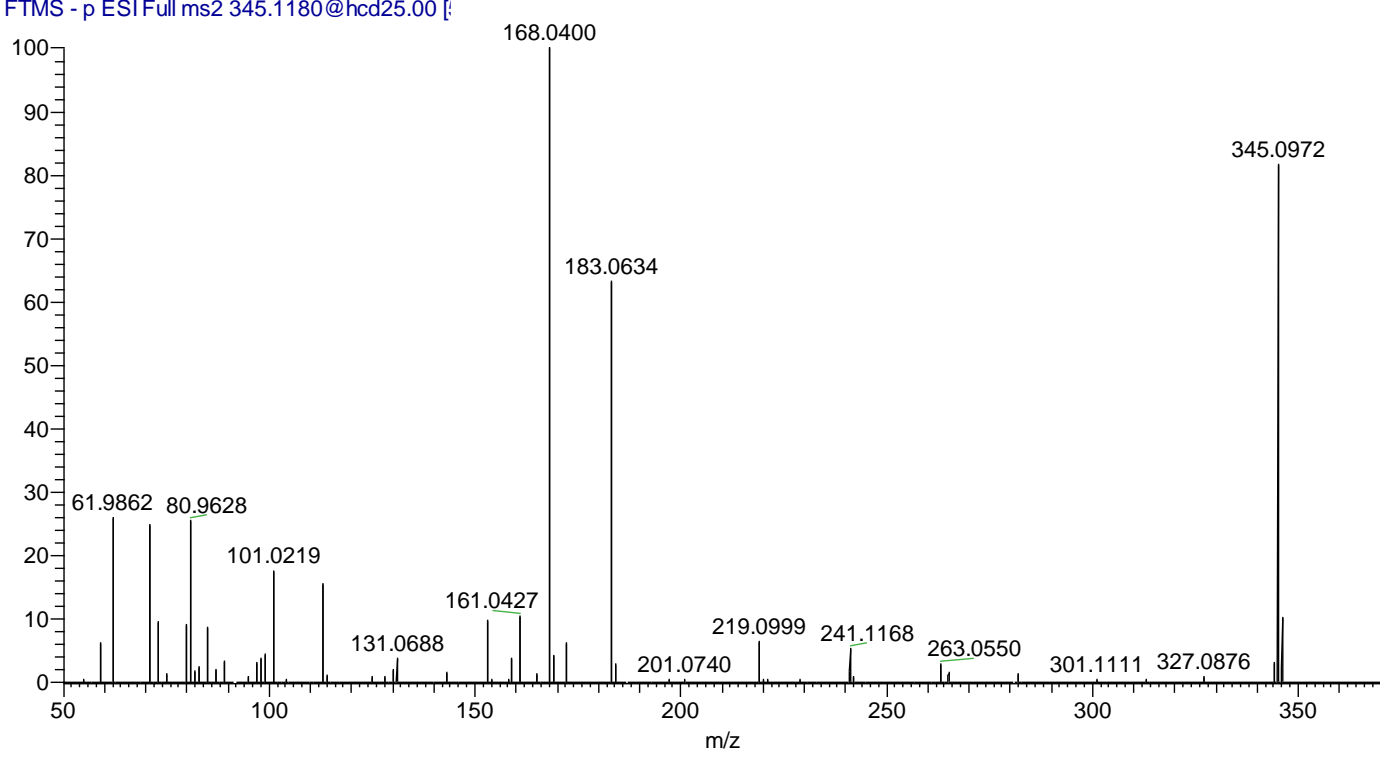

MS/MS in the negative mode of compound $\mathbf{1 8}$<smiles>COc1cc(OC)c(OC2C(O)C(O)C(O)C2Oc2c(OC)cc(OC)c(OC)c2OC)c(OC)c1</smiles>

Proposed fragmentation of compound $\mathbf{1 8}$ 


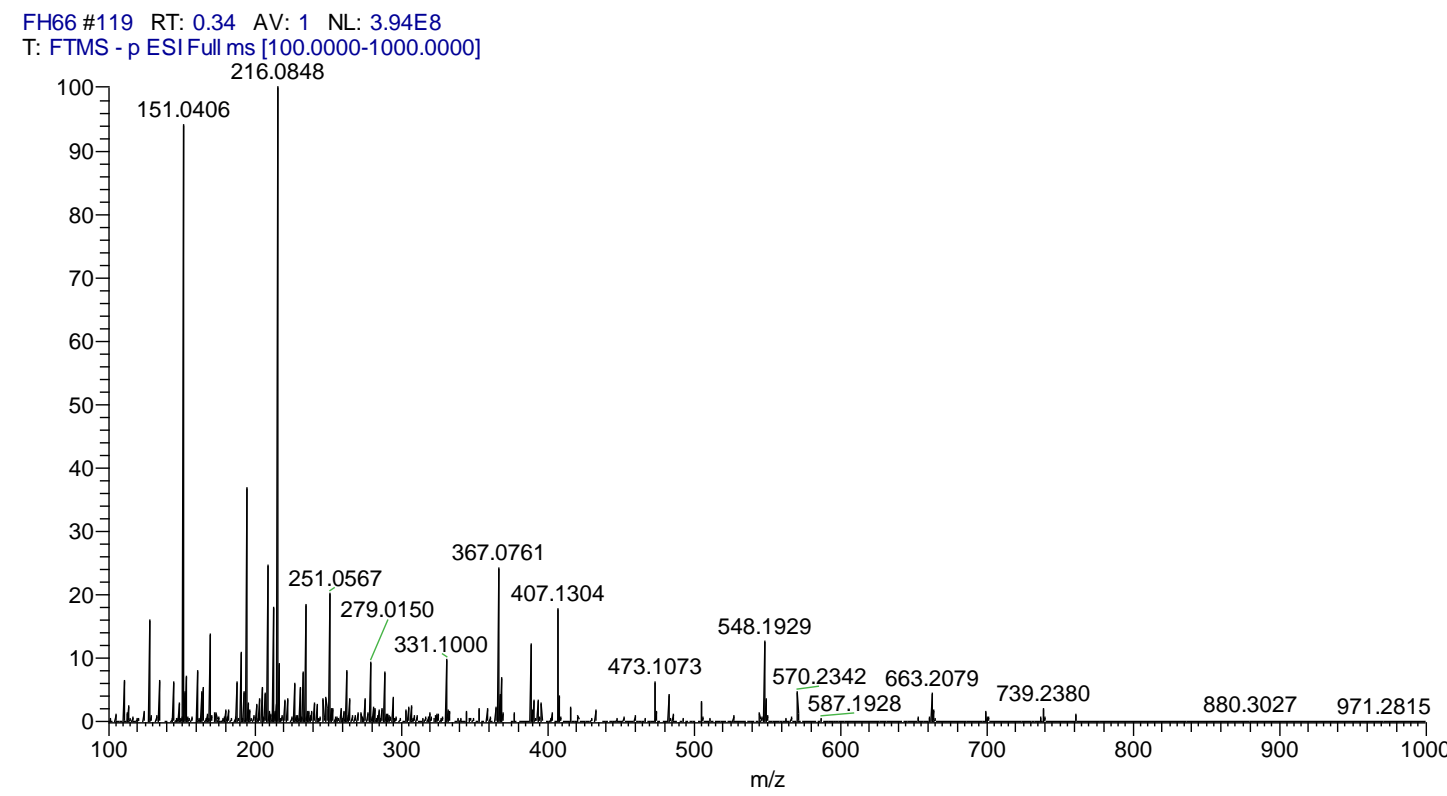

HRESIMS in the negative mode of compound 19

FH66 \#120 RT: 0.35 AV: 1 NL: 9.13E6

T: FTMS - p ESI Full ms2 331.1024@hcd25.00 [!

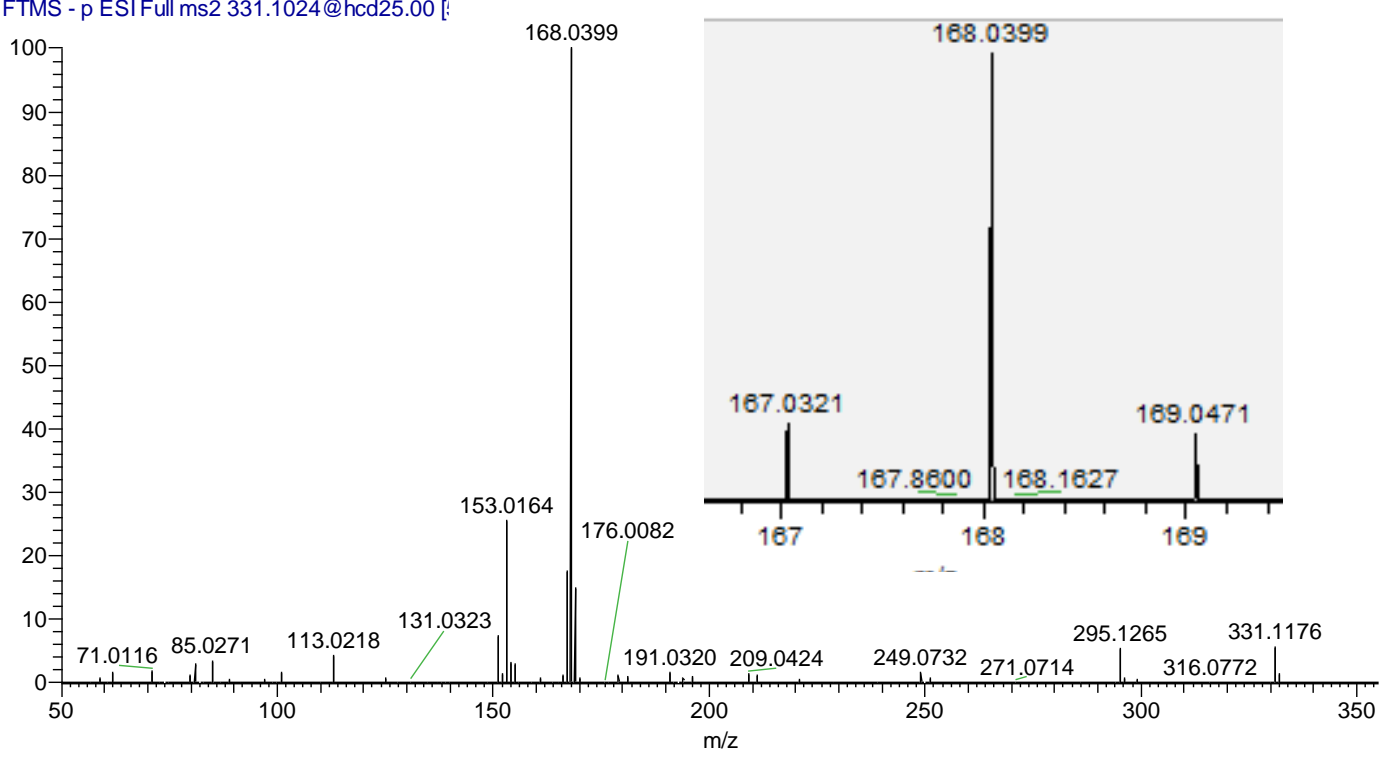

MS/MS in the negative mode of compound 19<smiles>COc1cc(OC2OC(CO)C(O)C(O)C2O)cc(OC)c1O</smiles><smiles>COc1cc(O)cc(OC)c1O</smiles>

Proposed fragmentation of compound 19 


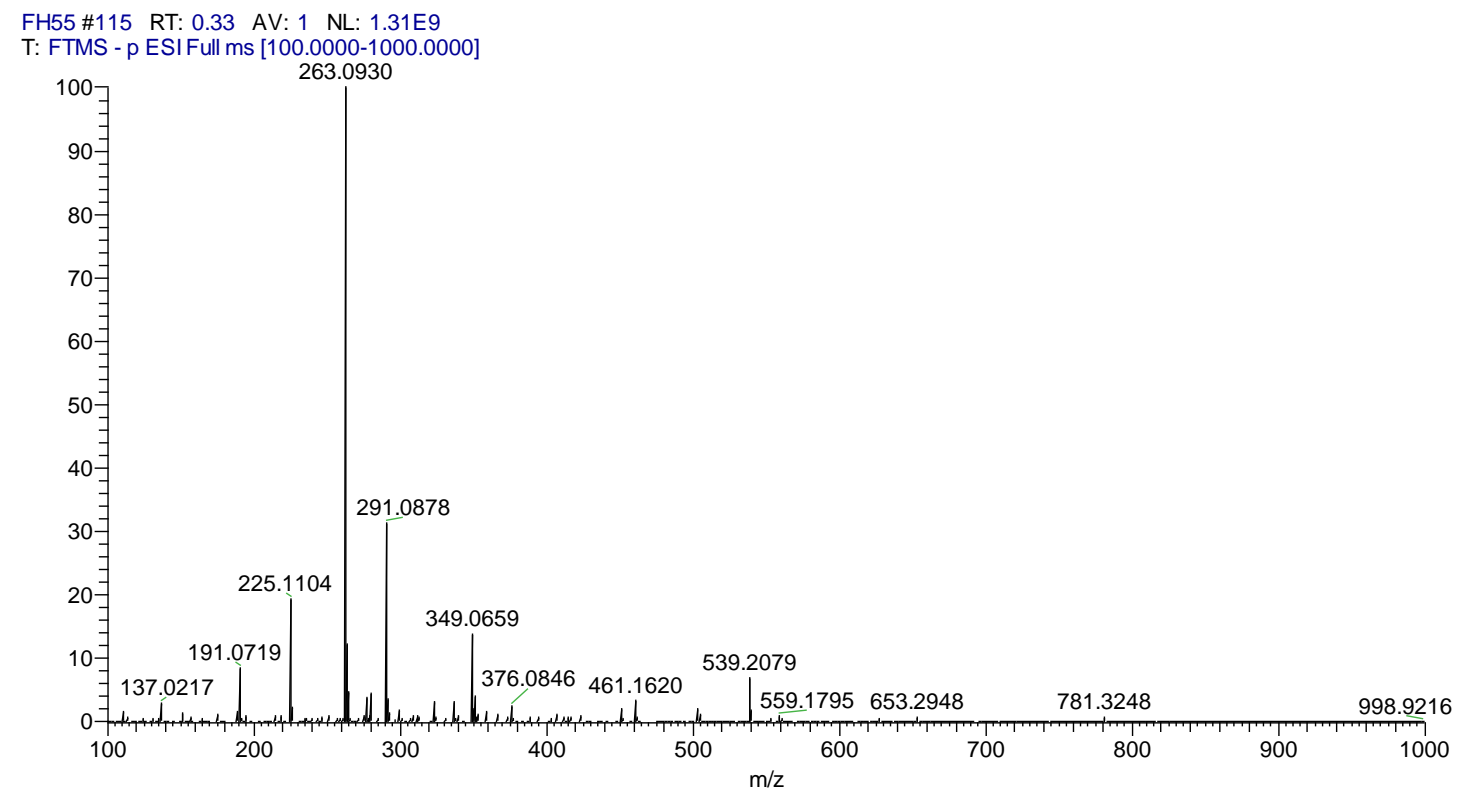

HRESIMS in the negative mode of compound $\mathbf{2 0}$

FH55 \#114 RT: 0.33 AV: 1 NL: 2.26E6

T: FTMS - p ESI Full ms2 313.0918@hcd25.00 [!

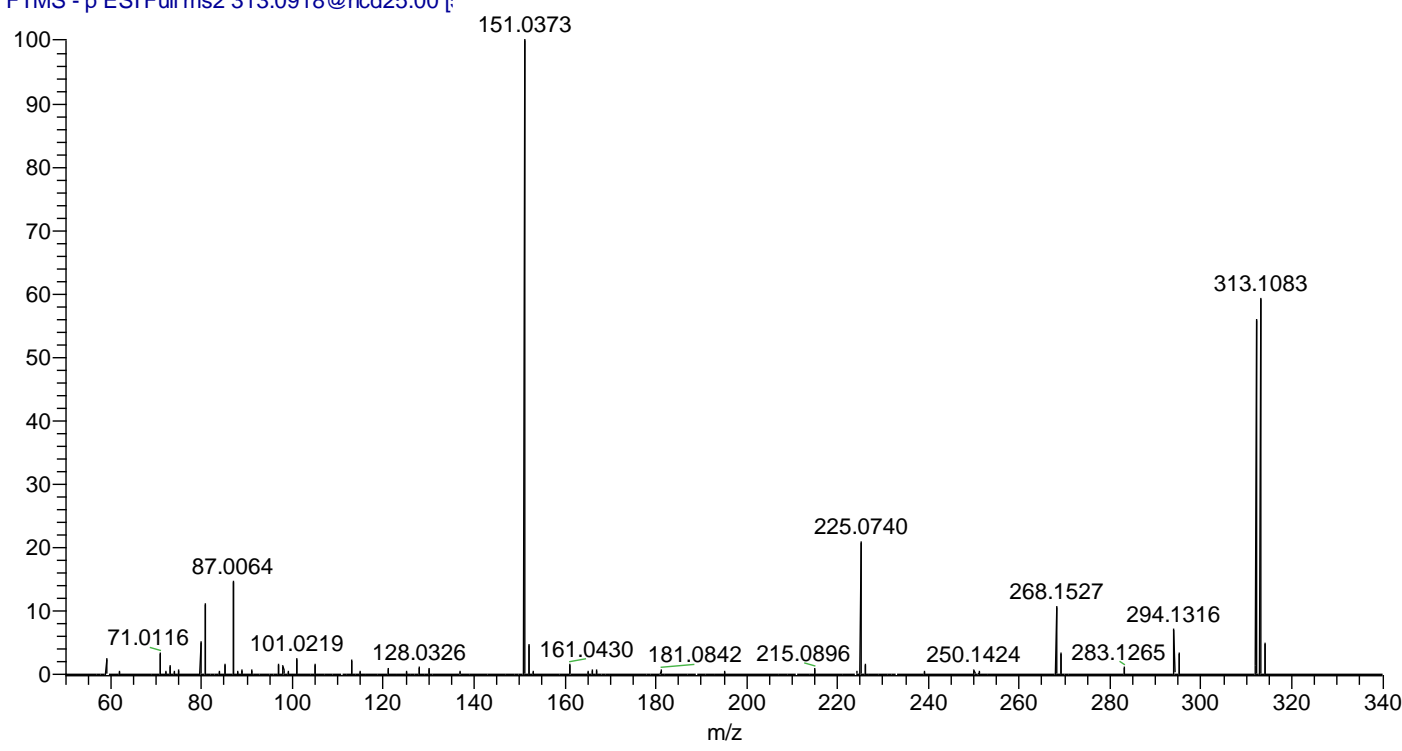

MS/MS in the negative mode of compound $\mathbf{2 0}$<smiles>COC(=O)c1ccccc1OC1OC2(CO)OC1C(O)C(O)C2Oc1ccccc1C(=O)OC</smiles>

Proposed fragmentation of compound $\mathbf{2 0}$ 
FH92 \#103 RT: 0.30 AV: 1 NL: $2.01 \mathrm{Eg}$

T: FTMS - p ESI Full ms [100.0000-1000.0000]
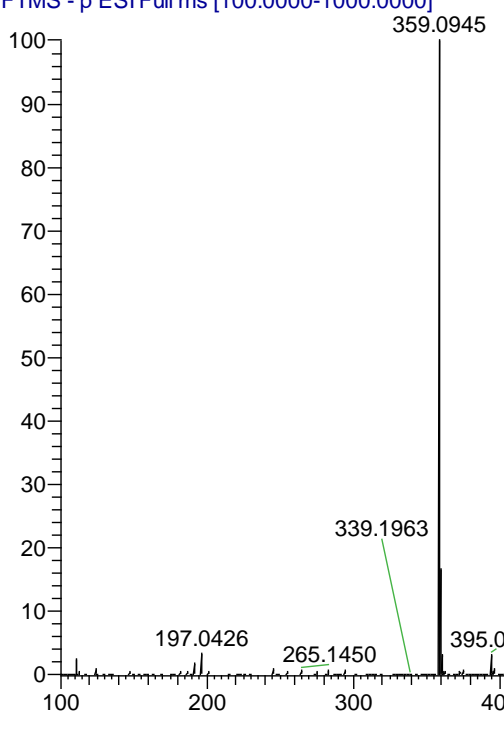

\subsection{5}

HRESIMS in the negative mode of compound $\mathbf{2 1}$

FH92 \#102 RT: 0.30 AV: 1 NL: 5.95E8

T: FTMS - p ESI Full ms2 359.1337@hcd25.00 [!

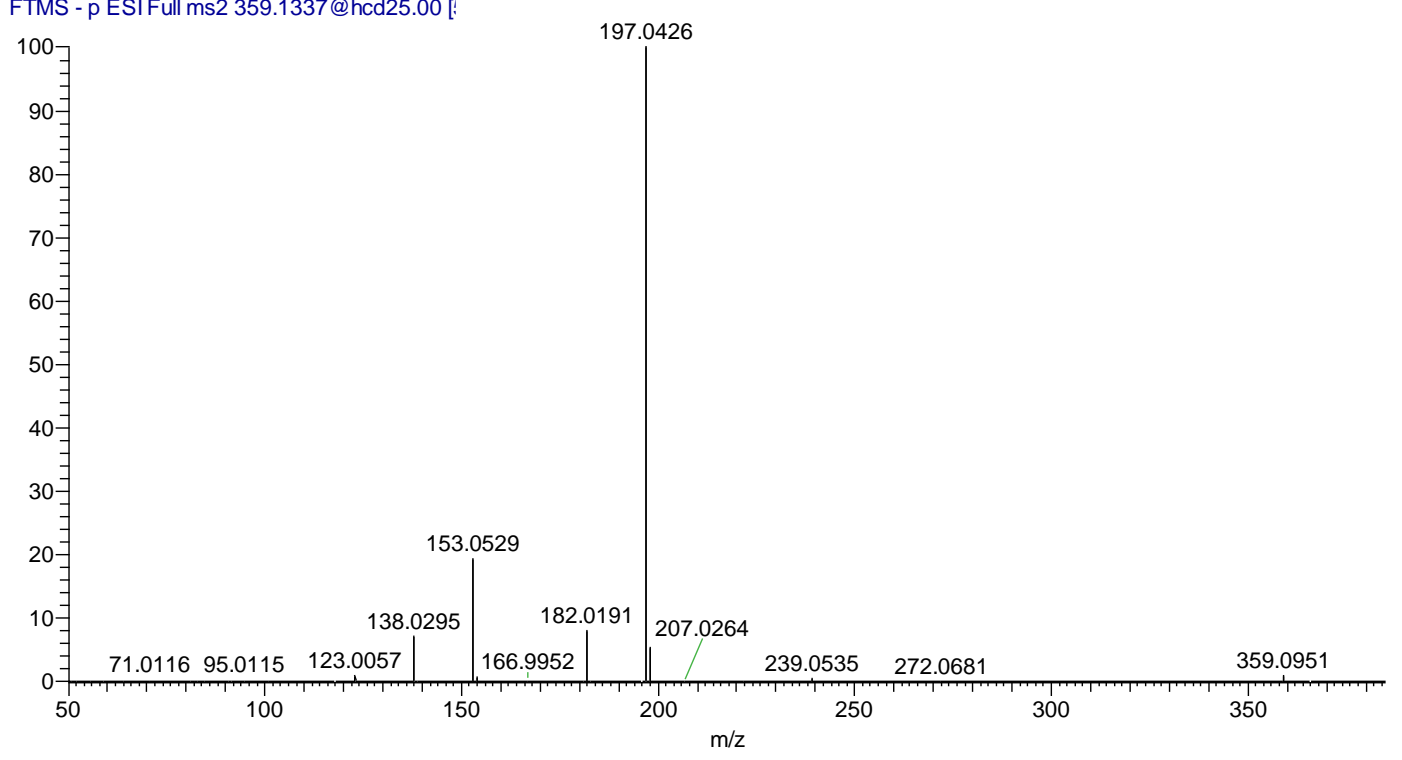

MS/MS in the negative mode of compound 21

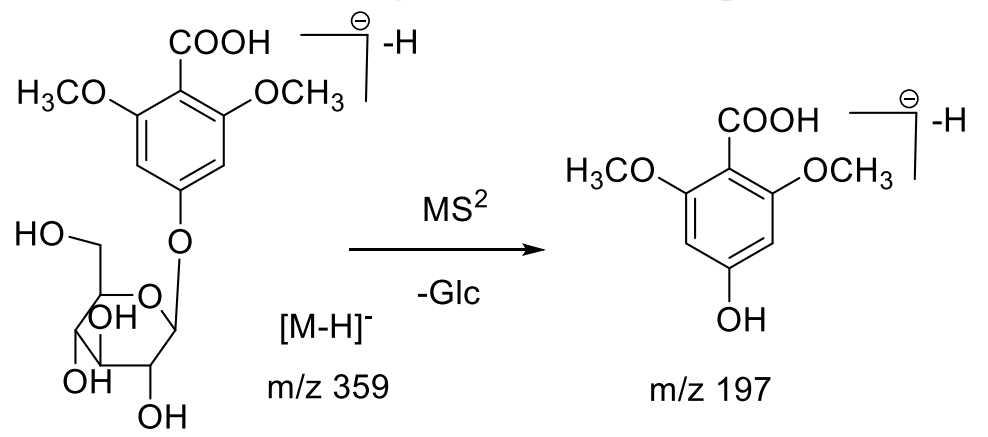

Proposed fragmentation of compound 21 


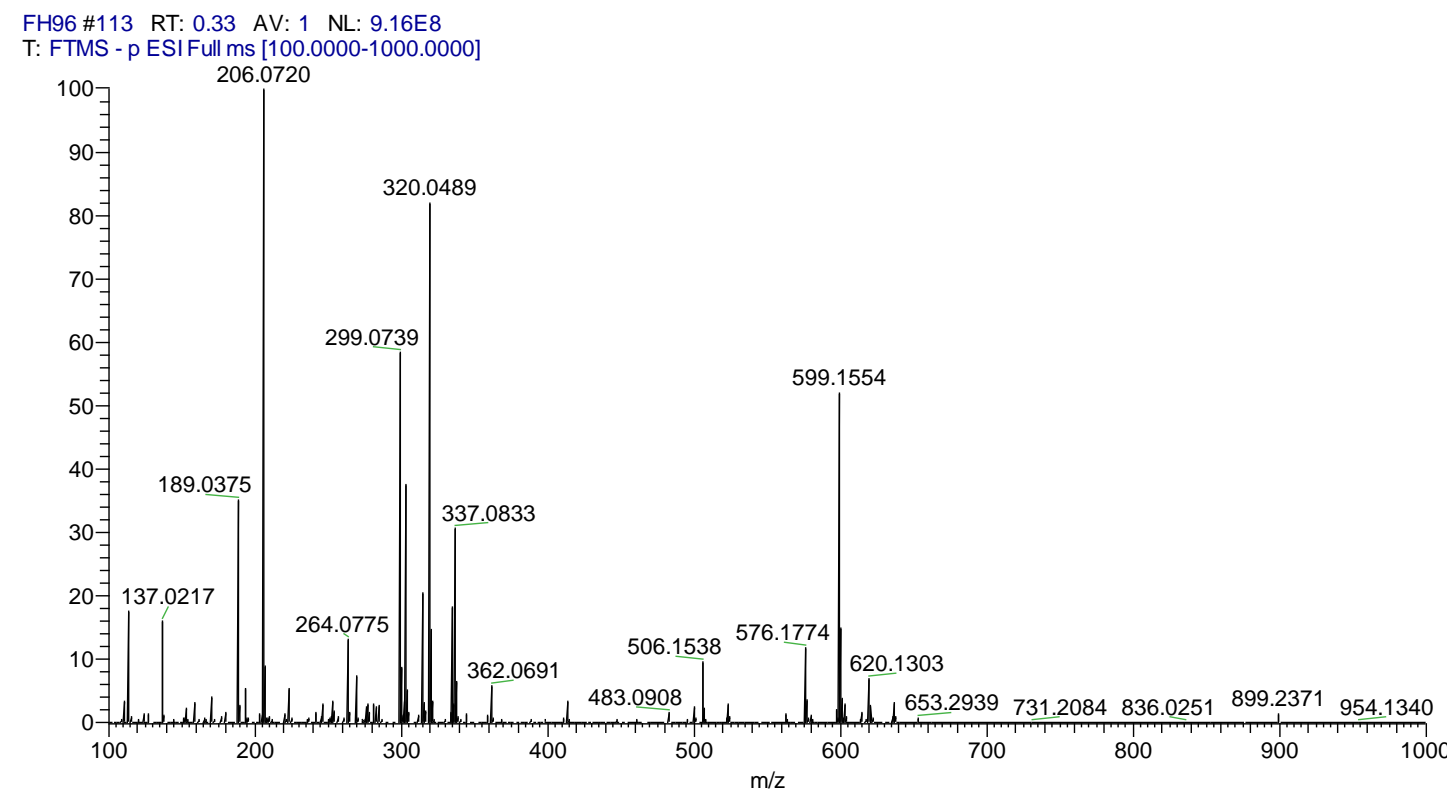

HRESIMS in the negative mode of compound 22

FH96 \#114 RT: 0.33 AV: 1 NL: 2.28E8

T: FTMS - p ESI Full ms2 299.0761@hcd25.00 [!

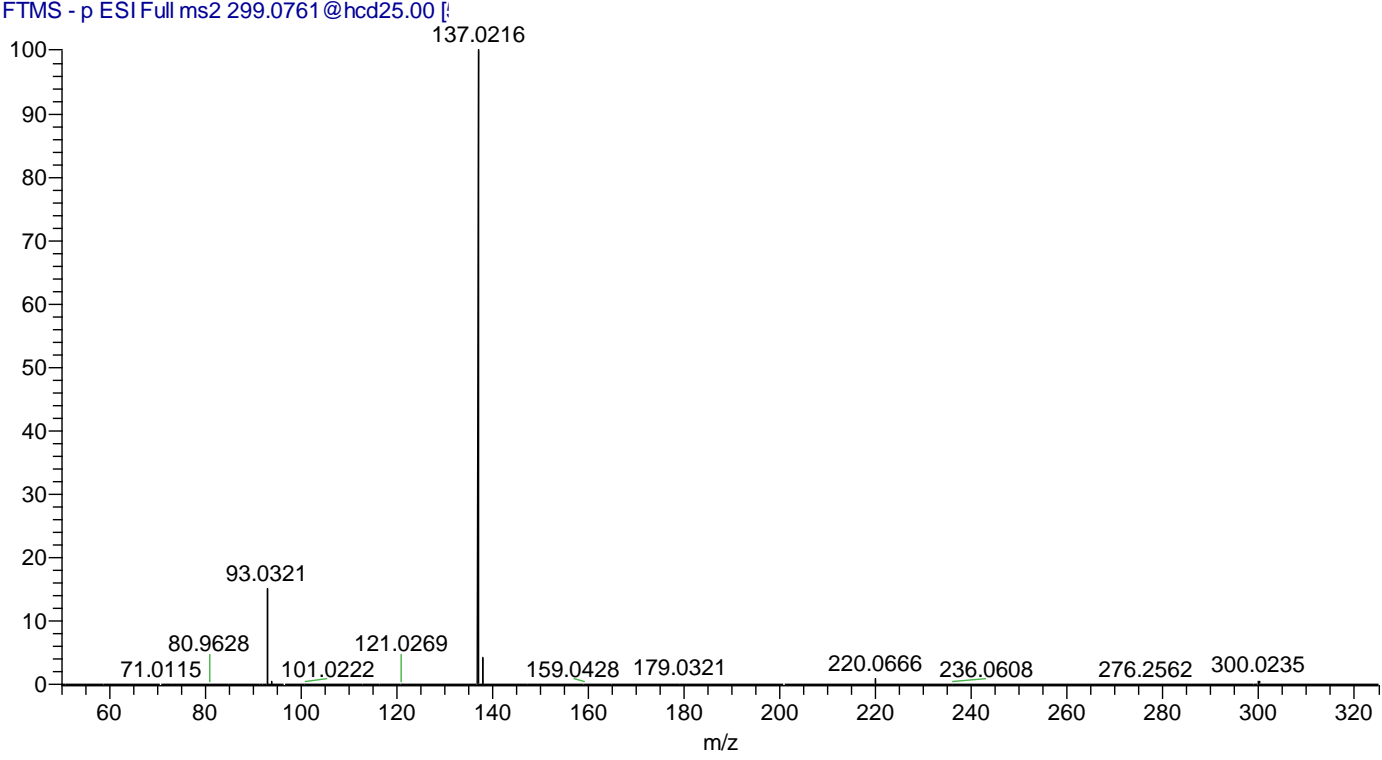

MS/MS in the negative mode of compound 22

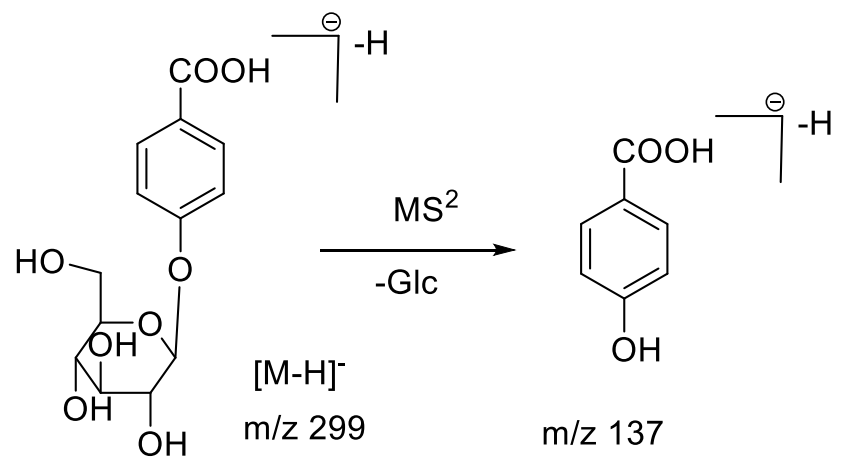

Proposed fragmentation of compound $\mathbf{2 2}$ 
FH97 \#109 RT: 0.32 AV: 1 NL: 1.26E9

T: FTMS - p ESI Full ms [100.0000-1000.0000]

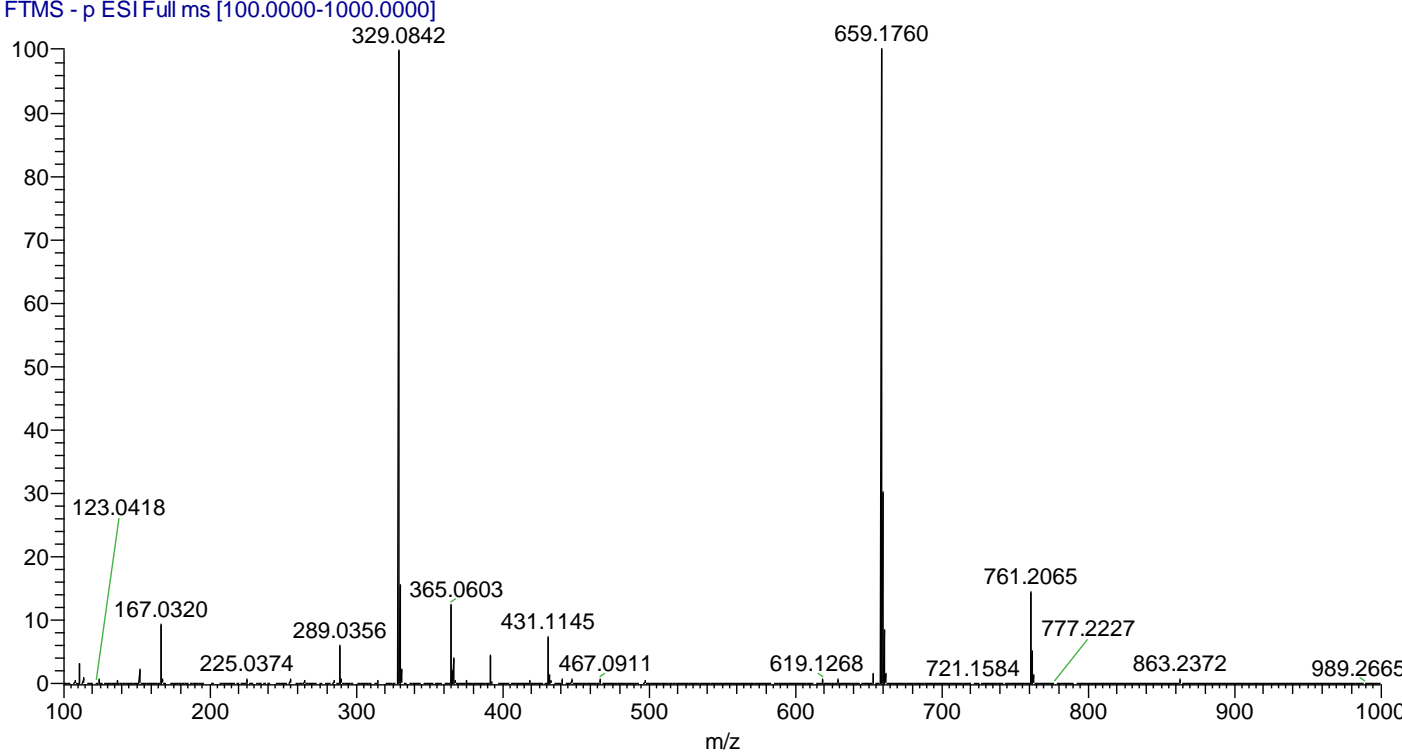

HRESIMS in the negative mode of compound $\mathbf{2 3}$

FH97 \#108 RT: 0.31 AV: 1 NL: $5.30 E 8$

T: FTMS - p ESI Full ms2 329.0867@hcd25.00 [!

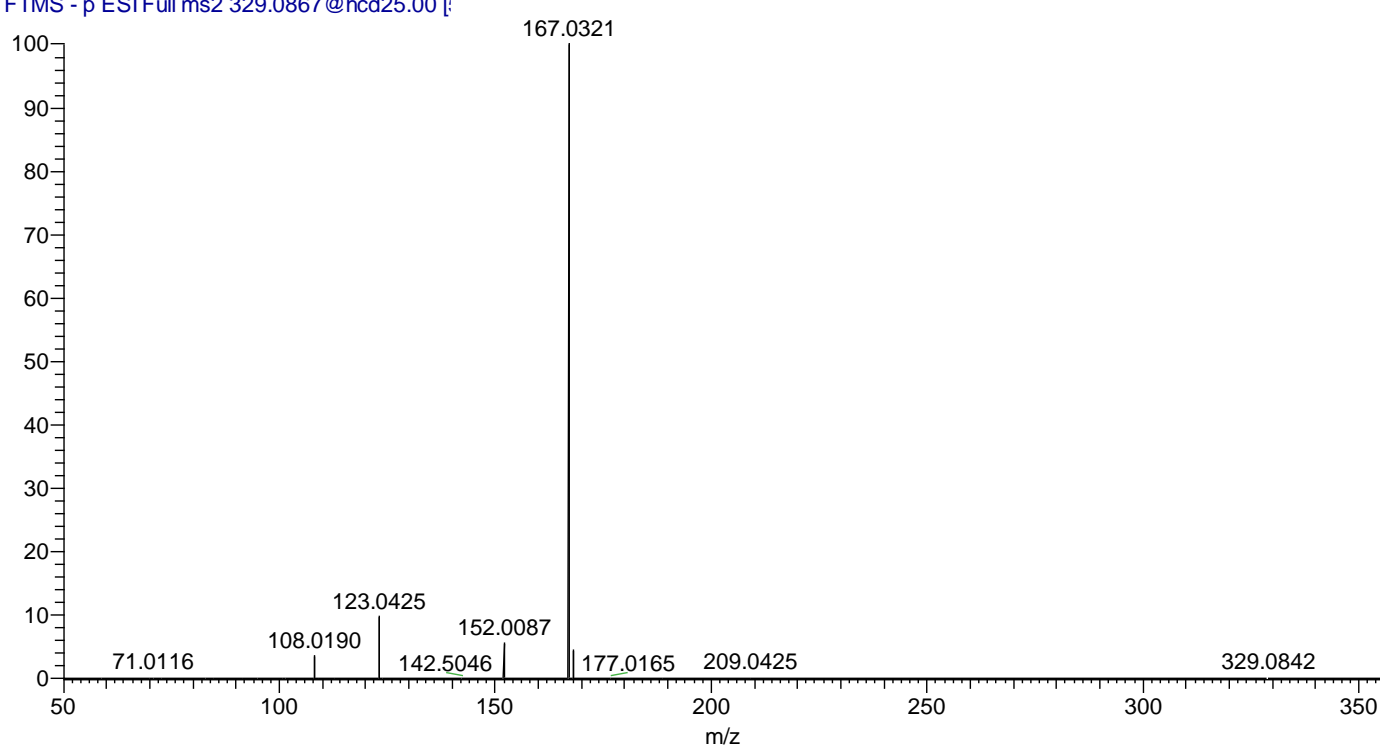

MS/MS in the negative mode of compound $\mathbf{2 3}$<smiles>COc1cc(C(=O)OC(C)C)ccc1O</smiles>

Proposed fragmentation of compound $\mathbf{2 3}$ 
FH38 \#121 RT: 0.35 AV: 1 NL: $1.28 E 9$

T: FTMS - p ESI Full ms [100.0000-1000.0000]

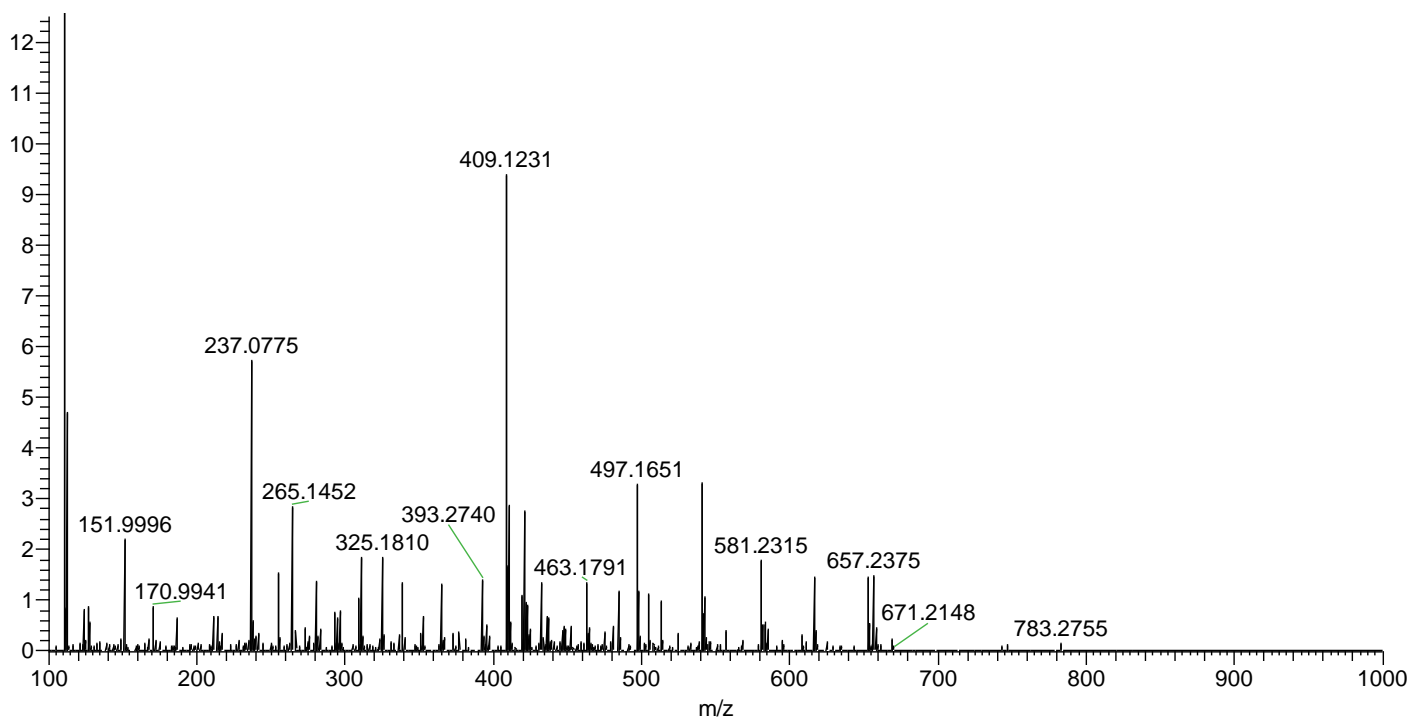

HRESIMS in the negative mode of compound $\mathbf{2 4}$

FH38 \#120 RT: 0.35 AV: 1 NL: $1.71 \mathrm{E} 6$

T: FTMS - p ESI Full ms2 373.1493@hcd25.00 [!

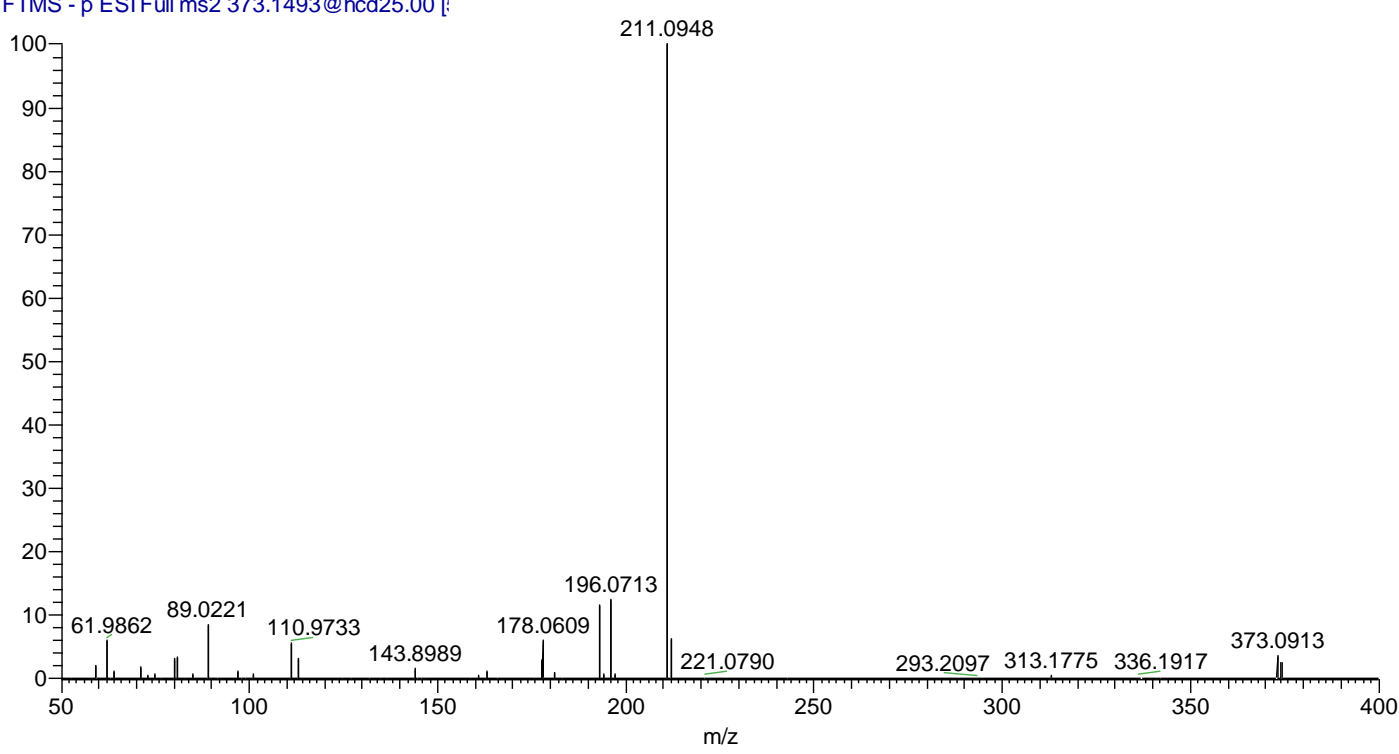

MS/MS in the negative mode of compound $\mathbf{2 4}$

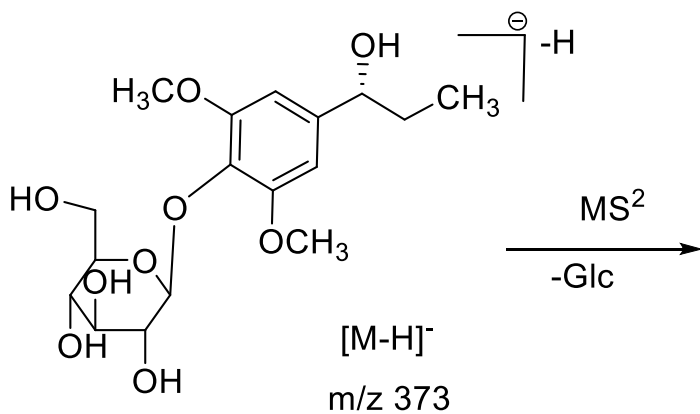<smiles>CC[C@H](O)c1cc(OC)c(O)c(OC)c1</smiles>

Proposed fragmentation of compound $\mathbf{2 4}$ 


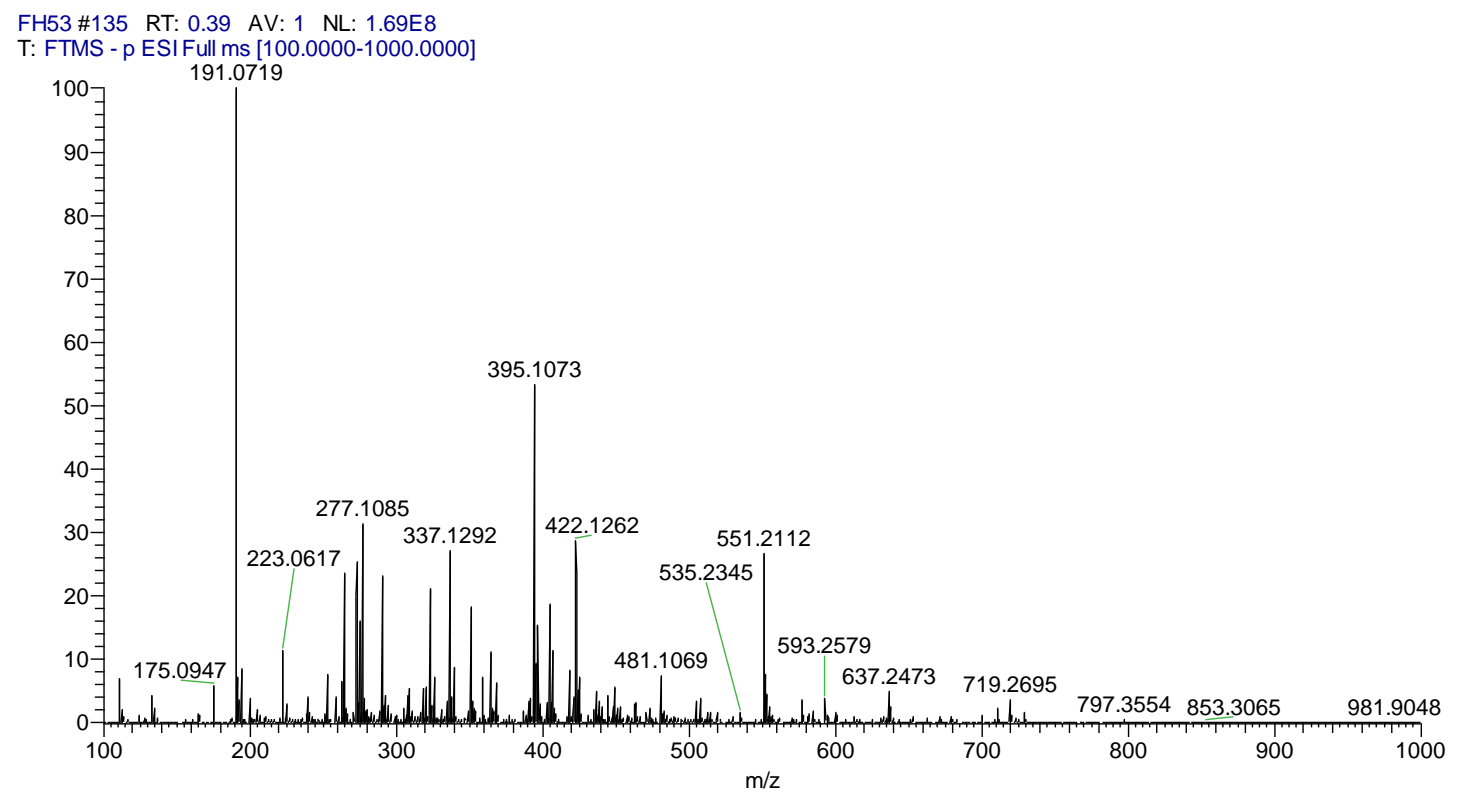

HRESIMS in the negative mode of compound $\mathbf{2 5}$

FH53 \#136 RT: 0.39 AV: 1 NL: 4.91E5

T: FTMS - p ESI Full ms2 359.1337@hcd25.00 [!

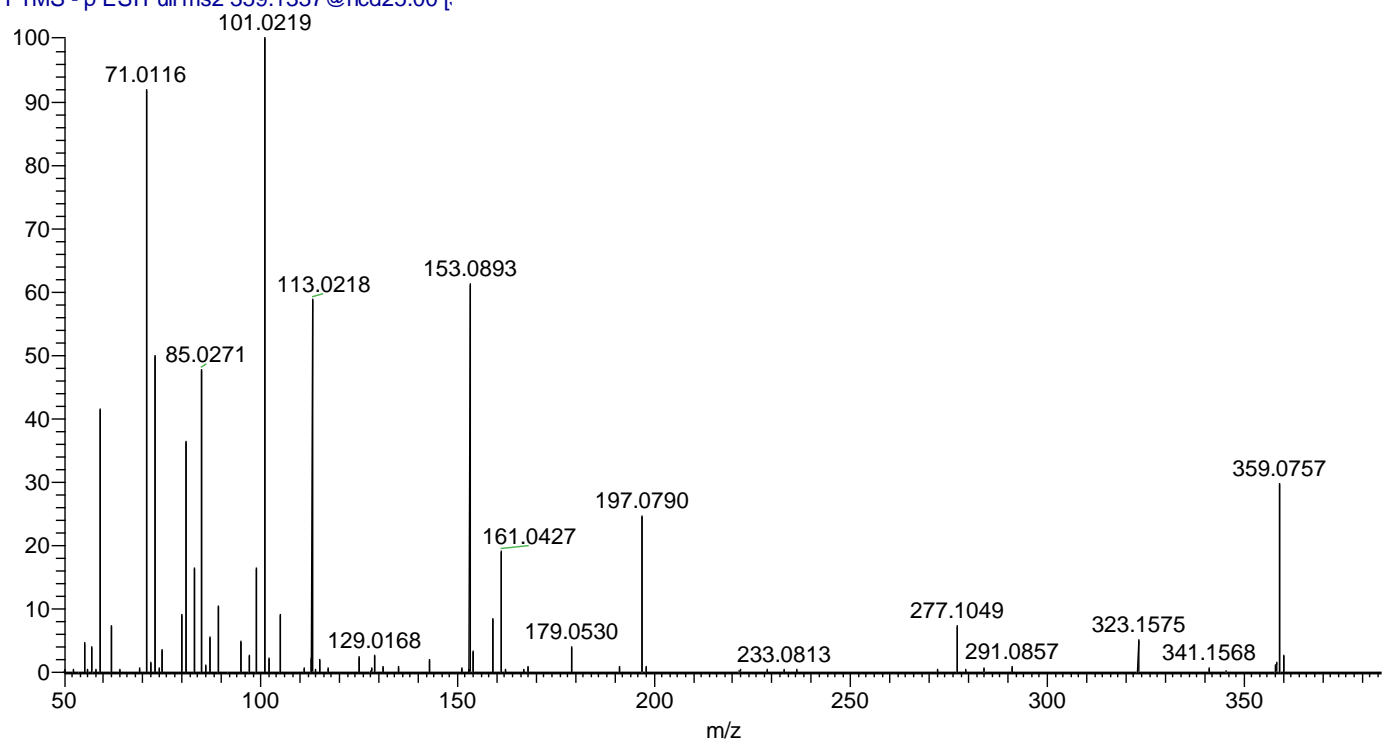

MS/MS in the negative mode of compound 25<smiles>COc1cc(COC2C(O)C(O)C(CO)OC2C(O)CO)cc(OC)c1OC</smiles>

Proposed fragmentation of compound $\mathbf{2 5}$ 


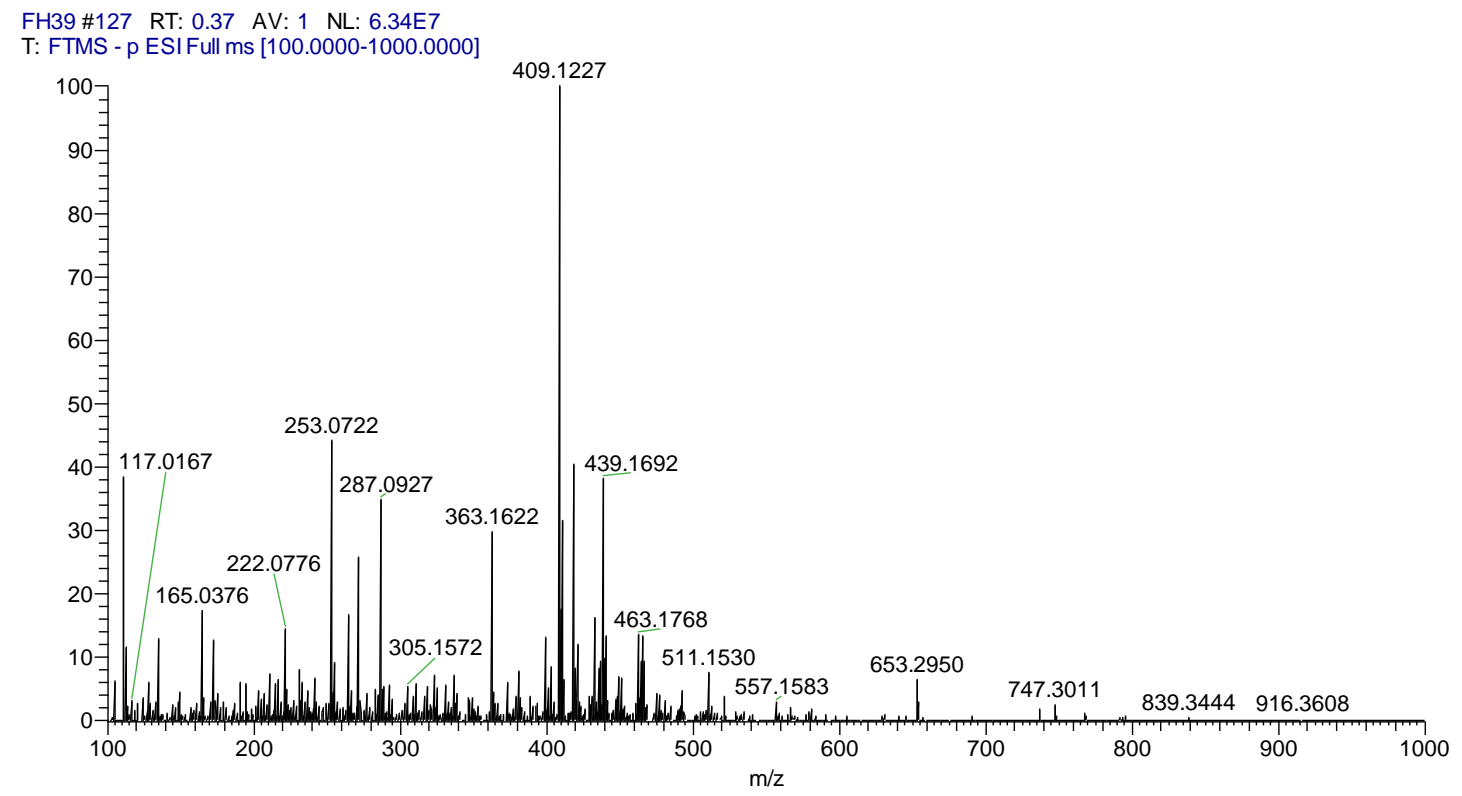

HRESIMS in the negative mode of compound $\mathbf{2 6}$

FH39 \#126 RT: 0.37 AV: 1 NL: 1.38E6

T: FTMS - p ESI Full ms2 373.1493@hcd25.00 [!

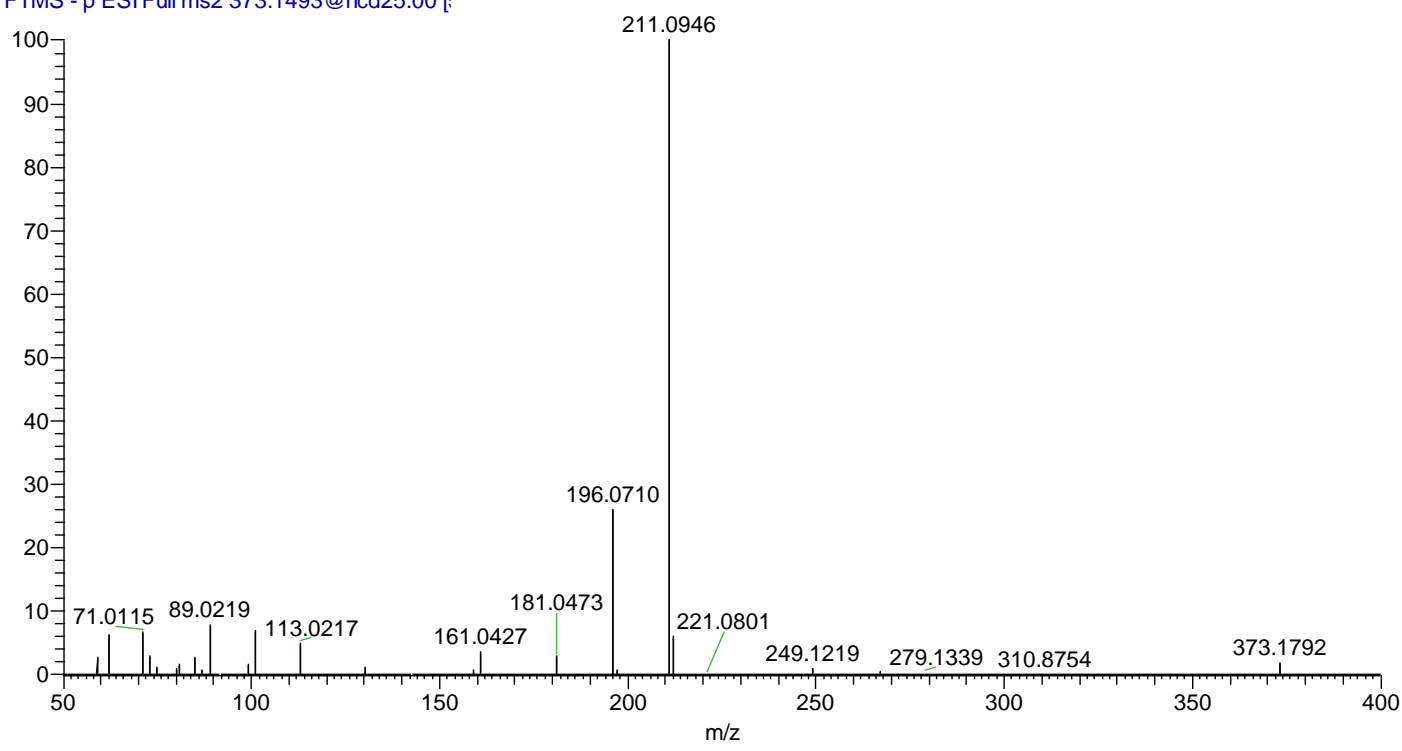

MS/MS in the negative mode of compound 26<smiles>COc1cc(CCCOC(OCCO)C(O)C(O)O)cc(OC)c1O</smiles><smiles>COc1cc(CCCO)cc(OC)c1O</smiles>

$\mathrm{m} / \mathrm{z} 211$

Proposed fragmentation of compound $\mathbf{2 6}$ 


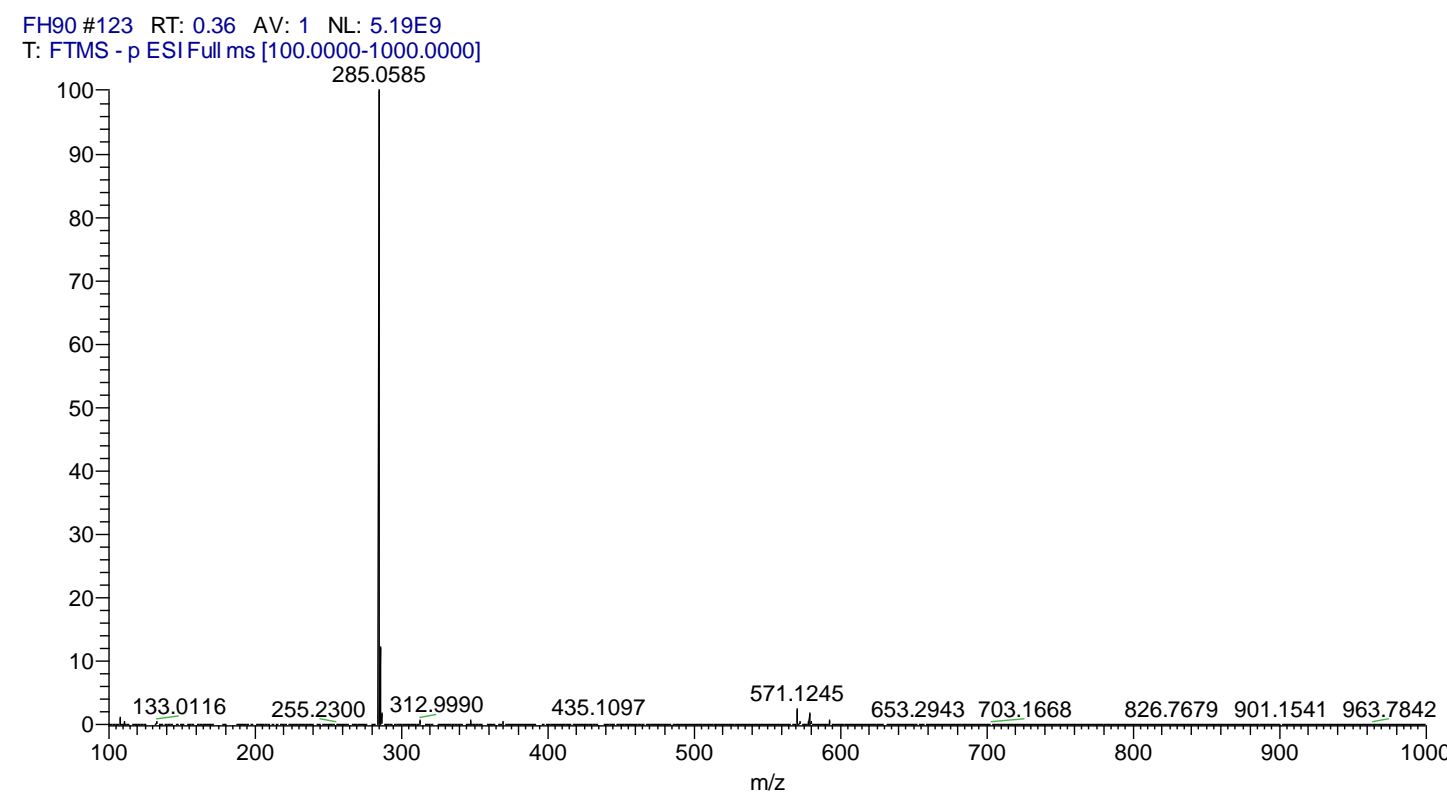

HRESIMS in the negative mode of compound 27

FH90 \#124 RT: 0.36 AV: 1 NL: 4.75E5

T: FTMS - p ESI Full ms2 315.1074@hcd25.00 [!

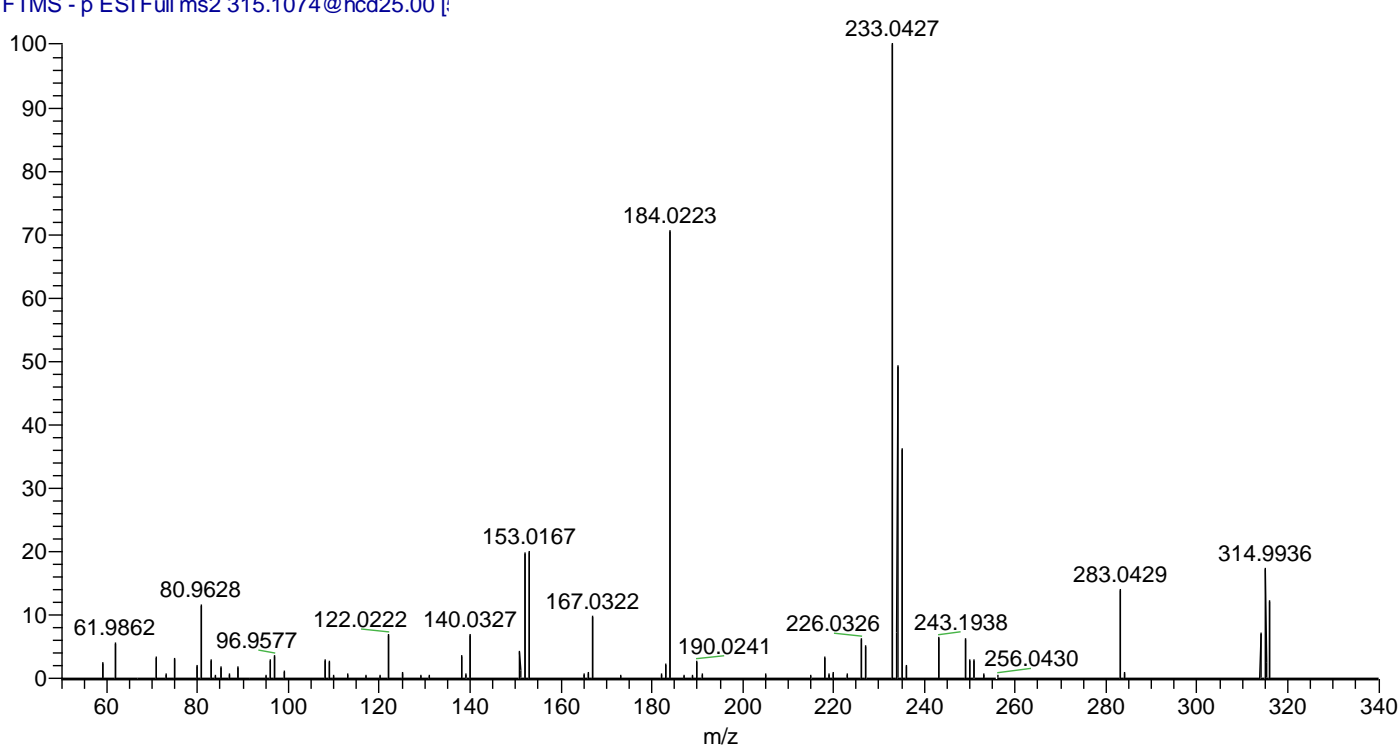

MS/MS in the negative mode of compound 27

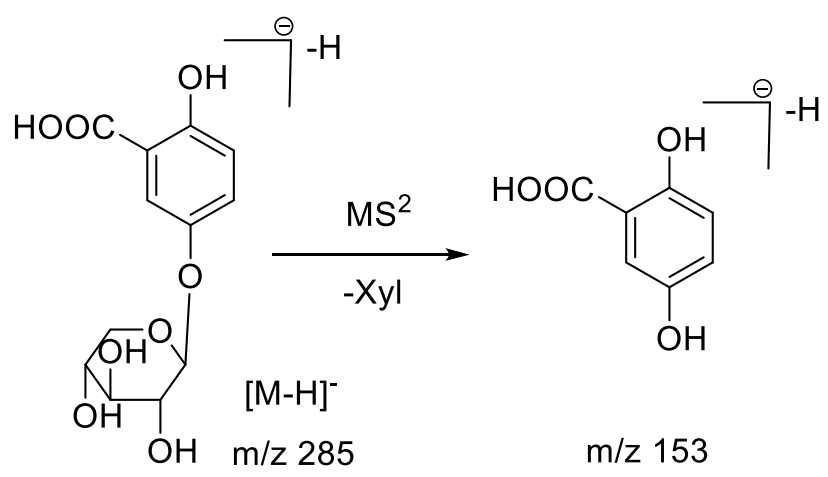

Proposed fragmentation of compound 27 

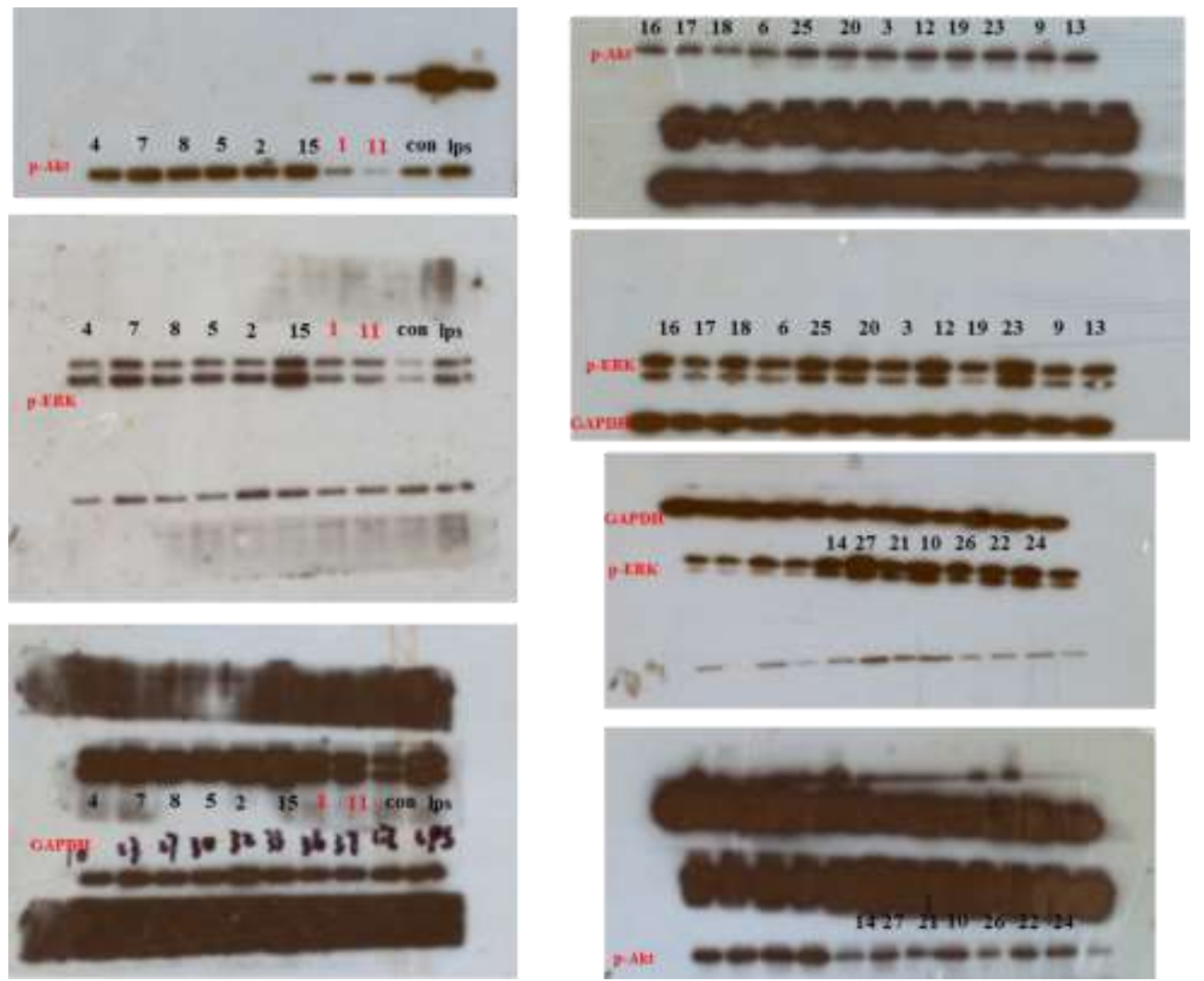

Figure S59. The origin Western blot picture of the p-Akt and p-ERK
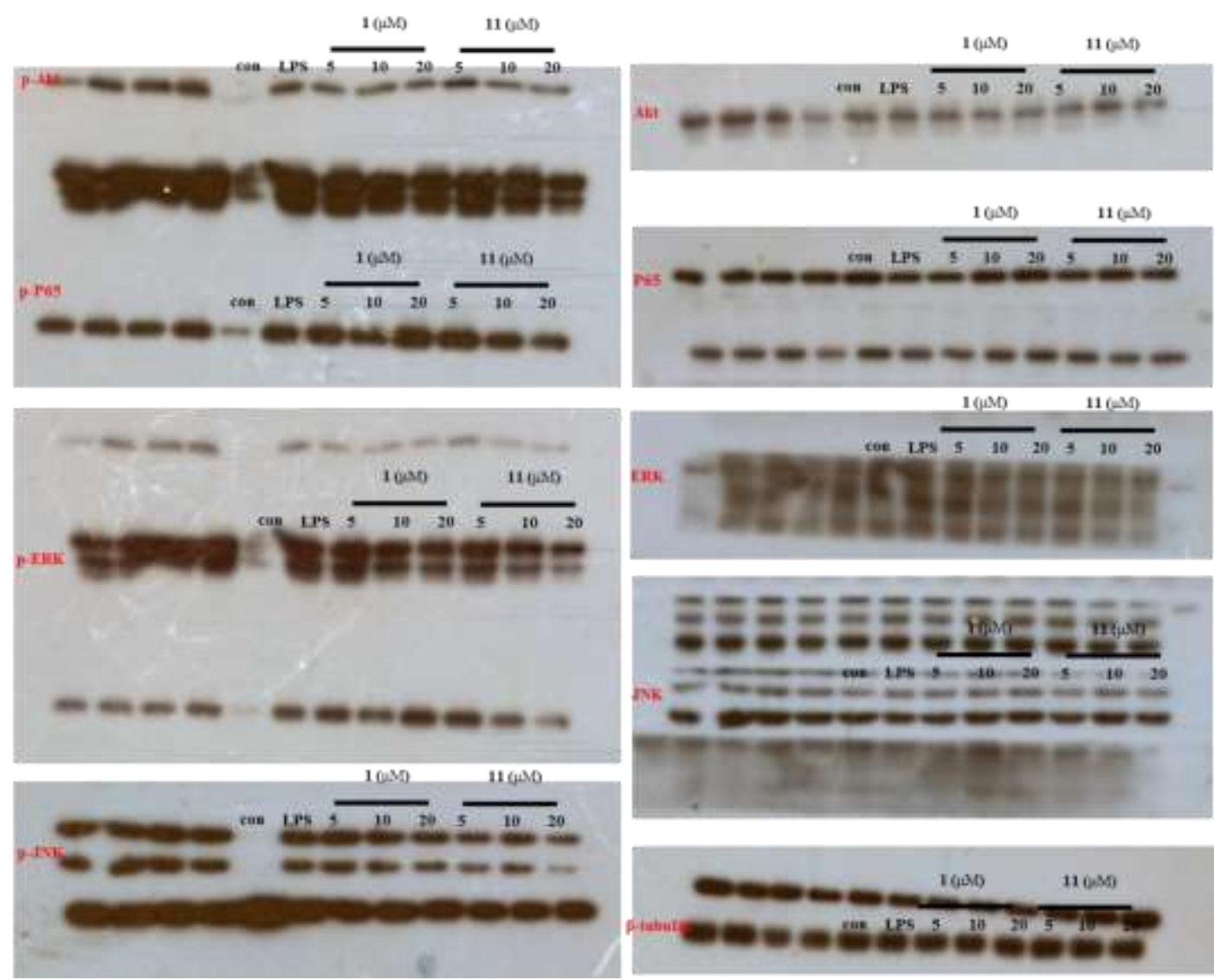

Figure S60. The origin Western blot picture of the p-p65, p-Akt, p-ERK and p-JNK 